\title{
3
}

\section{Nutritionally Enhanced Staple Food Crops}

Sangam L. Dwivedi, Kanwar L. Sahrawat, and Kedar N. Rai International Crops Research Institute for the Semi-Arid Tropics (ICRISAT)

Patancheru, 502324

Andhra Pradesh, India

Matthew W. Blair

Department of Plant Breeding

242 Emerson Hall

Cornell University

"Ithaca, NY 14853, USA

Meike S. Andersson and Wolfgang Pfeiffer

HarvestPlus.

c/o Centro Internacional de Agricultura Tropical (CIAT) Apartado Aéreo 6713, Cali, Colombia

\section{ABSTRACT}

Crop biofortification is a sustainable and cost-effective strategy to address malnutrition in developing countries. This review synthesizes the progress toward developing seed micronutrient-dense cereals and legumes cultivars by exploiting natural genetic variation using conventional breeding and/or transgenic technology, and discusses the associated issues to strengthen crop biofortification research and development. Some major QTL for seed iron and zinc, seed phosphorus, and seed phytate in common bean, rice, and wheat have been mapped. An iron reductase QTL associated with seed-fron QTL is found in 
common bean where the genes coding for candidate enzymes involved in phytic acid synthesis have also been mapped. Candidate genes for lpa cosegregate with mutant phenotypes identified in rice and soybean. The $G p c-B 1$ locus in wild emmer wheat accelerates senescence and increases nutrient remobilization from leaves to developing seeds, and another gene named TtNAM-B1 affecting these traits has been cloned. Seed iron-dense common bean and rice in Latin America; seed iron-dense common bean in eastern and southern Africa; seed iron-dense rice in the Philippines; and $\beta$-carotene-rich sweet potato in Latin America have been released for cultivation, with more nutritionally enhanced lines in pipeline for release, mostly in developing countries. Exceptionally large variations in $\beta$-carotene have been reported in temperate maize germplasm, which have been transferred into tropical maize hybrids, being evaluated prior to their release in Mexico and in some countries in Africa. The high $\beta$-carotene trait in 'Golden Rice 2' is being introgressed into several Asian rice cultivars.' At CIMMYT, molecular markers for $L y c E$ and $H y d B$ are fully implemented, have accelerated breeding by one season, and substantially enhance efficiency and effectiveness of high-provitamin A maize breeding. Marker-assisted selection has been successfully employed to transfer low phytate into improved soybean cultivars. Biofortified crops are being investigated for efficacy with human and animal systems. Transgenic rice containing AtNAS and $p_{V f e r r i t i n}$ have increased seed iron several folds, while transgenic maize containing phyA2 shows high phytase activity and reduced phytic acid compared to wild types. Maize transgenics containing five carotenoid genes show higher accumulation of $\beta$-carotene with no adverse effects on agronomic traits. A rigorous assessment is suggested to identify advantages of biofortified food for human health. Several issues, in addition to the above, related to biofortification, have been highlighted for furthering biofortification research in staple food crops. Biofortification has been included as core breeding activity in some countries in Latin America to ensure that newly developed crop cultivars meet nutritional needs of humans.

KEYWORDS: applied genomics; bioavailability; biosafety; $\beta$-carotene; crop biofortification; micronutrients

\section{ABBREVIATIONS}

I. INTRODUCTION

II. BIOMARKERS FOR ASSESSING NUTRITIONAL STATUS

III. MICRONUTRIENT BIOAVAILABLITY

A. Models and Assays to Access Nutrients Bioavailability and Absorption

1. Models and Assays

2. Nutrients Bioavailability, Absorption, and Metabolism

3. Iron and Zinc

4. $\beta$-Carotene

B. Factors Influencing Nutrients Bioavailability

1. Enhancers/Inhibitors 
2. Production Environment, Postharvest and Storage Conditions, and Food Processing and Preparation

3. Provitamin A ( $\beta$-Carotene) Conversion to Retinol (Vitamin A)

C. Efficacy of Biofortified Crops on Human Health

IV. PHENOTYPIC SCREENS

A. Methodology and Approaches to Screen for Seed Iron, Zinc, Phytate, and $\beta$-Carotene

B. Screening Under Optimal or Nutrient-Deficient Conditions

C. Plant Traits Associated with Increased Acquisition of Iron and Zinc

D. Iron and Zinc Uptake, Accumulation, and Translocation to Seed and Nonseed Parts

E. Nutrient Use Efficiency

V. MINING GERMPLASM COLLECTIONS FOR NATURALVARIATION FOR SEED IRON, ZINC, AND PHYTATE

A. Variation and/or Bioavailability of Seed Iron, Zinc, and Phytate

1. Seed Iron and Zinc Concentration

2. Seed Phytate Concentration

3. Seed Iron and Zinc Bioavailability

B. Distribution of Iron and Zinc in the Seed

VI. EXPLOITING NATURAL GENETIC VARIATION TO BREED FOR SEED MINERAL-DENSE CULTIVARS

A. Fixing the Biologically Attainable Target to Breed for Seed Mineral-Dense Crops :

B. Genotype $\times$ Environment Interaction and Relationships Between Seed Minerals and Agronomic Traits

C. Quantitative Trait Loci (QTL) Associated with Seed Iron, Zinc, and Phytate Concentrations

D. QTL Mapping, Cloning, and Introgression of $\beta$-Carotene into Adapted Germplasm

E. Developing Seed Iron- and Zinc-Dense Cultivars Using Conventional Breeding and Genomic Tools and Cultivars Adoption

1. Grain Minerals-Dense Cultivars and Hybrids

2. Adoption of Biofortified Cultivars by Farmers

F. Breeding Issues Associated with Selecting Seed Mineral-Dense Progenies

VII. ENHANCING SEED IRON, ZINC, AND $\beta$-CAROTENE USING TRANSGENE(S)

A. Transgenes for Nutritional Enhancement of Food Crops

1. Iron and Zinc

2. Phytate

3. $\beta$-Carotene

B. Consumer's Attitude to Genetically Modified Biofortified Crops

C. Nutritionally Enhanced Genetically Modified Crops and Biosafety Issues

VIII. OUTLOOK

IX. ACKNOWLEDGMENTS

LITERATURE CITED 


\section{ABBREVIATIONS}

AAS

AGP

$\beta$-carotene

BMI

CB

cDNAs

CGIAR

CIAT

CIMMYT

CM

CRP

crtRB1

DALYS

DMA

DNA

$\mathrm{E}$

EDX

epi-HDMA

epi-HMA

$\mathrm{Fe}^{2+}$

$\mathrm{Fe}^{3+}$

FISH

FIGS

G

GEI

GI

GISH

GM

Gpx1

$\mathrm{Hb}$

HI

HPLC

ICAR

Atomic absorption spectrophotometer

$\alpha_{1}$-Acid glyco protein

Beta carotene

Body mass index

Conventional bean

Complementary deoxyribonucleic acids

Consultative Group on International Agricultural

Research

Centro Internacional de Agricultura Tropical

International Maize and Wheat Improvement Center

Conventional maize

C-reactive protein

$\beta$-Carotene hydroxylase 1

Disability-adjusted life years

2 '-Deoxymugineic acid

Deoxyribonucleic acid

Environment

Energy dispersive X-ray microanalysis

3-Epihydroxy-2'-deoxymugineic acid

3-Epihydroxymugineic acid

Ferrous iron

Ferric iron

Fluorescent in situ hybridization

Focused Identification of Germplasm Selection

Genotype

' Genotype environment interaction.

Gastrointestinal

Genomic in situ hybridization

Genetically modified

Glutathione peroxidase-1

Hemoglobin

Harvest index

High performance liquid chromatography

Indian Council of Agricultural Research

ICARDA International Center for Agricultural Research in the Dry Areas

ICP Inductively coupled plasma

ICRISAT International Crops Research Institute for the Semi-Arid Tropics

IITA 


\begin{tabular}{ll} 
IRA & Iron reductase activity \\
IRRI & International Rice Research Institute \\
LG & Linkage group \\
LCYE & Lycopene e-cyclase \\
LMWOA & Low molecular weight organic acids \\
lp & Low phytate \\
MA & Mugineic acid \\
MABC & Marker-assisted backcrossing \\
MAS & Marker-assisted selection \\
MGT & Multigene transfer \\
mRNA & Messenger ribonucleic acid \\
MRP & Multidrug resistance-associated protein \\
nano-SIMS & Nano-secondary ion mass spectrometry \\
NARs & National agricultural research systems \\
NEB & Nutritionally enhanced bean \\
NEM & Nutritionally enhanced maize \\
NIRS & Near infrared reflectance spectroscopy \\
OFSP & Orange-fleshed sweet potato \\
PFDS & Public food distribution system \\
Pi & Inorganic phosphorus \\
PIXE & Proton-induced X-ray emission \\
PS & Phytosiderophore \\
QPM & Quality protein maize \\
QTL & Quantitative trait loci \\
STEM & Scanning and transmission electron microscopy \\
VPKAS & Vivekananda Parvatiya Krishi Anusandhan Sansthan \\
WT & Wild type \\
$\mu$-XRF & Micro X-ray fluorescence \\
\hline &
\end{tabular}

\section{INTRODUCTION}

The world's population is projected to grow by $34 \%$ from the current 6.8 billion to 9.1 billion in 2050 , with nearly all of this increase in developing countries. Meanwhile global demand for food during the same period is projected to be $70 \%$ higher than today, involving an additional annual consumption of nearly 1 billion tonnes of cereals for food and feed and 200 million tonnes of meat (World Summit on Food Security 2009, here cited as WSFS 2009). The accumulated evidence reveals that not only crop yields decline at temperatures much above $30^{\circ} \mathrm{C}$ (Schlenker and Roberts 2009) but also the produce from such crops is less nutritious (Qaderi and Reid 2009). Climate models predict that 
warmer temperatures and increases in the frequency and duration of drought during the 21st century will have net negative effects on agricultural productivity. It is predicted that $\mathrm{CO}_{2}$ level in the atmosphere is expected to rise due to climate change and studies in wheat, barley, rice, and rapeseed have shown significant declines in total protein and essential amino acids, as well as a range of macro- and micronutrients (Högy and Fangmeier 2008; Taub et al. 2008; Högy et al. 2009, 2010; Erbs et al. 2010). Furthermore, the negative impacts of climate change on soil fertility and mineral nutrition of crops will far exceed beneficial effects, with serious consequences to food security in developing countries (St. Clair and Lynch 2010). To feed $\sim 9$ billion people by the middle of 21st century, the production of high-quality food must increase with reduced inputs. Plant breeders need to focus on traits with the greatest potential including resistance/tolerance to biotic and abiotic stresses to increase yield. New technologies can help accelerate breeding in such a way that it increases the available genetic diversity to improve food and nutritional security (Fedoroff et al. 2010; Tester and Langridge 2010).

Micronutrient malnutrition arising from Zn and Fe deficiencies alone affect over 3 billion people around the world (http://www.unscn.org). Nearly 500,000 children $<5$ years of age die annually because of $\mathrm{Zn}$ and Fe deficiencies (Black et al. 2008). Furthermore, micronutrient malnutrition is accompanied by serious physical incapacity, mental impairment, decreased health, and parasitic diseases. The number of chronically undernourished people has risen from 842 million at the beginning of the 1990s to over 1 billion in 2009 (World Summit on Food Security 2009; Barrett 2010). Fighting malnutrition is an integral component of three of the eight millennium development goals, that is; eradication of extreme poverty and hunger, reduction of child mortality, and improvement of maternal health (http://www.UNESCAP.ORG/ publication/survey2002/suro2-ii.pdf), with World Summit on Food Security endorsing to halt immediately the increase in and to significantly reduce the number of people suffering from hunger, malnutrition, and food insecurity, and reinforce efforts to meet by 2015 the targets of the millennium development goals (Sachs and McArthur 2005).

Widespread micronutrient malnutrition results in an enormous negative socio-economic impact at the individual, community, and national levels (Darnton-Hill et al. 2005; Stein 2010). Among the 26 major risk factors of the global burden of disease estimates, iron deficiency ranks 9th, zinc deficiency 11th, and vitamin A deficiency 13th (Ezzati et al. 2002). Disability-adjusted life years (DALYs) are 
used to establish the burden of a disease by measuring the health loss through mortality and morbidity in a single index (Murray and Lopez 1996). In India, over 9 million DALYs are lost annually due to iron; zinc, and vitamin A deficiencies, with iron deficiency alone contributing to 4 million DALYs lost (Qaim et al. 2007). The disease burden associated with iron deficiency in India could be reduced by $19 \%-58 \%$ by crop biofortification (Stein et al. 2008). Likewise, Stein et al. (2007) reports annually 2.8 million DALYs lost due to zinc deficiency in India. Zinc biofortified rice and wheat may reduce this burden by $20 \%-51 \%$ and save $0.6-1.4$ million DALYs each year. The costs for saving one DALY through crop biofortification amount to US\$ 0.73-7.31, which is very cost-effective and lower than that of most other micronutrient interventions (Stein 2006; Ma et al. 2007). More recently, Meenakshi et al. (2010) concluded that overall, biofortification can make a significant impact on reducing the burden of micronutrient deficiencies in the developing world in a highly cost-effective manner; however, the impacts differ depending on the combination of crop, micronutrient and country, and the major reasons underlying these differences are identified to inform policy.

In the past, food fortification, diet diversification, and nutrient supplementation have been most frequently used as public health interventions to reduce micronutrient-induced morbidity and mortality worldwide (Suharno et al. 1993; Haider et al. 2003; Brown et al. 2007; Wienecke and Gruenwald 2007; Casey et al. 2010; Eneroth et al. 2010). However, these approaches have had only limited success and could not by themselves attain sufficiently millennium development goals mainly because such interventions require infrastructure, continuous flow of resources, purchasing power, or access to markets and health care systems to their success, often not available to people living in remote areas. (Underwood 1999; Imhoff-Kunsch et al. 2007; Ssemakula and Pfeiffer 2011).

The rural-based diets are predominantly composed of cereals and legumes, with limited access to fruits and vegetables that are regarded as rich sources of minerals and vitamins. The consumption of fruits and vegetables along with staple foods facilitates more bioaccessible micronutrients that otherwise would be inaccessible due to the presence of antinutrients, such as phytates and tannins (Ballot et al. 1987; Ali and Tsou 1997; Jansen van Rensburg et al. 2004; Flyman and Afolayan 2006; Baruah and Borah 2009). Integrating micronutrient-rich foods such as legumes, vegetables, and fruits into diets is the most practical and sustainable way to alleviate micronutrient deficiency. 
An alternative (or complement) to the above approaches is to use plant breeding to naturally fortify commonly consumed staple crops with micronutrients, a process known as genetic biofortification (Bouis 2003). Biofortification has the potential to help alleviate the suffering, death, disability, and failure to achieve full human potential that result from micronutrient deficiency-related diseases (Haas et. al. 2005; Campos-Bowers and Wittenmyer 2007; Rosado et al. 2009; Arsenault et al. 2010; Bouis and Islam 2011). In summary, therefore, biofortification refers to the development of micronutrient-dense staple crops using traditional breeding practices or biotechnology (Bouis 2003). Crop biofortification offers the unique opportunity to create international public goods (IPGs) by exploiting natural genetic variation to develop seed mineral-dense crops. A 10-year Consultative Group on International Agriculțral Research (CGIAR) Challenge Program on "Biofortified Crops for Improved Human Nutrition" was initiated in 2004 with support from the Bill and Melinda Gates Foundation, the World Bank, and USAID to improve the health of poor people by breeding staple food crops that are rich in iron, zinc, and vitamin A ( $\beta$-carotene), with full-time breeding programs initiated on rice, wheat, maize, cassava, sweet potato, and common beans consumed by the many of the world's poor (www.harvestplus.org). Prebreeding feasibility studies have been performed on banana, barley, cowpea, groundnut (peanut), lentil, pearl millet, pigeon pea, plantains, potatoes, sorghum, and yams. This program is a multinational, multisectoral, multidisciplinary, multicrop, multinutrient, and multipartnered undertaking. (Pfeiffer and McClafferty 2007; Bouis and Islam 2011).

In agronomic terms, the mineral-dense seeds of biofortified cultivars often produce more viable and vigorous seedlings and such plants are more efficient in mineral uptake thus improving disease resistance and some growth characteristics (Rengel and Graham 1995; Graham and Welch 1996). Welch and Graham (2005) report that the improved seedling vigor is associated with the production of more and longer roots under micronutrient-deficient conditions, allowing seedlings to scavenge more soil volume for micronutrients and water early in growth, an advantage that can lead to improved yields compared to seeds with low micronutrient stores grown on the same soils. Additionally, greater stress tolerance has been noticed in seedlings grown from micronutrient-dense seeds resulting in higher agricultural production (Welch 1986; Cakmak 2008). Agronomic biofortification is not the subject of this review but includes cropping systems diversification, fertilizer enrichment, seed priming, or use of soil microorganisms such as mycorrhizal fungi to improve soil rhizosphere environments for 
better acquisition of immobile mineral elements from the root zones (He and Nara 2007; Cakmak 2008; Cavagnaro 2008; Khoshgoftarmanesh et al. 2010).

A final issue of utmost importance is adoption of biofortified cultivars. The farmer's perception of adoptability of a new cultivar must be taken into account and is such that new cultivar should show yield superiority and/or tolerance to stresses and produce more than the existing cultivars with respect to seed yield and other characteristics such as taste, size, color, and flavor. For biofortified crops, there should be assured market and the produce must earn farmers more income if the cultivar is to be widely adopted and therefore the nutritional benefit is to be widespread. Public investments in research to create biofortified crops have increased in recent times because of the growing awareness about the importance of micronutrient deficiencies and associated adverse health effects. Consumer acceptance of the biofortified crops could be an issue if the new intervention changes the appearance or taste of the food crops (Wolson 2007); however, this may not be the case for the crops biofortified with so-called "invisible traits" such as iron or zinc. Crops fortified with $\beta$-carotene exhibit a deep yellow to orange color as seen in the case of golden rice, orange-fleshed sweet potato (OFSP); and yellow cassava (Pray et al. 2007; Ramaswami 2007), which may limit their acceptance as food. The biosafety issues of nutritionally enhanced transgenic crops, whether this is for iron and zinc or for provitamin $\mathrm{A}$, is another issue that should be adequately addressed to allay consumers concern about the safety of the genetically modified (GM) crops.

This review focuses on the progress realized toward developing seed nutrient-dense ( $\mathrm{Fe}, \mathrm{Zn}$, and $\beta$-carotene) cultivars of cereals and legumes by exploiting natural genetic variation using conventional breeding and/or transgenic technology. Associated with this we also discuss other critical issues such as: (1) the need to develop rapid, cost-effective, and accurate phenotypic screening tools/techniques; (2) traits associated with increased nutrient use efficiency, storage, and reallocation of nutrients; (3) models and biomarkers for assessing bioavailability and diagnosing micronutrient deficiency; (4) factors associated with bioavailability of micronutrients; (5) micronutrient distribution in seeds; (6) genotype (G) by environment interaction and the nature of associations (and trade-offs) between micronutrients and agronomic traits; (7) markers and quantitative trait loci (QTL) associated with increased micronutrients; and (8) biosafety issues associated with the use of genetically modified crops for biofortification. 


\section{BIOMARKERS FOR ASSESSING NUTRITIONAL STATUS}

Biomarker is a characteristic that is objectively measured and evaluated as an indicator of normal biologic and pathogenic processes, or pharmacologic responses to a therapeutic intervention (http://www. everythingbio.com/glos/definition.phf?ID=3716). The use of a biomarker that reflects the changes in the micronutrient status can indeed facilitate the understanding of the relationships between dietary micronutrient intake and the status, and those between micronutrient status and health. Anthropometry (measuring weights and heights and other body measurements) and biochemical tests (mostly blood, sometimes urine) are the two most often used methods to assess the nutritional status of the populations or individuals. Anthropometry is the universally applicable, inexpensive, and noninvasive technique for the assessment of growth and nutrition. The basic anthropometric measurements include the body mass index $\left(\mathrm{BMI}=\right.$ weight/height $\left.{ }^{-2}\right)$ and the waist circumference (either alone or expressed against hip circumference).

The BMI is the most useful parameter as a direct measure of over- or underweight as classified by the World Health Organization (WHO): BMI < 18.50 indicates underweight, 18.5-24.99 normal range, 25.0029.99 overweight, $>30.00$ obese, and $>40.00$ very severely obese. The waist circumference correlates quite closely with the BMI. The weightfor-age is the primary index, based on the U.S. Center for Disease Control growth reference, for measuring children's growth. In the weight-for-age graphs, the average is the 50th percentile (i.e., median) of the reference sample: below $80 \%$ of the median, a child is "underweight", while a child under $60 \%$ of the median is seriously underweight and has marasmus (http://www.oup.com/uk/orc/bin/ 9780199290970/mann_ch29f.pdf).

Biochemical methods are the essential part of the nutritional assessment. A number of biomarkers of iron status have been developed (Mei et al. 2005; Beard et al. 2006, 2007; Yang et al. 2008; Ayoya et al. 2010; Thurnham et al. 2010). Of these, the hemoglobin (Hb) concentration in the blood is commonly used to estimate the prevalence of iron deficiency. However, the $\mathrm{Hb}$ concentration is affected by factors besides the iron status, such as malaria, other systemic infections, hemoglobinopathies, and other micronutrient deficiencies (Yang et al. 2008). Diagnosing the iron deficiency among infants is a challenge, which may require additional measurements, besides $\mathrm{Hb}$, such as measurement of ferritin, soluble transferring receptor, and the protoporphyrin levels (Beard et al. 2007). Most recent studies revealed that either plasma ferritin concentration (Yang et al. 2008) or the measurement of both 
serum $\alpha_{1}$-acid glycoprotein (AGP) and serum C-reactive protein (CRP) (Ayoya et al. 2010) may provide better indication of the iron deficiency. However, it should be noted that the ferritin increases with infection; hence, the risk of underestimating the iron deficiency. Thurnham et al. (2010) therefore suggest measuring both acute-phase proteins (APP) and CRP to estimate the full effect of inflammation that can be used to correct ferritin concentrations. Lowe et al. (2009) assessed the usefulness of a number of potential biomarkers of the zinc status to conclude that plasma, urinary, and hair zinc in healthy individuals are the reliable biomarkers of zinc status in humans. Wessells et al. (2010) also confirmed the usefulness of measuring plasma $\mathrm{Zn}$ concentration to monitor compliance with, and possibly effectiveness of, Zn supplementation programs. The plasma Zn concentration in their study increases within 2-5 days of starting each of the doses of $\mathrm{Zn}$ supplements (10 and $20 \mathrm{mg} \mathrm{d}^{-1} \mathrm{Zn}$ ), remains elevated during the period of supplementation, and declines to baseline concentrations within 14 days of discontinuing supplementation. For the vitamin A deficiency, plasma $\beta$-carotene, which indicates reduced intake and plasma retinol that indicate impaired function or cell depletion have been suggested for an early diagnosis of the vitamin A deficiency (http:/www.oup.com/uk/orc/ bin/9780199290970/mann_ch29f.pdf).

Blood messenger ribonucleic acid (mRNA) biomarkers for individualized disease prediction and diagnosis are an exciting area in medicine, which offer early, and more accurate prediction and diagnosis of disease and disease progression, and thus the ability to identify individuals at risk. The mRNA biomarkers in nutrition have potential application to diagnose individuals/population suffering from nutritional deficiencies. For example, the changes in white cell metallothionein mRNA were found to correlate with the changes in zinc intake in human subjects (Cao and Cousins 2000), while the microarray analysis found decreased levels of mRNA for a zinc influx transporter in women supplemented with zinc (Andree et al. 2004). More recently, Sunde (2010) demonstrated that selenoprotein- $H$ and selenoprotein-W as well as glutathione peroxidase-1 (Gpx1) mRNA are highly down regulated in the selenium (Se) deficiency in rat liver, and the minimum dietary Se requirement based on these biomarkers is $0.06-0.07 \mu \mathrm{g} \mathrm{Se}^{-1}$, which is similar to those determined by using conventional biomarkers. Clearly, more research is needed to develop mRNA biomarker panels for all nutrients that will discriminate between deficient, marginal, adequate, and supernutritional individuals and populations, and differentiate between individuals who could benefit or be adversely affected by nutrient supplementation (Sunde 2010). 
In conclusion anthropometry and biochemical tests have been proposed to diagnose micronutrient malnutrition. Hb concentration in the blood is the most commonly used method to assess iron deficiency; however, several factors including iron status and inflammation in the body have potential to impact $\mathrm{Hb}$ concentration. It is therefore recommended that either plasma ferritin concentration or the measurement of both serum AGP and CRP may provide better indication of the iron deficiency. The zinc concentration in blood plasma, urine, or hair is reliable biomarker, while the plasma $\beta$-carotene and plasma retinol is used to diagnose vitamin A deficiency. Use of mRNA as biomarker is an emerging area and needs further investigation to develop mRNA biomarker panels for assessing the nutritional status of populations or individuals.

\section{MICRONUTRIENT BIOAVAILABILITY}

\section{A. Models and Assays to Access Nutrients Bioavailability and Absorption}

1. Models and Assays. Both in vitro and in vivo techniques have been used to assess bioavailability of minerals and vitamins, with both having their own strength and drawbacks. The in vitro technique is designed to mimic the human digestive system, particularly conditions in the stomach and small intestine. One well-known model uses cultured human Caco-2 cells, to evaluate the digestibility and bioaccessibility of nutrients. Caco- 2 cells are a human intestinal cell line originally derived from a colon adenocarcinoma to use as surrogate for enterocytes of the small intestine, which researchers bave used to assess the nutrients metabolism, transport, and absorption of various nutrients (Alvarez-Hernandez et al. 1991; Glahn et al. 1996; 1998a,b; Yun et al. 2004). The Caco-2 cell model is rapid, cost effective, and compares well with human studies $(r=>0.90)$ over a range of iron bioavailability for a known promoter or inhibitor (Au and Reddy 2000; Yun et al. 2004). The Caco-2 cell culture model has been used to compare iron bioavailability in common bean, maize, and rice to select those with high iron bioavailability (see Section III.A.2). Similar studies using in vivo models with laboratory animals are likely to cost many times a Caco-2 cell study and are often questioned given the validity of extrapolating from animals to humans (Reddy and Cook 1991). This method (Caco-2 cell culture) also allows researchers to study 
interactions between minerals such as $\mathrm{Fe}$ and enhancer or inhibitors affecting mineral bioavailability in food (Monsen et al. 1978; Yeung et al. 2003). Another advantage of Caco-2 cell model is that bioavailability can be determined even when composition data of a given food is either inaccurate or not available.

Thus, the Caco-2 cell culture model represents a useful tool for initial screening and should be complemented with in vivo studies that will remain as the criterion standard for bioaccessibility of nutrients and bioactive compounds (Fernández-García et al. 2009). Other in vitro methods include solubility and dialyzability that are not useful predictors of iron absorption in comparison to Caco-2 cell model, which provide the most useful in vitro experimental approach for studying iron availability from food digests to predict about the iron bioavailability in vivo. However, further developments are required to optimize and standardize methodologies between different laboratories, including cell type and passage number, cell culture conditions, use of dialysis membranes for food digests that contain ferritin and other large-molecular weight iron complexes, time of exposure to food digest, harvesting of cells, and the use of reference standards so that direct inter-laboratory comparisons can be made between different food substrates (Fairweather-Tait et al. 2005, 2007).

An in vivo method requires various types of test animals to access bioavailability of nutrients and nutrient precursors, nutrient $\times$ nutrient interactions, nutrient tolerances, and toxicities (Baker 2008). The two strategies in in vivo method include balance studies and tissue concentration, which allow determination of the absorbed amount of nutrients, bioactive compounds, or their metabolites. Balance studies determine the difference between the fed and excreted amounts of the nutrient or bioactive compound. The tissue concentration consists of monitoring the increase in plasma/serum concentration of the nutrient or bioactive compound. These approaches have been applied either with experimental animal or human subjects to determine absorption of carbohydrates, minerals, vitamins, phytochemicals, and others (Fernández-García et al. 2009).

The most frequently used animals for nutrition research are chicken, mouse, rat, gerbil, preruminant calf, ferret, nonhuman primate, pig or piglets, hamster, and dog (Lee et al. 1999), which have been valuable in advancing our knowledge of nutrition, with many exhibiting welldocumented differences versus humans in how they use, metabolize, and excrete nutrients (Lee et al. 1999; Baker 2008). However, because of species differences it is important to choose the right animal model for 
prediction of what might happen in humans. Other potential factors influencing the choice of animal model include the availability of facilities and cost of the experiments to be performed. Choosing the most appropriate model for a study requires careful consideration. No one animal model completely mimics human absorption and metabolism of nutrients; thus, the best model must be chosen with consideration of the specific application being studied, characteristics of the model, and the available resources and facilities.

\section{Nutrients Bioavailability, Absorption, and Metabolism. The key to} nutritional efficiency is bioavailability. It refers to the fraction of the nutrients or bioactive compounds available for use in physiologic function or for tissue storage. Both food and host factors influence bioavailability of the nutrients. Bioavailability of nutrients in humans is determined by a sequential series of events, which include (1) digestion and release of elements from food matrix into the lumen of gastrointestinal (GI) tract (availability), (2) transport into intestinal enterocytes (uptake), (3) efflux across the basolateral membrane of enterocytes into the circulation (absorption), (4) retention, or endogenous excretion in urine and feces (retention), (5) transport to tissues for use in normal body functions (utilization), and (6) transport to storage sites (body stores) (Fairweather-Tait et al. 2005). Furthermore, Fernández-García et al. (2009) defined bioavailability as a sum of bioaccessibility and bioactivity and defined bioaccessibility as the fraction of a compound that is released from its matrix in the GI tract and thus becomes available for intestinal absorption. Bioaccessibility includes the entire sequence of events that take place during the digestive transformation of food into materials that can be assimilated by the body, the absorption/assimilation into the cells of the intestinal epithelium, and lastly, the presystemic metabolism. Bioactivity includes events linked to how the bioactive compound when and how it is transported and reaches the target tissue, how it interacts with biomolecules, the metabolism or biotransformation that it may undergo, and the generation of biomarker and the physiologic response it causes. There are different analytical approaches and models, which have been advocated to measure bioaccessibility of nutrients and bioactive compounds, and these are described below with respect to iron, zinc, and $\beta$-carotene utilization by humans.

3. Iron and Zinc. Traditionally rats have been suggested as the animal model for performing nutrition studies; however, the rat model has a 
number of limitations that makes extrapolation back to a human situation questionable, including significantly different food intake and energy expenditure for body size, a different life-span and body proportion, differences in intestinal morphology and enteric microbiota, as well as other distinct physiological differences (Gregor 1992). Another major problem with using rat models for mineral studies is their propensity for practicing coprophagy, which may have dramatic impact on nutritional study.

Pigs share many similarities with humans making them a valuable experimental model for nutrient bioavailability and absorption. The difference between human and pig is in their intestine lengths and spatial arrangements of the intestines within the abdominal cavity. The small intestine of adult pig is around $15-22 \mathrm{~m}$, whereas the large intestine has an average length of 4-6 $\mathrm{m}$. In contrast, the small intestine of a human adult averages around $5.5-7 \mathrm{~m}$, whereas the large intestine is around $1.5 \mathrm{~m}$. In spite of these differences, the porcine digestive and metabolic processes function in much the same way as those of humans, and digest transit times are also similar between the two species. The intestinal villus structure and component epithelial cells are also very much alike, which makes pigs an ideal model for human nutritional studies to investigate bioavailability and digestibility of various dietary factors in gastrointestinal compartments (Patterson et al. 2008).

Chickens have a shorter intestinal tract, $2.2 \mathrm{~m}$, relative to humans (Sturkie 2000). The duodenum in chicken is the primary iron absorption site, a feature similar to humans (Sturkie 2000). Chickens could be a relevant model as a source of tissue for in vitro iron bioavailability studies, in vivo feeding trials, or both. Recently, Tako et al. (2009b) evaluated broiler chickens as a model for assessment of iron bioavailability using iron-deficient and -nondeficient (control) birds and a unique duodenal loop technique for direct measurement of iron absorption. They detected higher iron absorption in the iron-deficient birds. In addition, expression of proteins involved in iron uptake and transfer were elevated in the low iron group, and concluded that this model exhibits the appropriate responses to Fe deficiency and has potential to serve as a model for Fe bioavailability.

Haas et al. (2005) used poultry model in plant breeding to select for traits that enhance the nutritional quality of crops by increasing iron concentration or bioavailability or both. The poultry model therefore could serve as an intermediate test of in vivo iron bioavailability in preparation for subsequent human studies.

Several reports indicate phytic/zinc (P/Z) molar ratio a poor indicator of zinc bioavailability as it does not take into account the aggravating 
effects of calcium on zinc absorption in phytic acid containing diets (Forbes et al. 1983, 1984). However, phytate $\times$ calcium/zinc ratio in most of the soybean-based processed foods is a better predictor of zinc bioavailability than the phytate/zinc ratio (Fordyce et al. 1987). Further, Miller et al. (2007) developed a mathematical model of zinc absorption as a function of dietary zinc and phytate, which they tested on select existing data sets to find a good fit $\left(R^{2}=82 \%\right)$ that demonstrate the validity of the model to study zinc nutrition and metabolism and estimate dietary zinc requirements in varied populations. Similarly, the phytate/iron molar ratio has been suggested as an indicator of iron bioavailability in beans (Ariza-Nieto et al. 2007; Pachón et al. 2009).

4. $\beta$-Carotene. Foods containing provitamin A carotenoids ( $\beta$ carotene, $\beta$-cryptoxanthin, and $\alpha$-carotene) are the primary source of vitamin A. Mechanisms regulating bioavailability and bioconversion of carotenoids include (1) the release of carotenoids from the food matrix, (2) the solubilization of carotenoids into micelles, (3) the uptake of carotenoids into intestinal mucosal cells, (4) the absorption of intact carotenoids, (5) the cleavage of provitamin A carotenoids within the enterocytes or within other tissues to yield vitamin $A$, and (6) the tissue distribution, metabolism, and recycling of carotenoids (Castenmiller and West 1998). An ideal model for carotenoids research should be the one that (1) absorb a variety of carotenoids intact at the physiologic levels in the same way as with humans, (2) have carotenoids distribution in tissues similar to that of humans, and (3) represent an appropriate model for the disease state of interest as many diseases in humans have been diagnosed due to vitamin deficiency. Like humans, gerbil, ferrets, and preruminant calves all absorb $\beta$-carotene intact, but only gerbils and calves convert $\beta$-carotene to vitamin $A$ with efficiency similar to that of humans (Lee et al. 1998, 1999; Howe and Tanumihardjo 2006). The gerbils are small, easily maintained in large numbers, and readily available. They are also an established model for cholesterol and lipid metabolism because their serum lipid profile responds to dietary changes similarly to humans (Lee et al. 1999).

The in vitro Caco-2 model have also been suggested to measure bioavailability and absorption of carotenoids including $\beta$-carotene as a rapid and cost-effective model for assessing bioavailability of carotenoids from meals (Garrett et al. 1999; Liu et al. 2004), which varies. widely both for different carotenoids (Reboul et al. 2006; GranadoLorencio et al. 2007; Kean et al. 2008; Failla et al. 2009; O’Sullivan et al. 2010). Alternately, the TNO gastrointestinal tract model (TIM) is a dynamic computer-controlled in vitro system that closely mimics the 
physiological processes occurring within the lumen of the stomach and small intestine of humans (Minekus et al. 1995). The TIM system reproduces (1) three compartments of the human small intestine, (2) chime transit from one digestive compartment to the next, (3) $\mathrm{pH}$ change during the gastric digestion and from the duodenum to ileum, (4) sequential arrival of digestive secretions, and (5) passive absorption of small molecules and water. This model has shown its usefulness in studying the digestive stability of carotenoids from different food matrices throughout the gastrointestinal tract (Blanquet-Diot et al. 2009). Combining the TIM system with Caco-2 cells, Déat et al. (2009) found the potential applicability and predictive value of this in vitro approach to access the bioavailability of bioactive compounds from food or supplements.

\section{B. Factors Influencing Nutrients Bioavailability}

1. Enhancers/Inhibitors. There are two types of dietary iron: nonheme iron that is present in both plant foods and animal tissues, and heme iron that comes from hemoglobin and myoglobin in animal source foods. The heme iron is more readily and rapidly absorbed than nonheme iron. Other factors that influence the iron bioavailability could be broadly grouped as either enhancer or inhibitor of nutrients. The former includes ascorbic acid, meat, fish, and poultry, while the latter phytate, polyphenols, calcium, some plant proteins, wheat bran, and fiber (Kalgaonkar and Lönnerdal 2008; Hurrell and Egli 2010; Petry et al. 2010). Variation in seed color impacts its quality, Lung'aho and Glahn (2010) investigated the effect of seed coat color on iron bioavailability from a Tanzanian complementary food mixture. They detected that white-seeded beans had a significantly higher amount of ferritin but lower amount of tannins when compared to all other porridge ingredients including the red-seeded beans, suggesting that substitution of complementary food ingredients with high antinutrient concentrations (such as colored seeds) with those that have lower antinutrient concentrations (such as white seeds) may improve bioavailability from complementary food home recipes.

Polyphenols in foods may chelate dietary $\mathrm{Fe}$ and lówer its bioavailability. Red beans have higher polyphenols than white-seeded beans. Hu et al. (2006) compared iron bioavailability from colored beans (white, red, pinto, and black beans) using an in vitro digestion Caco-2 cell culture model, while others (Tan et al. 2008; Tako et al. 2009a) used both in vitro and in vivo (pigs) to compare $\mathrm{Fe}$ bioavailability between 
colored beans. The former detected 10 -fold higher bioavailable Fe in white beans, suggesting that color-importing polyphenols inhibit iron absorption, while the latter found no difference in bioavailable $\mathrm{Fe}$ between red and white beans, which is because pigs seem able to adapt to the inhibitory effects of polyphenols on Fe absorption by increasing the secretion of protective protein-rich proteins in their saliva. More recently, Petry et al. (2010) studied the influence of bean polyphenols relative to phytic acid on iron bioavailability in humans (young women). This study revealed lower iron absorption by $14 \%$ with $50 \mathrm{mg}$ polyphenol $(P<0.05)$ and by $45 \%$ with $200 \mathrm{mg}$ polyphenols $(P<0.001)$. The mean iron absorption from whole bean porridge was $2.5 \%$. Polyphenol and phytic acid removal increased absorption 2.6fold $(P<0.001)$ and removal of polyphenol from dephytinized porridge doubled iron absorption $(P<0.001)$. Dephytinization did not increase iron absorption in the presence of polyphenol, but in their absence, absorption increased 3.4-fold $(P<0.001)$, which indicate that both polyphenol and phytic acid should be reduced to enhance iron bioavailability in bean. The lowering only one inhibitor will have a modest influence on iron absorption.

Both garlic and onion are rich sources of dietary sulfur-containing amino acids. Gautam et al. (2010a) reported the enhancing effect of these two species on iron bioaccessibility in cereals $(9.4 \%-65.9 \%$ increase) and legumes (9.9\%-73.3\% increase) in both raw and cooked conditions. These two species similarly enhance the bioaccessibility of zinc from the food grains, the increase ranges from $10 \%$ to $159 \%$ in cereals and from $10 \%$ to $50 \%$ in pulses. This novel information has the potential application in evolving a food-based strategy to improve the bioavailability of minerals and hence contribute to the human health benefit. Malting generally improves the nutrient content and digestibility of foods. Platel et al. (2010) reported increased effect of malting on bioaccessibility of iron by $>$ threefold in finger millet and by $>$ twofold in wheat, whereas no such effect seen in barley; however, malting increased bioaccessibility of zinc to the extent of $234 \%$ in wheat and $100 \%$ in barley. In contrast, malting reduced the bioaccessibility of zinc in finger millet.

Green leafy vegetables and orange/yellow colored fruits are rich source of carotenoids, and a beneficial effect of vitamin $A$ and $\beta$-carotene on enhanced nonheme irons absorption has been reported (García-Casal et al. 1998; Layrisse et al. 2000). The presence of vitamin A increases iron absorption up to 3 times for rice, 2.4 times for wheat, and 1.8 times for maize, while $\beta$-carotene increases iron absorption almost 3 times, showing that both compounds prevent the inhibitory 
effect of phytates on iron absorption (Layrisse et al. 2000). More recently, Gautam et al. (2010b) evaluated $\beta$-carotene-rich vegetables on bioaccessibility of iron and zinc in cereals and legumes-based foods. Addition of carrot or amaranth significantly enhanced the bioaccessibility of iron and zinc from the food grains, the percent increase being 13.8-86.2 in the case of carrot and 11-193 in the case of amaranth. Pure $\beta$-carotene added at an equivalent level also enhanced the bioaccessibility of iron by $19.6 \%-102 \%$ and zinc by $16.5 \%-118 \%$ from the cereals examined. This positive effect of $\beta$-carotene-rich sources on the bioaccessibility of either iron or zinc is generally greater in the cooked grains than the raw grains.

The iron status of the individual, food additives, such as erythorbic acid, and other host-related factors, such as obesity, play a key role in iron bioavailability (Hurrell and Egli 2010). Some unresolved issues that require further investigation include the mechanism by which calcium inhibits iron absorption, the nature of meat factor, the role of inulins on microflora, the influence of vitamin $A$ and carotenoids, and nondigestible carbohydrates on iron bioavailability (Hurrell and Egli 2010). An earlier report revealed the beneficial effect of vitamin A and carotenoids on iron bioavailability (García-Casal et al. 1998).

\section{Production Environment, Postharvest and Storage Conditions, and} Food Processing and Preparation. Burt et al. (2010) studied the effect of postharvest factors on carotenoids concentration and composition in high-carotenoid maize kernels. A preliminary trial using room temperature drying indicated that while carotenoids profiles remain stable during storage, carotenoids levels decrease significantly from initial levels between 3 and 6 months of storage, but then remain stable for another year. Further, a more detailed study using three drying and storage regimes (freeze-drying and storage at $-80^{\circ} \mathrm{C}$; room temperature drying and storage; $90^{\circ} \mathrm{C}$ drying and room temperature storage) reveals that extreme caution is needed to maintain carotenoids levels in maize during handling and storage, but in situations where freeze-drying is not possible, high heat drying is no more detrimental than low heat drying. In OFSP, hot air cross flow drying retained significantly more provitamin A than sun drying, while no significant difference in provitamin A retention between solar and sun drying: The shape of the sweet potato pieces (chip or crimped slice) also influenced provitamin A retention during sun drying: crimped slices retained more provitamin A (Bechoff et al. 2009). Further studies on effect of drying and storage on the degradation of carotenoids in OFSP reveals that carotenoids losses during drying are low (15\% or less) and carotenoids 
retention is not dependent on the type of dryer (solar or sun). However, 4 months of dried sweet potato chips storage at room temperature resulted $\sim 70 \%$ of carotenoids losses, independent on the use of opaque or transparent packaging (Bechoff et al. 2010). Furthermore, Wolbang et al. (2010) reported significant cultivar effects on $\beta$-carotene content, its bioaccessibility, and antioxidant properties in melons. Sowing time significantly affected $\beta$-carotene contents, and antioxidant potentials, but these were highly cultivar dependent, with season having no effect. Postharvest storage over 4 weeks at $7^{\circ} \mathrm{C}$ resulted in losses of antioxidant potential and $\beta$-carotene concentration independent of cultivar. Furthermore, genotypic differences in maturity among maize hybrids were associated with $\beta$-carotene concentration in grain, thus, more research is warranted to study the effects of maturity, environment, and timing of harvest on provitamin A concentrations in grains (Pixley et al. 2011a).

Several traditional food processing and preparation methods including thermal processing, mechanical processing, soaking, fermentation, and germination/malting are reported to significantly enhance the bioavailability of micronutrients in plant-based diets (Erdman and PnerosSchneier 1994; Eyzaguirre et al. 2006; Hotz and Gibson 2007; Hemalatha et al. 2007a,b). Processing has the potential to impact bioavailability of carotenoids (van het Hof et al. 1999; Reboul et al. 2006; Mamatha et al. 2010). Preparation methods (boiling, roasting, temperature and the duration of roasting) also impact both the retention and bioavailability of $\beta$-carotene in cassava (Thakkar et al. 2009); however, steaming/boiling of cassava resulted significantly lower $\beta$-carotene losses than either solar drying or open-air sun drying OFSP slices (Bengtsson et al. 2008). Fermented maize flour is used to prepare porridges and a variety of other staple foods in West Africa. Fermentation provides an optimal $\mathrm{pH}$ for enzymatic degradation of phytate, which may increase the amount of soluble calcium, iron, and zinc, and also contributes to the safety, shelflife, and acceptability of maize-based foods (Blandino et al. 2003). The fermentation does not adversely affect the retention of provitamin A carotenoids in porridges prepared with high $\beta$-carotene maize (Li et al. 2007). Veda et al. (2008) reported the beneficial effect of food acidulants (amchur, lime, tamarind, and kokum) and antioxidant spices (turmeric and onion) on the bioaccessibility of $\beta$-carotene in fleshy and leafy: vegetables. Amchur and lime enhance the bioaccessibility of $\beta$-carotene, both in raw and heat-processed vegetables, with more pronounce effect of lime juice than amchur. In contrast, turmeric significantly enhance the bioaccessibility of $\beta$-carotene, especially when heat-processed vegetables. Onion enhances the bioaccessibility of $\beta$-carotene from the pressure-cooked carrot and amaranth leaf and from open-pan-boiled 
pumpkin and fenugreek leaf. In addition, lime juice, turmeric, and onion minimized the loss of $\beta$-carotene during heat processing of the vegetables.

The presence of other carotenoids in a meal may also adversely affect the bioavailability of provitamin A carotenoids (van den Berg 1999). The absorption of carotenoids from the meal undergo a series of processes that include its initial transfer from food matrix to oil droplets in the gastrointestinal lumen, partitioning of the pigments into mixed micelles for delivery to absorptive epithelial cells during the small intestinal phase of digestion, and the uptake and incorporation of the pigments into chylomicrons secreted into lymph, and in vitro studies support the interactions between carotenoids during preabsorptive processes (Borel et al. 1996; van den Berg and van Vilet 1998; Tyssandier et al. 2001). More recent results revealed that bioaccessibility of provitamin A carotenoids is minimally or not affected by other carotenoids (Davis et al. 2008; Thakkar and Failla 2008).

The preformed vitamin $A$ is readily available from foods, while carotenoids are much more difficult to assimilate. A number of factors have been identified that either enhance or hinder the bioavailability of carotenoids and the bioconversion of food provitamin A carotenoids to vitamin $A$ in humans. These include species of carotenoids, molecular linkage, amount in the meal, matrix (food) properties, effectors, nutrient status, genetic, host specificity; and interactions between factors, often abbreviated as SLAMENGHI (Tanumihardjo 2002; Reboul et al. 2006; Tang 2010). Conversion of $\beta$-carotene (provitamin A) into vitamin A in humans takes place predominantly in the intestine.

\section{Provitamin A ( $\beta$-Carotene) Conversion to Retinol (Vitamin A).}

Wide variation in carotenoid to vitamin A conversion factors, ranging from 3.6:1 to $28: 1$ by weight, not only between studies but also between individuals in a particular study have been reported, which show that the vitamin A value of individual plant foods rich in provitamin A carotenoids may vary significantly, thus, meriting further investigation for the development of dietary guidelines to combat vitamin A deficiency worldwide (Tang 2010). Furthermore, Li et al. (2010b) quantified the vitamin A equivalence in the $\beta$-carotene-biofortified maize porridge consumed by women and found that on average $6.48 \pm 3.51 \mu \mathrm{g}$ of the $\beta$-carotene in $\beta$-carotene-biofortified maize porridge and $2.341 .61 \mu \mathrm{g}$ of the $\beta$-carotene in the reference dose were each equivalent to $1 \mu \mathrm{g}$ of retinol, which suggest that $\beta$-carotene in biofortified maize has good bioavailability as a plant source of vitamin A. Similarly, $\beta$-carotene derived from 'Golden Rice' is effectively converted to vitamin A $(0.24-0.94 \mathrm{mg}$ retinol) in humans. Thus, the conversion factor of Golden Rice $\beta$-carotene 
to retinol is (3.8 \pm 1.7$)-1$ with a range of (1.9-6.4)-1 by weight, or $(2.0 \pm 0.9)-1$ with a range of (1.0-3.4)-1 by moles (Tang et al. 2009).

\section{Efficacy of Biofortified Crops on Human Health}

Selective breeding has resulted into the development of a number of nutritionally enhanced advanced lines and hybrids such as quality protein maize (QPM) (Atlin et al. 2011); mineral-dense (Fe and $\mathrm{Zn}$ ) common bean, maize, pearl millet, rice, and wheat; $\beta$-carotene-rich sweet potato, cassava, maize, and rice, with a few of these already released for cultivation in some countries (see Section VI.E). To date, only few studies have been conducted to assess the bioavailability to humans of micronutrients from these enriched lines/hybrids. A 9-month human efficacy study, involving seed Fe-dense rice line IR68144 and human subjects in the Philippines (192 religious sisters), revealed that the consumption of biofortified rice without any other changes in diet is efficacious (increased body iron by $20 \%$ ) in improving iron stores of women with iron-poor diets in the developing world (Haas et al. 2005; http://nutrition. org/cgi/content/full/135/12/2823). Rosado et al. (2009) compared the intake and absorption of $\mathrm{Zn}$ in adult women who consumed tortillas made either from biofortified or nonbiofortified wheat. The study revealed higher $\mathrm{Zn}$ intake from biofortified wheat $\left(5.7 \mathrm{mgg}^{-1}\right.$ at $95 \%$ extraction and $2.7 \mathrm{mg} \mathrm{g}^{-1}$ at $80 \%$ extraction) compared to that with the corresponding control wheat, while the Zn absorption from biofortified wheat meals at both extraction level was comparable $\left(\sim 2 \mathrm{mgg}^{-1}\right)$, which was $0.5 \mathrm{mg} \mathrm{day}^{-1}$ higher than that from the corresponding control wheat, demonstrating that valuable increases in $\mathrm{Zn}$ absorption can be achieved from biofortified wheat. Several low phytate (lp) mutants have been reported in barley, common bean, maize, rice, soybean, and wheat (see Section V.A). In a study on the effects of Zn absorption among Guatemalan school children fed with lp maize, its corresponding wild type (WT) maize, or local maize, Mazariegos et al. (2006) found variable phytate (lp: 1,536 $\mathrm{mg} \mathrm{d}^{-1}$, WT: $2,056 \mathrm{~d}^{-1}$, local: $2,253 \mathrm{~d}^{-1}$ ) and zinc (lp: $8.6 \mathrm{mgd}^{-1}$, WT: $8.1 \mathrm{mg} \mathrm{d}^{-1}$, local: $9.7 \mathrm{mg} \mathrm{d}^{-1}$ ) intakes, and dietary phytate/Zn molar ratio (lp: 18, WT:26, and local: 23), the corresponding fractional absorptions of zinc (lp: 0.32, WT: 0.28, local: 0.29) and total absorbed zinc (lp: $2.72 \mathrm{mg} \mathrm{d}^{-1}$, WT: $2.30 \mathrm{mg} \mathrm{d}^{-1}$, local: $2.78 \mathrm{mg} \mathrm{d}^{-1}$ ) were similar between the maize groups, which indicates that lp maize did not show an altered efficiency of zinc absorption in this population.

$\beta$-carotene-rich OFSP is an excellent source of provitamin A. In a study conducted on primary school children in south Africa, van Jaarsveld et al. (2005) found that the consumption of OFSP improves vitamin A status, 
which can be used as a viable long-term food-based strategy for controlling vitamin A deficiency in children in developing countries. Further, Low et al. (2007) found that integrated promotion of OFSP contributes to increases in vitamin $A$ intake and serum retinol concentrations in young children in rural Mozambique. The vitamin intake in intervention children was much more than those of control children. A high $\beta$-carotene $\left(15 \mu \mathrm{g}^{-1}\right)$ temperate hybrid, CI7 $\times$ DEexp, is currently being investigated for its efficacy in human and animal (Yan et al. 2010).

Using proxy measures and recipe prepared from nutritionally enhanced bean (NEB) that has more Fe and Zn than conventional bean (CB) and nutritionally enhanced maize (NEM) that has more tryptophan and lysine than conventional maize (CM), Pachón et al. (2009) detected similar Fe in the cooked NEB and CB and in NEM and CM; similar in vitro digestibility of Fe in cooked NEB and CB but greater in NEM than CM; higher Zn in uncooked and cooked NEB than in the CB but lower bioavailability of $\mathrm{Zn}$ due to higher phytate:Zn molar ratios in the cooked NEB and CB. Further, they detected no such differences in $\mathrm{Zn}$ concentration or phytate/Zn molar ratios in the maize recipe. The in vitro protein digestibility was comparable for NEM and CM, but was higher for NEB than for CB, which reveals that nutritionally enhanced crops can improve human nutrition if it translates into more nutrients absorbed and utilized by the body (Pachón et al. 2009). Using meta-analysis and community-based approach, Gunratna et al: (2010) studied the nutritional impact of QPM, which revealed that consumption of QPM instead of conventional maize led to a $12 \%$ increase in the rate of growth in weight and a $9 \%$ increase in the rate of growth in height in infants and young children with mild-to-moderate undernutrition from populations, with maize as staple food. In another study on young children in the Ethiopian highlands, the inclusion of QPM in children's diet could reduce or prevent growth faltering and may in some cases support catch-up growth in weight (Akalu et al. 2010). Clearly, more such studies are needed to assess the efficacy of biofortified crops on human health.

In summary, the Caco- 2 model is the most frequently used in the in vitro assay to assess the bioavailability of micronutrients including $\mathrm{Fe}, \mathrm{Zn}$, and $\beta$-carotene. It is a useful tool for initial screening; however, should be complemented with the in vivo studies. No one animal model is perfect to completely mimic human absorption and metabolism of nutrients; however, pigs share many similarities with humans making them a valuable in vivo model for $\mathrm{Fe}$ and $\mathrm{Zn}$ bioavailability and absorption. For $\beta$-carotene, gerbils and calves are appropriate models as both convert $\beta$-carotene to vitamin A with efficiency similar to that of humans. The food-based diets contain several enhancers/inhibitors that impact 
nutrients bioavailability and absorption. Production environments, postharvest drying/storage, and food processing and preparation methods have potential to adversely impact loss (degrade) and/or bioavailability of $\beta$-carotene. The carotenoids as a whole promote $\mathrm{Fe}$ bioavailability. The preliminary studies involving biofortified crops revealed that consumption of biofortified food is efficacious to improving Fe and Zn levels on humans.

\section{PHENOTYPIC SCREENS}

\section{A. Methodology and Approaches to Screen for Seed Iron, Zinc, Phytate, and $\beta$-Carotene}

To select or breed crop cultivars denser in seed iron, zinc, or $\beta$-carotene, it is important to use standardized methodologies for screening the materials. Such an approach is a prerequisite for comparing results across various locations and sites. It is also important to make sure that the medium of growth (generally soil in a field experiment) is supplied with known amounts of all nutrients including Fe and Zn for growth, development, and seed production as interactions between nutrient uptake and seed content are well-known. Multisite testing will ensure expression of genetic differences, if any; in micronutrients and help determine the heritability and genotype $\times$ environment interaction for each element or vitamin. In addition, soil and environmental factors-especially soil water regime and climatic factors such as temperature-also influence yield and the seed quality relative to $\mathrm{Fe}$ and $\mathrm{Zn}$ composition of a crop (Stewart et al. 2005). Since seed Fe and Zn are liable to contamination during harvest and preparation of the samples (grinding) for analysis in the laboratory using routine methods, extra precautions are needed to avoid contamination with $\mathrm{Fe}$ and $\mathrm{Zn}$ during these operations (Mills and Jones, 1996). Grinding with Teflon chambers or at. least stainless steel or fiber mills is recommended (Stangoulis and Sison 2008; Blair et al. 2009a).

In the literature, results on the mineral composition including Fe, $\mathrm{Zn}$, and $\beta$-carotene of seeds of various crops are reported as concentration (Velu et al. 2006; Graham et al. 2007; Cakmak 2008; Demirkiran 2009; Tiwari et al. 2009) that refers to mass or molar ratio such as mg or moles $\mathrm{kg}^{-1}$ seed. But sometimes, the quantity of nutrients in plant parts or the whole plant is expressed as uptake or content (expressed as $\mu \mathrm{g}$ seed $^{-1}$ or organ). The use of the term concentration and content should be precise so as to refer to the appropriate aspect of nutrient physiology. Uptake and content of nutrients are influenced by dilution; and the 
concentration decreases due to increased dry matter and increases due to a loss of plant dry matter (Jarrell and Beverly 1981). Seed mineral content reflects the supply of that nutrient to the individual growing seedling and is therefore of agronomic importance, while concentration is more important in overall nutritional terms.

Several authors have reported on the yield dilution of seed $\mathrm{Zn}$ and $\mathrm{Fe}$ in various crops including wheat and maize (Oikeh et al. 2003b; Lyons et al. 2005; Pleijel and Danielsson 2009). It is thus suggested that the term concentration ( $\mathrm{mg} \mathrm{kg}^{-1}$ seed) as defined above should be preferred to express minerals in seed of various crops. The content ( $\mu \mathrm{g} \mathrm{seed}^{-1}$ ) of a mineral in seed is influenced by growing conditions that affect seed size. For example, with increased seed size, the amount of the mineral will be diluted compared to low seed size if the amount of mineral nutrient is fixed. Blair et al. (2011) pointed this out in a comparison of content versus concentration among various legumes where model legumes have tended to be small seeded while crop legumes are large seeded, especially as with Andean common beans. This is especially true if there is variability among seed tissues such as the seed coat, embryo, or cotyledonary tissue, unless compensation between these tissues occurs (Ariza-Nieto et al. 2007). For example, micronutrient levels in the embryo and seed coat are much higher than in the endosperm. Hence, seed shriveling, wrinkling, and weathering can result in elevated micronutrient concentrations-the "concentration" effectgiven that the seed coat-to-endosperm ratio is much higher than in normally developed grains (Cakmak et al. 2000; Imtiaz et al. 2003). Assessing the correlation between micronutrient concentration and content can help to determine whether seed size and shriveling affect microhutrient concentration of a given sample of genotypes (Pfeiffer and McClafferty 2007). More recently, Velu et al. (2011) detected highly significant correlation between the concentration and content of grain Fe $(r=0.45, P<0.01)$ and $\mathrm{Zn}(r=0.65, P<0.01)$, which suggest that higher grain $\mathrm{Fe}$ and $\mathrm{Zn}$ concentrations are not necessarily related to small grain size or weight in wheat.

The atomic absorption spectrophotometer (AAS) method has been suggested for routine estimation of $\mathrm{Fe}$ and $\mathrm{Zn}$ in seeds (Sahrawat et al. 2002; Blair et al. 2009b, 2010a,b,2011). An excellent review on the role of atomic spectrometry in plant science has been published elsewhere, and it is important to recognize recent developments in multielemental and speciation analyses in plants with the resulting functional roles of different elements in plant science (Husted et al. 2011). X-ray fluorescence spectrometry is another method that allows identifying a wide range of micronutrients including elements such as $\mathrm{P}$, which is 
indicative of the antinutrient phytate. Inductively coupled plasma (ICP) analysis is more of a gold standard but is expensive and therefore of less utility in breeding especially as it is usually highly correlated with AAS readings (Blair et al. 2009b, 2010c) and is the current method of choice to detect elements such as aluminum, which has been proposed as an indicator of iron contamination (Stangoulis and Sison 2008).

Lorenz et al. (2007) developed a rapid and inexpensive method for measuring phytate and inorganic phosphorus $(\mathrm{Pi})$ concentrations in maize, which provides adequate precision and simplicity to deal with large number of breeder's samples for estimating phytate and Pi levels simultaneously. Estimates obtained from this technique match closely with those obtained from ion exchange methods, and the repeatability of the values across fields suggests that the protocol can be used to make heritable measurements for both phytate and Pi. Near infrared reflectance spectroscopy (NIRS) is another possibility (Blair et al. 2008, 2009a).

The $\beta$-carotene content in seeds of crops is generally analyzed using high performance liquid chromatography (HPLC) as this method is more precise than the colorimetric assays (Bhaskarachary et al. 1995; Rodriguez-Amaya and Kimura 2004). Further, Hulshof et al. (2007) developed a fast screening (in comparison to HPLC) of maize seeds, which allows distinction between lines the low, medium, and high levels of provitamin A carotenoids by semiquantitative analysis without the need of a full.HPLC analysis of all samples, and hence reduces the cost of analysis.

\section{B. Screening Under Optimal or Nutrient-Deficient Conditions}

Germplasm pools are ideal biological resources to mine allelic variation for beneficial traits including seed micronutrients. Rapid and costeffective phenotypic screens significantly impact the potential to developing seed mineral-dense cultivars. Questions are often asked whether to screen germplasm/breeding populations under optimal soil conditions (not deficient in micronutrients), pot culture using mineraldeficient soils or in hydroponics system, or under natural occurring sites deficient in micronutrients. It is feasible to identify seed mineraldense genotypes by evaluating germplasm/advanced breeding lines under optimal soil conditions (see Section V.A); however, it is quite possible that such germplasm may not show any advantage when grown under mineral-deficient soils. Moreover, research to date reveals strong location effects on micronutrients than genotype by environment interaction effects (see Section VI.B). In particular, seed Zn and, less so, Fe is influenced by environmental factors, as indicated also by lower heritability of these minerals when compared with provitamins A (Pfeiffer 
and McClafferty 2007). An alternative approach would be to identify natural occurring sites deficient in micronutrients for germplasm evaluation to identify genotypes efficient in mineral acquisition and/or remobilization from shoot to seeds:

Mapping soils in geographic regions will go a long way to sustain breeding programs for developing seed micronutrient-dense food crops. Some efforts have been made to map geographic regions deficient in soil micronutrients, for example, (macro- and micro-) nutrient-deficient soils in India (Singh 2008) or zinc-deficient soils in Turkey (Cakmak et al. 1996). More such efforts are needed to identify a representative set of regions to initiate targeted breeding programs for developing micronutrient-dense and micronutrient-deficiency adapted food crops. An alternative to this is to (1) initially screen germplasm under optimal soil conditions to identify genotypes containing high seed micronutrients, (2) use potscreening technique (with micronutrient-deficient soils) to characterize seed micronutrient-dense germplasm for nutrient use efficiency (acquisition and remobilization), and (3) evaluate nutrient-efficient germplasm to identify seed mineral-dense germplasm with good agronomic traits.

The link between the soil nutrient deficiency adaptation and seed loading of a micronutrient must be further studied, so that such germplasm may be intercrossed among themselves to select for multiple traits related to increased nutrient uptake and remobilization (from shoot to developing seeds) or crossed with locally adapted cultivars to transfer these traits into adapted genetic backgrounds. Ideally, such breeding populátions should be evaluated under micronutrient-deficient soils. Alternatively, such populations may be advanced under optimal soil conditions by single-seed descent technique, and only at a later stage the advanced breeding lines should be evaluated in micronutrient-deficient soils for agronomic traits including seed micronutrients per se to select those that performed best under such soils. Genotype ranking could be used as a selection criterion to identify materials that rank similar across locations for seed micronutrients and agronomic traits, including seed yield and seed weight. Finally, the relationship of micronutrient uptake with soil $\mathrm{pH}$, macronutrient fertilization, soil organic matter, and presence of other cations should be analyzed for each crop when considering a micronutrient breeding program [Centro Internacional de Agricultura Tropical (CIAT), unpublished].

\section{Plant Traits Associated with Increased Acquisition of Iron and Zinc}

Higher plants acquire $F$ e from the rhizosphere through two strategiesstrategy' I is employed by dicotyledonous and nongraminaceous 
monocotyledonous species, while strategy II is used by graminaceous monocotyledonous species. Strategy I involves the induction of membrane-bound $\mathrm{Fe}$ (III)-chelate reductases that reduce Fe(III) to the more soluble form $\mathrm{Fe}$ (II), followed by uptake of $\mathrm{Fe}(\mathrm{II})$ via $\mathrm{Fe}(\mathrm{II})$ transporters. Strategy II involves the secretion of phytosiderophore (PS) by roots to solubilize soil Fe(III) (Marschner et al. 1986). Rice is an exception in the sense that it possesses both systems for Fe uptake, making it especially Fe efficient (Cheng et al. 2007).

Plant roots and their exudates greatly facilitate the availability of various plant nutrients by bringing them in the soluble form in the soil (Neumann and Römheld 2002; Rengel 2002; Ryan and Graham 2002; Welch and Graham 2004). Plant roots exude a range of organic compounds and inorganic ions into the rhizosphere that play a crucial role in the availability of and acquisition by plants of plant nutrients, especially $\mathrm{Fe}$ and $\mathrm{Zn}$. Equally important are the differences in root exudation among genotypes that differ in tolerance to $\mathrm{Fe}$ and $\mathrm{Zn}$ deficiency. Genotypic differences in nutrient acquisition-an important determinant of nutrient use efficiency-are associated with root size and morphology, root physiology, increased root (adventitious) production, soil volume explored by roots, and the availability of Fe and $\mathrm{Zn}$ in the growing medium (because $\mathrm{Fe}$ and $\mathrm{Zn}$ deficiency in the soil induces exudation) (Rengel 2002; Lynch 2007; Widodo et al. 2010). Further, root surface area can be enhanced through mycorrhizal associations, while the root processes can affect rhizosphere $\mathrm{pH}$ and redox potential (Marschner et al. 1986; Garrido et al. 2006).

A lot of research has been conducted on the role of root exudates on the acquisition, translocation, and utilization of phosphorus $(\mathrm{P})$ by diverse crop species (Lynch 2007). However, there is a paucity of information on the role of roots and root exudates on the acquisition of $\mathrm{Fe}$ and $\mathrm{Zn}$ by crops. The limited literature available indicates that root exudates are indeed important for the acquisition of $\mathrm{Fe}$ and $\mathrm{Zn}$, especially by crops such as chickpea grown on calcareous and alkaline $\mathrm{pH}$ soils in which these nutrients are poorly available due to high $\mathrm{pH}$. Moreover, genetic variability exists for $\mathrm{Fe}$ and $\mathrm{Zn}$ acquisition in chickpea on alkaline $\mathrm{pH}$ soils (Ali et al. 2002), which is associated with the mobilization of Fe and Zn in the rhizosphere via protons, organic acids, and phenolics in dicots and via phytosiderophores (PS) such as nicotianamine in cereals (Rengel 2002; Welch and Graham 2002; Lynch 2007).

More specifically, graminaceous plants including staple cereals such as wheat and sorghum when grown in calcareous soils with lower $\mathrm{Fe}$ and $\mathrm{Zn}$ availability release mugineic acid (MA) family of $P S$ from their roots to enhance uptake and translocation of Fe and $\mathrm{Zn}$ to the leaves and 
seeds (Marschner et al. 1986). Rice, wheat, maize, and sorghum are more susceptible to micronutrient deficiency than barley, with the former secreting only $2^{\prime}$-deoxymugineic acid (DMA), while the latter (barley) in addition to DMA, also release MA, 3-epihydroxy-2'deoxymugineic acid (epi-HDMA), and 3-epihydroxymugineic acid (epi-HMA) under micronutrient-deficient conditions (Kobayashi et al. 2008). When investigating wheat (Triticum aestivum) cultivars and related nonprogenitor Aegilops species for release of PS in vitro under $\mathrm{Fe}$ - and Zn-sufficient and -deficient conditions, Neelam et al. (2010b) detected three to four times higher release of PS in Aegilops species than in wheat cultivars under both nutrient-sufficient and -deficient conditions. Furthermore, the absolute amount of $\mathrm{Fe}$ and Zn under both conditions was nearly three times higher in roots and shoots of Aegilops species than wheat cultivars. The amount of PS released was highly significantly. correlated with $\mathrm{Fe}(r=0.94)$ and $\mathrm{Zn}$ $(r=0.91)$ in roots. The higher amount of both $\mathrm{Fe}$ and $\mathrm{Zn}$ in Aegilops species compared with that of wheat cultivars under deficient conditions reveal that Aegilops species possess an efficient system for the uptake and translocation of these micronutrients to the leaves and ultimately to seeds.

Nozoye et al. (2011) report that the efflux of DMA, the primary phytosiderophore from rice and barley, involves the TOM1 and HVTOM1 genes, respectively, the missing piece in the mechanics of the Fe acquision by graminaceous plants, which reveals that the TOM1 and HvTOM1 proteins are the phytosiderophore efflux transporters. Under conditions of iron deficiency, rice and barley roots express high levels of TOM1 and HVTOM1, respectively, and the overexpression of these genes increased tolerance to iron deficiency. Further, in rice roots, the efficiency of DMA secretion is enhanced by the overexpression of TOM1 and decreased by its repression, providing further evidence that TOM1 encodes the efflux transporter of DMA. Furthermore, Widodo et al. (2010) showed that Zn-deficiency tolerant line RIL46 acquires Zn more efficiently and produces more root biomass than its nontolerant line IR74 at low [Zn] ext under field conditions. This observation they related with the maintenance of root growth and increased root exudation and uptake of $\mathrm{Zn}$ ligand complexes [DMA and low molecular weight organic acids (LMWOA)] at low [Zn] ext, which could possibly be used as potential breeding targets for enhancing Zn concentration in rice seeds (Widodo et al. 2010).

The roots of bread wheats tolerant to $\mathrm{Zn}$ deficiency exude more phytosiderophores than sensitive bread and durum genotypes, that is, greater tolerance to $\mathrm{Zn}$ deficiency among wheat genotypes is 
associated with increased exudation of phytosiderophores and increased Fe uptake by roots, perhaps as a response to a decreased rate of Fe transport to the shoots (Rengel and Römheld 2000). Clearly, more emphasis should be placed toward understanding the role of various plant traits involved in the acquisition of Fe and $\mathrm{Zn}$ by crops.

\section{Iron and Zinc Uptake, Accumulation, and Translocation to Seed and Nonseed Parts}

Soil is the main source of nutrients-including Fe and $\mathrm{Zn}$-for plant growth, productivity, and accumulation in the seed and nonseed parts of the plant. Even organic and mineral fertilizers (except foliar applicants) are applied to supply $\mathrm{Fe}$ and $\mathrm{Zn}$ to plants through the soil and become part of the soil before they are taken up by the growing plants and metabolized in seed and nonseed parts.

The availability of $\mathrm{Fe}$ and its functions in the soil are mostly based on (a) the reversible redox reactions of $\mathrm{Fe}^{2+}$ (ferrous, reduced form of $\mathrm{Fe}$ ) and $\mathrm{Fe}^{3+}$ (ferric, oxidized form of $\mathrm{Fe}$ ), and (b) its ability to form octahedral complexes with various ligands and to vary its redox potential in response to different environments. Fe availability is indeed the function of solubility rather than of its abundance in the soil (Sahrawat 2000; Guerinot 2001; Hell and Stephan 2003; Pirzadah et al. 2010). Zinc deficiency is common in soils with neutral and alkaline $\mathrm{pH}$ (calcareous, saline-sodic, and sodic soils), intensively cropped soils, soils with poor drainage, and lowland rice soils (Marschner 1995; Fageria et al. 2002; Alloway 2009; Koegel-Knabner et al. 2010; Pirzadah et al. 2010).

The ability of plants to translocate $\mathrm{Fe}$ and $\mathrm{Zn}$ in seed is controlled by a homeostatic mechanism in the plant that regulates absorption, translocation, and phloem sap loading-unloading rates of $\mathrm{Fe}$ and $\mathrm{Zn}$ (Marschner 1995; Welch 1995; Mori 1999; Schurr 1999; Hell and Stephan 2003; Borg et al. 2009). Iron homeostasis is especially well controlled due to the redox potential of free iron radicals and due to the high concentration of iron in most acid tropical soils where many of the world's crops originated (maize, common bean, sorghum, chickpea). Zinc, on the other hand, is more often taken up without strict control mechanism due to its generalized deficiency in many tropical and temperate soils, especially those of volcanic or loess origin.

An interaction of micronutrients with macronutrients needed by crops is also of importance. Hao et al. (2007) conducted a pot experiment to study the effects of nitrogen (N) fertilization on the distribution of $\mathrm{Fe}$ and $\mathrm{Zn}$ in rice shoot and seed of two rice cultivars, IR68114 and 
IR64. IR68114 is a sister line of IR64 bred for high seed-Fe density. In comparison to the control ( $0 \mathrm{~N}$ applied), the application of $\mathrm{N}$ fertilizer $\left(80,160\right.$, and $\left.320 \mathrm{~kg} \mathrm{Nha}^{-1}\right)$ increased the concentrations of minerals including $\mathrm{Fe}$ and $\mathrm{Zn}$ in plant parts as a result of their improved transport from roots to shoots with $\mathrm{N}$ application. The two cultivars differed in the accumulation and concentration of micronutrients indicating that the characteristic expression of the two rice genotypes was not controlled by the amount of $\mathrm{N}$ fertilizer added. The concentrations of $\mathrm{Fe}$ and $\mathrm{Zn}$ in brown rice due to $\mathrm{N}$ application increased by $29 \%$ and $16 \%$ for IR64 and by $22 \%$ and $20 \%$ for IR68144. The results of this study suggest that the application of nutrients other than $\mathrm{Fe}$ and Zn-especially $\mathrm{N}$ that is generally universally deficient in soils-is equally important for improving the seed $\mathrm{Fe}$ and $\mathrm{Zn}$ in cereals such as rice. More recently, similar results on the effects of $\mathrm{N}$ application in improving the seed-Fe and $-\mathrm{Zn}$ concentrations were also reported for durum wheat (Cakmak et al. 2010; Kutman et al. 2010). These authors report that $\mathrm{N}$ nutritional status of the wheat plants can have a synergistic impact on root uptake and the deposition of $\mathrm{Fe}$ and $\mathrm{Zn}$ in seed when $\mathrm{N}$, Zn, and Fe are applied together (Alloway 2009). Similar studies with phosphorus (P) and potassium (K) supply are under way in common bean (Blair et al. 2009a). It is known that in situations with multiple nutrient deficiencies, balanced plant nutrition (combined application of all nutrients that are deficient in the soil) is a prerequisite for improving productivity and nutritional quality of seeds of cereals (Rego et al. 2007; Sahrawat et al. 2008; Pirzadah et al. 2010).

Genotypic differences also exist in the allocation of micronutrients such as Fe and $\mathrm{Zn}$ to seed and nonseed parts. For example, Wu et al. (2010) studied the uptake, translocation, and remobilization of Zn absorbed at different growth stages by rice genotypes of different $\mathrm{Zn}$ densities using $\mathrm{Zn}^{68}$ stable isotope tracer. They found that significant differences in Zn allocation existed between two rice genotypes. Higher Zn concentrations were found in seeds, stems, and leaves of cultivar IR68144 than in IR64, but higher Zn was found in roots of IR64. More than half of the $\mathrm{Zn}$ accumulated in the seeds was remobilized before anthesis, and accounted for $63 \%$ and $52 \%$ of the total Zn uptake for IR68144 and IR64, respectively. The results of this study indicate that $\mathrm{Zn}$ density in rice seeds is closely associated with the ability to translocation of $\mathrm{Zn}$ from old tissues to new tissues at both early and late growth stages of the rice crop and with phloem remobilization of $\mathrm{Zn}$ from nonseeds parts, especially leaves and stems to seeds (Wu et al. 2010). These results are in agreement with those reported by Haslett et al. (2001) who found that foliar application of Zn (applied as 
$\mathrm{Zn}^{65}$ labeled isotope tracer) in inorganic and organic form is equally suitable for providing adequate Zn nutrition to the wheat plant, thus demonstrating the phloem transport of $\mathrm{Zn}$ from leaves to roots of the wheat plants.

The results of the study by Haslett et al. (2001) along with those reported by Pearson and Rengel $(1994,1995)$ clearly demonstrate that the transport of $\mathrm{Zn}$ in phloem from the stem and lower leaves and roots is significant. The results also establish that phloem transport of $\mathrm{Zn}$ from leaves and stem to the developing seed is an effective mechanism for the accumulation of $\mathrm{Zn}$ in wheat seeds. It was concluded that $\mathrm{Zn}$ is highly mobile in phloem (Pearson and Rengel 1994, 1995; Haslett et al. 2001). Similarly, the transport of iron in phloem is well studied although the transport mechanism is not well known and may involve citric acid conjugates and/or nicotianamine as a transport system with most of the studies on Fe-transport based on mutants in peas (Grusak 2000, 2002) or Arabidopsis (Grotz and Guerinot 2006), or rice (Takahashi et al. 2001; Cheng et al. 2007; Masuda et al. 2009). Iron-uptake through iron reductase activity (IRA) seems to be important for total seed accumulation of this mineral in common bean (Blair et al. 2010a).

It has also been reported that the amounts of Zn uptake vary among cereals such as rice, wheat, and barley. In some species such as rice, continuous Zn uptake during seed filling and continuity of loading into the endosperm from the xylem might be the key process. Also, continued Zn uptake requires genetically improved uptake capacity along with Zn availability in the soil or the growing medium (Zee and O'Brian 1970; Pearson and Rengel 1995; Krishnan and Dayanandan 2003; Alloway 2009).

In species such as rice, the root-to-shoot transfer appears less important than seed loading. It would seem for rice at least that endosperm loading might be low, not so much because of transport barriers but because of limited uptake or sink capacity in the highly starch-filled cells (Stomph et al. 2009). Hence for rice, the sink capacity needs to be enhanced by increasing the nonstarch to starch ratio in the endosperm through a larger number of cells with slightly reduced size while maintaining seed production (Stomph et al. 2009). Whether the same is true in the case of wheat and barley is an important issue for future study, and obviously there is a need to generate results on the comparative evaluation of these crops with rice (Stomph et al. 2009).

In wheat plants, remobilization from leaves is important for $\mathrm{Zn}$ allocation to seed and $\mathrm{Zn}$ is phloem mobile (Marschner 1995; Pearson and Rengel 1995). On the other hand, in rice, xylemtransported Zn from uptake during seed filling might be more important 
for $\mathrm{Zn}$ accumulation in seed than phloem-transported Zn remobilized from leaves (Ziang et al. 2007, 2008). It would seem that contrary to wheat (Zee and O'Brian 1970), there is no xylem discontinuity in the rice plant (Zee 1972; Krishnan and Dayanandan 2003). Since rice plant xylem is continuous, Zn can be loaded directly from the xylem in the vascular bundle to the nucellar epidermis and aleurone cells. In dicotyledonous legumes where the pod wall is continuous with stem xylem but the seed embryo is not this may vary. Iron uptake in the meantime is well understood but its transport through phloem and xylem less so. This is especially important in the case of seed loading of iron, where the mechanism is almost completely unknown but must be controlled by a mixture of sink strength and supply. Remobilization from leaves to stem and then to seeds involves the breakdown and creation of different ferritins expressed in each tissue.

It is clear from this discussion that research on the uptake, translocation, and deposition of minerals such as $\mathrm{Fe}$ and $\mathrm{Zn}$ in seed has focused mainly on wheat, rice, and to some extent, maize to understand the mechanisms involved in the biofortification of crops. There is hardly any information, although needed for understanding the process of biofortification, on these aspects for other important food crops, including pearl millet and sorghum in the semiarid tropical regions of Asia and Africa. Obviously, there is a need to investigate the process of seed loading for other cereals as the information would be useful to ascertain differences caused by the differences in the anatomy (Thorne 1985).

In addition to the physiological mechanisms involved in the uptake, distribution, and accumulation of $\mathrm{Fe}$ and $\mathrm{Zn}$ in seed (Borg et al. 2009; Cakmak et al. 2010), the use of genes (Lönnerdal 2003; Vasconcelos et al. 2003; Ghandilyan et al. 2006; Brinch-Pedersen et al. 2007; Waters et al. 2009; Sperotto et al. 2010), and chelating agents such as EDTA (Nowack et al. 2008) have been reported to enhance efflux of nutrients including $\mathrm{Fe}$ and $\mathrm{Zn}$ from the vegetative tissues to seed of crops.

\section{E. Nutrient Use Efficiency}

Micronutrient availability in the soil, especially of $\mathrm{Fe}$ and $\mathrm{Zn}$, is influenced by a range of soil, plant, and climatic factors (Fageria et al. 2002). The $\mathrm{Fe}$ and $\mathrm{Zn}$ deficiency-related problems in various soil orders are further influenced by the nature of the plant material from which the soil is developed (Dudal 1976). Nutrient-use efficiency consists of two components, one related to crop productivity and the second that emphasizes the internal nutrient requirements for a crop or 
genotype. Relative to productivity, nutrient-use efficiency may be defined as the ability to produce higher yield in a soil that otherwise would limit the productivity of a standard or control genotype (Marschner 1995). In terms of agronomic efficiency, it can simply be defined as yield per unit of nutrient ( $\mathrm{Fe}$ and $\mathrm{Zn}$ in this case). Relative to internal requirement or the physiological efficiency for a nutrient, it can be defined as the yield (economic or total) per unit uptake of the nutrient (for detailed discussion, see Gourley et al. 1994; Marschner 1995; Baligar et al. 2001; Rengel 2001; Fageria et al. 2008; Khoshgoftarmanesh et al. 2010). For the purpose of this section for selecting nutrient ( $\mathrm{Fe}$ and $\mathrm{Zn}$ ) rich seeds, we interpret the $\mathrm{Fe}$ and $\mathrm{Zn}$ efficiencies of a crop or genotype in terms of seed concentrations of these nutrients. Harvest index (HI) as defined as a component of seed yield is the ratio of seed mass by total biomass, while $\mathrm{Fe}$ or $\mathrm{Zn} \mathrm{HI}$ is the ratio of $\mathrm{Fe}$ or $\mathrm{Zn}$ concentration in the seed divided by the total concentration of $\mathrm{Fe}$ or $\mathrm{Zn}$ in the biomass. It is thus the Fe or $\mathrm{Zn} \mathrm{HI} \mathrm{that} \mathrm{matter} \mathrm{when} \mathrm{selecting}$ for seed mineral-dense crops: higher HI for Fe and Zn more of these nutrients in seeds. To breed seed mineral-dense crops with high productivity, selection should be based on combining high $\mathrm{HI}_{\text {seed yield }}$

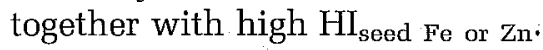

In practical terms when grown on a soil with low availability of Fe or $\mathrm{Zn}$, a micronutrient-efficient genotype acquires and uses a higher amount of these minerals for seed yield as compared to the inefficient genotype, which can be termed nutrient acquisition and use efficiency, respectively. These genotypic differences can arise from greater uptake or greater allocation of the micronutrients to the seed where they allow for better seedling establishment (Baligar et al. 2001; Fageria et al. 2008; Khoshgoftarmanesh et al. 2010). In calcareous soils, rye, triticale, bread, and durum wheat showed differential response to Zn efficiency, rye being exceptional in its high Zn efficiency, followed by triticale $>$ bread wheat $>$ durum wheat, which could be attributed to its greater $\mathrm{Zn}$ uptake capacity from soils (Cakmak et al. 1997b; Erenoglu et al. 1999). The exceptionally high Zn efficiency of rye is because several of its chromosomes, particularly $1 \mathrm{R}$ and $7 \mathrm{R}$, carry the genes controlling Zn efficiency, which are transferable into wheat and can be used for development of new wheat cultivars with high Zn efficiency for Zn-deficient soils (Cakmak et al. 1997a). Further, domesticated emmer wheat accessions such as 3717,19385 , and 22287 were reported to be more Zn efficient (g dry matter/Zn concentration) than modern durum and bread wheats (Genc and McDonald 2008).

Fageria and Baligar (2005) conducted a greenhouse study to evaluate Zn-use efficiency of 10 upland rice genotypes on an Oxisol under 
two $\mathrm{Zn}$ treatments: no $\mathrm{Zn}$ and application of $10 \mathrm{mg} \mathrm{Zn} \mathrm{kg}^{-1}$ soil as zinc sulfate. The results revealed that shoot dry weight, seed yield, $\mathrm{HI}_{\mathrm{Zn}}$, Zn concentration in shoot and in seed were significantly influenced by soil $\mathrm{Zn}$ level and varied by genotype. However, $\mathrm{HI}_{\text {seed yield was }}$ significantly affected only by genotype. Genotypes also differed significantly in Zn recovery efficiency and on average $13 \%$ of the applied Zn was recovered by upland rice genotypes. Seed-Zn concentration

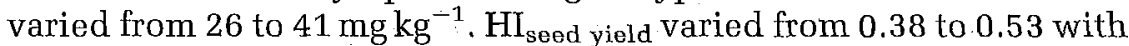
an average value of 0.46 . The $\mathrm{HI}_{\mathrm{Zn}}$ varied from 0.40 to 0.76 with an average value of 0.60 under no application of $\mathrm{Zn}$ fertilizer; $\mathrm{HI}_{\mathrm{Zn}_{n}}$ decreased with applied $\mathrm{Zn}$, and varied from 0.21 to 0.37 with a mean value of 0.28 . The results show that upland rice genotypes differ significantly in seed yield, seed $\mathrm{Zn}$ and $\mathrm{HI}_{\mathrm{Zn}}$ both with and without the application of $\mathrm{Zn}$.

In experiments with rice conducted under controlled conditions, Jiang et al. (2008) reported that with increasing $\mathrm{Zn}$ supply, the $\mathrm{Zn}$ concentration in all individual plant organs increased, but the increase in Zn concentration in stems and rachis was much larger than in seeds. Over a range of added $\mathrm{Zn}$, the $\mathrm{Zn}$ concentration in stems increased from 20 to $400 \mathrm{mg} \mathrm{kg}^{-1}$, but the concentration in the brown rice increased only from 20 to $50 \mathrm{mg} \mathrm{kg}^{-1}$. The $\mathrm{HI}_{\mathrm{Zn}}$ in the tested rice cultivars decreased considerably with increasing total plant $\mathrm{Zn}$ content probably due to concentration effect (opposite of dilution). These results are consistent with those reported earlier for wheat (Herren and Feller 1994) and rice (Fageria and Baligar 2005). Furthermore, Jiang et al. (2008) demonstrated that due to physiological regulation and barriers in the rice plant, it is difficult to enhance Zn concentration in brown rice by simply increasing $\mathrm{Zn}$ supply in the soil or growing medium. It has been proposed that while the breeding target could be to enhance the level of maximum Zn accumulation in the overall plant, further research should focus into the exact tissues in which the regulation of $\mathrm{Zn}$ translocation to seed is strongest and into the genes involved in the regulation mechanism for zinc transport (Jiang et al. 2008).

Sahrawat (2000) determined the amounts of macro- and micronutrients removed by cultivars in a field experiment conducted with an upland rice cultivar, WAB 56-50, under rainfed upland or a lowland rice cultivar, Bouake 189, under irrigated lowland condition's in Ivory Coast, West Africa. The nutrient $\mathrm{HI}$ was highest for $\mathrm{P}$ (0.69) and lowest for $\mathrm{K}(0.10)$. Both $\mathrm{HI}_{\mathrm{Fe}}$ and $\mathrm{HI}_{\mathrm{Zn}}$ were greater for the lowland rice $\left(\mathrm{HI}_{\mathrm{Fe}} 0.46\right.$, $\mathrm{HI}_{\mathrm{Zn}}$ 0.50) as compared to the upland rice cultivars $\left(\mathrm{HI}_{\mathrm{Fe}} 0.21, \mathrm{HI}_{\mathrm{Zn}}\right.$ 0.38). Clearly, the lowland rice ecology provides a better growing soil 
environment than does the upland ecology for rice growth and yield and Fe or Zn accumulation by the crop. Furthermore, it has been demonstrated that the availability of nutrients especially of $\mathrm{Fe}$ and $\mathrm{Zn}$. to rice in soils is greatly influenced by water regime (including flooding) and the availability of $\mathrm{Fe}$ and $\mathrm{Zn}$ is generally favorably affected in the lowland irrigated agroecologies (Ponnamperuma 1972; Gao et al. 2006; Sahrawat 2007, 2009).

In summary, standardized methodology for rapid and effective screens of germplasm pool is a prerequisite for identifying and developing crop cultivars dense in seed $\mathrm{Fe}$ and $\mathrm{Zn}$ under well-defined or optimum nutrient conditions, especially nutrients other than $\mathrm{Fe}$ and $\mathrm{Zn}$. The various screening methods used vary from those conducted under controlled conditions to real world practical conditions in the field, using soil as the substrate. Ideally, the amounts of other nutrients (other than Fe and $\mathrm{Zn}$ ) including major, secondary, and micronutrients are kept in the optimum, while the concentrations of Fe or Zn cover the entire deficiency to the sufficiency range. For practical breeding purposes, identification of plant traits associated with increased acquisition of $\mathrm{Fe}$ and $\mathrm{Zn}$ from the growing medium, generally soil, is of critical importance. The selection of traits varies with soil type, especially with soil $\mathrm{pH}$ (acidic to alkaline soil reaction) and soil water status. Despite diverse soil and agroclimatic conditions under which crops or their cultivars are screened for grains denser in $\mathrm{Fe}$ and $\mathrm{Zn}$, it has been established that genotypes indeed vary in Fe and $\mathrm{Zn}$ uptake, accumulation, and translocation to seed and nonseed parts of crops. To date, the research on uptake, translocation, deposition, and use efficiency of minerals such as Fe and $\mathrm{Zn}$ has been mostly confined to wheat, rice, and to a lesser extent maize. Little information, although urgently needed to understand the process of biofortification, is available for other important food crops such as sorghum and pearl millet. Future in-depth basic research need to focus particularly on understanding the process of seed loading especially in cereals other than wheat and rice, as such insights would help to ascertain differences in seed Fe and $\mathrm{Zn}$ associated with the differences in the anatomy. Rapid, simple, and cost-effective methods for routine determination of $\beta$-carotene, $\mathrm{Fe}$ and $\mathrm{Zn}$ are needed so that a large number of germplasm/breeding lines can be screened and information generated in a timely manner. XRF is an effective assay for initial screening of seed $\mathrm{Fe}$ and $\mathrm{Zn}$ to discard lines in the lower range, while semiquantitative analysis instead of HPLC may be used to discriminate lines for variation in $\beta$-carotene; however, promising lines from these initial screens must be analyzed by ICP or AAS assays for $\mathrm{Fe} / \mathrm{Zn}$ or by HPLC for $\beta$-carotene. 


\section{MINING GERMPLASM COLLECTIONS FOR NATURAL VARIATION FOR SEED IRON, ZINC, AND PHYTATE}

\section{A. Variation and/or Bioavailability of Seed Iron, Zinc, and Phytate}

\section{Seed Iron and Zinc Concentration. Natural variation in plant} genetic resources provides the basic raw material and plays a fundamental role in crop improvement programs. Published evidence on screening of germplasm revealed substantial variation in seed-Fe and -Zn concentrations in common bean, maize, pearl millet, rice, sorghum, and wheat (Table 3.1). For example, higher Fe in some accessions of common bean germplasm from Colombia, Chile, Peru, Rwanda, and Tanzania has been identified; high $\mathrm{Fe}$ and $\mathrm{Zn}$ was found in maize germplasm from southern Africa; high $\mathrm{Fe}$ and $\mathrm{Zn}$ in pearl millet was identified in Iniadi landraces from West Africa; high Fe reported in farmer's preferred sorghum varieties from Benin; traditional rice cultivars were found to contain more $\mathrm{Fe}$ and $\mathrm{Zn}$ than modern cultivars; and high seed-Fe and -Zn was found in einkorn wheat, wild emmer wheat, and species with $\mathrm{S}$ and D genomes. Gene banks in CGIAR centers hold large collections of both cultivated and wild relatives of their mandate crops, with CIAT, CIMMYT, ICARDA, ICRISAT, IRRI, and Africa Rice Center together maintain a total of $7,41,319$ accessions of 3,446 species of 612 different genera (http://singer.cgiar.org/). Only a fraction of the germplasm preserved in gene banks has been screened for seed-Fe and -Zn concentrations (or contents). Clearly, there is a greater need to assess for natural genetic variability locked in these germplasm collections. However, in most cases, it will not be possible for any institution to screen the entire germplasm collection of a given species because of enormous cost and technical manpower associated with the analysis of seed samples for chemical characteristics. Forming core and/or mini-core collections is one way to sample the representative variability from the entire collection of a given species, thus providing an entry point for a wider search in the entire collection (Brown 1989; Upadhyaya and Ortiz 2001). Such subsets have been reported for most major cereal and legume crops (reviewed in Dwivedi et al. 2005, 2007), and should be evaluated to assess the range of genetic variation in seed $\mathrm{Fe}$ and $\mathrm{Zn}$ for use in crop breeding. Islam et al. $(2002,2004)$ analyzed a partial core collection of common bean for variability in Fe and Zn traits while Blair et al. (2010b) did the same for a Rwandan collection that was conserved prior to genocide in that country. Astudillo and Blair (2008), meanwhile, evaluated the Fe and Znconcentrations of all released bush bean varieties in Colombia as a first approximation of micronutrient variability in local germplasm. Likewise, 
Table 3.1. Natural genetic variation for seed-Fe and $-\mathrm{Zn}$ concentrations in common bean, groundnut, maize, pearl millet, rice, sorghum, and wheat germplasm and cultivars covering a period from 1997 to 2010 .

Summary of the variation in

Germplasm evaluated seed mineral content

Range variation

References

\section{Common bean}

29 U.S. grown cultivars and CLAT breeding lines

365 Rwandan landraces

29 U.S. cultivars/high Fe lines

155 Lines from Portugal

90 Lines from Tanzania

\section{Lines from Chile}

>1,000 Germplasm (mostly CIAT core)

Groundnut

9 Diverse lines

Maize

$>1,800$ Germplasm
Fe 8.9-112.9 and Zn 30.9$64.6 \mathrm{mg} \mathrm{kg}^{-1}$

Fe 45.3-95.6 and Zn 25.1-49.1 ppm

$\mathrm{Fe}$ 30.9-64.6 and $\mathrm{Zn}$ 8.9-112.9 ppm

$\mathrm{Fe}$ 32.2-88.4 and $\mathrm{Zn} 11.5-45.3 \mathrm{ppm}$

$\mathrm{Fe} 23.6-105.5$ and $\mathrm{Zn} 19.0-$ $56.1 \mathrm{ppm}$

Fe 68.9-152.4 and Zn 27.9$40.7 \mathrm{mg} \mathrm{kg}^{-1}$

Fe 34-89 and Zn 21-54 $\mu \mathrm{g} \mathrm{g}^{-1}$; Peru germplasm exceptionally high iron (averaged $>100 \mathrm{\mu gg}^{-1}$ )

Fe 13.4-17.9 and Zn 25.2$29.8 \mu \mathrm{gg}^{-1}$

Landraces: Fe 17.5-58.5 and $\mathrm{Zn}$ $14.9-29.7 \mathrm{mg} \mathrm{kg}^{-1}$

Southern Africa: Fe 16.4-63.2 and Zn $12.9-57.6 \mathrm{mg} \mathrm{kg}^{-1}$
Fe 104 and $\mathrm{Zn} 33.7 \mathrm{mg} \mathrm{kg}^{-1}$

$\mathrm{Fe} 50.3$ and $\mathrm{Zn} 24.0 \mathrm{ppm}$

Fe 33.7 and $\mathrm{Zn} 104 \mathrm{ppm}$

$\mathrm{Fe} 56.2$ and $\mathrm{Zn} 33.8 \mathrm{ppm}$

$\mathrm{Fe} 81.9$ and $\mathrm{Zn} 37.1 \mathrm{ppm}$

Fe 83.5 and $\mathrm{Zn} 12.8 \mathrm{ppm}$

Fe 55 and $\mathrm{Zn} 33 \mu \mathrm{gg}^{-1}$

$\mathrm{Fe} 4.5$ and $\mathrm{Zn} 4.6 \mu \mathrm{gg}^{-1}$

Phan-Thien et al. 2010

Landraces: $\mathrm{Fe} 41$ and $\mathrm{Zn}$ $14.8 \mathrm{mg} \mathrm{kg}^{-1}$

Southern Africa: Fe 46.8 and Zn $44.7 \mathrm{mg} \mathrm{kg}^{-1}$
Akond et al. 2011

Blair et al. 2010c

Talukder et al. 2010

Pinheiro et al. 2010

Tryphone and

Nchimbi-Msolla

2010

Paredes et al. 2009

Graham et al. 1999

Bänziger and Long 2000 
Pearl millet

90 Lines

10 Accessions

Rice

202 Modern/traditional cultivars

25 Germplasm

35 Upland cultivars

Wild Oryza

12 Diverse lines including wild relatives

40 Commercial varieties

Traditional varieties

1,138 Germplasm
Fe 30.1-75.7 and $\mathrm{Zn}$ $24.5-64.8 \mathrm{mg} \mathrm{kg}^{-1}$, Iniari landraces high in $\mathrm{Fe}$

Fe $70^{-}-180$ and $\mathrm{Zn} 53-70 \mu \mathrm{gg}^{-1} \quad$ Fe 110 and $\mathrm{Zn} 17 \mu \mathrm{gg}^{-1}$

Traditional cultivars: Fe 38.9 and $\mathrm{Zn} 35.6 \mathrm{mg} \mathrm{kg}^{-1}$; modern cultivars: $\mathrm{Fe} 16.5$ and $\mathrm{Zn}$ $22.4 \mathrm{mg} \mathrm{kg}^{-1}$

Fe 344 and $\mathrm{Zn} 30 \mathrm{ppm}$

$\mathrm{Fe} 16.9$ and $\mathrm{Zn} 20.7 \mathrm{mg} \mathrm{kg}^{-1}$

Brown rice $\mathrm{Fe} 33$ and $20 \mu \mathrm{gg}^{-1}$ in milled rice; brown rice $\mathrm{Zn}$ 21 and $18 \mu \mathrm{gg}^{-1}$ in milled rice

Fe 12.0 and $\mathrm{Zn} 25.3 \mu \mathrm{gg}^{-1}$

Fe 7.8 and $\mathrm{Zn} 17 \mathrm{ppm}$

Fe 12 and $Z n 12 \mathrm{ppm}$

Fe 9-21 and $\mathrm{Zn} 14-36 \mathrm{ppm}$

Fe 6.3-24.4 and $\mathrm{Zn} 13.5-$

$58.4 \mu \mathrm{g} \mathrm{g}^{-1}$; more Fe in aromatic rice/traditional cultivar,

Jalmagna
Velu et al. 2007

Abdalla et al. 1998

Anandan et al. 2011

Singh et al. $2010 \mathrm{~b}$

Moraes et al. 2010

Jiang et al. 2009

Chandel et al. 2010

http://webapp.ciat. cgiar/epmr_ciat/pdf/ poster_19_epmr07pdf Graham et al. 1999;

Gregorio et al. 2000 


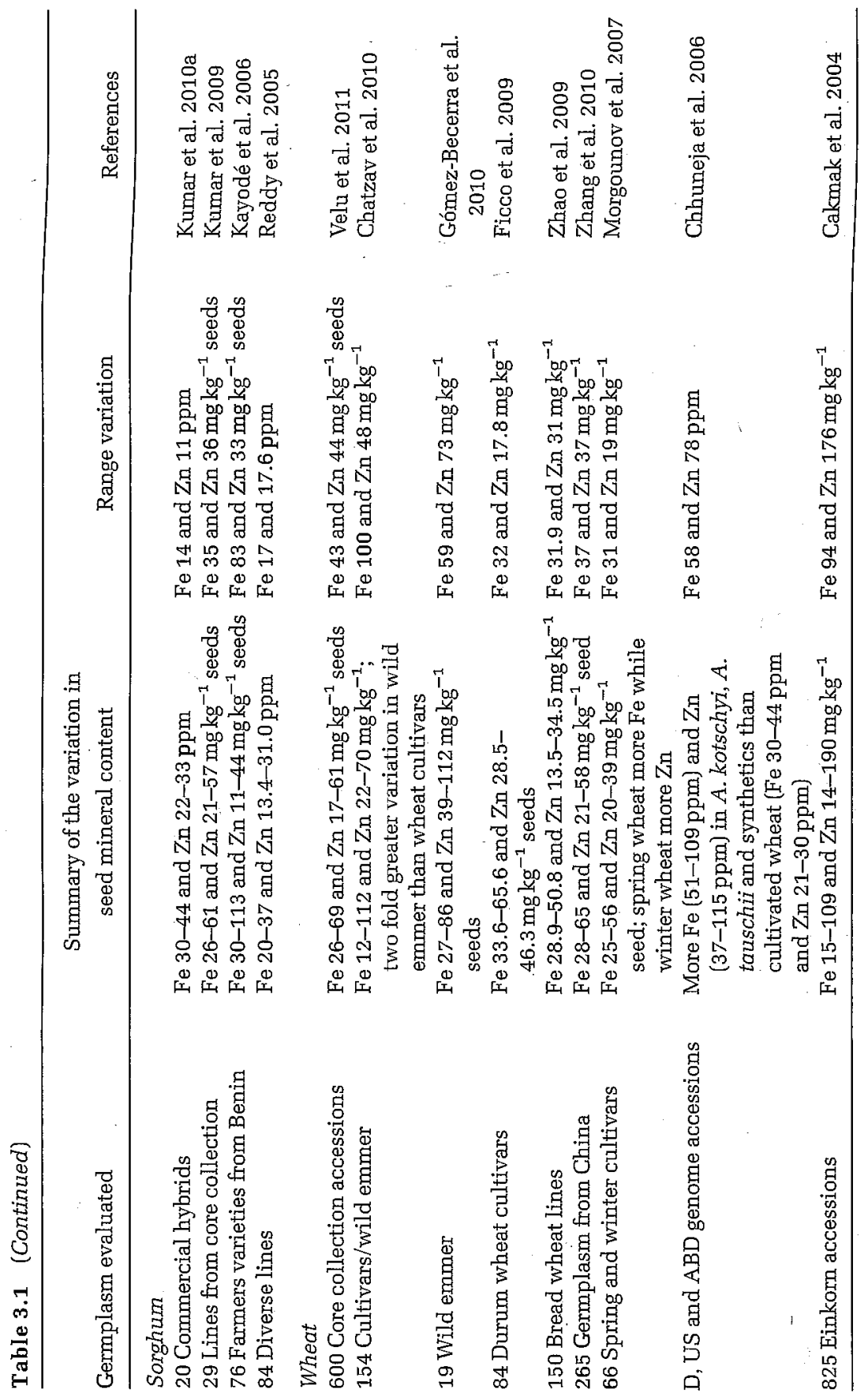


the seed-Fe and -Zn concentrations among selected sorghum core collection accessions varied, respectively, from 26 to 60 and 21 to $57 \mathrm{mg} \mathrm{kg}^{-1}$ seeds compared to controls (Fe $40 \mathrm{mg} \mathrm{kg}^{-1}$ and $\mathrm{Zn} 24 \mathrm{mg} \mathrm{kg}^{-1}$ seeds) (Kumar et al. 2009).

Another approach would be to explore the genetic variation of mineral concentration in germplasm from geographic regions deficient in soil micronutrients, given that germplasm from such regions is expected to develop inherent adaptation mechanisms that favor enhanced nutrient uptake, transport, distribution, and relocation in plants/seeds. This approach, which is also known as habitat characterization or focused identification of germplasm selection (FIGS) (Street et al. 2008), has been successfully employed to characterize plant habitats and species' adaptive responses to temperature, day length, and stresses (Berger 2007; Kaur et al. 2008; Bhullar et al. 2009; El Bouhssini et al. 2009; Berger et al. 2011).

Wild and weedy relatives of common bean and wheat have shown large variability for seed-Fe and -Zn concentrations (Cakmak et al. 2000; Guzmán-Maldonado et al. 2000; Chhuneja et al. 2006; Acosta-Gallegos et al. 2007; Xie and Nevo 2008). For example, the 75 wild and weedy common beans from Jalisco and Durango state of Mexico showed large variability for seed $\mathrm{Ca}\left(500-7470 \mathrm{mg} \mathrm{kg}^{-1}\right)$, iron (64-280 $\mathrm{mg} \mathrm{kg}^{-1}$ ), and zinc (11-33 $\mathrm{mg} \mathrm{kg}^{-1}$ ) concentrations (Guzmán-Maldonado et al. 2000) or nonprogenitor Aegilop species of wheat with large variability for grain $\mathrm{Fe}$ and $\mathrm{Zn}$ (Chhuneja et al. 2006).

Cheng et al. (2007) isolated a point mutation in a gene encoding nicotianamine aminotransferase (NAAT1), which disrupted strategy II system of rice due to the loss of a functional NAAT enzyme, and the naat1 mutant exhibited 3.8-fold higher Fe than the WT. More importantly, the $\mathrm{Fe}$ concentration of the polished seed of the naat1 mutant from field grown rice plants was $4.6 \mathrm{mg} \mathrm{kg}^{-1}$, that is, 3.8 -fold higher than the wild type approaching that of the highest naturally existing lines (Barry 2006). This rice mutant-along with other natural occurring high seed-Fe concentration germplasm-is an ideal resource to enhance seed Fe into the improved genetic background.

2. Seed Phytate Concentration. Phytic acid (myo-inositol-1,2,3,4,5,6hexakisphosphate or InsP6) is the major form of phosphorus storage, which accounts for $65 \%-85 \%$ of the total phosphorus in mature seeds (Raboy 1997). High concentration of phytic acid in foods limits micronutrient bioavailability as it binds minerals ( $\mathrm{Ca}, \mathrm{Fe}$, and $\mathrm{Zn}$ ) to form mixed salts (phytin), largely' excreted by humans and nonruminant animals. The excreted phytin significantly impacts water pollution 
(Lott et al. 2000; Raboy 2001). However, phytic acid is vital for seed development, seedling growth, and development and may have a positive role as antioxidant and anticancer agent (Oatway et al. 2001). The development of lp seed crops is one of several ways to improve the nutritional quality as well as developing environmentfriendly, sustainable production systems. Low phytic acid crops may offer improved nutrition for human populations that largely depend upon cereal- and legume-based staple foods.

Efforts have been made to reduce the levels of phytate in seeds by exploiting natural genetic variation as well by induced mutations. Studies of germplasm collections have revealed significant genetic variation for both seed phytate and/or total $\mathrm{P}$ in common bean, lentil, sorghum, soybean, and wheat (Lolas and Markakis 1975; Raboy et al. 1991; Israel et al. 2006; Kayodé et al. 2006; Ficco et al. 2009; Thavarajah et al. 2010; Akond et al. 2011). Likewise, researchers have used both chemical and physical mutagens to isolate lp mutants in barley (Raboy 2000; Bowen et al. 2006), common bean (Campion et al. 2009), maize (Raboy 2000; Badone et al. 2010), rice (Larson et al. 2000; Raboy 2000; Rutger et al. 2004; Liu et al. 2007; Kim et al. 2008), soybean (Wilcox et al. 2000; Walker et al. 2006), and wheat (Guttieri et al. 2004). These studies reported two types of mutants, those with moderate reduction $(50 \%-65 \%)$ or extreme reduction (95\%) in phytic acid, with the latter being homozygotes and lethal. The total seed phosphorus in these mutants was unaffected, while reduction in phytic acid resulted in corresponding increases in inorganic $\mathrm{P}$ in the seed. However, lp mutants, in general, had reduced germination and seedling development and yielded low compared to WT (Meis et al. 2003; Pilu et al. 2003; Oltmans et al. 2005; Shi et al. 2007), with some exception in barley (Bregitzer and Raboy 2006), soybean (Yuan et al. 2007), and common bean (Campion et al. 2009). Badone et al. (2010) isolated a low phytic acid mutant in maize, $1 p 1-241$, with a reduction of up to $90 \%$ of phytic acid and strong pleiotropic effect on the whole plant including higher level of anthocyanins as compared to wild type either in the embryo ( $\sim 3.8$-fold) or in the aleurone layer $(\sim 0.3$-fold $)$. These mutants could help to discover the carriers and the regulatory mechanisms involved in the vascular transport of plant cell and xenobiotic molecules involved in several fundamental processes, which so far are not fully understood. More recently, Akond et al. (2011) reported large variation in phytic acid (12.52-316.42 $\mathrm{mg} \mathrm{kg}^{-1}$ ) among the 29 U.S. grown cultivars and CIAT breeding lines in common bean, with low phytic acid genotypes such as JaloEEP558, Vista, Xan176, Albion, Voyger, and G122 having high levels of minerals ( $\mathrm{Ca}, \mathrm{Fe}, \mathrm{Zn}$ ) concentration. Large germplasm collections of the major food crops are preserved in gene banks 
globally, but only a fraction of these collections have been screened for low seed phytate. It is reasonable to believe to find genetic variants in these germplasm collections that have low phytate and acceptable agronomic performance. Screening reduced subsets, such as core or mini-core subsets, could be seen as a starting point to mine variation for low seed phytate in germplasm collections.

3. Seed Iron and Zinc Bioavailability. Phytic acid is the major contributor to reduced bioavailability of $\mathrm{Fe}$ and $\mathrm{Zn}$ in cereals and legumes. Few germplasm lines with exceptionally high seed-Fe and/or -Zn concentrations have been reported in common bean, maize, pearl millet, rice, sorghum, and wheat (Table 3.1). However, very little is known about how much of the seed $\mathrm{Fe}$ or $\mathrm{Zn}$ is bioavailable for absorption. Using a range of common bean genotypes differing in seed-Fe concentrations and rat model for bioaccessibility, Welch et al. (2000a) detected large differences in Fe bioavailability, ranging from $53 \%$ to $76 \%$ of total $\mathrm{Fe}$, with higher seed-Fegenotypes resulting in increased amounts of total bioavailable Fe. Likewise, Zn bioavailability in wheat genotypes ranged from $60 \%$ to $82 \%$ (Welch et al. 2000b). Significant differences in seed Fe and/or Zn bioavailability were also reported for maize germplasm (Oikeh et al. 2003a,b, 2004a; Šimic et al. 2009). For example, Oikeh etal. (2003a) detected large variation in $\mathrm{Fe}$ bioavailability that ranged from $30 \%$ below to $88 \%$ above the reference control cultivar, TZB-SR. In some maize cultivars with high seed-Fe and $-\mathrm{Zn}$ concentrations $\left(22-24 \mathrm{mg} \mathrm{kg}^{-1}\right)$ the bioavailable $\mathrm{Fe}$ was $24 \%-36 \%$ higher than the reference control (Oikeh et al. 2004a). Wheat Aegilops species and their derivatives are reported to possess high grain Fe and $\mathrm{Zn}$ concentrations (Chhuneja et al. 2006; Neelam et al. 2010a; Tiwari et al. 2010). More recently, Salunke et al: (2011) detected larger bioavailable Fe among wheat Aegilops derivatives selected for high grain Fe and protein concentrations. The bioavailable $\mathrm{Fe}$ among these derivatives increased up to 1.5 -fold, corresponding to a 1.5- to 2.2-fold increase observed in grain Fe over control. Clearly, more studies are needed to identify genotypic variation for seed $\mathrm{Fe}$ and $\mathrm{Zn}$ bioavailability in order to select genotypes, which have not only high seed-Fe and -Zn concentrations but also more bioavailable $\mathrm{Fe} / \mathrm{Zn}$ for absorption.

\section{B. Distribution of Iron and Zinc in the Seed}

Understanding the accumulation and distribution of essential nutrients in the seed is of primary importance for improving the nutritional quality of staple crops. Information about the micronutrient 
distribution can be obtained using in situ staining or spectroscopic techniques. Staining methods target specific metals, based on chemical reactions between the histological dye and the metal of interest. However, these reactions are subject to competitive exchange equilibrium with endogenous ligands and are usually considered liable to visualize only labile ions (McRae et al. 2009). Spectroscopic methods include proton-induced X-ray emission (PIXE) that targets the embryo region (Mazzolini et al. 1985), scanning and transmission electron microscopy (STEM) in combination with energy dispersive X-ray microanalysis (EDX) that focuses on aleurone and scutellum cells to provide subcellular information (Ockenden et al. 2004; Lombi et al. 2010), nanosecondary ion mass spectrometry (nano-SIMS) that visualizes the subcellular distribution but is limited to regions of only a few $\mu \mathrm{m}^{2}$ (Moore et al. 2010), and the X-ray fluorescence (XRF) method that provides elemental maps for various elements in whole grain sections (Lombi et al. 2009; Takahashi et al. 2009). The nondestructive $\mu$-XRF technique permits a three-dimensional reconstruction of accumulation patterns and can also distinguish between ionic valencies, critical for accumulation of toxic forms of various ions (Scheckel et al. 2007). More recently, Ryan et al. (2010) developed a large energy-dispersive detector array called, Maia, to capture intricate detail in natural material, together with faster acquisition and improved counting statistics in elemental imaging. A 96-detector prototype demonstrated the capacity of the system for real-time deconvolution of complex spectral data using an embedded implementation of the dynamic analysis method that acquires highly detailed images of up to $77 \mathrm{M}$ pixels spanning large areas of complex material sample sections. An excellent review by Lombi et al. (2010) focuses on the most recent status of in situ techniques to visualize spatial distributions and assess the speciation of metals and metalloids. Sample preparation probably constitutes the most critical step and is method (and to some extent also species-) dependent. The above-mentioned techniques differ in terms of resolution and sensitivity, depth of analysis, and in their capacity to provide mass resolution or molecular information.

Detailed knowledge of the distribution of macro- and micronutrients provides indications on possible ligands controlling the bioavailability of certain elements, such as Fe and $\mathrm{Zn}$, and this information may also be useful to minimize the losses during milling/polishing. The wheat seed at maturity consists of an outer layer of maternal origin comprising a testa derived from the integuments, the pericarp, and awns, while the central tissues consist of an embryo and endosperm, respectively, derived from single and double fertilization events. The outermost 
layer of the endosperm is differentiated into an aleurone. The endosperm is filled with starch and storage proteins, while the aleurone and the embryo accumulate a range of nutritional reserves such as minerals, carbohydrates, fats, and proteins, including enzymes. Mazzolini et al. (1985) found high concentrations of iron in wheat seed aleurone and the scutellum layer of the embryo and low concentrations in the endosperm. Barley endosperm and aleurone in contrast, together contain $\sim 70 \%$ of the total $\mathrm{Fe}$, but only $7 \%-8 \%$ in the embryo (Duffus and Rosie 1976). Zinc in wheat is predominantly located in the embryo and aleurone parts of the seed (Ozturk et al. 2006). The Zn concentration in seeds is particularly high during early seed development (i.e., at milking stage); thereafter, its concentration gradually declines until maturity. Using ear culture system (Singh and Jenner 1983; Sharma et al. 1995), the stable zinc isotope ${ }^{70} \mathrm{Zn}$, and laser ablation-inductively coupled plasma-mass spectrometry (LA-ICP-MS) technique, Wang et al. (2011) detected preferential localization of $\mathrm{Zn}$ in the aleurone and embryo, as also observed by Ozturk et al. (2006), but found a gradient of ${ }^{70} \mathrm{Zn}$ concentration between crease vascular tissue, aleurone layer, and endosperm, demonstrating that $\mathrm{Zn}$ is distributed within the seed through the crease phloem. This suggests that two barriers of Zn transport into wheat seeds may exist: between the stem tissue rachis and the seed, and between the maternal and filial tissues in the seed. Likewise, large gradients in the distribution of micronutrients were reported for both within and between different tissues of barley seed, with gradients especially evident in the embryo and the scutellum regions (Lombi et al. 2011). Moreover, the ventral and dorsal part of the barley seeds also showed significant differences in element distribution. Furthermore, the speciation analysis of barley seed tissues using SEC-ICP-MS and IP-ICP-MS techniques revealed highest concentrations of $\mathrm{Zn}, \mathrm{Fe}, \mathrm{S}$, and $\mathrm{P}$ in the bran and embryo fractions (Persson et al. 2009). Analysis of the embryo further revealed differences in speciation of $\mathrm{Fe}$ and $\mathrm{Zn}$. The majority of the $\mathrm{Fe}$ coeluted with $\mathrm{P}$ as a species with the apparent mass of $12.3 \mathrm{kDa}$, whereas the majority of $\mathrm{Zn}$ coeluted with $\mathrm{S}$ as a $3 \mathrm{kDa}$ species; devoid of any coeluting with $\mathrm{P}$. These results show that $\mathrm{Zn}$ appears to be bound mainly to peptides, whereas $\mathrm{Fe}$ is associated mainly with phytic acid.

Element-specific distribution patterns of micronutrients have been reported for rice seeds. Lombi et al. (2009) detected high concentrations of $\mathrm{Cu}, \mathrm{Fe}, \mathrm{Mn}$, and $\mathrm{Zn}$ in certain regions of the husk. However, the distribution of these nutrients varied considerably in other parts of the seed. For example, $\mathrm{Zn}$ in the central part of the embryo, which likely corresponds to the plumule; however, its concentration deceases 
gradually from the aleurone/pericarp and outer parts of the endosperm to the interior of the endosperm, while $\mathrm{Mn}$ and Fe very much localize in the aleurone/pericarp region with a sharp change in the concentration in the exterior parts of the endosperm. Mn is highly concentrated in the embryo but with a different pattern than observed for $\mathrm{Zn}$. The strong similarities between the distribution of $\mathrm{Fe}, \mathrm{Mn}$, and $\mathrm{P}$ and between $\mathrm{Zn}$ and $S$ may be indicative of the complexation mechanisms involved in rice seeds. Preliminary studies in pearl millet revealed greater concentration of seed minerals including $\mathrm{Fe}$ and $\mathrm{Zn}$ in the covering layers and the germ than in the endosperm portions, similar to most cereal seeds (Varriano-Marston and Hoseney 1980).

Common bean and soybean genotypes were reported to accumulate different proportion of total seed $\mathrm{Fe}$ in the seed coat, embryo, and cotyledons (Laszlo 1990; Moraghan and Grafton 2002; Moraghan et al. 2002; Moraghan 2004; Ariza-Nieto et al. 2007; Cvitanich et al. 2010, 2011), indicating that specific tissues relevant for Fe storage should be identified and their $\mathrm{Fe}$ loading mechanisms be investigated to exploit such variability toward developing seed iron-dense cultivars. Using PIXE assay to investigate Fe distribution in seed tissues of Phaseolus species, Cvitanich et al. $(2010,2011)$ concluded that (1) the distribution of Fe in seed depends on the species and genotype, (2) high concentrations of $\mathrm{Fe}$ accumulate in cells surrounding the provascular tissue, (3) the tissue in the proximity of the provascular bundles holds up to $500 \mu \mathrm{g} \mathrm{g}^{-1}$ $\mathrm{Fe}$, depending on genotypes, and (4) the largest proportion of seed Fe in Phaseolus species is stored in compounds and cell parts different from ferritin and starch vacuoles. These results indicate that more studies are needed to assess the patterns of micronutrient distribution in seeds, and that micronutrient distribution criteria should be integrated into the selection strategies for biofortification of staple crops.

In summary, CGIAR and national agricultural research systems (NARS) institutions hold large collection of germplasm, both cultivated and wild relatives' of cereal and legume crops. The core or mini-core collections available in these crops may be used to identify seed mineral-dense germplasm. The germplasm from regions deficient in soil micronutrients should receive priority for evaluation as such germplasm are expected to develop inherent adaptation mechanisms that favor enhanced nutrient acquisition, transport, distribution, and relocation in plants/seeds. Few germplasm lines with high seed-Fe and/or - Zn concentrations have been reported in common bean, maize, pearl millet, rice, sorghum, and wheat. Wild and weedy relatives of common bean and wheat have shown abundant variability for Fe and Zn. Mutants with moderate-to-high reduction in phytate are available in 
barley, common bean, maize, rice, soybean, and wheat. Genotypic differences in iron bioavailability have been reported in common bean, maize, rice, and wheat, which should be further explored. Several methods are available with high precision to map elements distribution, which may be used to identify barriers to Fe and $\mathrm{Zn}$ accumulation in the seed.

\section{EXPLOITING NATURAL GENETIC VARIATION TO BREED FOR SEED MINERAL-DENSE CULTIVARS}

\section{A. Fixing the Biologically Attainable Target to Breed for Seed Mineral-Dense Crops}

Several factors must be taken into consideration when setting the target levels for enhancing the nutritional status of food crops by breeding. These include (1) mapping the human populations with micronutrient deficiency, (2) food habits of those suffering from micronutrient malnutrition, (3) the major staple crops grown in micronutrient-deficient regions and their nutrient profiles, (4) the recommended micronutrient requirement vis-à-vis daily nutrient intake, (5) the genetic variation for micronutrients in germplasm pools and cultivars/hybrids produced in the region or of possible production there, and (6) the bioavailability, bioconversion, and bioaccessibility of the micronutrients in the crop or combination of crops consumed in the diet (Nestel et al. 2006; Ortiz-Monasterio et al. 2007; Pfeiffer and McClafferty 2007; Bouis and Welch 2010). The target set for crop biofortification for one nutrient may not be the same for a different micronutrient, and may further differ from one country or region to another. The baseline data of daily intake of minerals may vary as detected for iron concentration in India. The intake of iron in India is less than $50 \%$ of the recommended dietary

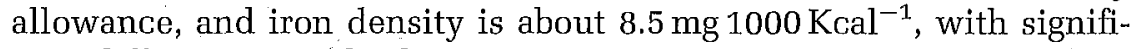
cant differences in absolute amounts among regions. Diets in Indian state of Andhra Pradesh with rice as staple have lowest iron (7 mg 1000 $\mathrm{Kcal}^{-1}$ ), while diets in Gujarat and Madhya Pradesh with pearl millet as the staple have the highest iron intake (16 mg $1000 \mathrm{Kcal}^{-1}$ ) (Nair and Iyengar 2009). Likewise, several target regions for cróp biofortification ( $\mathrm{Fe}, \mathrm{Zn}$, and provitamin A) interventions have been identified in Latin America and the Caribbean (Zapata-Caldas et al. 2009). For example, interventions in northern Colombia appear promising for all crops, while sites for bean biofortification are widely scattered throughout the country. The most promising sites in Nicaragua are found in the 
center-north region, while candidate sites for biofortification in Bolivia are found in the central part of the country and in the Andes Mountains. Poverty levels indicated that northeast Brazil is the most important region for biofortification in that part of South America.

Variations in the conversion factor of provitamin $A(\beta$-carotene) to vitamin A (retinol) in food crops should also need to be considered when defining breeding targets for $\beta$-carotene (Tang 2010). Significant genetic variation for seed-Fe and -Zn concentrations has been reported for cereal and legume crops, with some genotypes having more bioavailable seed micronutrients than others (see Section V.A). Most modern cultivars/hybrids have lower micronutrients per se than those reported in germplasm pools of a given species (Graham et al. 1999; Frossard et al. 2000). The adverse effects of processing, storage, and cooking on nutrient concentrations losses are known (see Section III.B.2). In addition, there are certain elements present in the seed that either act as enhancers (i.e., ascorbic acid) or inhibitors (i.e., phytase) of micronutrient uptake and absorption (see Section III.B.1).

All these variables need to be factored in when setting the breeding targets for improved nutritional quality of food crops. HarvestPlus has set the tentative breeding targets for improving the micronutrient density of several food crops. For example, the tentative targets to increase seed-Fe concentration of rice, wheat, pearl millet, common beans, maize, cassava, and sweet potato are $15,59,88,107,60,45$, and $85 \mu g^{-1}$ on a dry weight basis, respectively, while those for $\mathrm{Zn}$ are fixed at 28, 38, 66, 56, 38, 34, and $70 \mu \mathrm{gg}^{-1}$ (Bouis and Welch 2010).

For provitamin $A$, the targets set are $17,17,23,34,17,48$, and $91 \mu \mathrm{gg}^{-1}$ for rice, wheat, pearl millet, common bean, maize, cassava, and sweet potato, respectively (Bouis and Welch 2010). Such an approach can be applied to define the target levels for other micronutrients as well. However, targets should be dynamic depending on the severity of the micronutrient deficiency and the progress realized through breeding for developing mineral-dense cultivars/hybrids.

\section{B. Genotype $\times$ Environment Interaction and Relationships Between Seed Minerals and Agronomic Traits}

Knowledge of the effects of $G$, environments (E), and genotype $\times$ environment interaction (GEI) is important for developing nutritionally enhanced crop cultivars. Like yield and yield attributing traits, seed$\mathrm{Fe}$ and $-\mathrm{Zn}$ concentrations in common bean, maize, rice, and wheat are influenced by location (or E), G, and GEI (Table 3.2), with location effects generally much larger than those of either $\mathrm{G}$ or GEI effects: The 


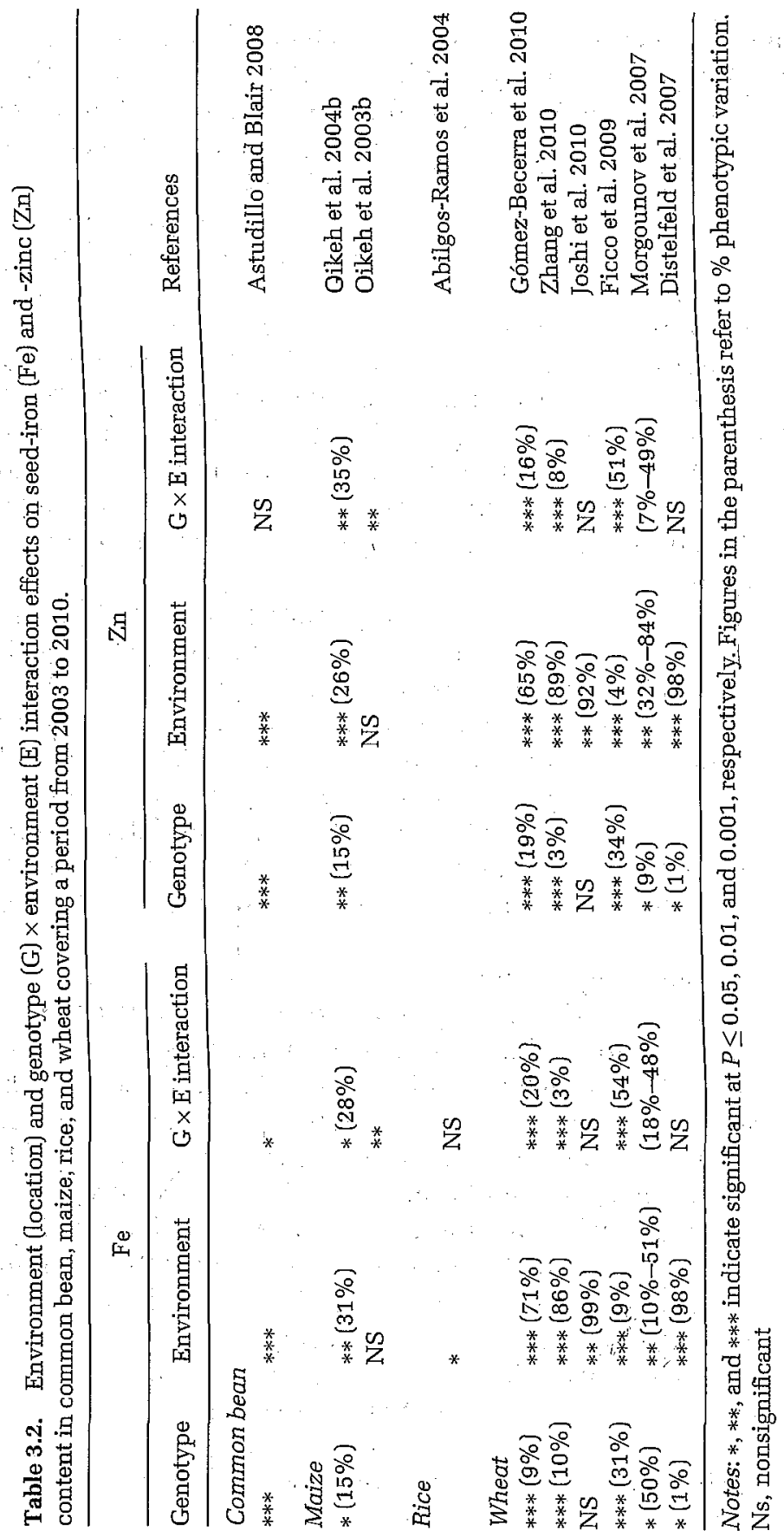


growing environments had no effect on bioavailable Fe in maize (Oikeh et al. 2004a); however, Pixley et al. (2011a) detected larger E than GEI for Fe bioavailability in maize. It is therefore suggested that responses of cultivars to different production environments need to be well understood to improve the probability of predicting and identifying cultivars that are not only high in seed-Fe and/or -Zn concentrations but also these micronutrients are more bioavailable to absorption (Briat and Lobreaux 1997; Pixley et al. 2011a).

The environmental variables such as $\mathrm{pH}$, temperature, solar radiation, precipitation, organic matter, and soil texture have the potential to influence nutrient concentration (Tisdale and Nelson 1975; Römheld and Marschner 1986; Cabuslay et al. 2003; Abilgos-Ramos et al. 2004; Joshi et al. 2010) and must be taken into consideration while explaining the variation for seed micronutrients in germplasm or when assessing the nutritional quality of staple food crops grown in diverse agroecological conditions.

Character association between seed mineral concentrations may indicate the existence of one or more common genetic-physiological mechanisms involved in mineral uptake by the root system, translocation, and redistribution within the plant tissues, remobilization to the seed, and accumulation in the developing seed (Chatzav et al. 2010). Both mineral concentration (amount per unit weight, i.e., $\mathrm{mg} \mathrm{kg}^{-1}$ ) and mineral content (amount per seed, i.e., $\mu$ seed $^{-1}$ ) are positively correlated (Cakmak et al. 2004; Hacisalihoglu et al. 2005; Stangoulis et al. 2007) and either can be used to estimate the quantity of the minerals in the seeds (see Section V.A).

An understanding of the nature of association between different minerals and also with seed yield and seed weight (100 or 1,000seed weight) should facilitate the selection of mineral-dense progenies in breeding. The published evidence suggests that seed-Fe and $-\mathrm{Zn}$ concentrations, in most cases, are highly significant and positively correlated in common bean, pearl millet, rice, and wheat (Table 3.3), which suggests that genes for $\mathrm{Fe}$ and $\mathrm{Zn}$ accumulation cosegregate or are pleiotropic. Such relationships could be exploited toward selecting progenies with high seed minerals in the segregating populations. Further studies revealed that Fe and $\mathrm{Zn}$ in the flag leaves of Aegilops species are highly significant and positively correlated with seed Fe and Zn (Rawat et al. 2009a,b); however, such relationships were not found in common bean (Tryphone and Nchimbi-Msolla 2010). More studies are needed to elucidate these relationships prior to using flag leaf for early selection of plants with potentially high seed $\mathrm{Fe}$ and $\mathrm{Zn}$ in breeding programs. 


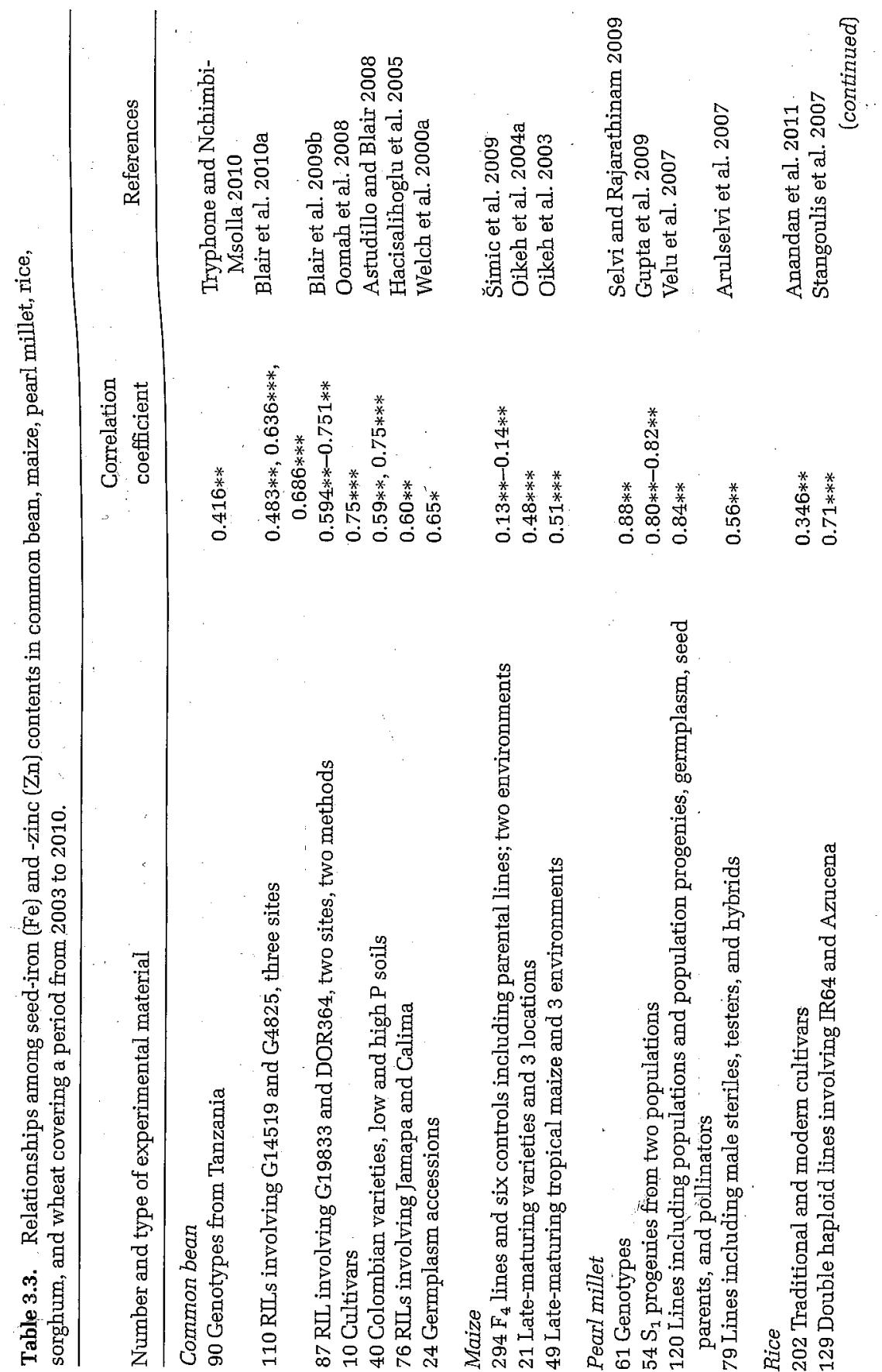




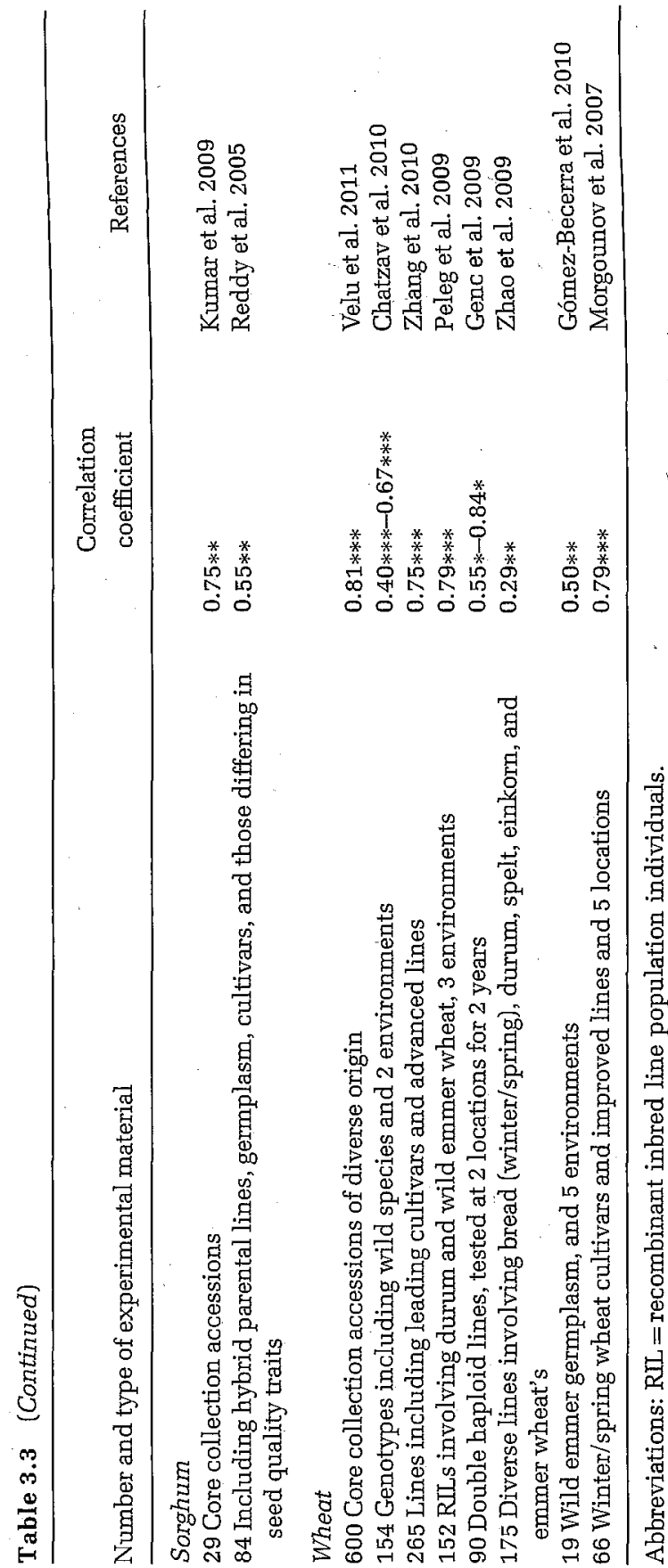


Highly significant and positive correlations (0.82-0.99) between seed $\mathrm{P}$ and phytate have been reported for common bean, pearl millet, and rice (Lolas and Markakis 1975; Stangoulis et al. 2007; Selvi and Rajarathinam 2009), while a low but positive and significant association was found for seed phytate with $\mathrm{Fe}$ and $\mathrm{Zn}$ in common bean (Cichy et al. 2009). Further, several studies in wheat and common bean revealed a moderate but positive and significant association of seed $\mathrm{P}$ with $\mathrm{Fe}$ (0.42-0.55) and Zn (0.46-0.63) (Gelin et al. 2007; Peleg et al. 2009; Zhao et al. 2009; Zhang et al. 2010). The implications of the above are that while it should be possible to breed for high seed-Fe and -Zn concentrations with reduced phytate concentration in pearl millet, this may not be possible in wheat or common bean.

In yet-another issue of correlations and micronutrient versus macronutrient concentrations, seed yield is significantly and positively associated with seed weight in cereals and legumes (Upadhyaya et al. 2002; García del Moral et al. 2003; Upadhyaya 2003; Maman et al. 2004; Morgounov et al. 2007). However, it is either not associated with seedFe and -Zn concentrations in pearl millet or shows a low but significant negative association in wheat (Morgounov et al. 2007; Peleg et al. 2009; Zhao et al. 2009) and positive association in common bean (Gelin et al. 2007). A negative association may pose problems for breeding of seed mineral-dense cultivars with high seed yield per se. Seed weight in pearl millet is highly significant and positively associated with seed $\mathrm{Fe}$ $(r=0.80)$ and $\mathrm{Zn}(r=0.85)$ (Velu et al. 2007), while it is significant and positively correlated $(r=0.61)$ with $\mathrm{Fe}$ in common bean (Gelin et al. 2007).

Another question is whether there are any relationships between seed-Fe (or - Zn) concentration and bioavailability. Limited studies on seed-Fe and -Zn concentration and bioavailability in maize, rice, and wheat revealed no such associations (Glahn et al. 2002; Oikeh et al. 2003a,b,2004a), indicating that it is possible to significantly increase both concentration and bioavailability of either Fe or Zn in the seed by breeding and selection.

\section{Quantitative Trait Loci (QTL) Associated with Seed Iron, Zinc, and Phytate Concentrations}

Genomics science since the 1990s has made phenomenal advances toward developing a large number of molecular markers and genetic linkage maps allowing the mapping and/or cloning of QTL and identification of candidate gene(s) associated with agriculturally beneficial traits, which can lead marker-assisted selection (MAS) 
for desirable genes in crop breeding (Dwivedi et al. 2007; Collard and Mackill 2008). Automation of high-throughput assays including next generation sequencing technologies and associated data mining tools provide breeders/molecular biologists opportunities to handle and interpret large data sets (Varshney et al. 2009; Feuillet et al. 2010). Furthermore, the genomes of agriculturally important crops such as maize, rice, sorghum, and soybean have been sequenced (IRGSP 2005; Paterson et al. 2009; Schanable et al. 2009; Schmutz et al. 2010), while several projects under way to sequence genomes of many other agriculturally important food crops (Feuillet et al. 2010). The deoxyribonucleic acid (DNA) sequence variants across species or among strains within a species may be used as new genetic tools for developing markers and subsequently crop cultivars with specific characteristics.

The crops included in this review have abundant genetic resources to dissect population structure and diversity in germplasm collections to identify genetically diverse germplasm with beneficial traits. However, only recently have DNA marker-based technologies been used to identify QTL associated with increased seed-Fe and -Zn concentrations in barley, common bean, pearl millet, rice, and wheat, revealing many QTL with varying effects; some with major phenotypic variation while many others with minor effects (Table 3.4). For example, in common bean a QTL on linkage group (LG) b09 was found for Zn by Gelin et al. (2007), while QTL found by Cichy et al. (2009) on LG b01 (near the fin gene) accounted for $34 \%$ of variation for seed-Fe and -Zn concentrations and also overlapped with a major QTL (19\% variation) for increased seed phosphorus (P) concentration. Finally, other QTL on LG b06 accounted for 36\% variation for seed Fe and $39 \%$ variation for seed $\mathrm{Zn}$ that same study (Cichy et al. 2009). This latter QTL was linked with a QTL found for Mesoamerican beans by Blair et al. (2010c). Further studies by Blair et al. (2009b, 2010b) in both inter- and intragene pool populations, respectively, found specific major and minor QTL for Fe and Zn concentrations with the former type mainly on LG b11. Therefore, at least four major QTL have been identified in common bean affecting micronutrient concentration depending on the gene pool and genetic background of the material tested.

In other crops, meanwhile, a QTL for seed-Zn on chromosome 7A mapped at Xcfd31-Xcfa2049 explained 19\% variation in wheat (Tiwari et al. 2009). In rice, a seed-Zn QTL mapped at RM235-RM17 on chromosome 12 accounted for $13 \%$ variation and colocated with seed-Fe QTL that mapped at RM270-RM17 and accounted $14 \%$ 


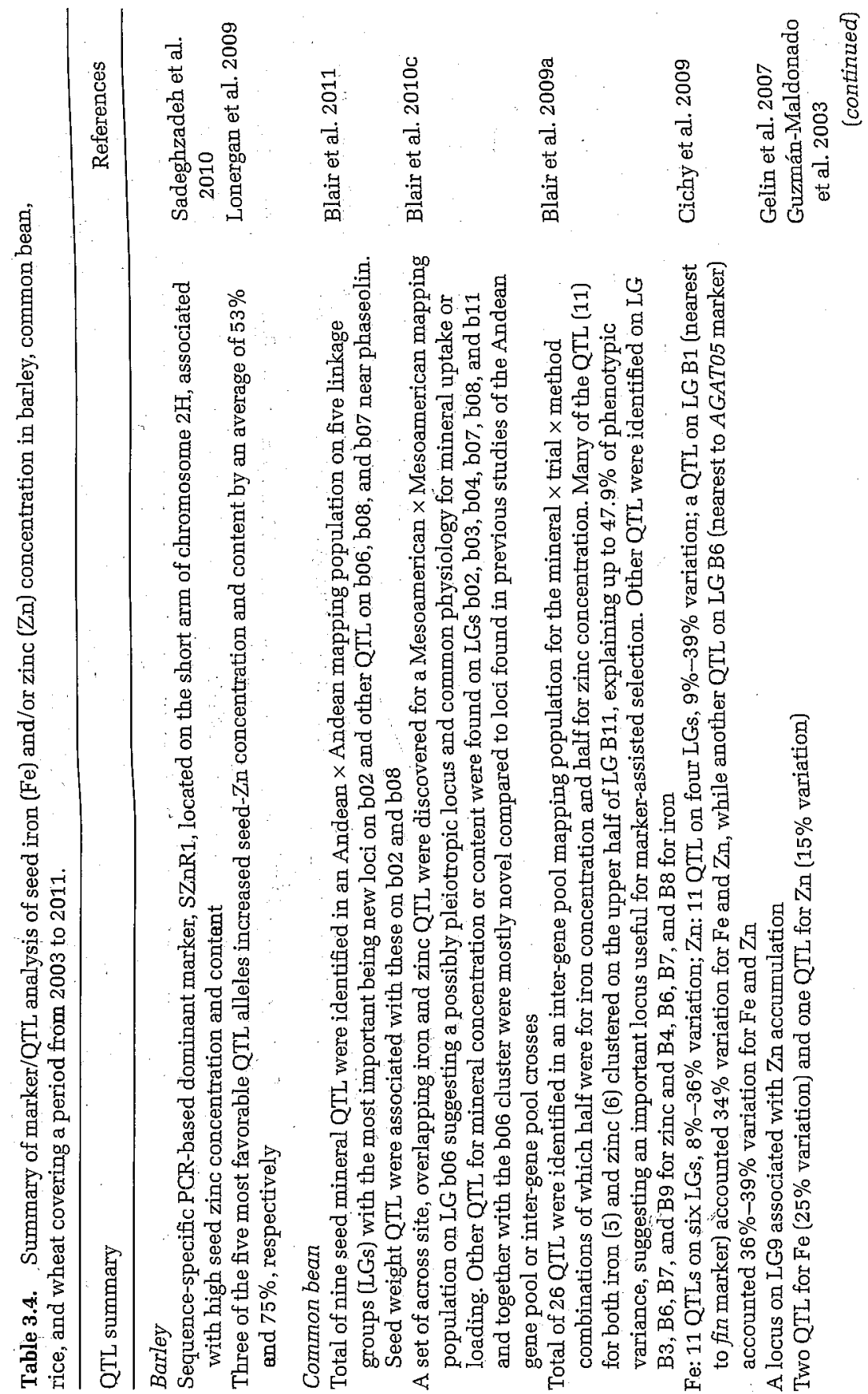




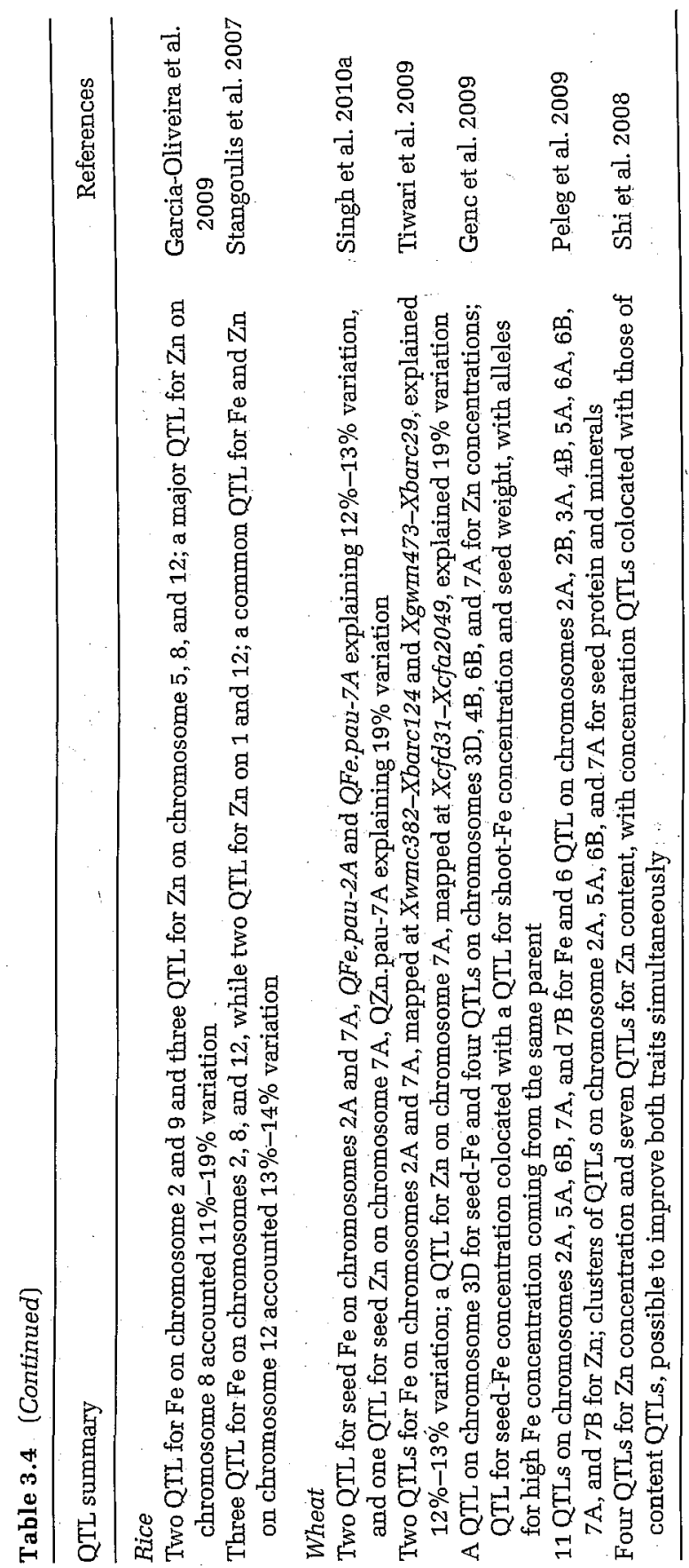


phenotypic variation (Stangoulis et al. 2007). In barley, specific markers have been developed for a high zinc QTL on chromosome 2H (Sadeghzadeh et al. 2010), while other QTLs have been identified by Lonergan et al. (2009).

Iron reductases are members of the protein super-family of flavocytochromes and function in roots to convert Fe from a plant unavailable form (ferric, $\mathrm{Fe}^{3+}$ ) to an available form (ferrous, $\mathrm{Fe}^{2+}$ ) that can be readily absorbed (Grusak 1995). IRA is known to vary with plant growth conditions (e.g., soil pH and available iron concentration) (Grusak 2000). Common bean genotypes with high seed-Fe showed high IRA than those with low seed-Fe, suggesting a link between root uptake and seed loading of $\mathrm{Fe}$ in common bean (Grusak 1994, 2000, 2002). More recently, Blair et al. (2010b) reported a single major QTL for IRA under Fe-limited conditions ( $1 \mu \mathrm{M}$ ) on LG b02, and another major QTL under Fe-sufficient conditions $(15 \mu \mathrm{M})$ on $\mathrm{LG}$ b11 that was associated with several QTL for seed Fe in common bean. Thus, the QTL for IRA under Fe-limited conditions may be useful in environments where beans are grown in alkaline soils, while the QTL for IRA under Fe-sufficient conditions may be useful for selecting for enhanced seed nutritional quality (Blair et al. 2010b).

Wild emmer wheat germplasm harbors a rich allelic diversity, including for seed minerals (Xie and Nevo 2008). A major locus, Gpc-B1 (a 250-kb locus) mapped as a simple Mendelian locus (Distelfeld et al. $2006)$, associated with increased seed-protein (38\%), -Fe (18\%), and - Zn $(12 \%)$ concentrations from wild emmer wheat germplasm (Triticum dicoccoides), encodes a NAC transcription factor (NAM-B1) that accelerates senescence and increases nutrient remobilization from leaves to developing seeds (Uauy et al. 2006; Distelfeld et al. 2007). Triticum turgidum is another useful wild emmer germplasm for improving seed mineral concentration in wheat. Peleg et al. (2009) mapped 82 QTL for 10 seed minerals (LOD score range of 3-17), with most of the positive alleles contributed by wild emmer accession, G18-16, and many QTL for the same trait mapped to homoeologous positions, reflecting synteny between the $\mathrm{A}$ and $\mathrm{B}$ genomes. TtNAM-B1 affecting seed-protein, -Fe, and -Zn originating from wild emmer wheat has been cloned (Distelfeld and Fahima 2007). Furthermore, Singh et al. (2010a) identified two QTL (QFe.pau-2A and QFe.pau-7A) for Fe and a QTL (QZn.pau-7A) for $\mathrm{Zn}$, which they transferred into interspecific progenies involving Aegilops kotschyi and Aegilops peregrine, both UUSS genome species. Such progenies showed $60 \%-136 \%$ enhanced seed-Fe and -Zn concentrations and $50 \%-120 \%$ increased $\mathrm{Fe}$ and Zn contents per seed as compared to the control cultivar that was introgressed with these 
QTL (Tiwari et al. 2010). The profiling of introgression using simple sequence repeats (SSRs), genomic in situ hybridization (GISH), and fluorescent in situ hybridization (FISH) analysis further confirmed the introgression of chromosome 2S, 2U, 7S, and 7U into these progenies (Singh et al. 2010a). More recently, genetic mapping identified five putative QTL for seed-Fe density and two QTL for seed-Zn density in pearl millet (Kumar et al. 2010b).

A number of QTL for seed phosphorus (P) and/or phytate concentrations have been reported in common bean, rice, sorghum, soybean, and wheat (Table 3.5), some with either major effects or colocated with QTL affecting seed-Fe or -Zn concentration. For example, a major QTL for $P$ (19\% variation) on b01 colocated with QTL accounting 34\% variation each for seed Fe and $\mathrm{Zn}$ in common bean (Cichy et al. 2009). Furthermore, QTL for seed P or phytate concentration or content related to seed weight QTL on LGs b06, b07, and b10 (Blair et al. 2009c) or genes coding for candidate enzymes involved in phytic acid synthesis pathway and markers associated with each gene (Fileppi et al. 2010) have been mapped in common bean. In rice, the candidate gene for low-phytate mutant alleles and markers (LPA1_CAPS for Ipa1-1 and LPA1_InDel for lpa1-2) showed complete cosegregation with mutant phenotypes (Zhao et al. 2008). Furthermore, the two QTL for seed-Zn concentration on chromosomes 4A and 4D colocated with QTL for P concentration, while four QTL for seed-Zn content on chromosome $2 \mathrm{D}, 3 \mathrm{~A}$, and $4 \mathrm{~A}$ colocated with the QTL for P content, reflecting positive correlation between the seed-Zn and -P concentrations (see Section VI.B), which may provide opportunities for simultaneous improvement in seed-P and -Zn density in wheat (Shi et al. 2008).

Two QTL mapped onto LGs L and N control low phytate in soybean line, GX 1834 (Walker et al. 2006; Gao et al. 2008). Further, Saghai-Maroof et al. (2009) mapped and sequenced a putative multidrug resistanceassociated protein (MRP) gene on LG $N$ that contributes to low-phytate phenotype in CX 1834. This A to T mutation provided a single nucleotide polymorphism (SNP) marker for introgressing the low-phytate QTL from CX 1834 into desired breeding lines (Saghai-Maroof et al. 2009).

More recently, genes coding for candidate enzymes involved in the phytic acid pathway have been mapped and identified markers associated with each gene ( $P_{V M} M I P S s, P_{V M I P S V}$ PVIMP, PVMIK, PVIPK2, PVITPKó, PVITPK $\beta$, PVIPK1), which may represent a useful resource to select genetic variants with low-phytate trait in common bean (Fileppi et al. 2010). Furthermore, González et al. (2010). discovered how phytate is produced in plants, by solving the structure of the protein $\operatorname{InsP}_{5}$ 2-Kinase $\left(\mathrm{IP}_{5} 2-\mathrm{K}\right.$ ), a distant member of the IPK family, 


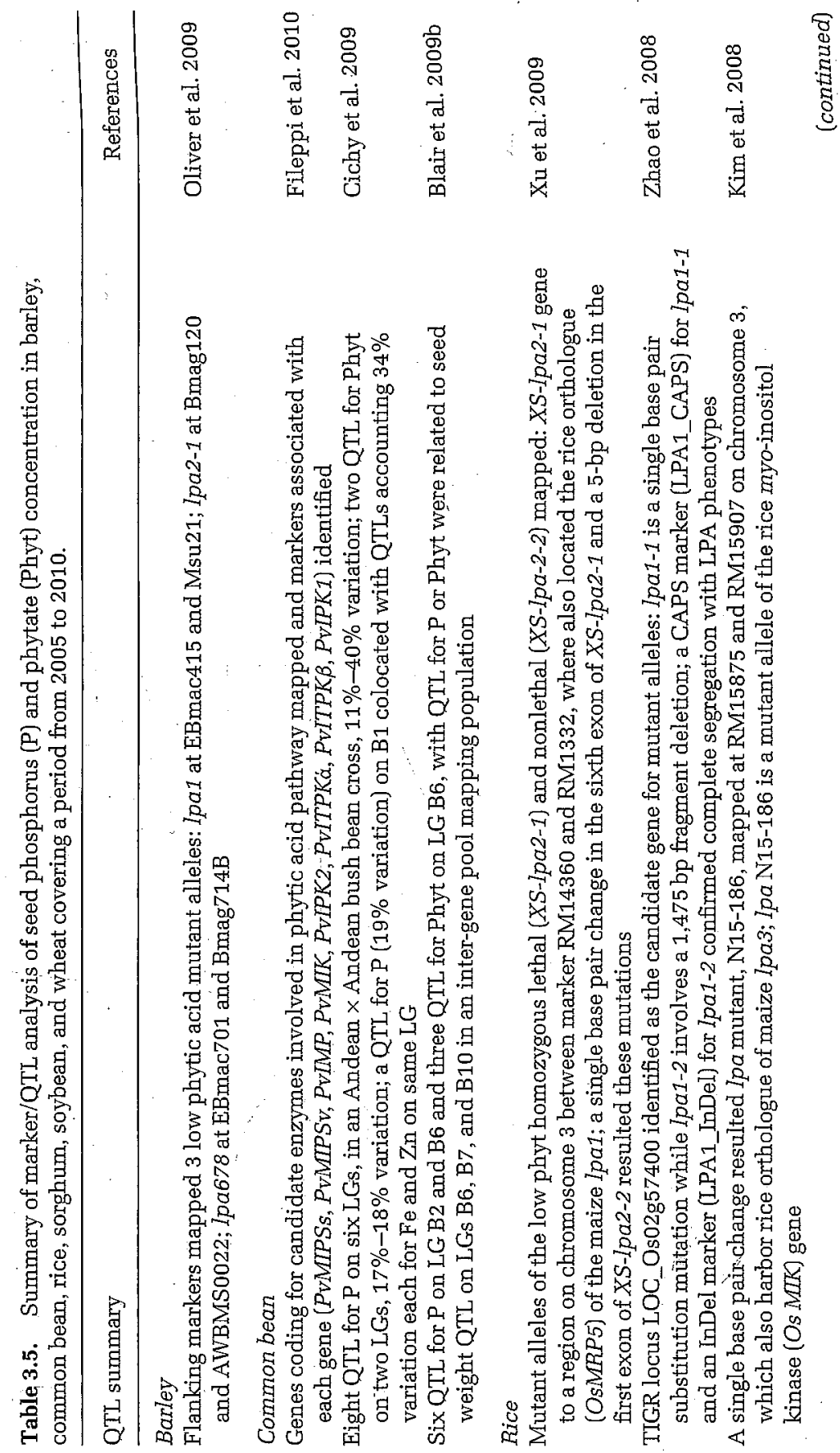




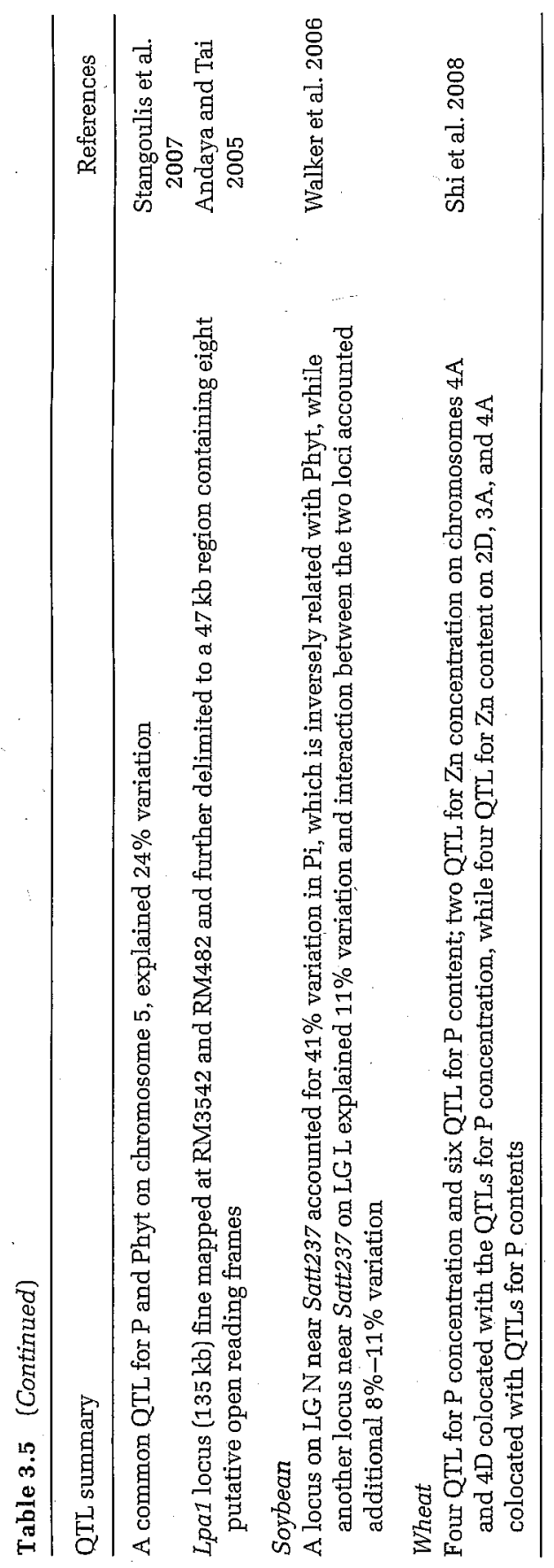


which represents a key point in the metabolism of highly phosphorylated inositols, and could be a valuable tool in plant physiology to design low-phytate crops.

\section{QTL Mapping, Cloning, and Introgression of $\beta$-Carotene into Adapted Germplasm}

The natural plant pigments, termed as carotenoids, which are fat soluble, are important source of vitamin A and antioxidants. The two groups of carotenoids are provitamin A and nonprovitamin A carotenoids. The former includes three carotenoids, namely $\beta$-carotene, $\beta$-cryptoxanthin, and $\alpha$-carotene, all precursor for vitamin $A$, while the latter consists of lutein and zeaxanthin, which act as antioxidants. Of all the carotenoids, $\beta$-carotene is the most widely distributed in plants and the one most efficiently converted to vitamin $A$. The consumption of carotenoid-rich foods is associated with reduced risks of developing cancer and cardiovascular diseases, enhanced immune responses, improved vision, and prevention of night blindness as well the maintenance of healthy skin and gastrointestinal or respiratory systems. Carotenoids in plants play a crucial role in photosynthesis, membrane stability, growth, and development (Menkir et al. 2008).

Unlike other agronomically important traits, only limited germplasm sets have been assessed for $\beta$-carotene, predominantly in chickpea, maize, pearl millet, sorghum, and wheat (Table 3.6), with some lines accumulating $\beta$-carotene as high as $7.6 \mu^{-1} g^{-1}$ in pearl millet (Hash et al. 1997) and $15.6 \mu \mathrm{g} \mathrm{g}^{-1}$ in maize (Harjes et al. 2008; Menkir et al. 2008). Genotypic differences for $\beta$-carotene have also been reported in rice: some germplasm had shown $\beta$-carotene in unpolished seeds while others having no $\beta$-carotene in unpolished seeds. The rice germplasm with $\beta$-carotene in unpolished seeds include Amarillo Cuba, Dudemasino, Sirendah Kuning, Bongkitan, Calibo, Khao Dawk Mali 105, and Klemas (Tan et al. 2005). Furthermore, Kandlakunta et al. (2008) in a comprehensive study involving major cereals and legumes and commonly consumed vegetables detected high $\beta$-carotene in chickpea $\left(15.7 \mu \mathrm{g} \mathrm{g}^{-1}\right)$, green gram $\left(12.8 \mu \mathrm{g} \mathrm{g}^{-1}\right)$, red gram $\left(12.4 \mu \mathrm{g} \mathrm{g}^{-1}\right)$, and maize $\left(17.1 \mu \mathrm{g} \mathrm{g}^{-1}\right)$, while among vegetables, they detected high $\beta$-carotene in yellow pumpkin $\left(118 \mu \mathrm{gg}^{-1}\right)$, green chillies $\left(102 \mu \mathrm{gg}^{-1}\right)$, field beans $\left(55.4 \mu \mathrm{g} \mathrm{g}^{-1}\right)$, French bean $\left(39.3 \mu \mathrm{g} \mathrm{g}^{-1}\right)$, ridge gourd $\left(32.4 \mu \mathrm{g} \mathrm{g}^{-1}\right)$, green beans $\left(23.9 \mu \mathrm{gg}^{-1}\right)$, and brinjal $\left(16.9 \mu \mathrm{gg}^{-1}\right)$.

Maize is an important source of provitamin A ( $\beta$-carotene, $\alpha$-carotene, and $\beta$-cryptoxanthin) and the nonprovitamin $A$ including lutein and zeaxanthin. Most of the yellow maize grains consumed worldwide have 


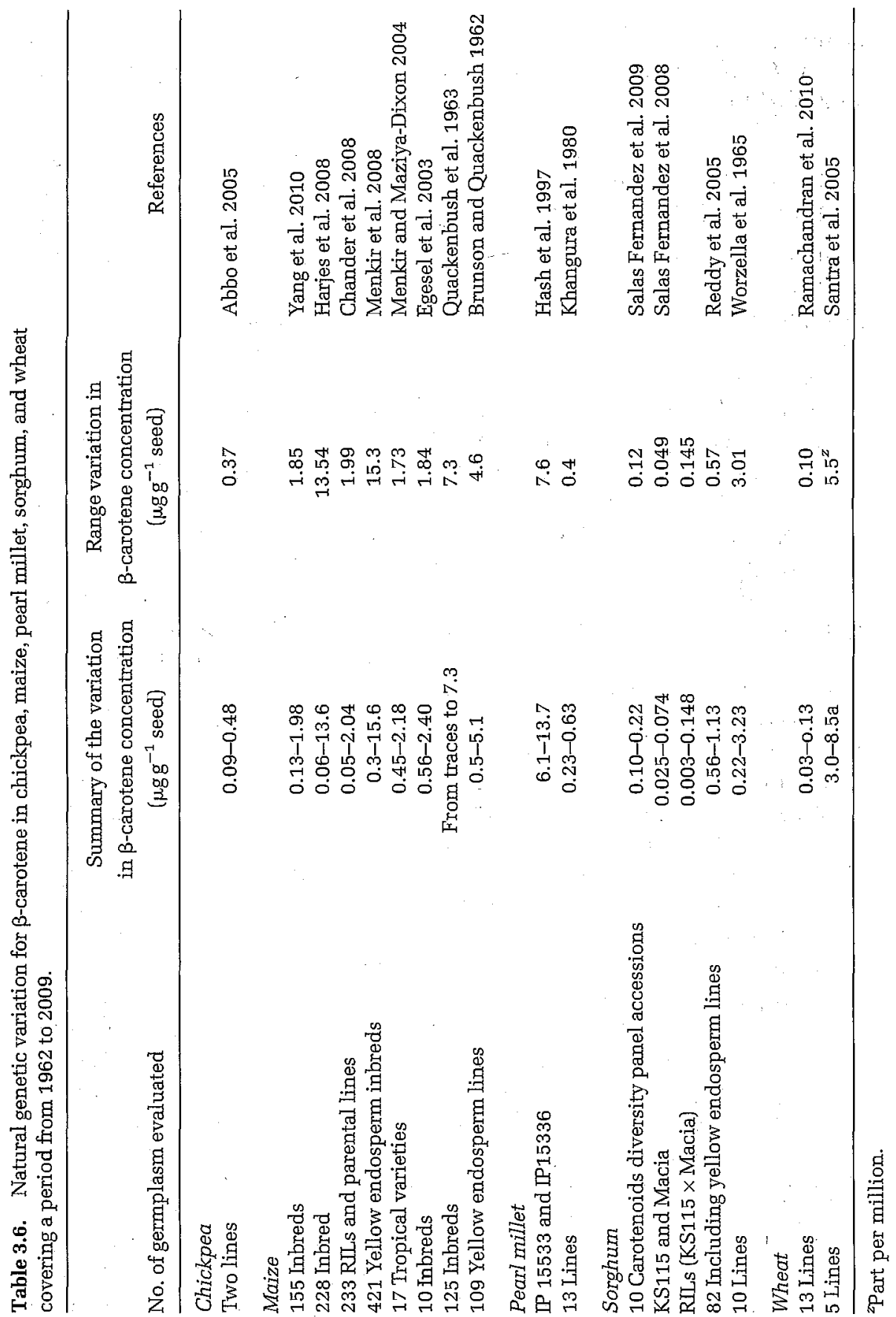


only $0.5-1.5 \mu \mathrm{gg}^{-1} \beta$-carotene. Maize has been extensively studied for molecular polymorphism, mapped QTL, chemical pathways, and genes involved in carotenoids biosynthesis, including $\beta$-carotene (Table 3.7): Carotenes are intermediates in the carotenoids biosynthetic pathway and lycopene (the immediate precursor of provitamin A carotenes) represents a branch point in this pathway, which is further modified by lycopene $\beta$-cyclase and lycopene $\varepsilon$-cyclase (LCYE) enzymes that catalyze formation of terminal $\beta$ - and $\varepsilon$-rings, respectively, to form either $\beta$-carotene, $\alpha$-carotene, or $\beta$-cryptoxanthin. This pathway continues with hydroxylation of the carotenes that depletes the provitamin A pool by converting these compounds to nonprovitamin A xanthophylls. Pathway branching and hydroxylation are therefore key determinants in controlling vitamin A levels. Polymorphism at the LCYE locus in maize explained $58 \%$ of the variation in $\alpha$ - and $\beta$-carotene and a threefold difference in provitamin A compounds (Harjes et al. 2008), while a rare genetic variant, $\beta$ carotene hydroxylase 1 (crtRB1), increases $\beta$-carotene substantially in maize grains (Yan et al. 2010). Further, metabolite sorting of a germplasm collection identified 10 genetically diverse subsets representing biochemical extremes for maize kernel carotenoids and transcript profiling of this subset led to the discovery of the Hydroxylase 3 locus that coincidently mapped to a carotene QTL (Chander et al. 2008). The natural alleles at Hydroxylase 3 locus contribute $78 \%$ of variation and approximately 11-fold differences in $\beta$-carotene relative to $\beta$-cryptoxanthin and $36 \%$ of the variation and fourfold difference in absolute levels of $\beta$-carotene (Harjes et al. 2008). The reduction in: $H Y D 3$ transcripts leads to reduced conversion of $\beta$-carotene to downstream xanthophylls, causing $\beta$-carotene to accumulate (Vallabhaneni et al. 2009). Genetics tests such as the HYD3 assay (Vallabhaneni et al. 2009) together with the previously described $L C Y E$ assay (Harjes et al. 2008) may be used to select germplasm containing optimal $H Y D 3$ and $L C Y E$ alleles in breeding programs, which will lead to higher $\beta$-carotene levels in maize endosperm when both genes are highly expressed than either with optimal alleles of either gene alone. Furthermore, the experimental evidence from the association and linkage mapping reveals that $\operatorname{crtRB1}$ underlies a principal QTL associated with $\beta$-carotene concentration and conversion in maize kernels and $c r t R B 1$ alleles associated with reduced transcript expressions correlate well with higher $\beta$-carotene (Yan et al. 2010). The most favorable $\operatorname{crtRB} 1$ alleles that are rare in frequency and unique to temperate germplasm are being introgressed via inexpensive PCRmarker-assisted selection into tropical maize germplasm adapted to developing countries. A program at CIMMYT has already achieved 


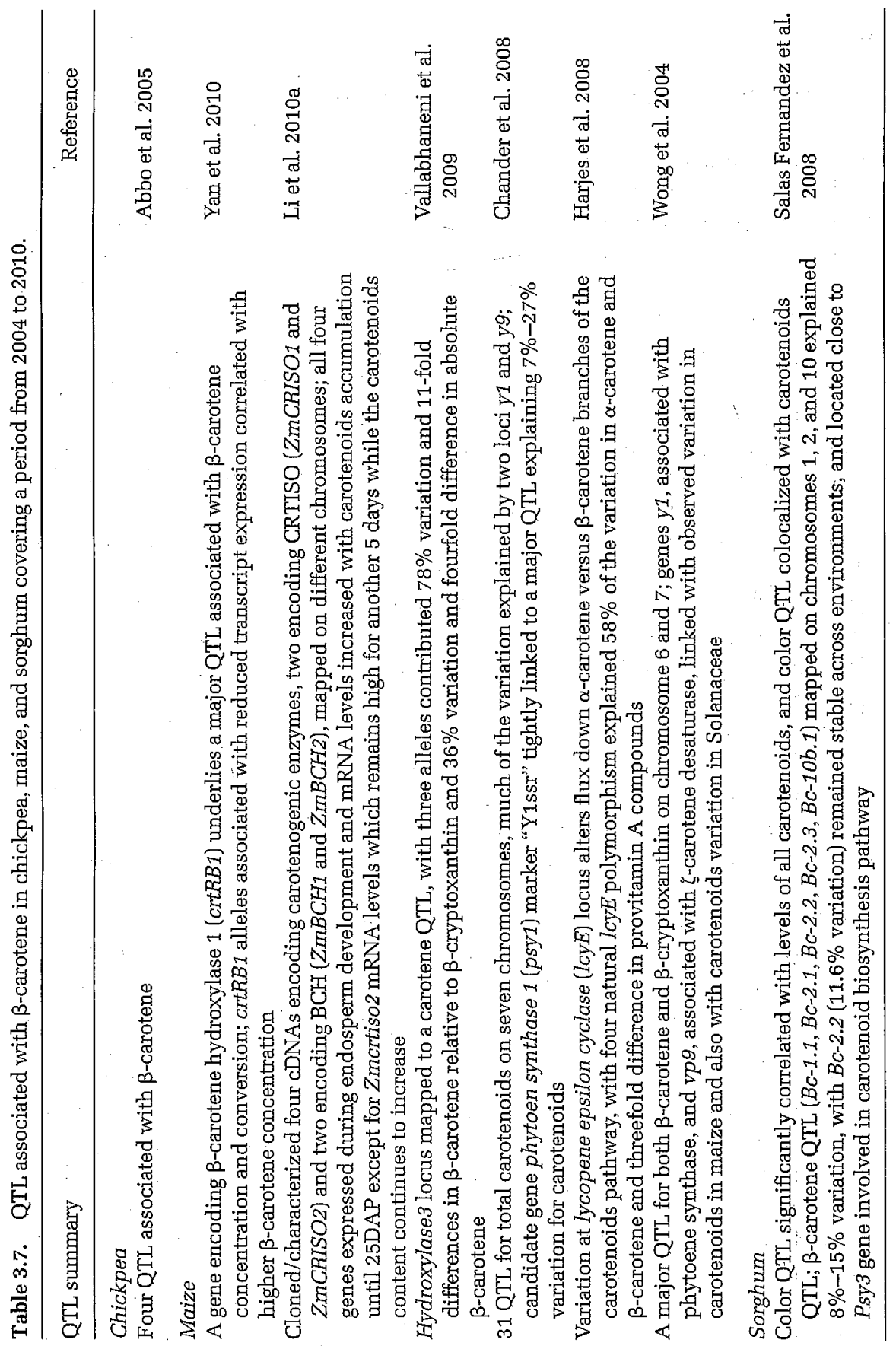


HarvestPlus provitamin A target concentrations by introgressing favorable $c r t R B 1$ and lcys alleles into tropical maize germplasm in a number of breeding lines, including a high $\beta$-carotene $\left(15 \mu \mathrm{gg}^{-1}\right)$ temperate hybrid, CI7 $\times$ DEexp that contains the most favorable crtRB1 alleles (Yan et al. 2010; Pixley et al. 2011b). Several hybrids with $\beta$-carotene concentration between 5 and $8 \mu \mathrm{gg}^{-1}$ and agronomically competitive with commercial hybrids were tested during summer 2009/2010 in Zambia and Zimbabwe, and the best hybrids will be further evaluated in Mexico, Zambia, and Zimbabwe prior to their release in these countries (Pixley et al. 2011b). Likewise, the International Institute of Tropical Agriculture (IITA), Ibadan, Nigeria, has introduced high $\beta$-carotene trait from temperate maize to tropical maize inbred lines, which ranged from $2.5 \mu \mathrm{g}^{-1}$ to $10.5 \mu \mathrm{g}^{-1}$, and hybrids involving some of these inbreds showed $25 \%-79 \%$ more provitamin A concentration than Oba Super II, a commercial yellow hybrids widely grown in Nigeria. The grain yield and agronomic traits of the best hybrids were comparable to those of Oba Super II (IITA annual report, 2009/2010; http:// annualreport.iita.org/ $? \mathrm{p}=481$ ). The University of Illinois, USA has also reported some of the high provitamin A lines of maize that include A 619, C 17, DE 3, and SC 55 (see supplementary Table 1, Yan et al. 2010). The researchers at Vivekananda Parvatiya Krishi Anusandhan Sansthan (VPKAS), Almora, India and other affiliated Indian Council of Agricultural Research (ICAR) institutions in India have identified few promising maize inbred lines (CM 136 and CM 138, 08 HPLET-03-8 and 08 HPLET-03-41, NAI 125, BAJIM-8-10 and SE 547), with total carotenoids ranging from 20.2 to $56.1 \mu \mathrm{gg}^{-1}$ seed and $\beta$-carotene from 10.6 to 14.9 $\mathrm{gg}^{-1}$ seed (P.K. Agrawal, VKPAS, pers. commun.).

The 'Golden Rice 2' genetic stocks have been used to introgress high $\beta$-carotene trait into several Asian rice cultivars, both japonica and indica types, and it is expected that the products from such introgressions will soon be available for national release in Bangladesh, India, Indonesia, and the Philippines (Barry 2011).

Sorghum landraces have shown significant variation for carotenoids, with lutein, zeaxanthin, and $\beta$-carotene the predominant carotenoids. Yellow-endosperm color QTL in sorghum colocalized with carotenoid QTL, with major $\beta$-carotene QTL $B c-2.2$ found close to Psy3 gene, which is significantly associated with $\beta$-carotene concentration and endosperm color (Salas Fernandez et al. 2008). Furthermore, Salas Fernandez et al. (2009) reported that 164 yellow endosperm landraces from Niger and Nigeria clustered separately from the genotypes in a $6 \widehat{8}$ individual diversity panel (Casa et al. 2008) with accessions differing in geographic origin and carotenoids content, which may provide 


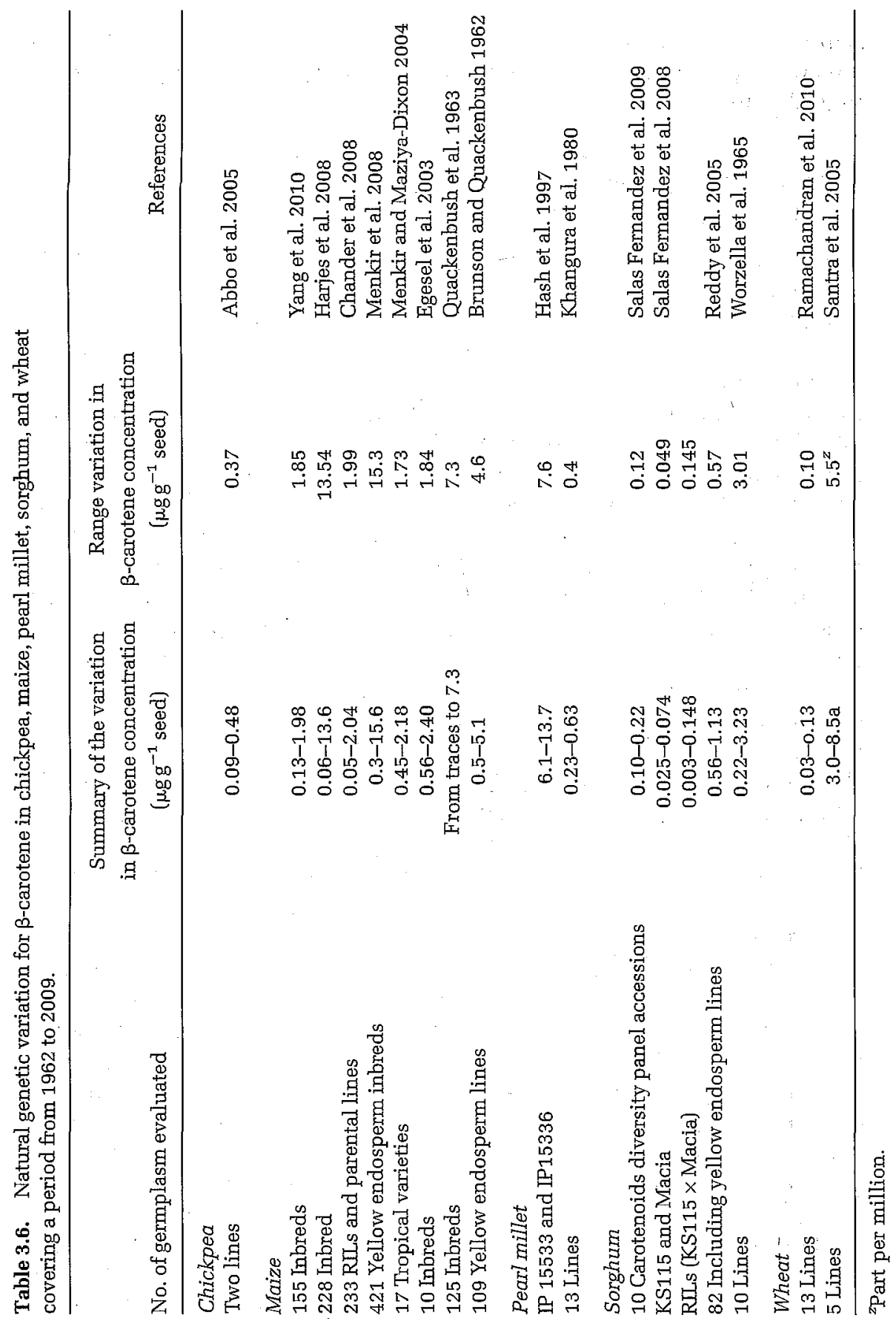


only $0.5-1.5 \mu \mathrm{gg}^{-1} \beta$-carotene. Maize has been extensively studied for molecular polymorphism, mapped QTL, chemical pathways, and genes involved in carotenoids biosynthesis, including $\beta$-carotene (Table 3.7). Carotenes are intermediates in the carotenoids biosynthetic pathway and lycopene (the immediate precursor of provitamin A carotenes) represents a branch point in this pathway, which is further modified by lycopene $\beta$-cyclase and lycopene $\varepsilon$-cyclase (LCYE) enzymes that catalyze formation of terminal $\beta$ - and $\varepsilon$-rings, respectively, to form either $\beta$-carotene, $\alpha$-carotene, or $\beta$-cryptoxanthin. This pathway continues with hydroxylation of the carotenes that depletes the provitamin A pool by converting these compounds to nonprovitamin A xanthophylls. Pathway branching and hydroxylation are therefore key determinants in controlling vitamin A levels. Polymorphism at the LCYE locus in maize explained $58 \%$ of the variation in $\alpha$ - and $\beta$-carotene and a threefold difference in provitamin A compounds (Harjes et al. 2008), while a rare genetic variant, $\beta$-carotene hydroxylase 1 (crtRB1), increases $\beta$-carotene substantially in maize grains (Yan et al. 2010). Further, metabolite sorting of a germplasm collection identified 10 genetically diverse subsets representing biochemical extremes for maize kernel carotenoids and transcript profiling of this subset led to the discovery of the Hydroxylase 3 locus that coincidently mapped to a carotene QTL (Chander et al. 2008). The natural alleles at Hydroxylase 3 locus contribute $78 \%$ of variation and approximately 11-fold differences in $\beta$-carotene relative to $\beta$-cryptoxanthin and $36 \%$ of the variation and fourfold difference in absolute levels of $\beta$-carotene (Harjes et al. 2008). The reduction in $H Y D 3$ transcripts leads to reduced conversion of $\beta$-carotene to downstream xanthophylls, causing $\beta$-carotene to accumulate (Vallabhaneni et al. 2009). Genetics tests such as the HYD3 assay (Vallabhaneni et al. 2009) together with the previously described LCYE assay (Harjes et al. 2008) may be used to select germplasm containing optimal $H Y D 3$ and $L C Y E$ alleles in breeding programs, which will lead to higher $\beta$-carotene levels in maize endosperm when both genes are highly expressed than either with optimal alleles of either gene alone. Furthermore, the experimental evidence from the association and linkage mapping reveals that $\operatorname{crtRB1}$ underlies a principal QTL associated with $\beta$-carotene concentration and conversion in maize kernels and $\operatorname{crtRB1}$ alleles associated with reduced transcript expressions correlate well with higher $\beta$-carotene (Yan et al. 2010). The most favorable $\operatorname{crtRB1}$ alleles that are rare in frequency and unique to temperate germplasm are being introgressed via inexpensive PCRmarker-assisted selection into tropical maize germplasm adapted to developing countries. A program at CIMMYT has already achieved 


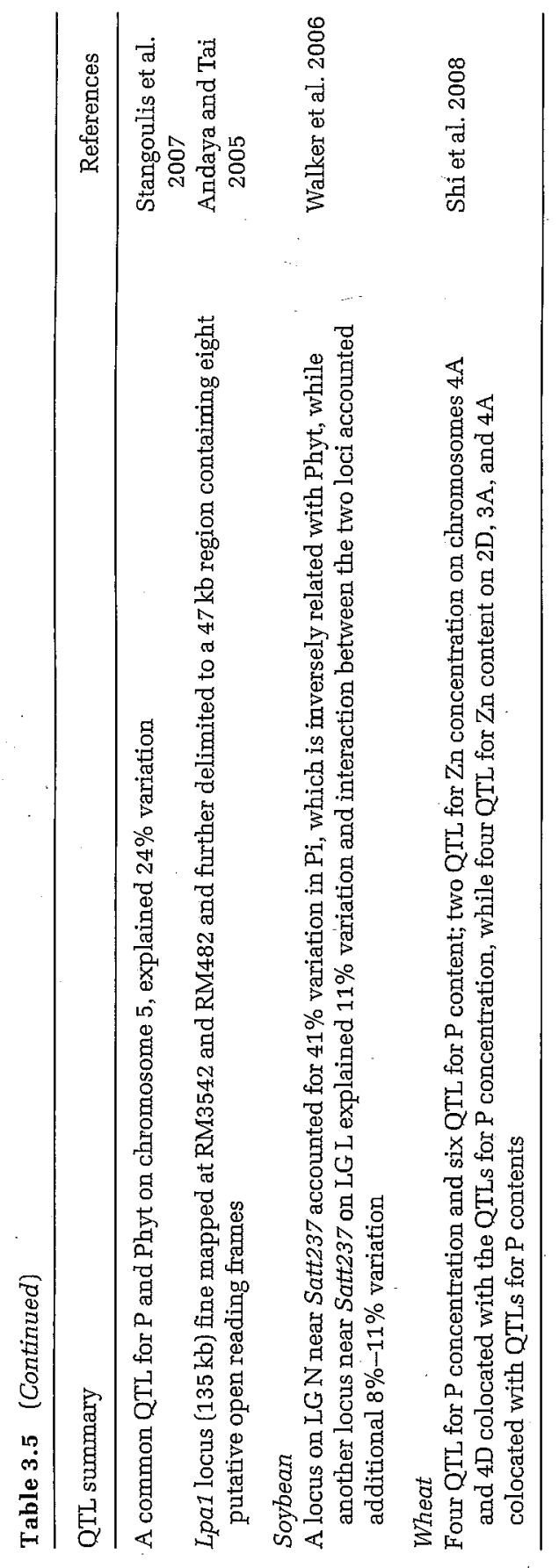


which represents a key point in the metabolism of highly phosphorylated inositols, and could be a valuable tool in plant physiology to design low-phytate crops.

\section{QTL Mapping, Cloning, and Introgression of $\beta$-Carotene into Adapted Germplasm}

The natural plant pigments, termed as carotenoids, which are fat soluble, are important source of vitamin A and antioxidants. The two groups of carotenoids are provitamin $\mathrm{A}$ and nonprovitamin $\mathrm{A}$ carotenoids. The former includes three carotenoids, namely $\beta$-carotene, $\beta$-cryptoxanthin, and $\alpha$-carotene, all precursor for vitamin $A$, while the latter consists of lutein and zeaxanthin, which act as antioxidants. Of all the carotenoids, $\beta$-carotene is the most widely distributed in plants and the one most efficiently converted to vitamin A. The consumption of carotenoid-rich foods is associated with reduced risks of developing cancer and cardiovascular diseases, enhanced immune responses, improved vision, and prevention of night blindness as well the maintenance of healthy skin and gastrointestinal or respiratory systems. Carotenoids in plants play a crucial role in photosynthesis, membrane stability, growth, and development (Menkir et al. 2008).

Unlike other agronomically important traits, only limited germplasm sets have been assessed for $\beta$-carotene, predominantly in chickpea, maize, pearl millet, sorghum, and wheat (Table 3.6), with some lines accumulating $\beta$-carotene as high as $7.6 \mu \mathrm{gg}^{-1}$ in pearl millet (Hash et al. 1997) and $15.6 \mathrm{\mu gg}^{-1}$ in maize (Harjes et al. 2008; Menkir et al. 2008). Genotypic differences for $\beta$-carotene have also been reported in rice: some germplasm had shown $\beta$-carotene in unpolished seeds while others having no $\beta$-carotene in unpolished seeds. The rice germplasm with $\beta$-carotene in unpolished seeds include Amarillo Cuba, Dudemasino, Sirendah Kuning, Bongkitan, Calibo, Khao Dawk Mali 105, and Klemas (Tan et al. 2005). Furthermore, Kandlakunta et al. (2008) in a comprehensive study involving major cereals and legumes and commonly consumed vegetables detected high $\beta$-carotene in chickpea $\left(15.7 \mu \mathrm{gg}^{-1}\right)$, green gram $\left(12.8 \mu \mathrm{gg}^{-1}\right)$, red gram $\left(12.4 \mu \mathrm{gg}^{-1}\right)$, and maize $\left(17.1 \mu \mathrm{g} \mathrm{g}^{-1}\right)$, while among vegetables, they detected high $\beta$-carotene in yellow pumpkin $\left(118 \mu \mathrm{gg}^{-1}\right)$, green chillies (102 $\left.\mu \mathrm{gg} \mathrm{g}^{\rightarrow 1}\right)$, field beans (55.4 $\left.\mu \mathrm{gg}^{-1}\right)$, French bean (39.3 $\left.\mathrm{gg} \mathrm{g}^{-1}\right)$, ridge gourd $\left(32.4 \mu \mathrm{gg}^{-1}\right)$, green beans $\left(23.9 \mu \mathrm{gg}^{-1}\right)$, and brinjal $\left(16.9 \mu \mathrm{gg}^{-1}\right)$.

Maize is an important source of provitamin $A$ ( $\beta$-carotene, $\alpha$-carotene, and $\beta$-cryptoxanthin) and the nonprovitamin $A$ including lutein and zeaxanthin. Most of the yellow maize grains consumed worldwide have 
QTL (Tiwari et al. 2010). The profiling of introgression using simple sequence repeats (SSRs), genomic in situ hybridization (GISH), and fluorescent in situ hybridization (FISH) analysis further confirmed the introgression of chromosome 2S, $2 \mathrm{U}, 7 \mathrm{~S}$, and $7 \mathrm{U}$ into these progenies (Singh et al. 2010a). More recently, genetic mapping identified five putative QTL for seed-Fe density and two QTL for seed-Zn density in pearl millet (Kumar et al. 2010b).

A number of QTL for seed phosphorus (P) and/or phytate concentrations have been reported in common bean, rice, sorghum, soybean, and wheat (Table 3.5), some with either major effects or colocated with QTL affecting seed-Fe or -Zn concentration. For example, a major QTL for P (19\% variation) on b01 colocated with QTL accounting 34\% variation each for seed Fe and $\mathrm{Zn}$ in common bean (Cichy et al. 2009). Furthermore, QTL for seed P or phytate concentration or content related to seed weight QTL on LGs b06, b07, and b10 (Blair et al. 2009c) or genes coding for candidate enzymes involved in phytic acid synthesis pathway and markers associated with each gene (Fileppi et al. 2010) have been mapped in common bean. In rice, the candidate gene for low-phytate mutant alleles and markers (LPA1_CAPS for 1 pa 1-1 and LPA1_InDel for Ipa1-2) showed complete cosegregation with mutant phenotypes (Zhao et al. 2008). Furthermore, the two QTL for seed-Zn concentration on chromosomes $4 \mathrm{~A}$ and $4 \mathrm{D}$ colocated with QTL for P concentration, while four QTL for seed-Zn content on chromosome 2D, 3A, and 4A colocated with the QTL for $\mathrm{P}$ content, reflecting positive correlation between the seed-Zn and -P concentrations (see Section VI.B), which may provide opportunities for simultaneous improvement in seed-P and -Zn density in wheat (Shi et al. 2008).

Two QTL mapped onto LGs L and N control low phytate in soybean line, CX 1834 (Walker et al. 2006; Gao et al. 2008). Further, Saghai-Maroof et al. (2009) mapped and sequenced a putative multidrug resistanceassociated protein (MRP) gene on LG $N$ that contributes to low-phytate phenotype in CX 1834. This A to T mutation provided a single nucleotide polymorphism (SNP) marker for introgressing the low-phytate QTL from CX 1834 into desired breeding lines (Saghai-Maroof et al. 2009).

More recently, genes coding for candidate enzymes involved in the phytic acid pathway have been mapped and identified markers associated with each gene (PvMIPSs, PVMIPSV, PVIMP, PVMIK, PVIPK2, PvITPKó, PVITPK $\beta$, PvIPK1), which may represent a useful resource to select genetic variants with low-phytate trait in common bean (Fileppi et al. 2010). Furthermore, González et al. (2010) discovered how phytate is produced in plants, by solving the structure of the protein $\operatorname{InsP}_{5} 2-$ Kinase $\left(\mathrm{IP}_{5} 2-\mathrm{K}\right)$, a distant member of the IPK family, 


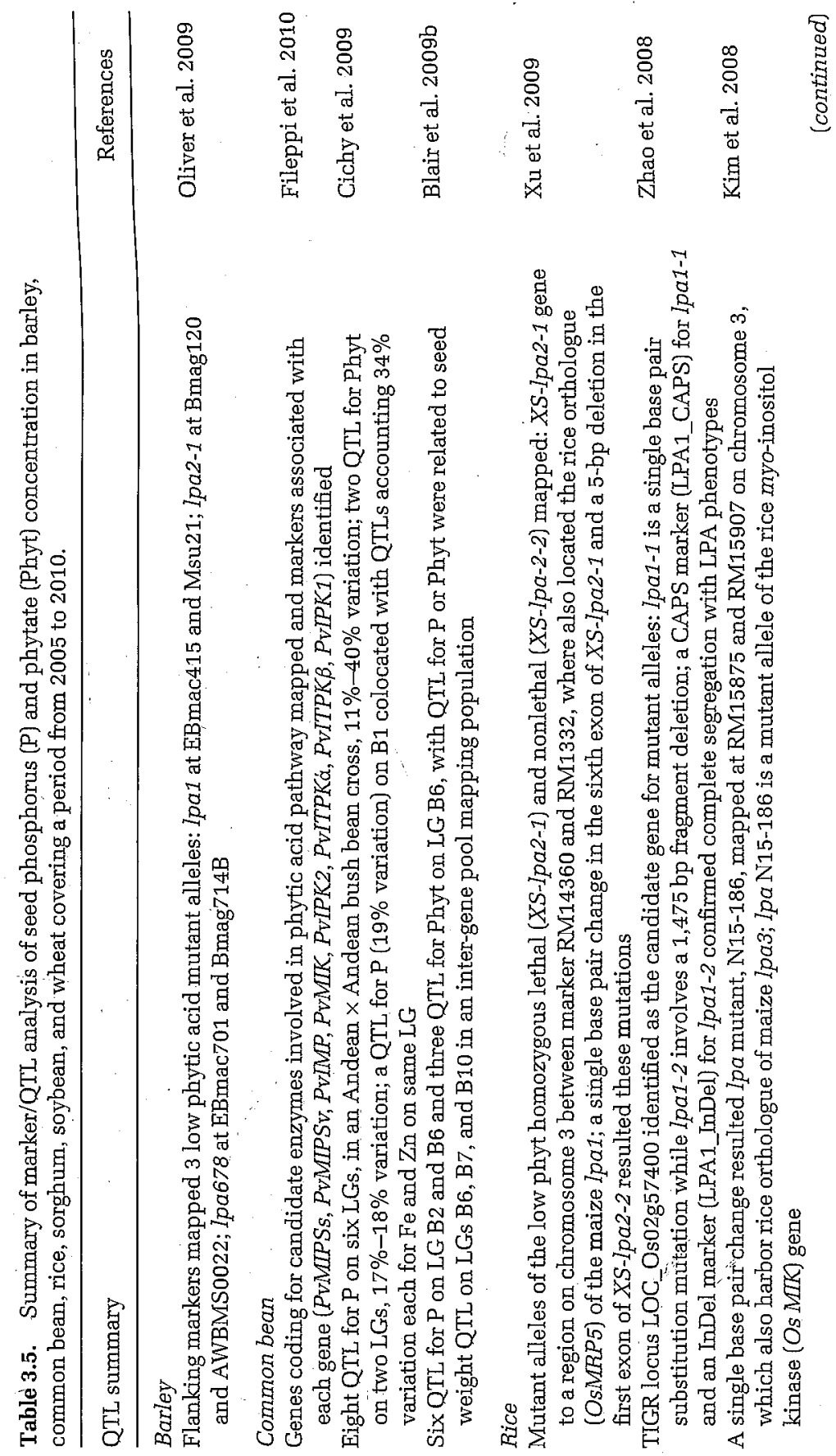




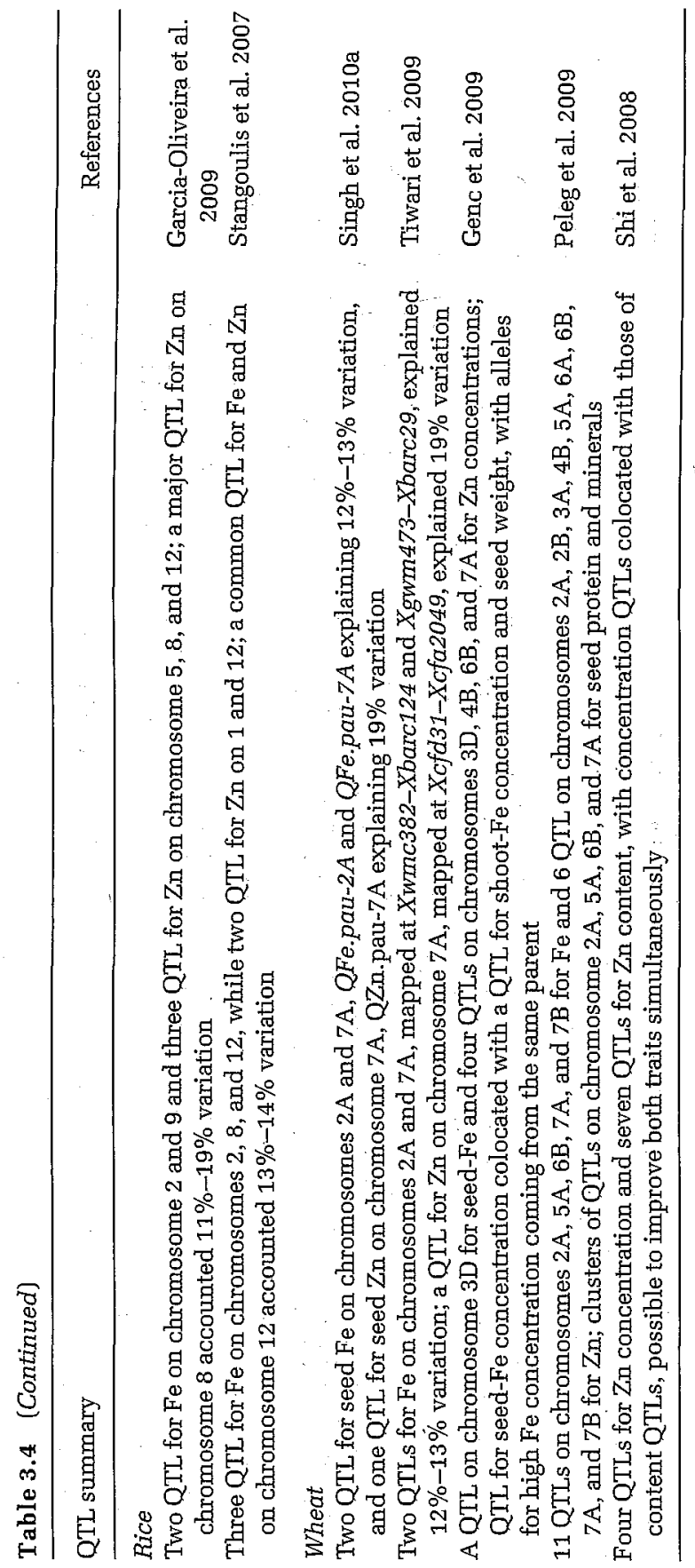


phenotypic variation (Stangoulis et al. 2007). In barley, specific. markers have been developed for a high zinc QTL on chromosome $2 \mathrm{H}$ (Sadeghzadeh et al. 2010), while other QTLs have been identified by Lonergan et al. (2009).

Iron reductases are members of the protein super-family of flavocytochromes and function in roots to convert $F$ e from a plant unavailable form (ferric, $\mathrm{Fe}^{3+}$ ) to an available form (ferrous, $\mathrm{Fe}^{2+}$ ) that can be readily absorbed (Grusak 1995). IRA is known to vary with plant growth conditions (e.g., soil $\mathrm{pH}$ and available iron concentration) (Grusak 2000). Common bean genotypes with high seed-Fe showed high IRA than those with low seed-Fe, suggesting a link between root uptake and seed loading of $\mathrm{Fe}$ in common bean (Grusak 1994, 2000, 2002). More recently, Blair et al. (2010b) reported a single major QTL for IRA under Fe-limited conditions ( $1 \mu \mathrm{M})$ on LG b02, and another major QTL under Fe-sufficient conditions $(15 \mu \mathrm{M})$ on LG b11 that was associated with several QTL for seed Fe in common bean. Thus, the QTL for IRA under Fe-limited conditions may be useful in environments where beans are grown in alkaline soils, while the QTL for IRA under Fe-sufficient conditions may be useful for selecting for enhanced seed nutritional quality (Blair et al. 2010b).

Wild emmer wheat germplasm harbors a rich allelic diversity, including for seed minerals (Xie and Nevo 2008). A major locus, Gpc-B1 (a 250-kb locus) mapped as a simple Mendelian locus (Distelfeld et al. 2006 ), associated with increased seed-protein (38\%), $-\mathrm{Fe}(18 \%)$, and $-\mathrm{Zn}$ $(12 \%)$ concentrations from wild emmer wheat germplasm (Triticum dicoccoides), encodes a NAC transcription factor (NAM-B1) that accelerates senescence and increases nutrient remobilization from leaves to developing seeds (Uauy et al. 2006; Distelfeld et al. 2007). Triticum turgidum is another useful wild emmer germplasm for improving seed mineral concentration in wheat. Peleg et al. (2009) mapped 82 QTL for 10 seed minerals (LOD score range of 3-17), with most of the positive alleles contributed by wild emmer accession, G18-16, and many QTL for the same trait mapped to homoeologous positions, reflecting synteny between the $\mathrm{A}$ and $\mathrm{B}$ genomes. TtNAM-B1 affecting seed-protein, -Fe, and $-\mathrm{Zn}$ originating from wild emmer wheat has been cloned (Distelfeld and Fahima 2007). Furthermore, Singh et al. (2010a) identified two QTL (QFe.pau-2A and QFe.pau-7A) for Fe and a QTL (QZn.pau-7A) for $\mathrm{Zn}$, which they transferred into interspecific progenies involving Aegilops kotschyi and Aegilops peregrine, both UUSS genome species. Such progenies showed 60\%-136\% enhanced seed-Fe and -Zn concentrations and 50\%-120\% increased Fe and Zn contents per seed as compared to the control cultivar that was introgressed with these 
for desirable genes in crop breeding (Dwivedi et al. 2007; Collard and Mackill 2008). Automation of high-throughput assays including next generation sequencing technologies and associated data mining tools provide breeders/molecular biologists opportunities to handle and interpret large data sets (Varshney et al. 2009; Feuillet et al. 2010). Furthermore, the genomes of agriculturally important crops such as maize, rice, sorghum, and soybean have been sequenced (IRGSP 2005; Paterson et al. 2009; Schanable et al. 2009; Schmutz et al. 2010), while several projects under way to sequence genomes of many other agriculturally important food crops (Feuillet et : al. 2010). The deoxyribonucleic acid (DNA) sequence variants across species or among strains within a species may be used as new genetic tools for developing markers and subsequently crop cultivars with specific characteristics.

The crops included in this review have abundant genetic resources to dissect population structure and diversity in germplasm collections to identify genetically diverse germplasm with beneficial traits. However, only recently have DNA marker-based technologies been used to identify QTL associated with increased seed-Fe and $-\mathrm{Zn}$ concentrations in barley, common bean, pearl millet, rice, and wheat, revealing many QTL with varying effects; some with major phenotypic variation while many others with minor effects (Table 3.4). For example, in common bean a QTL on linkage group (LG) b09 was found for Zn by Gelin et al. (2007), while QTL found by Cichy et al. (2009) on LG b01 (near the fin gene) accounted for $34 \%$ of variation for seed-Fe and -Zn concentrations and also overlapped with a major QTL (19\% variation) for increased seed phosphorus (P) concentration. Finally, other QTL on LG b06 accounted for 36\% variation for seed Fe and $39 \%$ variation for seed $\mathrm{Zn}$ that same study (Cichy et al. 2009). This latter QTL was linked with a QTL found for Mesoamerican beans by Blair et al. (2010c). Further studies by Blair et al. (2009b, 2010b) in both inter- and intragene pool populations, respectively, found specific major and minor QTL for Fe and Zn concentrations with the former type mainly on LG b11. Therefore, at least four major QTL have been identified in common bean affecting micronutrient concentration depending on the gene pool and genetic background of the material tested.

In other crops, meanwhile, a QTL for seed-Zn on chromosome 7A mapped at Xcfd31-Xcfa2049 explained 19\% variation in wheat (Tiwari et al. 2009). In rice, a seed-Zn QTL mapped at RM235-RM17 on chromosome 12 accounted for $13 \%$ variation and colocated with seed-Fe QTL that mapped at RM270-RM17 and accounted $14 \%$ 


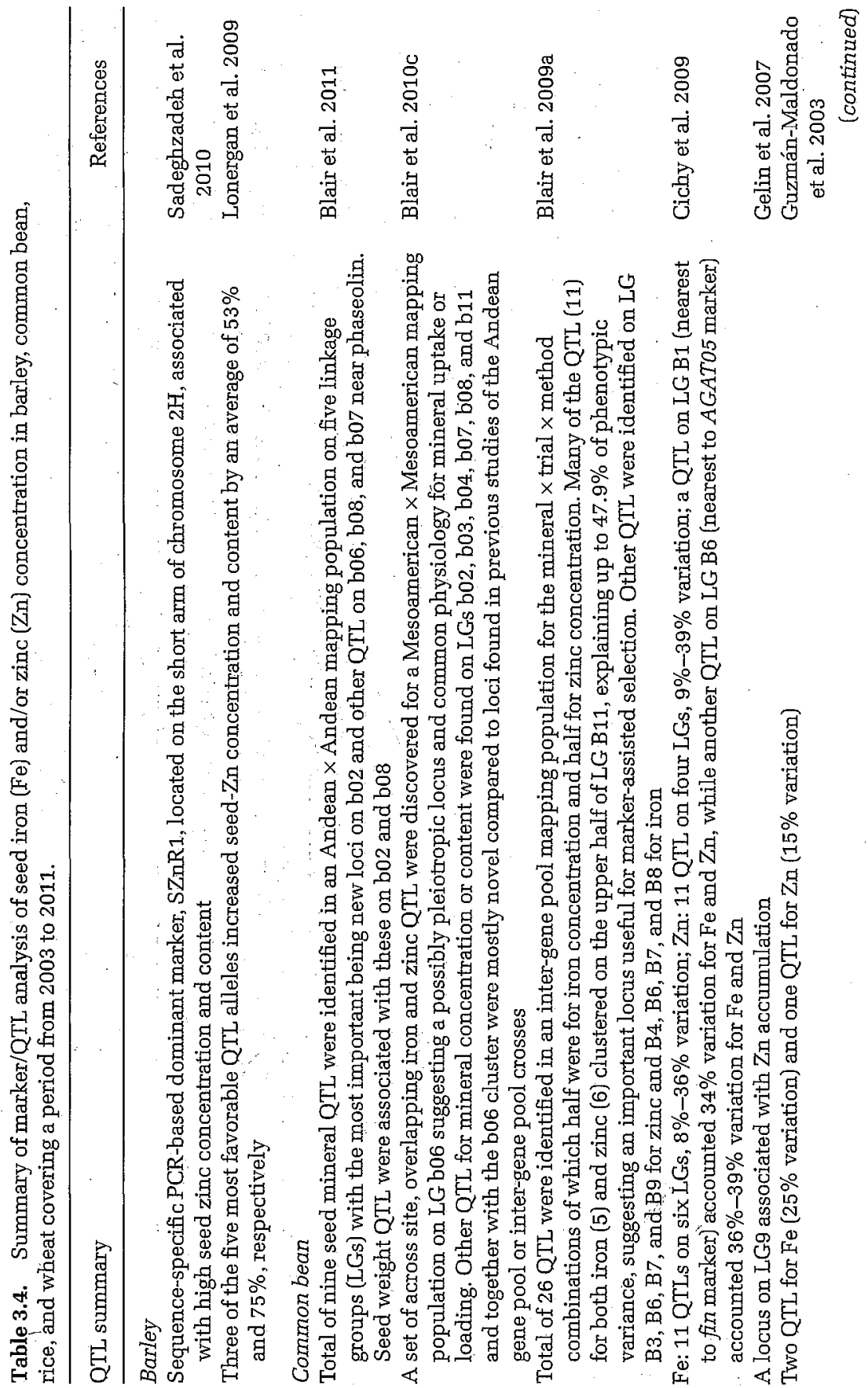




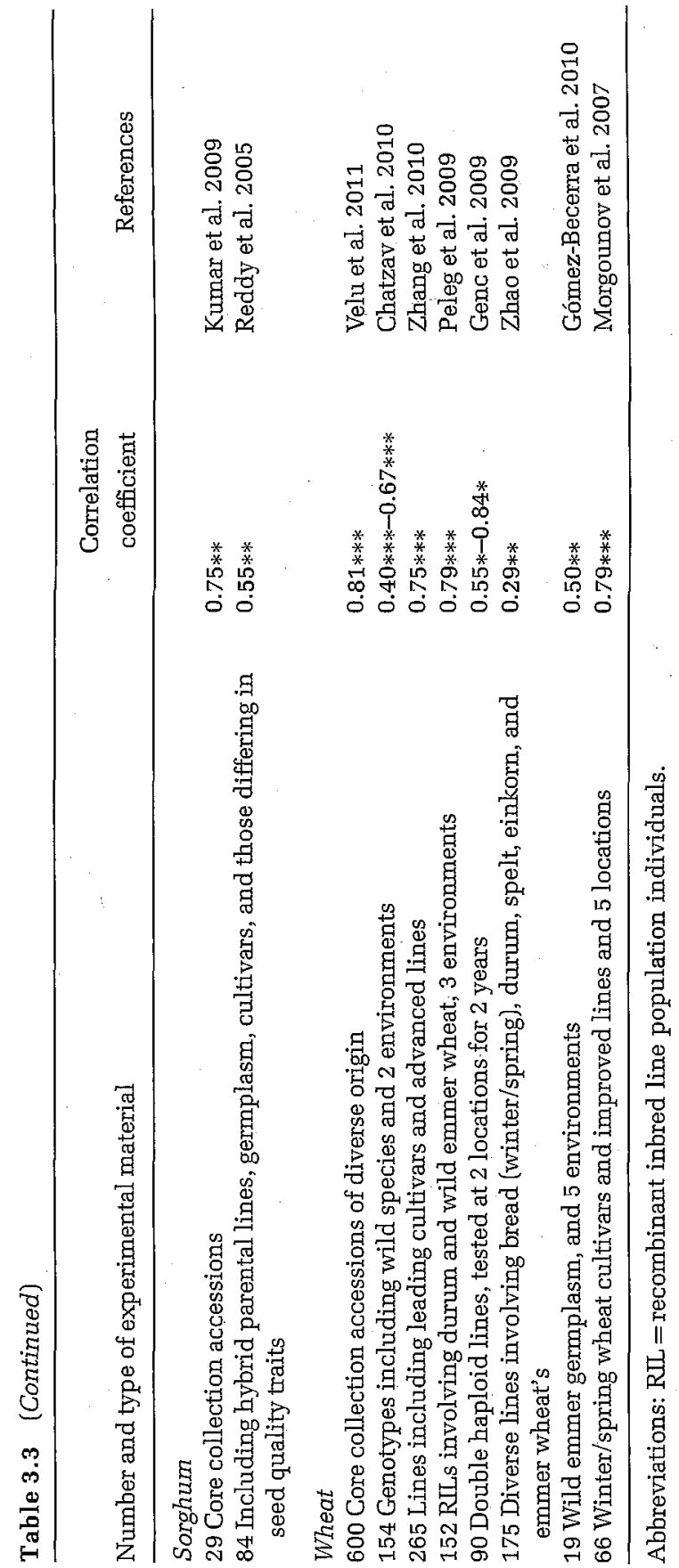


Highly significant and positive correlations (0.82-0.99) between seed $\mathrm{P}$ and phytate have been reported for common bean, pearl millet, and rice (Lolas and Markakis 1975; Stangoulis et al. 2007; Selvi and. Rajarathinam 2009), while a low but positive and significant association was found for seed phytate with $\mathrm{Fe}$ and $\mathrm{Zn}$ in common bean (Cichy et al. 2009). Further, several studies in wheat and common bean revealed a moderate but positive and significant association of seed $\mathrm{P}$ with $\mathrm{Fe}$ (0.42-0.55) and Zn (0.46-0.63) (Gelin et al. 2007; Peleg et al. 2009; Zhao et al. 2009; Zhang et al. 2010). The implications of the above are that while it should be possible to breed for high seed-Fe and -Zn concentrations with reduced phytate concentration in pearl millet, this may not be possible in wheat or common bean.

In yet another issue of correlations and micronutrient versus macronutrient concentrations, seed yield is significantly and positively associated with seed weight in cereals and legumes (Upadhyaya et al. 2002; García del Moral et al. 2003; Upadhyaya 2003; Maman et al. 2004; Morgounov et al. 2007). However, it is either not associated with seed$\mathrm{Fe}$ and $-\mathrm{Zn}$ concentrations in pearl millet or shows a low but significant negative association in wheat (Morgounov et al. 2007; Peleg et al. 2009; Zhao et al. 2009) and positive association in common bean (Gelin et al. 2007). A negative association may pose problems for breeding of seed mineral-dense cultivars with high seed yield per se. Seed weight in pearl millet is highly significant and positively associated with seed $\mathrm{Fe}$ $(r=0.80)$ and $\mathrm{Zn}(r=0.85)$ (Velu et al. 2007), while it is significant and positively correlated $(r=0.61)$ with Fe in common bean (Gelin et al. 2007).

Another question is whether there are any relationships between seed-Fe (or - Zn) concentration and bioavailability. Limited studies on seed-Fe and -Zn concentration and bioavailability in maize, rice, and wheat revealed no such associations (Glahn et al. 2002; Oikeh et al. 2003a,b,2004a), indicating that it is possible to significantly increase both concentration and bioavailability of either Fe or $\mathrm{Zn}$ in the seed by breeding and selection.

\section{Quantitative Trait Loci (QTL) Associated with Seed Iron, Zinc, and Phytate Concentrations}

Genomics science since the 1990s has made phenomenal advances toward developing a large number of molecular markers and genetic linkage maps allowing the mapping and/or cloning of QTL and identification of candidate gene(s) associated with agriculturally beneficial traits, which can lead marker-assisted selection (MAS) 
gradually from the aleurone/pericarp and outer parts of the endosperm to the interior of the endosperm, while $\mathrm{Mn}$ and Fe very much localize in the aleurone/pericarp region with a sharp change in the concentration in the exterior parts of the endosperm. $\mathrm{Mn}$ is highly concentrated in the embryo but with a different pattern than observed for Zn. The strong similarities between the distribution of Fe, Mn, and P and between Zn and $S$ may be indicative of the complexation mechanisms involved in rice seeds. Preliminary studies in pearl millet revealed greater concentration of seed minerals including $\mathrm{Fe}$ and $\mathrm{Zn}$ in the covering layers and the germ than in the endosperm portions, similar to most cereal seeds (Varriano-Marston and Hoseney 1980).

Common bean and soybean genotypes were reported to accumulate different proportion of total seed $F$ in the seed coat, embryo, and cotyledons (Laszlo 1990; Moraghan and Grafton 2002; Moraghan et al. 2002; Moraghan 2004; Ariza-Nieto et al. 2007; Cvitanich et al. 2010, 2011), indicating that specific tissues relevant for Fe storage should be identified and their $\mathrm{Fe}$ loading mechanisms be investigated to exploit such variability toward developing seed iron-dense cultivars. Using PIXE assay to investigate Fe distribution in seed tissues of Phaseolus species, Cvitanich et al. (2010, 2011) concluded that (1) the distribution of Fe in seed depends on the species and genotype, (2) high concentrations of $\mathrm{Fe}$ accumulate in cells surrounding the provascular tissue, (3) the tissue in the proximity of the provascular bundles holds up to $500 \mu \mathrm{gg}^{-1}$ Fe, depending on genotypes, and (4) the largest proportion of seed Fe in Phaseolus species is stored in compounds and cell parts different from ferritin and starch vacuoles. These results indicate that more studies are needed to assess the patterns of micronutrient distribution in seeds, and that micronutrient distribution criteria should be integrated into the selection strategies for biofortification of staple crops.

In summary, CGIAR and national agricultural research systems (NARS) institutions hold large collection of germplasm, both cultivated and wild relatives' of cereal and legume crops. The core or mini-core collections available in these crops may be used to identify seed mineral-dense germplasm. The germplasm from regions deficient in soil micronutrients should receive priority for evaluation as such germplasm are expected to develop inherent adaptation mechanisms that favor enhanced nutrient acquisition, transport, distribution, and relocation in plants/seeds. Few germplasm lines with high seed-Fe and/or -Zn concentrations have been reported in common bean, maize, pearl millet, rice, sorghum, and wheat. Wild and weedy relatives of common bean and wheat have shown abundant variability for Fe and $\mathrm{Zn}$. Mutants with moderate-to-high reduction in phytate are available in 
barley, common bean, maize, rice, soybean, and wheat. Genotypic differences in iron bioavailability have been reported in common bean, maize, rice, and wheat, which should be further explored. Several methods are available with high precision to map elements distribution, which may be used to identify barriers to Fe and Zn accumulation in the seed.

\section{EXPLOITING NATURAL GENETIC VARIATION TO BREED FOR SEED MINERAL-DENSE CULTIVARS}

\section{A. Fixing the Biologically Attainable Target to Breed for Seed Mineral-Dense Crops}

Several factors must be taken into consideration when setting the target levels for enhancing the nutritional status of food crops by breeding. These include (1) mapping the human populations with micronutrient deficiency, (2) food habits of those suffering from micronutrient malnutrition, (3) the major staple crops grown in micronutrient-deficient regions and their nutrient profiles, (4) the recommended micronutrient requirement vis-à-vis daily nutrient intake, (5) the genetic variation for micronutrients in germplasm pools and cultivars/hybrids produced in the region or of possible production there, and (6) the bioavailability, bioconversion, and bioaccessibility of the micronutrients in the crop or combination of crops consumed in the diet (Nestel et al. 2006; Ortiz-Monasterio et al. 2007; Pfeiffer and McClafferty 2007; Bouis and Welch 2010). The target set for crop biofortification for one nutrient may not be the same for a different micronutrient, and may further differ from one country or region to another. The baseline data of daily intake of minerals may vary as detected for iron concentration in India. The intake of iron in India is less than $50 \%$ of the recommended dietary allowance, and iron density is about $8.5 \mathrm{mg} 1000 \mathrm{Kcal}^{-1}$, with significant differences in absolute amounts among regions. Diets in Indian state of Andhra Pradesh with rice as staple have lowest iron ( $7 \mathrm{mg} 1000$ $\mathrm{Kcal}^{-1}$ ), while diets in Gujarat and Madhya Pradesh with pearl millet as the staple have the highest iron intake (16 mg $1000 \mathrm{Kcal}^{-1}$ ) (Nair and Iyengar 2009). Likewise, several target regions for crop biofortification ( $\mathrm{Fe}, \mathrm{Zn}$, and provitamin A) interventions have been ideńtified in Latin America and the Caribbean (Zapata-Caldas et al. 2009). For example, interventions in northern Colombia appear promising for all crops, while sites for bean biofortification are widely scattered throughout the country. The most promising sites in Nicaragua are found in the 
center-north region, while candidate sites for biofortification in Bolivia are found in the central part of the country and in the Andes Mountains. Poverty levels indicated that northeast Brazil is the most important region for biofortification in that part of South America.

Variations in the conversion factor of provitamin A ( $\beta$-carotene) to vitamin A (retinol) in food crops should also need to be considered when defining breeding targets for $\beta$-carotene (Tang 2010). Significant genetic variation for seed-Fe and - Zn concentrations has been reported for cereal and legume crops, with some genotypes having more bioavailable seed micronutrients than others (see Section V.A). Most modern cultivars/hybrids have lower micronutrients per se than those reported in germplasm pools of a given species (Graham et al. 1999; Frossard et al. 2000). The adverse effects of processing, storage, and cooking on nutrient concentrations losses are known (see Section III.B.2). In addition, there are certain elements present in the seed that either act as enhancers (i.e., ascorbic acid) or inhibitors (i.e., phytase) of micronutrient uptake and absorption (see Section III.B.1).

All these variables need to be factored in when setting the breeding targets for improved nutritional quality of food crops. HarvestPlus has set the tentative breeding targets for improving the micronutrient density of several food crops. For example, the tentative targets to increase seed-Fe concentration of rice, wheat, pearl millet, common beans, maize, cassava, and sweet potato are $15,59,88,107,60,45$, and $85 \mu g g^{-1}$ on a dry weight basis, respectively, while those for $\mathrm{Zn}$ are fixed at 28, 38, 66, 56, 38, 34, and $70 \mu \mathrm{gg}^{-1}$ (Bouis and Welch 2010).

For provitamin $A$, the targets set are $17,17,23,34,17,48$, and $91 \mu \mathrm{gg}^{-1}$ for rice, wheat, pearl millet, common bean, maize, cassava, and sweet potato, respectively (Bouis and Welch 2010). Such an approach can be applied to define the target levels for other micronutrients as well. However, targets should be dynamic depending on the severity of the micronutrient deficiency and the progress realized through breeding for developing mineral-dense cultivars/hybrids.

\section{B. Genotype $\times$ Environment Interaction and Relationships Between Seed Minerals and Agronomic Traits}

Knowledge of the effects of $G$, environments (E), and genotype $\times$ environment interaction (GEI) is important for developing nutritionally enhanced crop cultivars. Like yield and yield attributing traits, seedFe and -Zn concentrations in common bean, maize, rice, and wheat are influenced by location (or E), G, and GEI (Table 3.2), with location effects generally much larger than those of either $\mathrm{G}$ or GEI effects. The 


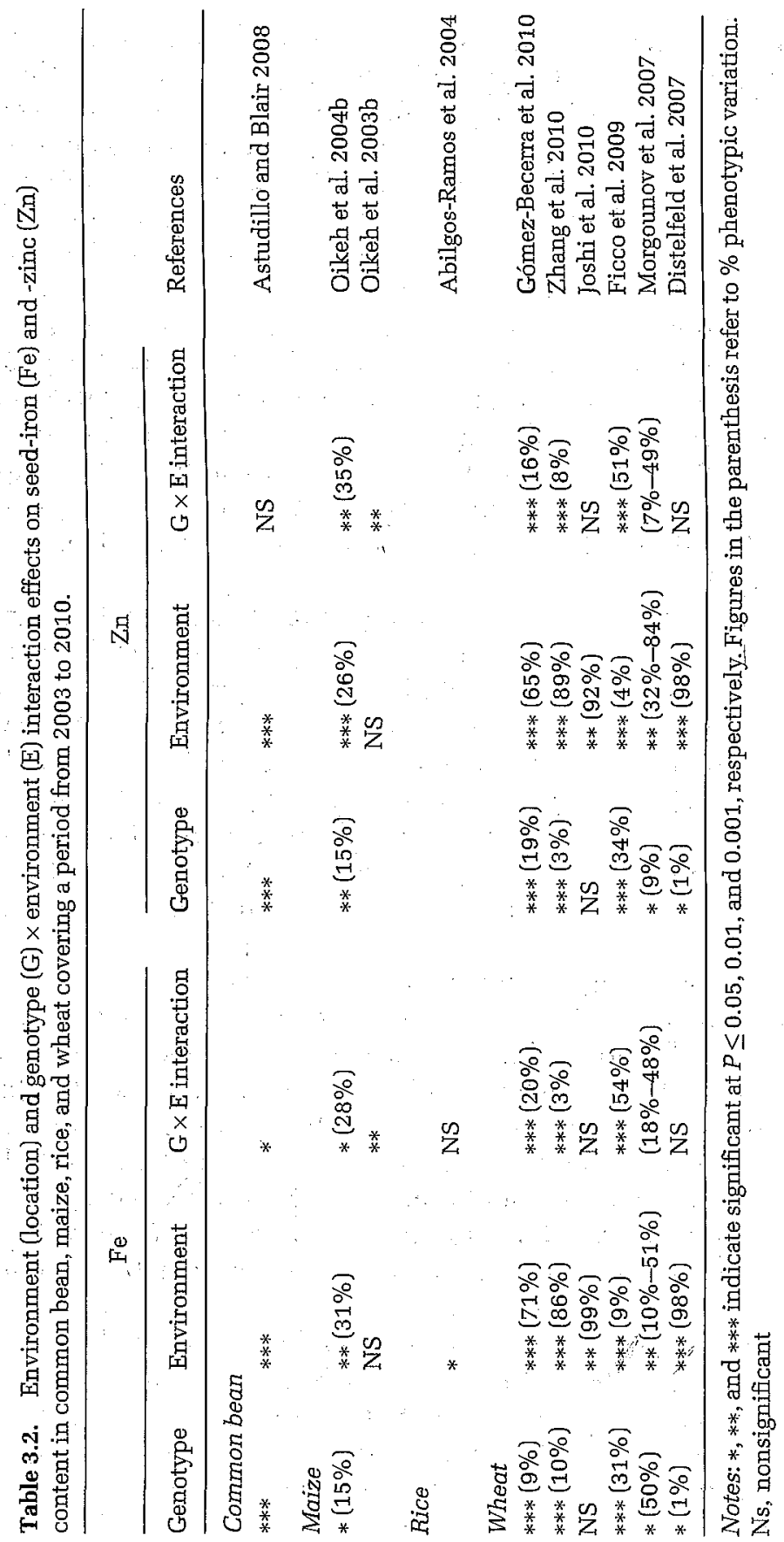


growing environments had no effect on bioavailable Fe in maize (Oikeh et al. 2004a); however, Pixley et al. (2011a) detected larger E than GEI for $\mathrm{Fe}$ bioavailability in maize. It is therefore suggested that responses of cultivars to different production environments need to be well understood to improve the probability of predicting and identifying cultivars that are not only high in seed-Fe and/or - $\mathrm{Zn}$ concentrations but also these micronutrients are more bioavailable to absorption (Briat and Lobreaux 1997; Pixley et al. 2011a).

The environmental variables such as $\mathrm{pH}$, temperature, solar radiation, precipitation, organic matter, and soil texture have the potential to influence nutrient concentration (Tisdale and Nelson 1975; Römheld and Marschner 1986; Cabuslay et al. 2003; Abilgos-Ramos et al. 2004; Joshi et al. 2010) and must be taken into consideration while explaining the variation for seed micronutrients in germplasm or when assessing the nutritional quality of staple food crops grown in diverse agroecological conditions.

Character association between seed mineral concentrations may indicate the existence of one or more common genetic-physiological mechanisms involved in mineral uptake by the root system, translocation, and redistribution within the plant tissues, remobilization to the seed, and accumulation in the developing seed (Chatzav et al. 2010). Both mineral concentration (amount per unit weight, i.e., $\mathrm{mg} \mathrm{kg}^{-1}$ ) and mineral content (amount per seed, i.e., $\mu$ g seed $^{-1}$ ) are positively correlated (Cakmak et al. 2004; Hacisalihoglu et al. 2005; Stangoulis et al. 2007) and either can be used to estimate the quantity of the minerals in the seeds (see Section V.A).

An understanding of the nature of association between different minerals and also with seed yield and seed weight (100 or 1,000seed weight) should facilitate the selection of mineral-dense progenies in breeding. The published evidence suggests that seed-Fe and $-\mathrm{Zn}$ concentrations, in most cases, are highly significant and positively correlated in common bean, pearl millet, rice, and wheat (Table 3.3), which suggests that genes for $\mathrm{Fe}$ and $\mathrm{Zn}$ accumulation cosegregate or are pleiotropic. Such relationships could be exploited toward selecting progenies with high seed minerals in the segregating populations. Further studies revealed that $\mathrm{Fe}$ and $\mathrm{Zn}$ in the flag leaves of Aegilops species are highly significant and positively correlated with seed Fe and Zn (Rawat et al. 2009a,b); however, such relationships were not found in common bean (Tryphone and Nchimbi-Msolla 2010). More studies are needed to elucidate these relationships prior to using flag leaf for early selection of plants with potentially high seed $\mathrm{Fe}$ and $\mathrm{Zn}$ in breeding programs. 


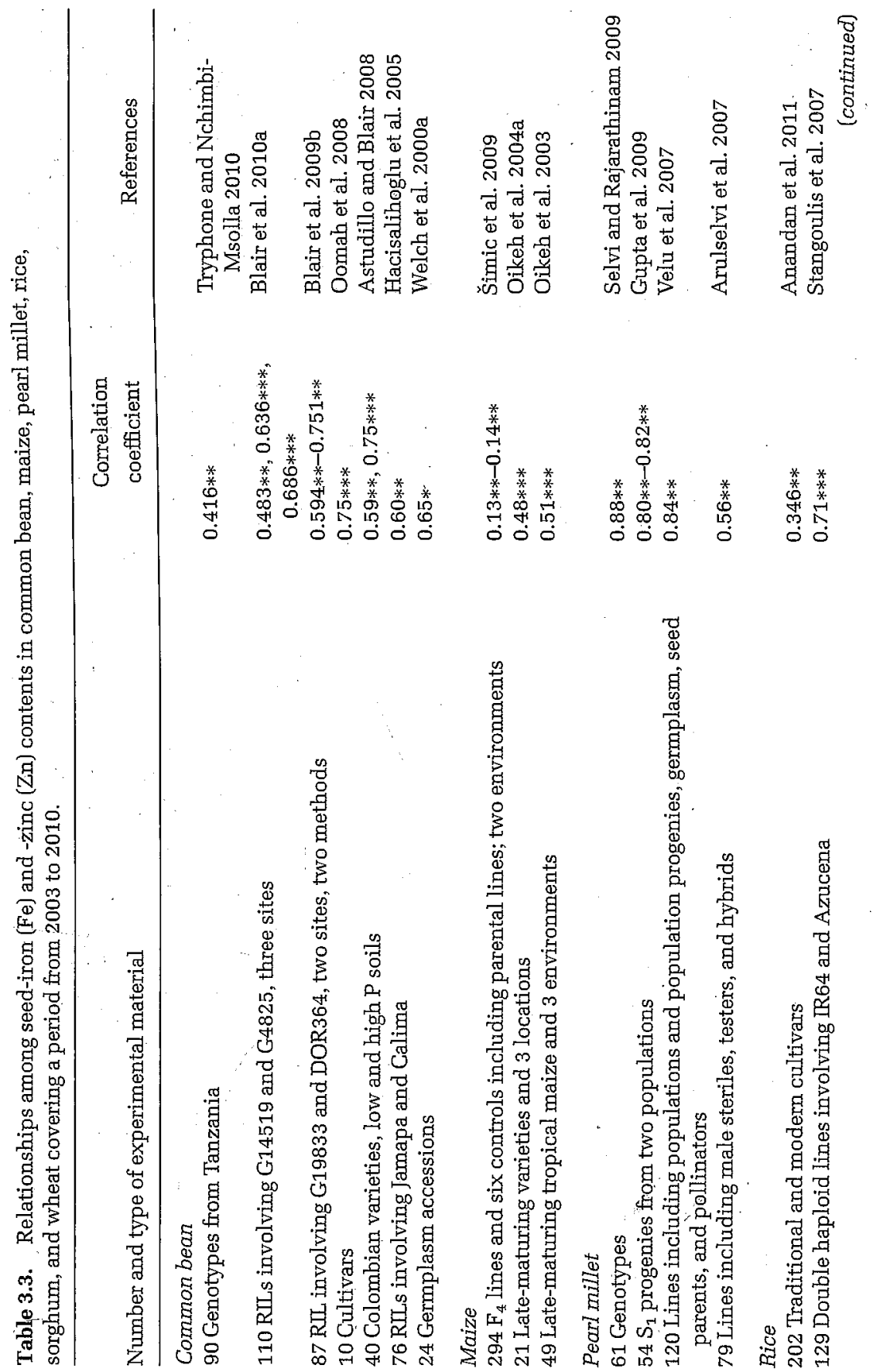




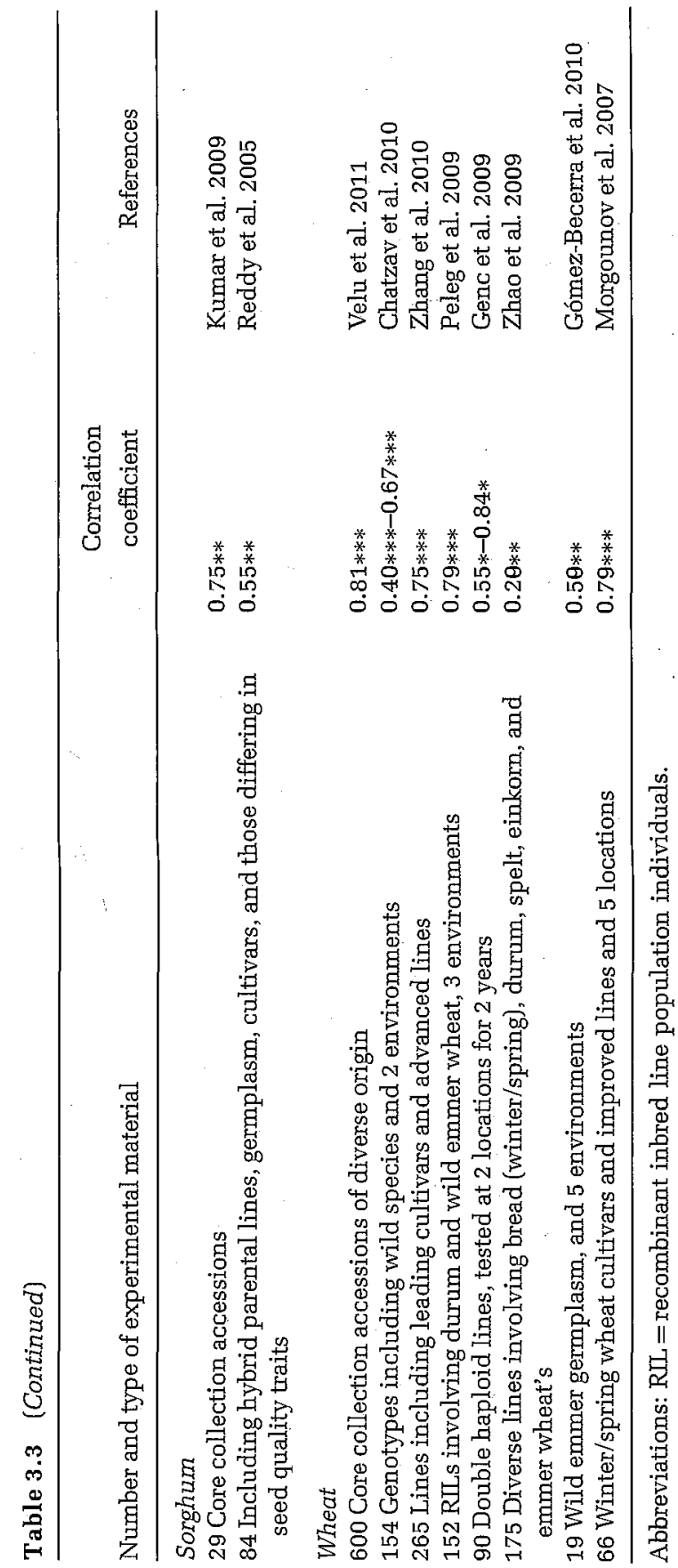


Highly significant and positive correlations (0.82-0.99) between seed $\mathrm{P}$ and phytate have been reported for common bean, pearl millet, and rice (Lolas and Markakis 1975; Stangoulis et al. 2007; Selvi and Rajarathinam 2009), while a low but positive and significant association was found for seed phytate with Fe and $\mathrm{Zn}$ in common bean (Cichy et al. 2009). Further, several studies in wheat and common bean revealed a moderate but positive and significant association of seed $\mathrm{P}$ with $\mathrm{Fe}$ (0.42-0.55) and Zn (0.46-0.63) (Gelin et al. 2007; Peleg et al. 2009; Zhao et al. 2009; Zhang et al. 2010). The implications of the above are that while it should be possible to breed for high seed-Fe and -Zn concentrations with reduced phytate concentration in pearl millet, this may not be possible in wheat or common bean.

In yet another issue of correlations and micronutrient versus macronutrient concentrations, seed yield is significantly and positively associated with seed weight in cereals and legumes (Upadhyaya et al. 2002; García del Moral et al. 2003; Upadhyaya 2003; Maman et al. 2004; Morgounov et al. 2007). However, it is either not associated with seedFe and -Zn concentrations in pearl millet or shows a low but significant negative association in wheat (Morgounov et al. 2007; Peleg et al. 2009; Zhao et al. 2009) and positive association in common bean (Gelin et al. 2007). A negative association may pose problems for breeding of seed mineral-dense cultivars with high seed yield per se. Seed weight in pearl millet is highly significant and positively associated with seed $\mathrm{Fe}$ $(r=0.80)$ and $\mathrm{Zn}(r=0.85)$ (Velu et al. 2007), while it is significant and positively correlated $(r=0.61)$ with $\mathrm{Fe}$ in common bean (Gelin et al. 2007).

Another question is whether there are any relationships between seed-Fe (or - Zn) concentration and bioavailability. Limited studies on seed-Fe and $-\mathrm{Zn}$ concentration and bioavailability in maize, rice, and wheat revealed no such associations (Glahn et al. 2002; Oikeh et al. 2003a,b,2004a), indicating that it is possible to significantly increase both concentration and bioavailability of either $\mathrm{Fe}$ or $\mathrm{Zn}$ in the seed by breeding and selection.

\section{Quantitative Trait Loci (QTL) Associated with Seed Iron, Zinc, and Phytate Concentrations}

Genomics science since the 1990s has made phenomenal advances toward developing a large number of molecular markers and genetic linkage maps allowing the mapping and/or cloning of QTL and identification of candidate gene(s) associated with agriculturally beneficial traits, which can lead marker-assisted selection (MAS) 
for desirable genes in crop breeding (Dwivedi et al. 2007; Collard and Mackill 2008). Automation of high-throughput assays including next generation sequencing technologies and associated data mining tools provide breeders/molecular biologists opportunities to handle and interpret large data sets (Varshney et al. 2009; Feuillet et al. 2010). Furthermore, the genomes of agriculturally important crops such as maize, rice, sorghum, and soybean have been sequenced (IRGSP 2005; Paterson et al. 2009; Schanable et al. 2009; Schmutz et al. 2010), while several projects under way to sequence genomes of many other agriculturally important food crops (Feuillet et al. 2010). The deoxyribonucleic acid (DNA) sequence variants across species or among strains within a species may be used as new genetic tools for developing markers and subsequently crop cultivars with specific characteristics.

The crops included in this review have abundant genetic resources to dissect population structure and diversity in germplasm collections to identify genetically diverse germplasm with beneficial traits. However, only recently have DNA marker-based technologies been used to identify QTL associated with increased seed-Fe and - Zn concentrations in barley, common bean, pearl millet, rice, and wheat, revealing many QTL with varying effects; some with major phenotypic variation while many others with minor effects (Table 3.4). For example, in common bean a QTL on linkage group (LG) b09 was found for Zn by Gelin et al. (2007), while QTL found by Cichy et al. (2009) on LG b01 (near the fin gene) accounted for 34\% of variation for seed-Fe and -Zn concentrations and also overlapped with a major QTL (19\% variation) for increased seed phosphorus (P) concentration. Finally, other QTL on LG b06 accounted for 36\% variation for seed $\mathrm{Fe}$ and $39 \%$ variation for seed $\mathrm{Zn}$ that same study (Cichy et al. 2009). This latter QTL was linked with a QTL found for Mesoamerican beans by Blair et al. (2010c). Further studies by Blair et al. (2009b, 2010b) in both inter- and intragene pool populations, respectively, found specific major and minor QTL for Fe and Zn concentrations with the former type mainly on LG b11. Therefore, at least four major QTL have been identified in common bean affecting micronutrient concentration depending on the gene pool and genetic background of the material tested.

In other crops, meanwhile, a QTL for seed-Zn on chromosome $7 \mathrm{~A}$ mapped at Xcfd31-Xcfa2049 explained 19\% variation in wheat (Tiwari et al. 2009). In rice, a seed-Zn QTL mapped at RM235-RM17 on chromosome 12 accounted for $13 \%$ variation and colocated with seed-Fe QTL that mapped at RM270-RM17 and accounted $14 \%$ 


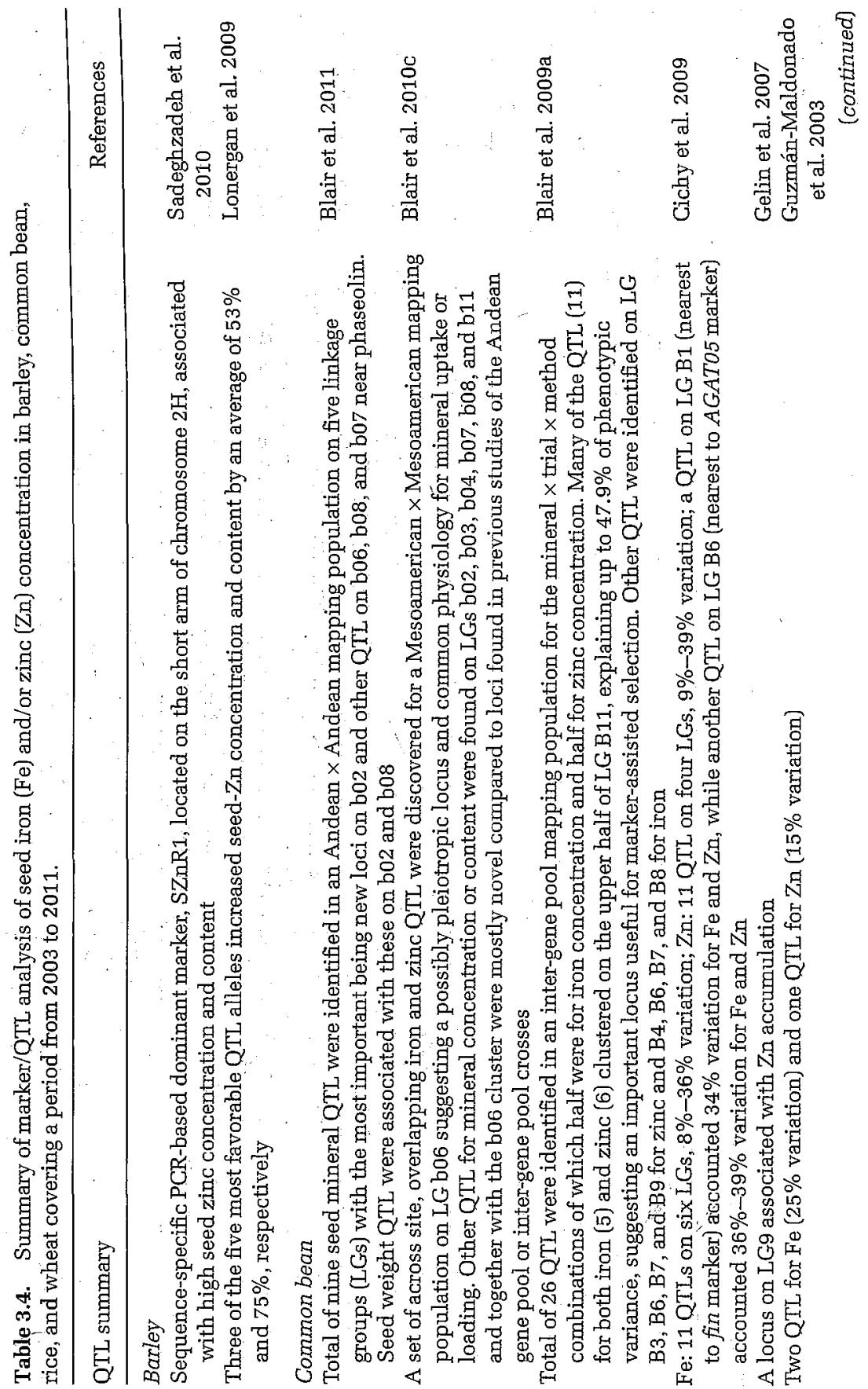




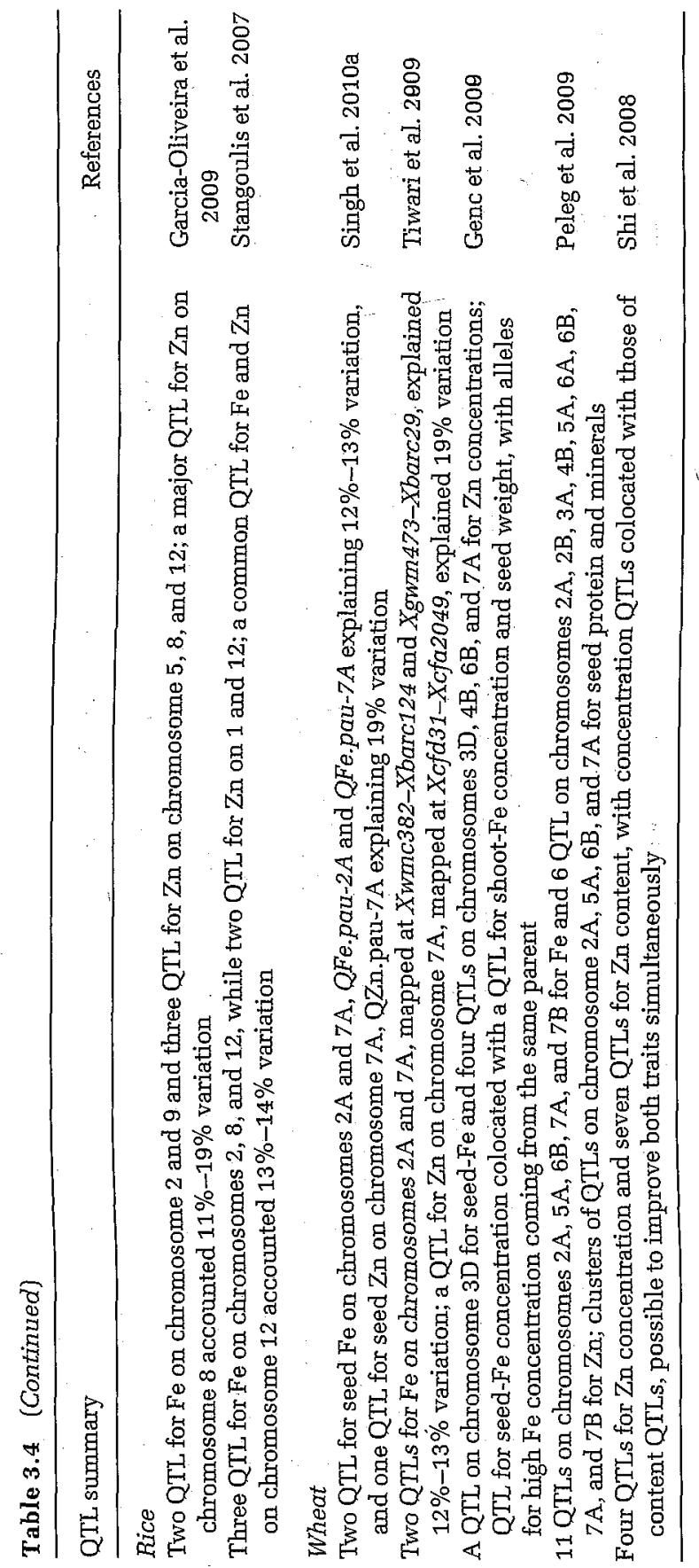


phenotypic variation (Stangoulis et al. 2007). In barley, specific markers have been developed for a high zinc QTL on chromosome 2H (Sadeghzadeh et al. 2010), while other QTLs have been identified by Lonergan et al. (2009).

Iron reductases are members of the protein super-family of flavocytochromes and function in roots to convert $F$ e from a plant unavailable form (ferric, $\mathrm{Fe}^{3+}$ ) to an available form (ferrous, $\mathrm{Fe}^{2+}$ ) that can be readily absorbed (Grusak 1995). IRA is known to vary with plant growth conditions (e.g., soil pH and available iron concentration) (Grusak 2000). Common bean genotypes with high seed-Fe showed high IRA than those with low seed-Fe, suggesting a link between root uptake and seed loading of $\mathrm{Fe}$ in common bean (Grusak 1994, 2000, 2002). More recently, Blair et al. (2010b) reported a single major QTL for IRA under Fe-limited conditions ( $1 \mu \mathrm{M})$ on LG b02, and another major QTL under Fe-sufficient conditions $(15 \mu \mathrm{M})$ on LG b11 that was associated with several QTL for seed Fe in common bean. Thus, the QTL for IRA under Fe-limited conditions may be useful in environments where beans are grown in alkaline soils, while the QTL for IRA under Fe-sufficient conditions may be useful for selecting for enhanced seed nutritional quality (Blair et al. 2010b).

Wild emmer wheat germplasm harbors a rich allelic diversity, including for seed minerals (Xie and Nevo 2008). A major locus, Gpc-B1 (a 250-kb locus) mapped as a simple Mendelian locus (Distelfeld et al. 2006), associated with increased seed-protein (38\%), -Fe (18\%), and -Zn $(12 \%)$ concentrations from wild emmer wheat germplasm (Triticum dicoccoides), encodes a NAC transcription factor (NAM-B1) that accelerates senescence and increases nutrient remobilization from leaves to developing seeds (Uauy et al. 2006; Distelfeld et al. 2007). Triticum turgidum is another useful wild emmer germplasm for improving seed mineral concentration in wheat. Peleg et al. (2009) mapped 82 QTL for 10 seed minerals (LOD score range of 3-17), with most of the positive alleles contributed by wild emmer accession, G18-16, and many QTL for the same trait mapped to homoeologous positions, reflecting synteny between the $\mathrm{A}$ and $\mathrm{B}$ genomes. TtNAM-B1 affecting seed-protein, -Fe, and -Zn originating from wild emmer wheat has been cloned (Distelfeld and Fahima 2007). Furthermore, Singh et al. (2010a) identified two QTL (QFe.pau-2A and QFe.pau-7A) for Fe and a QTL, (QZn.pau-7A) for $\mathrm{Zn}$, which they transferred into interspecific progenies involving Aegilops kotschyi and Aegilops peregrine, both UUSS genome species. Such progenies showed $60 \%-136 \%$ enhanced seed-Fe and $-\mathrm{Zn}$ concentrations and 50\%-120\% increased Fe and $\mathrm{Zn}$ contents per seed as compared to the control cultivar that was introgressed with these 
QTL (Tiwari et al. 2010). The profiling of introgression using simple sequence repeats (SSRs), genomic in situ hybridization (GISH), and fluorescent in situ hybridization (FISH) analysis further confirmed the introgression of chromosome $2 \mathrm{~S}, 2 \mathrm{U}, 7 \mathrm{~S}$, and $7 \mathrm{U}$ into these progenies (Singh et al. 2010a). More recently, genetic mapping identified five putative QTL for seed-Fe density and two QTL for seed-Zn density in pearl millet (Kumar et al. 2010b).

A number of QTL for seed phosphorus (P) and/or phytate concentrations have been reported in common bean, rice, sorghum, soybean, and wheat (Table 3.5), some with either major effects or colocated with QTL affecting seed-Fe or -Zn concentration. For example, a major QTL for P (19\% variation) on b01 colocated with QTL accounting 34\% variation each for seed $\mathrm{Fe}$ and $\mathrm{Zn}$ in common bean (Cichy et al. 2009). Furthermore, QTL for seed P or phytate concentration or content related to seed weight QTL on LGs b06, b07, and b10 (Blair et al. 2009c) or genes coding for candidate enzymes involved in phytic acid synthesis pathway and markers associated with each gene (Fileppi et al. 2010) have been mapped in common bean. In rice, the candidate gene for low-phytate mutant alleles and markers (LPA1_CAPS for Ipa1-1 and LPA1_InDel for Ipa1-2) showed complete cosegregation with mutant phenotypes. (Zhao et al. 2008). Furthermore, the two QTL for seed-Zn concentration on chromosomes 4A and 4D colocated with QTL for P concentration, while four QTL for seed-Zn content on chromosome 2D, 3A, and 4A colocated with the QTL for P content, reflecting positive correlation between the seed-Zn and -P concentrations (see Section VI.B), which may provide opportunities for simultaneous improvement in seed-P and -Zn density in wheat (Shi et al. 2008).

Two QTL mapped onto LGs L and N control low phytate in soybean line, CX 1834 (Walker et al. 2006; Gao et al. 2008). Further, Saghai-Maroof et al. (2009) mapped and sequenced a putative multidrug resistanceassociated protein (MRP) gene on LG N that contributes to low-phytate phenotype in CX 1834. This A to T mutation provided a single nucleotide polymorphism (SNP) marker for introgressing the low-phytate QTL from CX 1834 into desired breeding lines (Saghai-Maroof et al. 2009).

More recently, genes coding for candidate enzymes involved in the phytic acid pathway have been mapped and identified markers associated with each gene (PVMIPSs, PVMIPSV, PVIMP, PVMIK, PVIPK2, $P_{V I T P K} \alpha, P_{V I T P K}$, PVIPK1), which may represent a useful resource to select genetic variants with low-phytate trait in common bean (Fileppi et al. 2010). Furthermore, González et al. (2010) discovered how phytate is produced in plants, by solving the structure of the protein $\operatorname{InsP}_{5} 2$-Kinase $\left(\mathrm{IP}_{5} 2-\mathrm{K}\right)$, a distant member of the IPK family, 


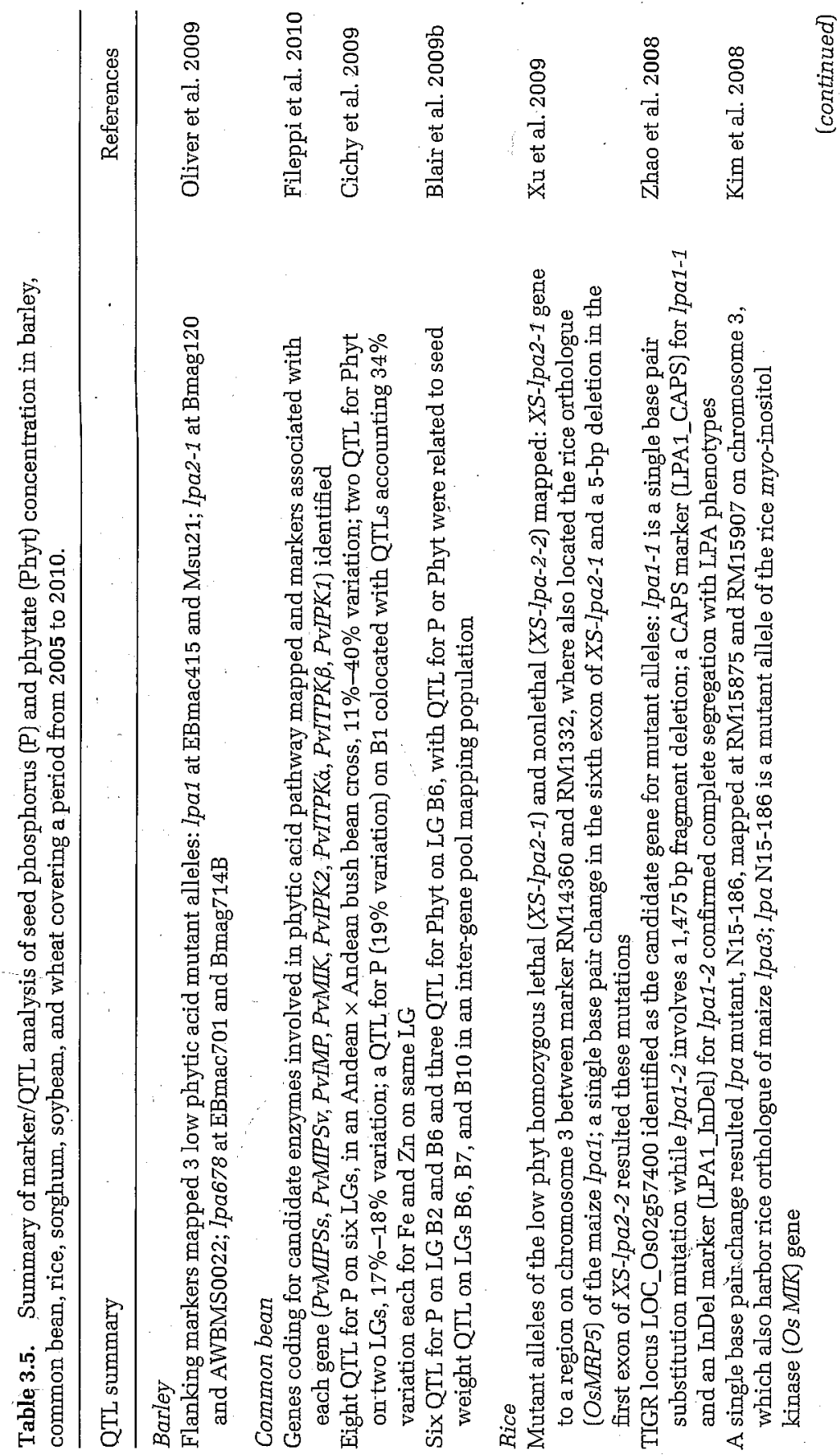




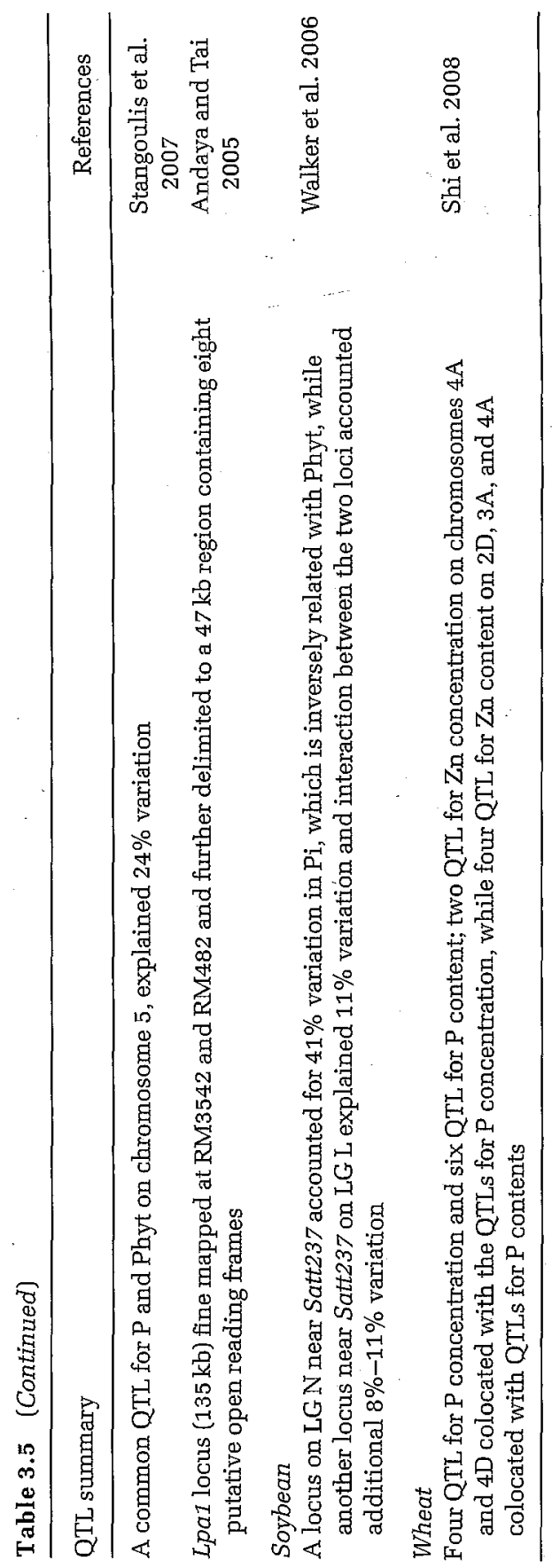


which represents a key point in the metabolism of highly phosphorylated inositols, and could be a valuable tool in plant physiology to design low-phytate crops.

\section{QTL Mapping, Cloning, and Introgression of $\beta$-Carotene into Adapted Germplasm}

The natural plant pigments, termed as carotenoids, which are fat soluble, are important source of vitamin A and antioxidants. The two groups of carotenoids are provitamin $\mathrm{A}$ and nonprovitamin $\mathrm{A}$ carotenoids. The former includes three carotenoids, namely $\beta$-carotene, $\beta$-cryptoxanthin, and $\alpha$-carotene, all precursor for vitamin A, while the latter consists of lutein and zeaxanthin, which act as antioxidants. Of all the carotenoids, $\beta$-carotene is the most widely distributed in plants and the one most efficiently converted to vitamin A. The consumption of carotenoid-rich foods is associated with reduced risks of developing cancer and cardiovascular diseases, enhanced immune responses, improved vision, and prevention of night blindness as well the maintenance of healthy skin and gastrointestinal or respiratory systems. Carotenoids in plants play a crucial role in photosynthesis, membrane stability, growth, and development (Menkir et al. 2008).

Unlike other agronomically important traits, only limited germplasm sets have been assessed for $\beta$-carotene, predominantly in chickpea, maize, pearl millet, sorghum, and wheat (Table 3.6), with some lines accumulating $\beta$-carotene as high as $7.6 \mu \mathrm{gg}^{-1}$ in pearl millet (Hash et al, 1997) and $15.6 \mu \mathrm{gg}^{-1}$ in maize (Harjes et al. 2008; Menkir et al. 2008). Genotypic differences for $\beta$-carotene have also been reported in rice: some germplasm had shown $\beta$-carotene in unpolished seeds while others having no $\beta$-carotene in unpolished seeds. The rice germplasm with $\beta$-carotene in unpolished seeds include Amarillo Cuba, Dudemasino, Sirendah Kuning, Bongkitan, Calibo, Khao Dawk Mali 105, and Klemas (Tan et al. 2005). Furthermore, Kandlakunta et al. (2008) in a comprehensive study involving major cereals and legumes and commonly consumed vegetables detected high $\beta$-carotene in chickpea $\left(15.7 \mu \mathrm{g} \mathrm{g}^{-1}\right)$, green gram $\left(12.8 \mu \mathrm{g} \mathrm{g}^{-1}\right)$, red gram $\left(12.4 \mu \mathrm{gg}^{-1}\right)$, and maize $\left(17.1 \mu \mathrm{gg}^{-1}\right)$, while among vegetables, they detected high $\beta$-carotene in yellow pumpkin $\left(118 \mu \mathrm{gg}^{-1}\right)$, green chillies $\left(102 \mu \mathrm{gg}^{-1}\right)$, field beans (55.4 $\mathrm{gg} \mathrm{g}^{-1}$ ), French bean $\left(39.3 \mu \mathrm{g} \mathrm{g}^{-1}\right)$, ridge gourd $\left(32.4 \mu \mathrm{gg}^{-1}\right)$, green beans $\left(23.9 \mu \mathrm{gg}^{-1}\right)$, and brinjal $\left(16.9 \mu \mathrm{gg}^{-1}\right)$.

Maize is an important source of provitamin A ( $\beta$-carotene, $\alpha$-carotene, and $\beta$-cryptoxanthin) and the nonprovitamin $A$ including lutein and zeaxanthin. Most of the yellow maize grains consumed worldwide have 


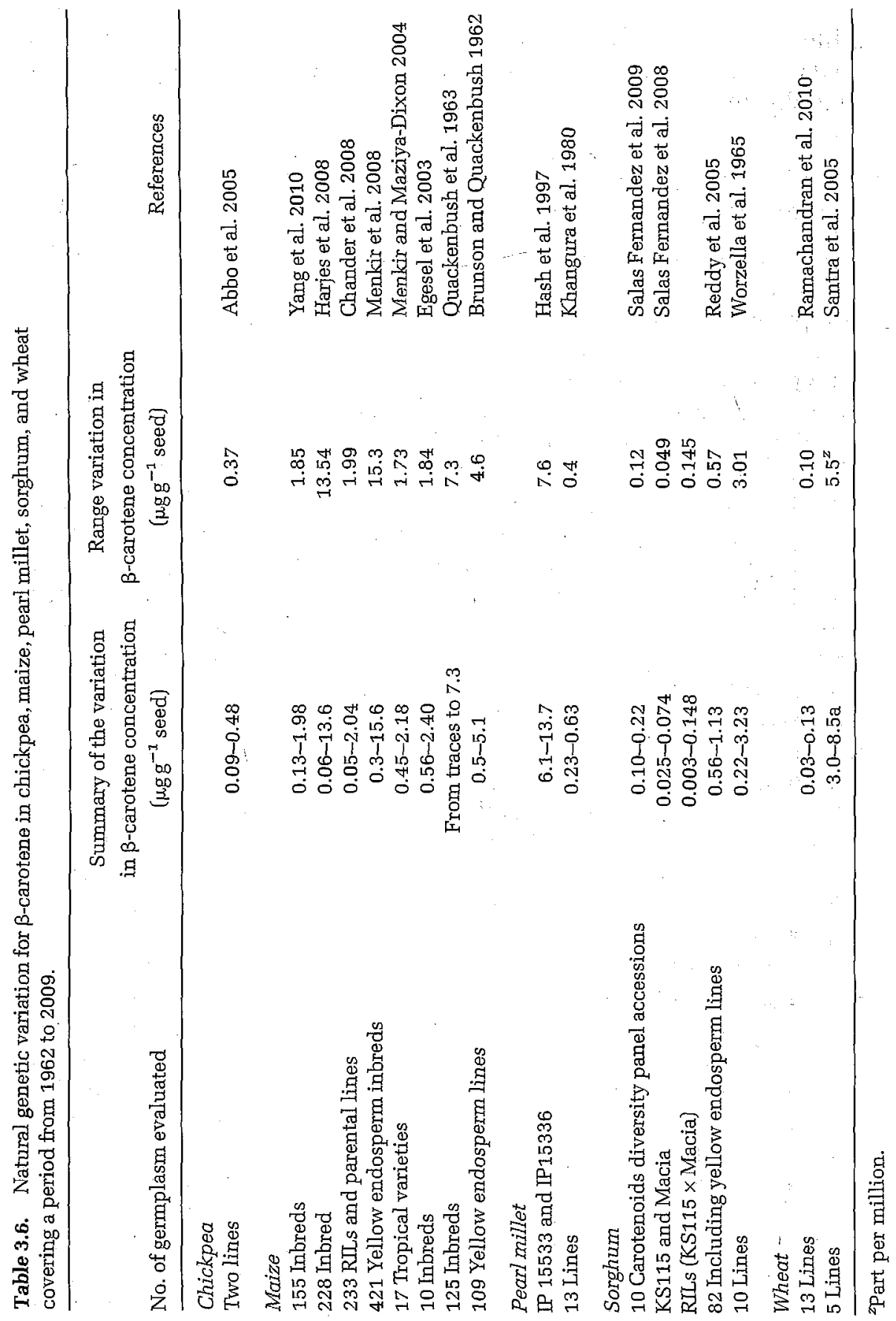


only $0.5-1.5 \mu g g^{-1} \beta$-carotene. Maize has been extensively studied for molecular polymorphism, mapped QTL, chemical pathways, and genes involved in carotenoids biosynthesis, including $\beta$-carotene (Table 3.7). Carotenes are intermediates in the carotenoids biosynthetic pathway and lycopene (the immediate precursor of provitamin A carotenes) represents a branch point in this pathway, which is further modified by lycopene $\beta$-cyclase and lycopene $\varepsilon$-cyclase (LCYE) enzymes that catalyze formation of terminal $\beta$ - and $\varepsilon$-rings, respectively, to form either $\beta$-carotene, $\alpha$-carotene, or $\beta$-cryptoxanthin. This pathway continues with hydroxylation of the carotenes that depletes the provitamin A pool by converting these compounds to nonprovitamin A xanthophylls. Pathway branching and hydroxylation are therefore key determinants in controlling vitamin A levels. Polymorphism at the LCYE locus in maize explained $58 \%$ of the variation in $\alpha$ - and $\beta$-carotene and a threefold difference in provitamin A compounds (Harjes et al. 2008), while a rare genetic variant, $\beta$-carotene hydroxylase 1 (crtRB1), increases $\beta$-carotene substantially in maize grains (Yan et al. 2010). Further, metabolite sorting of a germplasm collection identified 10 genetically diverse subsets representing biochemical extremes for maize kernel carotenoids and transcript profiling of this subset led to the discovery of the Hydroxylase 3 locus that coincidently mapped to a carotene QTL (Chander et al. 2008). The natural alleles at Hydroxylase 3 locus contribute $78 \%$ of variation and approximately 11 -fold differences in $\beta$-carotene relative to $\beta$-cryptoxanthin and $36 \%$ of the variation and fourfold difference in absolute levels of $\beta$-carotene (Harjes et al. 2008). The reduction in HYD3 transcripts leads to reduced conversion of $\beta$-carotene to downstream xanthophylls, causing $\beta$-carotene to accumulate (Vallabhaneni et al. 2009). Genetics tests such as the $H Y D 3$ assay (Vallabhaneni et al. 2009) together with the previously described LCYE assay (Harjes et al. 2008) may be used to select germplasm containing optimal HYD3 and $L C Y E$ alleles in breeding programs, which will lead to higher $\beta$-carotene levels in maize endosperm when both genes are highly expressed than either with optimal alleles of either gene alone. Furthermore, the experimental evidence from the association and linkage mapping reveals that $\operatorname{crtRB1}$ underlies a principal QTL associated with $\beta$-carotene concentration and conversion in maize kernels and crtRB1 alleles associated with reduced transcript expressions correlate well with higher $\beta$-carotene (Yan et al. 2010). The most favorable $\operatorname{crtRB1}$ alleles that are rare in frequency and unique to temperate germplasm are being introgressed via inexpensive PCRmarker-assisted selection into tropical maize germplasm adapted to developing countries. A program at CIMMYT has already achieved 


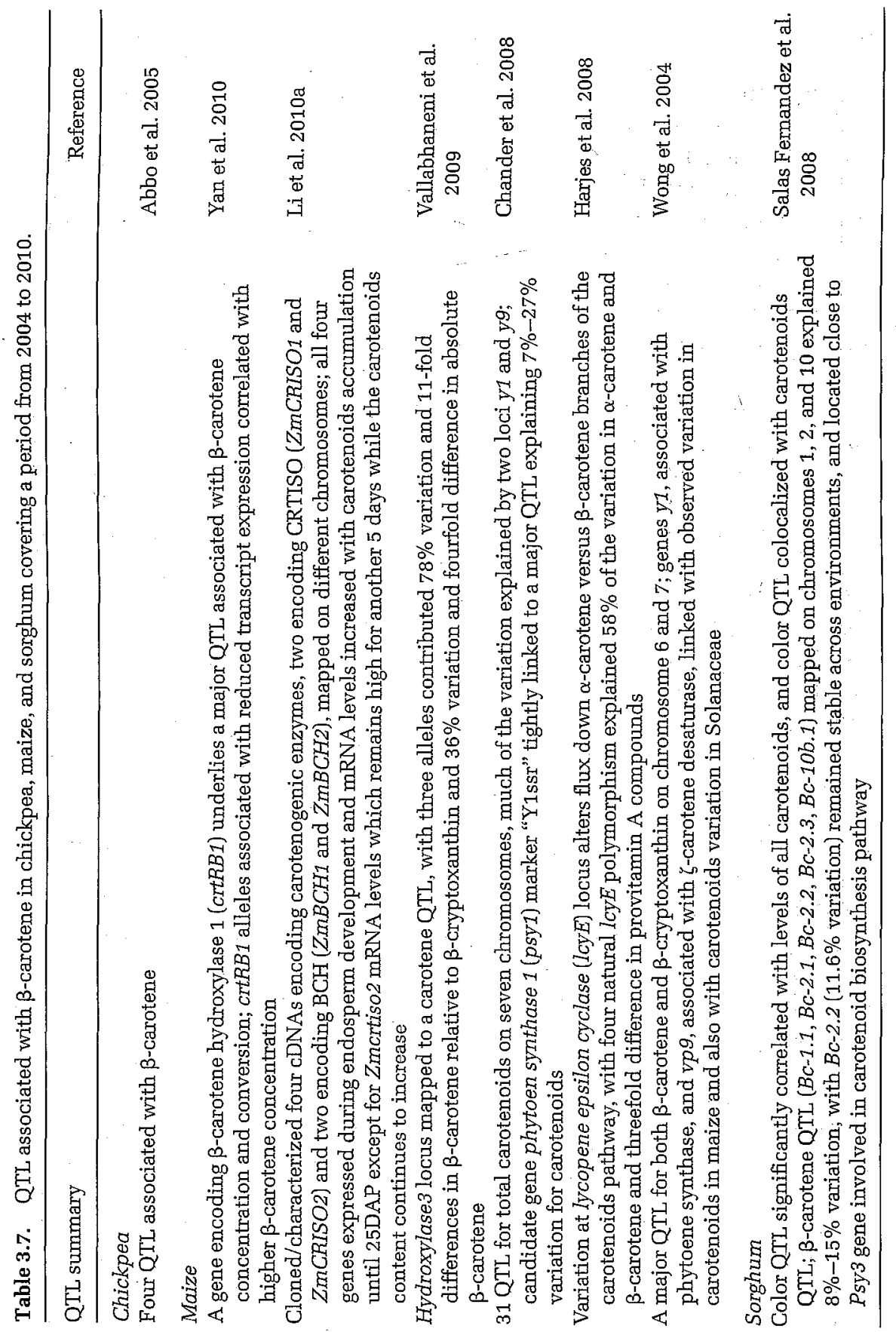


HarvestPlus provitamin A target concentrations by introgressing favorable $c r t R B 1$ and lcye alleles into tropical maize germplasm in a number of breeding lines, including a high $\beta$-carotene $\left(15 \mu \mathrm{g} \mathrm{g}^{-1}\right)$ temperate hybrid, CI7 $\times$ DEexp that contains the most favorable crtRB1 alleles. (Yan et al. 2010; Pixley et al. 2011b). Several hybrids with $\beta$-carotene concentration between 5 and $8 \mu \mathrm{gg}^{-1}$ and agronomically competitive with commercial hybrids were tested during summer 2009/2010 in Zambia and Zimbabwe, and the best hybrids will be further evaluated in Mexico, Zambia, and Zimbabwe prior to their release in these countries (Pixley et al, 2011b). Likewise, the International Institute of Tropical Agriculture (IITA), Ibadan, Nigeria, has introduced high $\beta$-carotene trait from temperate maize to tropical maize inbred lines, which ranged from $2.5 \mu \mathrm{g}^{-1}$ to $10.5 \mu \mathrm{g}^{-1}$, and hybrids involving some of these inbreds showed $25 \%-79 \%$ more provitamin A concentration than Oba Super II, a commercial yellow hybrids widely grown in Nigeria. The grain yield and agronomic traits of the best hybrids were comparable to those of Oba Super II (IITA annual report, 2009/2010; http:// annualreport.iita.org/ $\mathrm{p}=481$ ). The University of Illinois, USA has also reported some of the high provitamin $A$ lines of maize that include $A$ 619, C 17, DE 3, and SC 55 (see supplementary Table 1, Yan et al. 2010). The researchers at Vivekananda Parvatiya Krishi Anusandhan Sansthan (VPKAS), Almora, India and other affiliated Indian Council of Agricultural Research (ICAR) institutions in India have identified few promising maize inbred lines (CM 136 and CM 138, 08 HPLET-03-8 and 08 HPLET-03-41, NAI 125, BAJIM-8-10 and SE 547), with total carotenoids ranging from 20.2 to $56.1 \mu \mathrm{gg}^{-1}$ seed and $\beta$-carotene from 10.6 to $14.9 \mu \mathrm{gg}^{-1}$ seed (P.K. Agrawal, VKPAS, pers. commun.).

The 'Golden Rice 2' genetic stocks have been used to introgress high $\beta$-carotene trait into several Asian rice cultivars, both japonica and indica types, and it is expected that the products from such introgressions will soon be available for national release in Bangladesh, India, Indonesia, and the Philippines (Barry 2011).

Sorghum landraces have shown significant variation for carotenoids, with lutein, zeaxanthin, and $\beta$-carotene the predominant carotenoids. Yellow-endosperm color QTL in sorghum colocalized with carotenoid QTL, with major $\beta$-carotene QTL $B c-2.2$ found close to Psy3 gene, which is significantly associated with $\beta$-carotene concentration and endosperm color (Salas Fernandez et al. 2008). Furthermore, Salas Fernandez et al. (2009) reported that 164 yellow endosperm landraces from Niger and Nigeria clustered separately from the genotypes in a 68 individual diversity panel (Casa et al. 2008) with accessions differing in geographic origin and carotenoids content, which may provide 
additional source of genetic diversity to breed for $\beta$-carotene content in this crop.

The first step toward understanding how carotenoids are synthesized is to identify the enzymes involved and isolate the corresponding genes. Several maize complementary deoxyribonucleic acids (cDNAs) encoding carotenogenic enzymes have been cloned and genes identified: psy1, psy2, and psy3 (phytoene synthase), pds (phytoene desaturase), $z d s$ ( $\zeta$-carotene desaturase), lcyb (lycopene $\beta$-cyclase), and LCYE (Li et al. 2010a). Further, Li et al. (2010a) cloned and characterized four additional cDNAs, two each representing carotenoids isomerase (CRTISO) and $\beta$-carotene hydroxylase (BCH): Zmcrtiso1 and Zmcrtiso2 for CRTISO and Zmbch1 and Zmbch2 for BCH, mapped to different chromosomes. All four genes expressed during endosperm development and the coordinated up-regulation of Zmcrtiso1, Zmcrtiso2, Zmbch1, $\mathrm{Zmbch} 2$ until 25 days after pollination is consistent with the observed accumulation of carotenoids, although Zmcrtiso2 remained at high levels for the next 5 days while the carotenoids content continues to increase. The enzymes are highly conserved in sequence, expression, and activity, but subtle differences in the expression profiles of the CRTISO enzymes and the expression and activities of the $\mathrm{BCH}$ enzymes hint at divergent roles in plant carotenoids biosynthesis that may be useful in the development of more refined strategies to engineer carotenoids synthesis and composition in staple crops (Li et al. 2010a) (Fig. 3.1).

\section{E. Developing Seed Iron- and Zinc-Dense Cultivars Using Conventional Breeding and Genomic Tools and Cultivars Adoption}

1. Grain Minerals-Dense Cultivars and Hybrids. Unlike other approaches (such as fortification or supplementation), biofortification is a sustainable and cost-effective way to address malnutrition in developing countries. The main advantage with this approach is that once seed mineral-dense cultivars are developed, adapted for cultivation, and accepted by the end users, these will be grown by the farmers and the produce from such mineral-dense crops will be easily available to large communities at low cost-even in remote regions where other approaches may not succeed. Until recently, breeding efforts focused on raising productivity with hardly any thought to improving seed quality except for improving the protein quality in maize and wheat, and more particularly the essential seed-micronutrients such as $\mathrm{Fe}$ and Zn. Technological innovations including the use of dwarfing genes have 


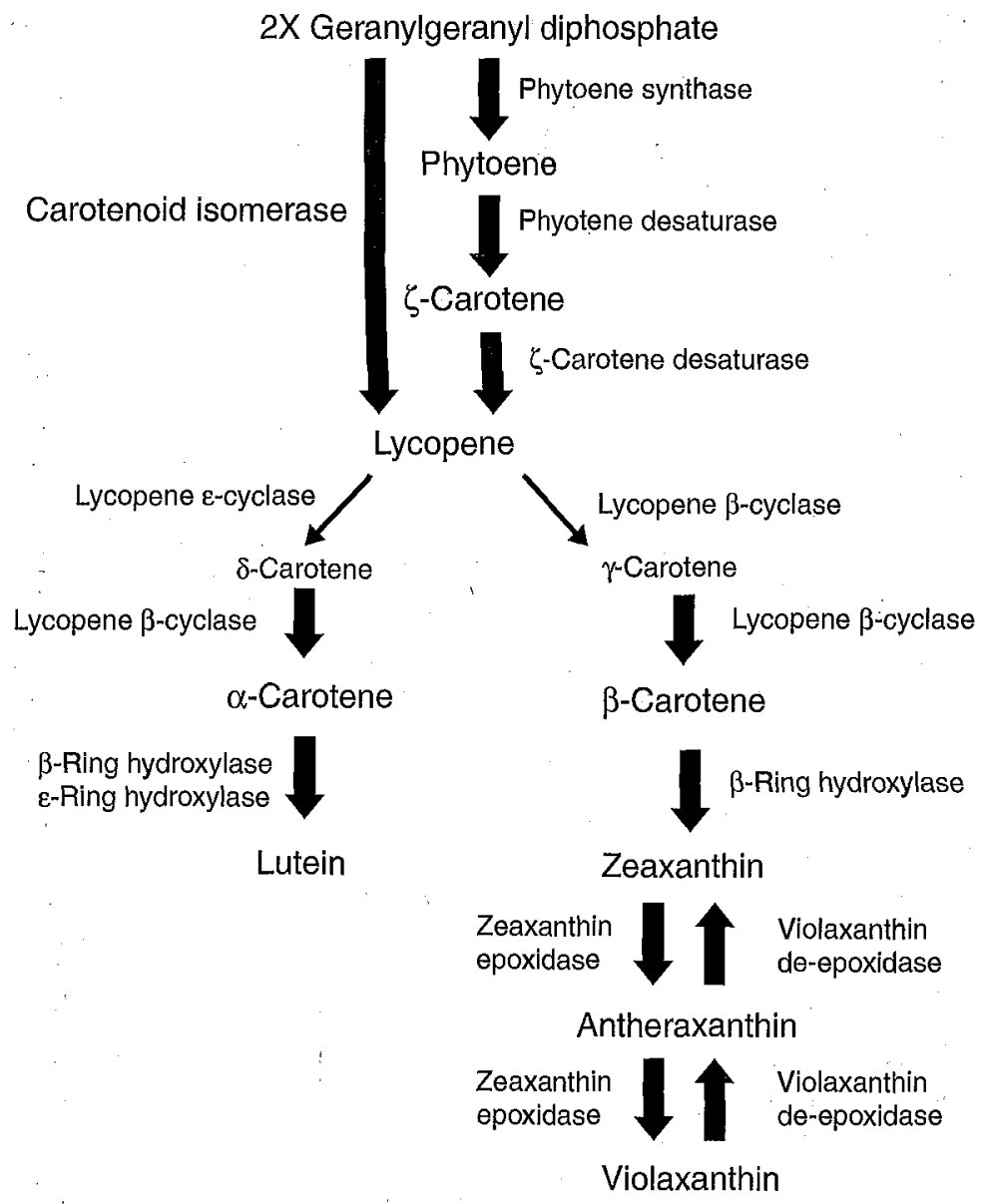

Fig. 3.1. Plant carotenoid biosynthesis pathway, (Adapted from Ramachandran et al. 2010).

boosted crop yields across continents; with the result that many countries are now more or less self-sufficient in food production. What is needed now is to improve the nutritional quality of staple food crops to provide nutritious foods to the poor who often consume diets poor in micronutrients. Agriculture research must therefore focus on both increasing production and improving diets in sustainable ways. Breakthroughs in plant breeding and nutritional genomics including transgenes (see Section VII.A) has simplified and hasten the development of seed mineral-dense cultivars to address rampant micronutrient malnutrition in developing countries. 
Breeding for seed mineral-dense cultivars is a recent activity (since mid-1995), mostly supported by the HarvestPlus Challenge Program, to develop $\beta$-carotene (precursor of vitamin A) rich and/or mineral-dense (largely Fe and Zn) cultivars of six major staple food crops (Phase I)common bean, cassava, maize, rice, sweet potato, and wheat. Ten additional crops (bananas/plantains, barley, cowpeas, peanut, lentil, millet, pigeon pea, potatoes, sorghum, and yam) were added in Phase II to improve seed mineral density (Pfeiffer and McClafferty 2007).

The proof of concept was demonstrated by CGIAR institutions, showing that high nutrient traits can be combined with superior agronomic characteristics into high yielding genetic backgrounds using conventional breeding, applied genomics, and/or transgene(s) technology. CGIAR institutions in partnership with National Agricultural Research System (NARS) have made considerable progress toward developing seed mineral (iron and/or zinc)-dense elite germplasm/cultivars in common bean, rice; and wheat (Table 3.8). For example, breeding at CIAT is directed to transfer high seed $\mathrm{Fe}$ and $\mathrm{Zn}$ traits into a range of commercial classes in both gene pools of common bean, that is, large-seeded Andean and small-seeded Mesoamerican beans. A number of donor lines with high seed Fe and $\mathrm{Zn}$ were crossed with adapted cultivars from different market and seed types and the progenies with high seed-Fe and -Zn concentrations are in various stages of evaluation. The variation observed in some of the progenies ranged from 76 to $154 \mathrm{ppm} \mathrm{Fe}$ and from 28 to $44 \mathrm{ppm} \mathrm{Zn,} \mathrm{with} \mathrm{some}$ progenies combining high $\mathrm{Fe}$ and $\mathrm{Zn}$ into improved genetic background. Micronutrient-dense bush and climbing bean cultivars with high yield potential have already been released in Bolivia, Colombia, and Zimbabwe (Blair et al. 2010d), and others are in prerelease stage in several Eastern African countries (e.g., Rwanda, DR Congo).

The first seed Fe-dense rice breeding line, IR68144-2B-2-2-3, has been released as Maligaya Special 13 (MS13) for cultivation in the Philippines (Sison et al. 2006). An improved version of the open pollinated pearl millet variety, ICTP 8203 , containing $~ 10 \%$ higher seed-Fe than the original ICTP 8203 background, has been identified for multilocational evaluation prior to its release in India, with adaptation to most of peninsular India. ICTP 8203 was produced by random mating five $\mathrm{S}_{2}$ progenies of an Iniari (early maturing) landrace originating from Northern Togo, and released as MP124 for cultivation in Maharashtra and Andhra Pradesh, India. It flowers in 50-52 days; the panicles are of medium length, compact to semicompact, and cylindrical to lanceolate with a slight tapering toward the tip; dark gray and large seed size (>12 g $1000^{-1}$ ); and resistance to downy mildew caused by Sclerospora 
Table 3.8. Seed-iron ( $\mathrm{Fe}$ ) and/or -zinc ( $\mathrm{Zn}$ ) dense advanced breeding lines/cultivars developed in common bean, pearl millet, rice, and wheat covering a period from 2003 to 2010 .

Summary of the seed-Fe and/or - Zn dense lines

developed by conventional crossing and selection

References

Seed-Fe concentration

Common bean

NUA35 and NUA56 showed on average 18 and $23 \mathrm{mg} \mathrm{kg}^{-1}$

higher Fe than control cultivar 'CAL96' ( $58 \mathrm{mg} \mathrm{kg}^{-1}$ ),

when evaluated over 15 sites with diverse agroecologies

in Bolivia, Colombia, Costa Rica, and Guatemala

Pearl millet

Improved versions of open pollinated variety (ICTP 8203)

outyielded original ICTP 8203 bulk (2874 $\left.\mathrm{kg} \mathrm{ha}^{-1}\right)$

ICRISAT 2010

by $7 \%-10 \%$, with $7 \%-18 \%$ more $\mathrm{Fe}\left(94-103 \mathrm{mg} \mathrm{kg}^{-1}\right.$ )

Rice and $5 \%-15 \%$ more $\mathrm{Zn}\left(63-69 \mathrm{mg} \mathrm{kg}^{-1}\right)$

An seed-Fe dense line, IR68144-2B-2-2-3, released as Maligaya Special 13 (MS13) in Philippines

Wheat

A synthetic line, 68.111/RGB-4//WARD/3/FGO/4/

$\mathrm{RABI} / 5 / \mathrm{Ae}$. Sq. (878), showed 25\% more Fe than controls

Backcross-derived lines involving $A$. kotschyi showed $67 \%-116 \%$ increase in Fe than control, WL711 (22 $\mathrm{mg} \mathrm{kg}^{-1}$ seed)

Amphidiploids involving $A$. kotschyi accessions showed $97 \%-117 \%$ and $68 \%-111 \%$, respectively, higher $\mathrm{Fe}$ in seed and flag leaf than controls

\section{Seed-Zn concentration}

\section{Common bean}

Progenies involving Perola $\times$ Guapo Brilhante or TPS Nobre $\times$ Guapo Brilhante recorded $37 \%$ increase in $\mathrm{Zn}$ content

NUA35 and NUA56 showed on average 8 and $7 \mathrm{mg} \mathrm{kg}^{-1}$ higher zinc than control cultivar 'CAL96' ( $26 \mathrm{mg} \mathrm{kg}^{-1}$ ), when evaluated over 15 sites with diverse agroecologies in Bolivia, Colombia, Costa Rica, and Guatemala

Progenies with $10 \%$ increase in $\mathrm{Zn}$ content developed Wheat

A synthetic line, 68.111/RGB-4//WARD/3/FGO/4/RABI/ 5/Ae. Sq. (878), showed $25 \%$ more Zn than controls

Backcross-derived lines involving $A$. kotschyi showed $75 \%-133 \%$ increase in $\mathrm{Zn}$ than control, WL711 (20 $\mathrm{mg} \mathrm{kg}^{-1}$ seed)

Amphidiploids involving $A$. kotschyi accessions showed, respectively, $67 \%-139 \%$ and $54 \%-91 \%$ higher $\mathrm{Zn}$

- in seed and flag leaf than controls

de Rosa et al. 2010

Blair et al. 2010d

Gelin et al. 2007

Calderini and OrtizMonasterio 2003 Tiwari et al. 2010

Rawat et al. 2009a 
graminicola (Rai et al. 1990). Further work at ICRISAT is in progress to identify genetically diverse germplasm (noniniary type) from the recently constituted pearl millet mini-core collection (Upadhyaya et al. 2010) for developing hybrid parental lines with high seed-Fe concentration. In a two-rowed barley, the pooling of three most favourable QTL alleles increased seed-Zn content and concentration by an average of $53 \%$ and $75 \%$, respectively (Lonergan et al. 2009).

The AgroSalud project (www.AgroSalud.org), a consortium of centers from the CGIAR, NARS, Universities, and NGOs formed in 2005 with funding support from Canadian International Development Agency (CIDA), aims to improve food and nutrition security among vulnerable populations living in 14 Latin American and Caribbean countries through nutritionally enhanced biofortified crops (beans, maize, rice, sweet potato) and food products derived from these crops and cassava. To date, AgroSalud have commercially released 21 maize cultivars with higher tryptophan and lysine levels (protein quality not the subject of this review) in Bolivia, Colombia, El Salvador, Guatemala, Haiti, Honduras, Mexico, Nicaragua, and Panama; eight rice cultivars with higher iron in Bolivia, Cuba, and Panama; five bean cultivars with higher iron in Bolivia, Brazil, Cuba, and Guatemala; and eight sweet potato cultivars with more $\beta$-carotene in Brazil, Cuba, Dominican Republic, Haiti, and Peru. An additional 10 nutritionally enhanced cultivars are in the pipeline for release in the region. More importantly, the project partners in Cuba, Nicaragua, and Panama have succeeded in influencing the policy makers to include biofortification as a core breeding activity for improving the nutritional quality of these crops (http://www.AgroSalud.org/descargas/ AgroSalud_consolidated\%20final_report_2010_ene18_11.pdf).

More recently, Glahn and Hoekenga (2011) detected three QTL with large effects for bioavailable iron in maize that they integrated into new cultivars of maize highly similar to each other but containing different QTL for iron bioavailability. Further, the feeding trials demonstrated that QTL associated with high bioavailable iron in maize provided approximately twice as much iron to the birds relative to the low bioavailable iron lines.

Wild and weedy or uncommonly cultivated relatives of common bean and wheat have shown large variability for seed-Feand -Zn concentrations (see Section V.A). Mineral nutrient concentrations in the perennial lines derived from an interspecific cross involving a tall wheatgrass (Thinopyrum elongatum) and bread wheat revealed $44 \%, 40 \%, 24 \%, 23 \%, 32 \%$, $30 \%$, and $30 \%$ higher than control cultivars for calcium, copper, iron, magnesium, manganese, phosphorus, andzinc,respectively(Murphyetal. 2009). Interspecific crosses involving wild emmer and Aegilops species 
with cultivated wheat's produced seed mineral (Feand Zn)-dense progenies (Rawatetal. 2009b; Neelam etal.2010a; Tiwari etal. 2010). For example, backcross progenies involving Aegilops peregrina $\left(2 n=28, U^{\mathrm{P}} \mathrm{U}^{\mathrm{P}} \mathrm{S}^{\mathrm{P}} \mathrm{S}^{\mathrm{P}}\right)$ with T. aestivum showed significantly higher seed micronutrients (nearly $100 \%$ increase in seed $\mathrm{Fe}$ and more than $200 \%$ increase in seed $\mathrm{Zn}$ ), high thousand-seed weight, and harvest index. The seed-Fe concentration in wheat cultivars was $\sim 28 \mathrm{mg} \mathrm{kg}^{-1}$ while zinc $\sim 22 \mathrm{mg} \mathrm{kg}^{-1}$ seed. The in situ hybridization and marker analysis of these progenies further revealed that chromosomes 4 and 7 of Ae. peregrina carry the genes for high seed-Fe and -Zn concentrations. Likewise, CIAT conducted interspecific backcrosses andrecurrentselection totransferhigh seed-Feand-Zn from Phaseoluscoccineus, $P$. dumosus, and $P$. acutifolius into common bean. Some interspecific progenies showed $30 \%-40 \%$ increases in seed Fe over the control cultivars (Acosta-Gallegos etal. 2007; SteveBeebe, CIAT, pers. commun. 2011).

Marker-assisted backcrossing' (MABC) has been successfully employed to transfer the low-phytate trait into improved genetic backgrounds in soybean. Landau-Ellis and Pantalone (2009) used SSRs (Satt237 on LGN and Satt561 on LG L) (Walker et al. 2006) to transfer the low-phytate trait from CX 1834-1-2, which is controlled by two recessive genes (Oltmans et al. 2004), into an improved genetic background, and by $\mathrm{BC}_{4}$ generation, they fully recovered the recurrent parent genome of 5601T (soybean cultivar of maturity group V) while simultaneously confirming the presence of both low-phytate loci in backcross progenies. Evidence suggests, therefore, that the MABC approach is an effective breeding method to transfer low-phytate trait into improved genetic background.

\section{Adoption of Biofortified Cultivars by Farmers. Adoption studies} conducted under AgroSalud project suggest high acceptance of nutritionally enhanced cultivars of maize (tryptophane) in El Salvador, beans $(\mathrm{Fe})$ in Cuba and Nicaragua, sweet potato ( $\beta$-carotene) in Nicaragua, and rice $(\mathrm{Fe}$ ) in Bolivia and Panama (www.AgroSalud.org/descargas/poster \%20agrosalud_ingles_v2_impresion_oct10.pdf). The pattern of early diffusion and adoption of $\beta$-carotene-rich cassava cultivars (Fukuda et al. 2008) in northeast Brazil revealed large gap between actual early adoption in some regions $(62.5 \%$ vs. $15 \%)$ in comparison with the potential adoption (62\% and 64\%), which Gonzálezet al, (2011) relate it with public awareness of the new cultivars advantages, public entities as the main information sources, and farmers participation in early stages of evaluation. This study also revealed the lack of seed availability as one of the main factors limiting the adoption process and thus, suggested strengthening seed production and distribution for better 
diffusion of the newly developed cultivars among the producers (González et al. 2011). Preliminary studies in Kenya, Mozambique, and South Africa have shown that provitamin A biofortified maize (grain color orange yellow because of $\beta$-carotene) may be adopted where white-seeded maize is the predominant staple crop (Wolson 2007; Stevens and Winter-Nelson 2008).

QPM is the first biofortified crop, released and disseminated in East Africa. The adoption of QPM varied greatly in Ethiopia, Kenya, Tanzania, and Uganda, from none in Kenya to more than half of the farmers growing QPM in Uganda. Further, analysis of the pattern of adoption of QPM in these countries revealed that agronomic performance of QPM varieties; postharvest processing; the cooking and sensory characteristics (taste and flavor); understanding QPM's nutritional benefits; higher participation of the farmers in QPM evaluation; and reliable and continuous supply of seeds, all significantly impacted the adoption of QPM in Tanzania and Uganda (Hugo de Groote, pers. commun., GIMMYT). A QPM cultivar, Obatanpa, first released in Ghana in 1992, is now commercially grown in 15 other African countries (Krivanek et al. 2007).

A range of biofortified crops ( $\mathrm{Fe}, \mathrm{Zn}$, and/or $\beta$-carotene) are being developed, many in pipeline, which will soon be available to farmers for cultivation that will provide opportunities to the social/economic personal to assess adoption and to nutritionists to assess the impact of these cultivars on human nutrition leading to improved health in regions prone to micronutrient malnutrition.

\section{F. Breeding Issues Associated with Selecting Seed Mineral-Dense Progenies}

Mineral concentration in a plant's shoot is dependent on the rate of dry matter accumulation and micronutrients absorption. When dry-matter accumulation increases at a faster rate than micronutrient accumulation, a so-called "dilution effect" can be observed, whereas increased micronutrient concentrations will result when micronutrient accumulation increases at a faster rate than dry matter accumulation ("synergism effect") (Jarrell and Beverly 1981). It is a well-known fact that when breeders select for one-resource using trait, such as yield, less resources remain for other resource-using functions, that is, trade-offs between seed size and seed number, yield and resistance to pest and diseases, or yield and nutrition (Davis 2005, 2009; Morris and Sands 2006). The extent of such trade-offs is a question that must be addressed.

Modern plant breeding has revolutionized agriculture, resulting in several fold increases in production and productivity of most staple 
crops that form the major basis for human diets. To achieve higher productivity, plant breeders have concentrated mainly on traits that raise yield per se. The so-called "green revolution" has raised productivity, but did not concentrate on producing food that was as nutritious and tasty as from the traditional cultivars/landraces. For example, declines are reported in the mineral density of broccoli (Farnham et al. 2000) and wheat (Garvin et al. 2006; Fan et al. 2008; Murphy et al. 2008), and in protein concentration of maize (Scott et al. 2006). The evidence for these crops clearly shows uniformly inverse associations between seed yield and nutrition, indicating that genetic dilution effects (trade-offs) may be common when selective breeding successfully increases crop yields (Davis 2009).

Furthermore, nutritious germplasm/cultivars can be more vulnerable to pests and diseases (Arnason et al. 1993, Morris and Sands 2006). The environmental trade-offs between yield and nutritional quality could result either from the variation in soil health or quality (nutrientdeficient soils or soils affected by salinity/alkalinity or acidity; such soil conditions lead to nutrient imbalance in the soil) and soil fertility related factors (i.e., NPK effect on seed composition) or due to drought and high temperature during the seed development. For example, reduced seed protein and minerals and altered lipid composition have been reported in barley, potato, rice, and soybean as a consequence of nitrogenous fertilizer application (Riedel 2010) or global climatic changes, especially due to high temperature and high $\mathrm{CO}_{2}$ concentrations in the environments (Högy and Fangmeier 2008; Taub and Wang 2008; Taub et al. 2008; Pleijel and Danielsson 2009; Sinha et al. 2009; DaMatta et al. 2010; Erbs et al. 2010). The challenge for the agricultural research community is to minimize any possible negative trade-offs between yield and nutrient concentrations, to provide nutritious staple foods for growing populations (Davis 2009).

How much seed phytate can be reduced (either through mutagenesis, or conventional crossing, and selection or by using transgenes) without adverse effect on seed germination and plant development is an open question. Research to date suggest that it is possible to breed firstgeneration low-phytate (50\%-95\% reduction) hybrids/cultivars in maize, barley, rice, and soybean, which performed relatively better than the original low-phytate mutants derived from the ưse of mutagens (Raboy et al. 2001; Raboy 2002; Spear and Fehr 2007); however, it is yet to be investigated whether low-phytate seeds remain beneficial to human health?

-In conclusion, few QTLs with major effects on seed Fe, Zn, and phytate have been reported in common bean. A major QTL for IRA 
under Fe-deficient and another major QTL for IRA under Fe-sufficient conditions have been identified, the latter associated with several QTL for seed-Fe concentration. A major locus from wild emmer wheat, GpbcB1, mapped as a single Mendelian locus encodes a NAC transcription factor, TtNAM-B1, has been cloned, which accelerates senescence and increases seed protein, $\mathrm{Fe}$, and $\mathrm{Zn}$. Interspecific progenies containing two Fe QTL and one Zn QTL from Aegilops species showed exceptionally high seed-Fe and -Zn concentrations. Yellow endosperm color QTL in sorghum is colocalized with carotenoids QTL, with major $\beta$-carotene QTL $B C$-2.2 found close to Psy3 gene, which is significantly associated with $\beta$-carotene concentration and endosperm color.

AgroSalud project has released several maize cultivars with high tryptophan and lysine; beans and rice with high seed $\mathrm{Fe}$; and $\beta$-carotene-rich sweet potato have been released for cultivation in several Latin American countries. CIAT has released seed micronutrient-dense ( $\mathrm{Fe}$ and $\mathrm{Zn}$ ) common bean in Bolivia, Colombia, and Zimbabwe; with many in prerelease stage in several Eastern African countries including those: in Rwanda and DR Congo. In Asia, a seed iron-dense rice cultivar has been released for cultivation in the Philippines, while an improved version of an open pollinated variety with high seed iron, ICTP 8203 , is in the prerelease stage in India.

CIMMYT and IITA researchers have identified $\beta$-carotene-rich maize germplasm and transferred high $\beta$-carotene trait into improved genetic background. The selected lines are under multilocational evaluation prior to their release in Mexico and in Africa. At the CIMMYT, molecular markers for $L y c E$ and $H y d B$ have accelerated breeding by one season, and have substantially enhanced efficiency and effectiveness of high-provitamin A maize breeding. Marker-assisted selection has been successfully employed to transfer low phytate into improved soybean cultivars.

Limited studies on cultivar adoption suggest high adoption of nutritionally enhanced crops in some countries in Latin America, which brought a paradigm shift to include biofortification as core breeding activity to enhance the nutritional quality of these crops in Cuba, Nicaragua, and Panama.

\section{ENHANCING SEED IRON, ZINC, AND B-CAROTENE USING TRANSGENE(S)}

\section{A. Transgenes for Nutritional Enhancement of Food Crops}

Targeted improvement of mineral nutrition through plant biotechnology may be another more sustainable albeit sometimes controversial 
approach to combat widespread deficiencies in human populations, particularly in developing world (Zimmerman and Hurrel 2002; Bouis 2007; Hirschi 2009). Few genes have been identified that either associate with increased $\mathrm{Fe}$ and $\mathrm{Zn}$ uptake/accumulation in developing seeds or reduced phytate such as the enzyme phytase that degrades phytate and thus releases more bioavailable Fe and Zn to absorption. The biosynthetic pathway to carotenoids synthesis and genes associated with accumulation of carotenoids has been characterized in several related species (Misawa et al. 1990; Zhu et al. 2007; Lu and Li 2008). We summarize below the update on use of transgene(s) to enhancing seed $\mathrm{Fe}, \mathrm{Zn}$, and/or $\beta$-carotene or reducing phytate concentrations in maize and rice, two of the three major cereal seeds, and soybean, an oil crop but rich source of protein.

1. Iron and Zinc. Ferritin is the iron storage protein found in animals, plants, and bacteria, which can carry up to 4,500 iron atoms in a central cavity (Theil 1987). The ferritin protein takes up Fe, stores it in a nontoxic form, and releases it when needed for metabolic functions. The ferritin iron represents a form of Fe that is highly bioavailable to humans. Plant ferritins are the products of a small gene family. Plant ferritin genes have been obtained from common bean, cowpea, lentil, maize, pea, and soybean (Prasad and Nirupa 2007). The expression of ferritin gene either from soybean or common bean, driven by the endosperm-specific promoters, have led to two- to threefold higher seed-Fe and - Zn levels in transgenic brown and/or polished seeds than WT rice (Table 3.9). Ferritins are the major players in plant $\mathrm{Fe}$ homeostasis. However, the use of ferritin as a biotechnology target to enrich seeds with $\mathrm{Fe}$ has met with limited success (only 1.5- to 3-fold increase compared to WT), probably due to a posttranscriptional regulation of the seed ferritin protein during fruit maturation.

A recent study in Arabidopsis reveals that there is no specificity for metal loading into the fruit, and that the step controlling metal loading into the seed occurs most likely by the regulation of hull to seed metal transport. Thus, the success of ferritin overexpression strategies for $\mathrm{Fe}$ biofortification would strongly benefit from the identification and engineering of mechanisms enabling the translocation of high amounts of Fe into the seed (Ravet et al. 2009).

In this regard, another gene for Nicotianamine synthase (NAS), a chelator of metals, is ubiquitous in higher plants and is a key component of their metal assimilation and homeostasis. Manipulation of cellular nicotianamine (NA) concentrations is a possible approach to improving Fe concentrations in planta (Douchkov et al. 2005). Overexpressing 
Table 3.9. Examples of transgenic rice with enhanced seed-iron (Fe) and -zinc ( $\mathrm{Zn}$ ) concentrations covering a period from 1999 to 2010.

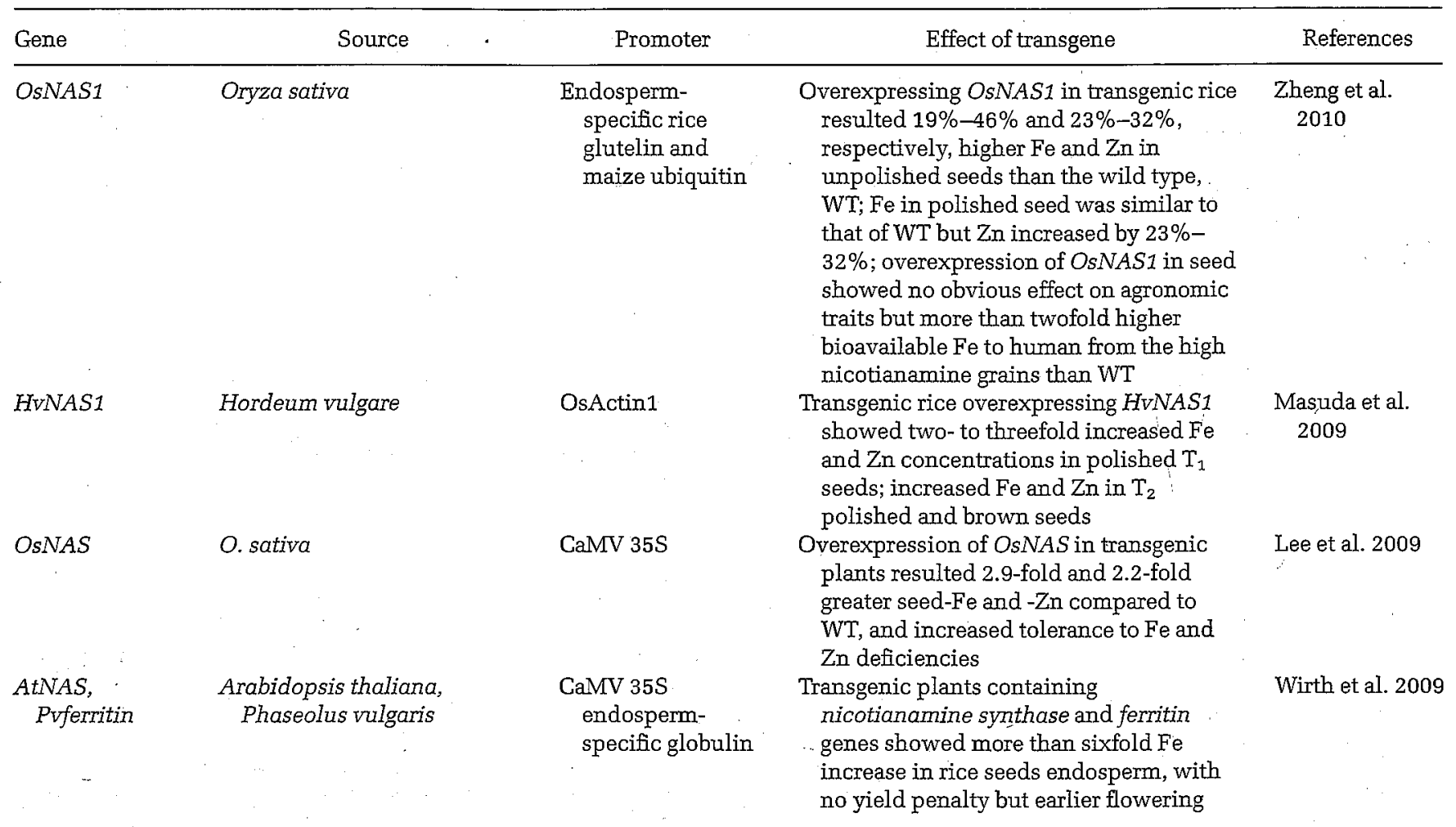




Mugeneic
acid (MA)

\section{Gmferritin Glycine $\max$}

SoyferH-1 G. $\max$

\section{Gmferritin G. $\max$}

$\begin{array}{lc}\text { Pvferritin } & \text { P. vulgaris, Aspergillus } \\ & \text { fumigatus } \\ \text { Pvpfe, } & \text { P. vulgaris, A. fumigatus, } O . \\ \text { Afphytase, } & \text { sativa }\end{array}$

$\operatorname{rgMT}$

Gmferritin

G. $\operatorname{miax}$
Endospermspecific glutelin

Endospermspecific glutelin, GluB-1, and globulin, Glb-1

\section{Endosperm-} specific glutelin

Endospermspecific glutelin

Endospermspecific glutelin

Endospermspecific glutelin, GluB-1
Transgenic rice containing IDS3 ( $H V N A A T-$ $B$ ) gene had up to 1.4 and 1.35 times higher seed-Fe and - Zn concentrations, respectively, compared to WT

Transgenic rice cultivar, BRR 29, accumulated as much as $9.2 \mathrm{mg} \mathrm{kg}^{-1}$ iron in the seed than the WT

$$
\text { (3.8 } \mathrm{mg} \mathrm{kg}^{-1} \text { ) }
$$

Transgenic rice containing double ferritin (GluB-1/SoyferH-1 and Glb-1/SoyferH1) and single ferritin (Glb-1/Soyfer $H-1$ ) genes showed significantly higher seedFe (15.4-15.7 $\mathrm{\mu gg}^{-1}$ ), about 40\% higher than WT (11.2 $\mathrm{ggg}^{-1}$ )

Enhanced $\mathrm{Fe}$ and $\mathrm{Zn}$, both in brown and polished rice, in transgenic indica rice IR68144

Twofold increase in Fe content of the seeds from the transgenic rice, Taipei 309

Transgenic rice containing $p$ fe and phytase gene not only raised seed $\mathrm{Fe}$ (twofold) but also improved bioavailable Fe due to many fold increase in phytase activity which degrade phytate to release more bioavailable $\mathrm{Fe}$; overexpressing rgMT increased the cysteine content, which further enhanced bioavailable $\mathrm{Fe}$

Transgenic rice containing ferritin accumulated up to threefold more seed$\mathrm{Fe}\left(38.1 \mu \mathrm{g} \mathrm{g}^{-1}\right)$ than the WT $\left(11.2 \mu \mathrm{gg}^{-1}\right)$
Masuda et al. 2008

Khalekuzzaman et al. 2006

Qu et al. 2005

Vasconcelos et al. 2003

Lucca et al. 2002

Lucca et al. 2001

Goto et al. 1999 
NAS gene from barley and rice increased $\mathrm{Fe}$ and $\mathrm{Zn}$ concentrations by two- to threefolds in transgenic rice compared to WT, with no obvious effect on agronomic traits (Table 3.9): Using Caco-2 cell digest model, Zheng et al. (2010) further demonstrated that elevated NA concentration led to more than twofold higher bioavailable Fe from the high NA seeds than the WT. Thus, NA is a novel and effective promoter of Fe utilization. More importantly, the transgenic rice plants containing AtNAS and $P$ vferritin genes increased seed-Fe concentration by sixfold, with no yield penalty but such plants were earlier to flower, and that the $\mathrm{Fe}$ in the endosperm of the transgenic rice lines accumulated in spots, most probably as a consequence of spatially restricted ferritin accumulation (Wirth et al. 2009). Biofortifying rice with NAS alone or in combination with ferritin has great potential in combating global human Fe deficiency in people dependent on rice for their sustenance (Lee et al. 2009; Zheng et al. 2010).

Mugineic acid family phytosiderophores (MAs) play an important role in the uptake of $\mathrm{Fe}$ from the soil and $\mathrm{Fe}$ transport within the plant in graminaceous plants. NA is the precursor of MAs that are natural Fe(III) chelators for Fe acquisition from the rhizosphere (Mihashi and Mori 1989; Takagi 1976). Rice produces DMA that chelates Fe(III) and contributes to Fe uptake and internal transport (Kobayashi and Nishizawa 2008). By hypothesizing that overexpression of the NAS in rice would enhance the synthesis of NA and DMA, and thus increase Fe and Zn concentrations in the seeds, Masuda et al. (2009) inserted HVNAS1 into rice and the resultant transgenic plants showed increased HvNAS1 expression, endogenous NA, and phytosiderophore content in shoots, roots, and seeds. They detected two- to threefold increases in Fe and Zn concentrations in transgenic polished $\mathrm{T}_{1}$ seeds, with both the elements (NA and DMA) also high in polished and brown $\mathrm{T}_{2}$ seeds, which clearly indicate overproduction of NA enhances the translocation of $\mathrm{Fe}$ and $\mathrm{Zn}$ into rice seeds.

2. Phytate. Alternative to mutagenesis is to either block the phytate biosynthetic pathway or degrade phytate in developing seeds to produce low-phytate crops through transgene. Phytases, known to enhance phosphate and mineral uptake in monogastric animals, catalyze the hydrolysis of phytate to myo-inositol pentakisphosphate $\left(\mathrm{IP}_{5}\right)$ or to less phosphorylated myo-inositol phosphates IP3. The phytases isolated from plant, microorganism, and animal tissues are broadly classified into three types, 3-phytases, 6-phytases, and 5-phytases, depending on the initiation site of dephosphorylation (Rao et al. 2009). A thermotolerant phytase gene from Aspergillus has been used to alter phytic acid in maize, rice, and soybean (Table 3.10). For example, transgenic 


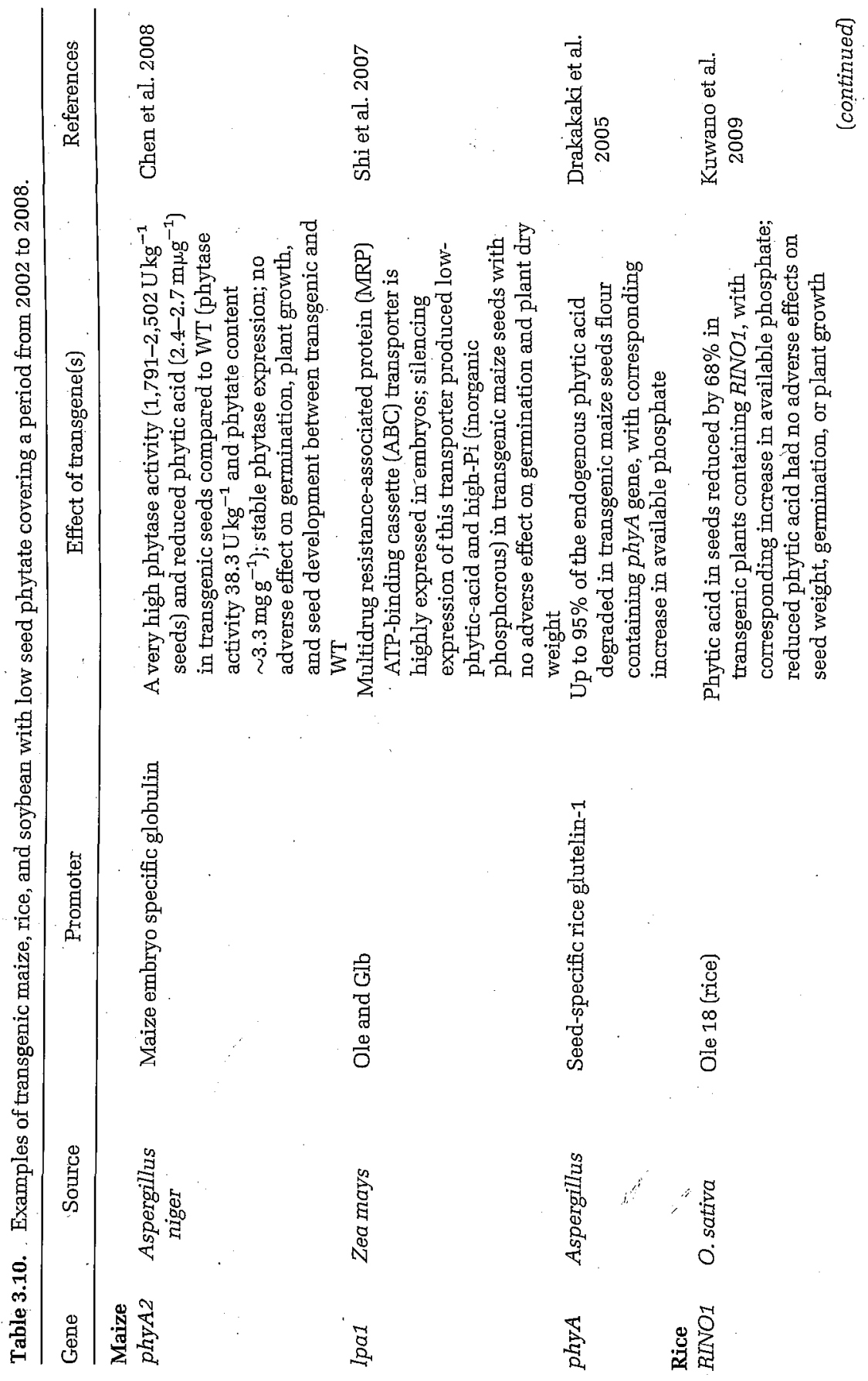




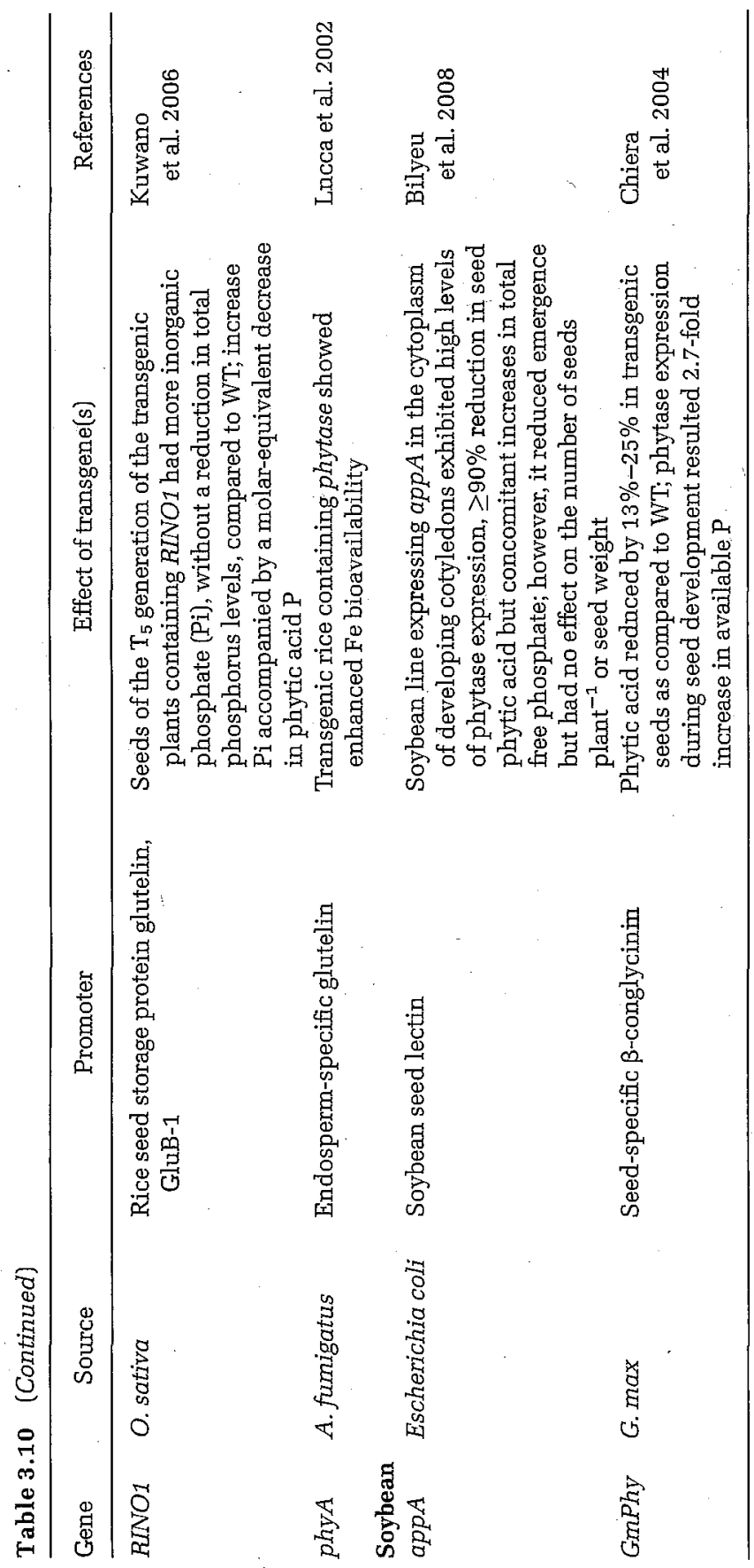


maize containing phyA2 gene showed a very high phytase activity (1791-2502 Unit $\mathrm{kg}^{-1}$ seed) and reduced phytic acid compared to WT (Chen et al, 2008).

Meanwhile, a MRP ATP-binding cassette transporter is highly expressed in embryo, and by silencing expression of this transporter, Shi et al. (2007) produced transgenic maize low in seed phytate but high in $\mathrm{Pi}$. The rice Ins(3) $\mathrm{P}_{1}$ synthase gene (RINO1) is highly expressed in developing seed embryos and in the aleurone layer, where phytic acid is synthesized and stored. Targeted insertion of RINO1 in rice resulted substantial reduction in phytic acid, with corresponding increase in available Pi (Kuwano et al. 2009). In all these cases, seed-specific promoters were used and the resultant transgene(s) had no adverse effect on seed germination, plant growth and development, unlike lp mutants generated through induced mutagenesis, which are affected by poor germination and emergence as well as reduced seed weight (see Section V.A.2). The molecular approaches may be more advantageous than mutagenesis combined with traditional breeding to manipulate phytic acid biosynthesis in food crops (Kuwano et al. 2006; Blair et al. 2008).

3. $\beta$-Carotene. Transgenic approaches have been used to effectively modify the carotenoids concentration to enhance nutritional value of maize and rice (Table 3.11). For example, Ye et al. (2000) were the first to introduce the entire $\beta$-carotene biosynthetic pathway to produce transgenic rice with yellow endosperm grains containing $\sim 1.6 \mu \mathrm{gg}^{-1}$ carotenoid, and coined the term 'Golden Rice'. Subsequent development led to creation of 'Golden Rice 2' containing exceptionally high amount of carotenoids (maximum $37 \mu \mathrm{gg}^{-1}$ ), of which $\beta$-carotene was the predominant (31 $\mathrm{gg} \mathrm{g}^{-1}$ ) (Paine et al. 2005). The proof of concept has been demonstrated that genes psy and crtI or psy, crtI and lycopene $\beta$-cyclase under endosperm-specific promoters have been effective for elevating $\beta$-carotene concentration in transgenic rice, with more $\beta$-carotene in polished grains. More importantly, the incorporation of genes for carotenogenesis in seeds by transgenesis or by introgression did not change any significant agronomic characteristics in rice plants (Paine et al. 2005; Baisakh et al. 2006; Datta et al. 2006, 2007).

Other researchers have used similar strategy as adapted for the creation of 'Golden Rice 2' to alter carotenoids profiles of the maize seeds (Table 3.11). Overexpression of bacterial genes $\operatorname{crtB}$ and $\operatorname{crtl}$, under the control of endosperm-specific promoter "super $\gamma$-zein," resulted in an increase of total carotenoids of up to 34-fold with preferential accumulation of $\beta$-carotene in the maize endosperm (Aluru 
Table 3.11. Examples of transgenic maize and rice with enhanced $\beta$-carotene in the seeds covering a period from 2000 to 2009.

Gene Source Promoter Effect of transgene References

\section{Maize}

psy1

Z. mays

Wheat LMW glutenin

$\begin{array}{ll}\text { crtI } & \text { Pantoea ananatis } \\ \text { dhar } & \text { O. sativa } \\ \text { foIE } & \text { E. coli } \\ \text { psy1 } & \text { Z. mays }\end{array}$

crtI

\section{P. ananatis}

Lycopene $\beta$-cyclase $b c h$

Gentiana lutea

G. Iutea

crtW

Paracoccus

Barley D-hordein

Barley D-hordein

Barley D-hordein

Endosperm-specific wheat glutenin

Endosperm-specific

barley hordein

Endosperm-specific rice prolamin

Endosperm-specific rice glutelin-1

Endosperm-specific maize $\gamma$-zein
Transgenic maize containing psy1, crtI, dhar, and folE gene contains 169-fold the normal amount of $\beta$-carotene, sixfold the normal amount of ascorbate, and double the normal amount of folate, and the levels of engineered vitamins remained stable at least through to the $\mathrm{T}_{3}$ homozygous generation

A South African white maize, M37W, transformed with five carotenogenic genes controlled by different endosperm-specific promoters; transgenic plants expressing different enzyme combinations and showing distinct metabolic . phenotypes recovered, with some accumulating maximum of $57.35 \mu \mathrm{gg}^{-1} \beta$-carotene and other carotenoids but with no adverse effect on plant morphology and development
Naqui et al. $2009 b$

Zhu et al. 2008 


\section{$c r t B$ and $c r t I$}

Erwinia herbicola

Super $\gamma$-zein

\section{Rice}

psy

crtI

psy

crtI

psy

\author{
Narcissus \\ pseudonarcissus
}

ETwinia uredovora

N. pseudonarcissus

E. uredovora

N. pseudonarcissus

\section{Endosperm}

specific rice glutelin

CaMv 35S

Endospermspecific glutelin

\section{CaMV 35S}

Endosperm-

specific rice glutelin
Overexpression of $c r t B$ and $c r t I$ resulted in an increase of total carotenoids of up to 34-fold with a preferential accumulation of $\beta$-carotene in maize endosperm, which remained reproducible over at least four generations

Polished seeds of transgenic rice showed the presence of higher accumulation of $\beta$-carotene, $\mathrm{Fe}$ and $\mathrm{Zn}$ compared to WT; prussian blue staining revealed the presence of $\mathrm{Fe}$ in the endosperm cells of transgenic rice seeds compared to WT where Fe restricted only to aleurone and embryo

BR29 rice containing psy and crtI showed up to $9.34 \mathrm{\mu g} \mathrm{g}^{-1}$ total carotenoids, with $\beta$-carotene approaching $3.92 \mathrm{\mu g} \mathrm{g}^{-1}$ in polished seeds; the total carotenoids and $\beta$-carotene in polished seeds of transgenic rice IR64, respectively, 2.32 and $0.96 \mu g g^{-1}$

Golden Rice 2 containing $p s y$ and crtI showed a total carotenoids of up to 23 -fold (maximum $37 \mu \mathrm{gg}^{-1}$ ), with a preferential accumulation of $\beta$-carotene (31 $\mathrm{gg} \mathrm{g}^{-1}$ ), compared to the original Golden Rice $\left(1.6 \mu \mathrm{gg}^{-1}\right.$ ) (Ye et al. 2000); transgene had no adverse effect on plant phenotype, seed weight, or germination
Aluru et al. 2008

Sellappan et al. 2009

Datta et al. 2006

\section{Paine et al.} 2005

(continued) 


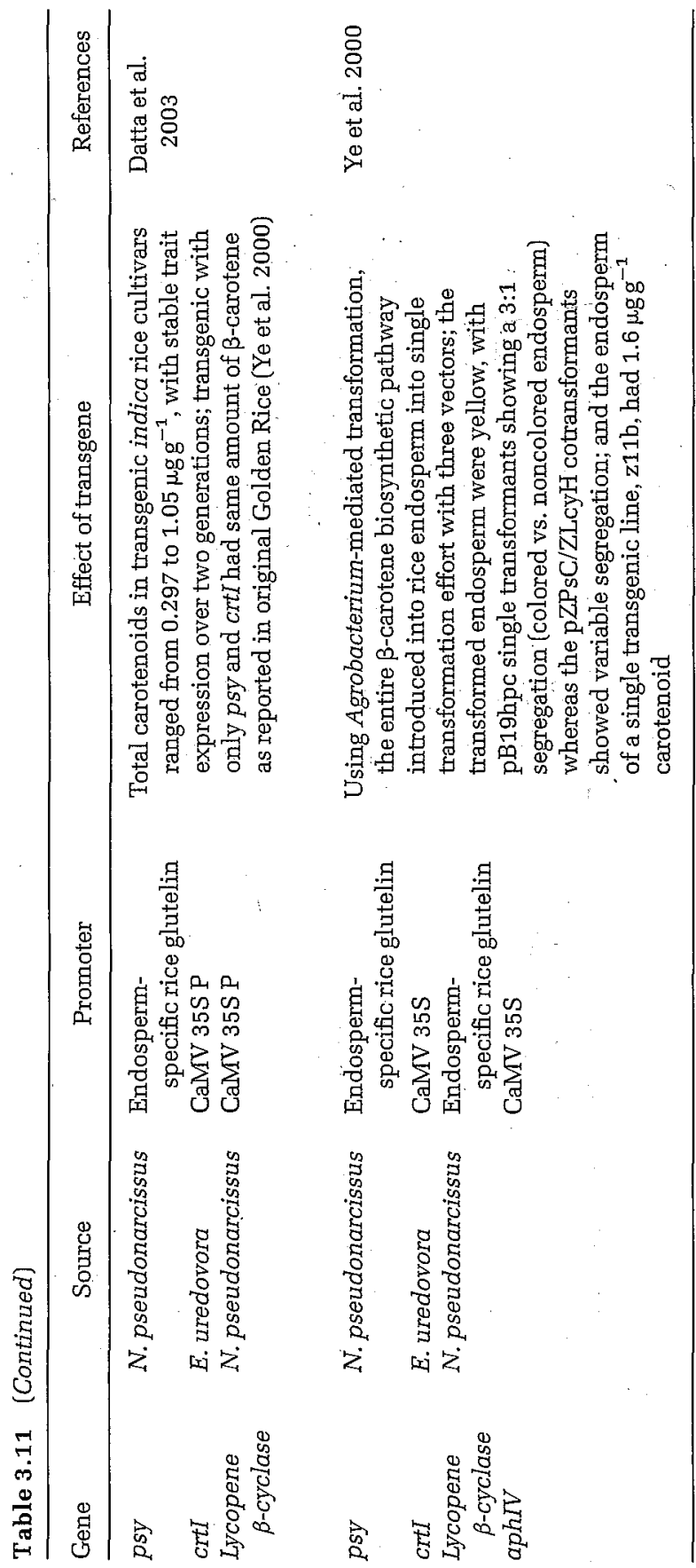


et al. 2008), which remained stable over four generations and that the increased accumulation of $\beta$-carotene is due to an up-regulation of the endogenous lycopene $\beta$-cyclase.

Recent developments in the genomic revolution provide researchers a great deal of information, which can be derived from studying many genes or proteins simultaneously using multigene transfer (MGT) as an approach to generate plants with more ambitious phenotypes, for example, the import of entire metabolic pathways, the expression of entire protein complexes, the development of transgenic crops simultaneously engineered to produce a spectrum of added-value compounds, with limitless potential (Naqvi et al. 2009a). This approach has been recently employed to generate transgenic maize containing four to five genes to enhance the nutritional profiles of the maize kernels (Table 3.11). For example, transgenic maize containing psy1, crtI, dhar, and folE genes under endosperm-specific promoters contains 169-fold the normal amount of $\beta$-carotene, sixfold the normal amount of ascorbate, and double the normal amount of folate (Naqvi et al. 2009b). Zhu et al. (2008) introduced five carotenoid genes, psy1, crtI, lycopene $\beta$-cyclase, bch, and crtW in a South African white maize cultivar 'M37W'. The transgenic events express different enzyme combinations to show distinct metabolic profiles, with some accumulating up to $57.35 \mu \mathrm{gg}^{-1} \beta$-carotene and other carotenoids but with no adverse effect on plant morphology and development, which has allowed them to identify and complement rate-limiting steps in the pathway and to demonstrate competition between $\beta$-carotene hydroxylase and bacterial $\beta$-carotene ketolase in four sequential steps of the extended pathway.

Thus, the combinatorial transformation is a versatile approach that could be used to modify any metabolic pathway and pathways controlling other biochemical, physiological, or developmental processes. These examples clearly demonstrate that through use of transgenic technology, it is possible to alter the seed composition to make food crops more nutritious to human health. However, use of transgene still has some practical limitations, for example, mechanism and pattern of gene integration, dosage effect due to variable copy insertion, interaction between transgenes, rearrangements and silencing, promoter choice as a function of gene number. (i.e., one promoter vs. more promoters when integrating multiple genes) as repetitive promoters may in some cases have negative impact on transgene stability and expression, or proper coordination of all enzymes involved in the metabolic pathway. Transfer of an incomplete pathway induces significant changes in plant morphology, variable expression of transgene effects in different generation and some lines with more expression than 
others in later generations etc (Kristensen et al. 2005; Naqvi et al. 2009a,b; Dietz-Pfeilstetter 2010; Peremarti et al. 2010).

It is, therefore, evident that the best approach to address human nutrition through biofortification strategies will likely involve genetic engineering in conjunction with conventional breeding, particularly when the direct enhancement of local elite breeding is required (Datta et al. 2007; Naqvi et al. 2009a,b; Ronald 2011).

\section{B. Consumer's Attitude to Genetically Modified Biofortified Crops}

Biofortification often alters the flavor, taste, appearance (not in the case of $\mathrm{Fe} / \mathrm{Zn}$ ), and other features of foods that may limit consumer acceptance of the nutritionally enhanced GM food. It is, therefore, worth investigating the social and economic impacts of nutritionally enhanced transgenic plants such as the cost-effectiveness of adapting local cultivars, social acceptance of the strategy, willingness to pay for genetically modified food (Kimenju and Groote 2008; Stevens and Winter-Nelson 2008; González et al. 2009), and the overarching regulatory policy (see Section VII.C) for producing such crops on an agronomic scale (Ramessar et al. 2008). The proof of concept of developing nutritionally enhanced biofortified crops through genetic engineering have been demonstrated in maize and rice (see Section VII.A). The overexpression of genes associated with increased Fe concentration did not change the seed color in rice, while enhanced $\beta$-carotene resulted golden-yellow seeds in rice, orange-yellow seeds in maize; and orange colored cassava and sweet potato. Skepticism prevails among public in large about the acceptance of, for example, $\beta$-carotene-enriched rice (golden rice), cassava, and sweet potato. The consumers in southern Africa have strong preference for white maize (which has no $\beta$-carotene) and often consider yellow maize being inferior, suitable only for animal feed (Rubey et al. 1997). How far the biofortified $\beta$-carotene-enriched maize will be accepted by the consumers who prefer white maize for consumption is a question remaining to be answered. A survey conducted in Kenya reveals that consumer preference is influenced by socioeconomic factors such as gender, education, and income or ethnic background. For example, in spite of the strong preference for white maize, many would prefer to eat yellow (biofortified) maize if offered a price discount $(\sim 37 \%)$ over white maize. Women being more sensitive to nutrition have shown preference to eat biofortified maize than men, and urban consumers (due to raise in income and more awareness about the micronutrient malnutrition) have shown a willingness to pay for biofortified maize. Consumers from western Kenya have a lower 
preference for white maize, while those from central Kenya a stronger preference for biofortified yellow maize (Groote and Kimenju 2008). The survey results also reveal that biofortified $\beta$-carotene-enriched maize and OFSP in Mozambique and cassava in northeast Brazil is acceptable to many consumers in these countries (Low et al. 2007; Stevens and Winter-Nelson 2008; González et al. 2009). The yellow gari (made from biofortified cassava containing high $\beta$-carotene) in Nigeria is fetching a higher price than white gari (HPlus Nigeria Project). Furthermore, in a recent study on acceptance of biofortified sweet potato in Uganda, Chowdhury et al. (2011) found that taste plays an important role in consumer acceptance; however, the provision of nutrition information does translate into substantial premiums for the biofortified sweet potato relative to the more common white cultivars. The consumers in Uganda are willing to pay for biofortified cultivars as much they are for the currently consumed traditional (white) cultivars.

The adoption of nutritionally enhanced food crops will improve the health and well-being of the world's poorest people, but this advancement will only be possible if political differences over the development and use of transgenic crops are set aside and their deployment and cultivation is regulated according to robust, science-based criteria (Naqvi ét al. 2009a,b; Ramessar et al. 2009; Gómez-Galera et al. 2010).

\section{Nutritionally, Enhanced Genetically Modified Crops and Biosafety Issues}

The GM crops are currently classified in generations and according to the objective of the trait being introduced. The first generation GM crops include those that possess resistance to herbicide and/or insect pests (input trait), while the second generation GM crops include those with new traits of direct value to consumers such as improved grain quality (output trait). The third generation of GM crops being developed is expected to confer plants a greater ability to resist abiotic stresses (drought, salinity and high temperatures), provide additional health benefit or renewable raw materials (bioenergy-rich crops) or pharmaplants to produce high-grade active pharmaceutical ingredients (Magaña-Gómez and de la Barca 2008). The first generation GM crops are the herbicide-resistant soybean, insect-resistant maize and cotton, herbicide- and insect-resistant potato; the second generation GM crops are rice with enhanced $\beta$-carotene and/or higher iron and zinc levels or maize with high phytase, while maize with enhanced multiple vitamins and minerals could be classified as third generation crop (see Section VII.A). The herbicide- and/or insect pest-resistant soybean, 
maize, and cotton are commercially grown in 125 million ha in 25 countries (James 2008), while nutritionally enhanced GM crops are yet to be commercialized. The future waits for the success or limitations of second and third generation transgenics.

The major concern about the GM crops/foods is the safety to humans and animals. The potential risks to human health include toxicity, allergenicity, the instability of inserted gene, and negative effects on nutrition (Conner and Jacobs 2000). During 1996-2010, a large number of studies on the safety assessment of GM crops (maize, peas, potato, rice, soybean, sweet pepper, and tomato) or GM traits (herbicide, insect, and virus resistance) have been conducted using various feeding periods, animal models (broiler, catfish, chicken, cow, dairy cattle, mouse, mouse testes, rabbit, rat, salmon, sheep, and steer), and parameters (body weight, feed consumption, blood chemistry, organ weight, and histopathology). The most common findings from these investigations have varied from no alteration of the nutritional value of the GM food tested to minimal detrimental effects on the nutritional value to in vivo submicroscopic effects in different animal species.

Differences among the methods employed for evaluation, and the varied results obtained on the risk assessments reflect the complexity of the problem and therefore, there is an urgent need to harmonize the methods used to evaluate the safety of the GM foods (Flachowsky et al. 2007; Magaña-Gómez and de la Barca 2008; Magaña-Gómez et al. 2008; de Vendômois et al. 2009; Haryu et al. 2009; Steinke et al. 2010). Rigorous, multigenerational animal safety assessments should be done to identify the risks to health, and all the GM products including those nutritionally enhanced by biofortification must be labeled to be monitored for the long-term adverse health consequences due to their consumption.

The detection and characterization of the unintended effects of the genetic modification, as demonstrated in maize (higher lignin content in Bt maize by Saxena and Stotzky 2001) and soybean (depleted plant flavonoids in herbicide-tolerant soybeans by Lappé et al. 1999), continues to be an issue that needs further investigation. The newly developed methods of screening for potential alterations in the metabolism of the modified organism include the analysis of gene expression (microarrays, mRNA finger printing), overall protein analysis (proteomics), and secondary metabolites (HT-UHPLS-TOF-MS, MRM-TQMS; Grata et al. 2009; Sawada et al. 2009; Allwood and Goodacre 2010) that should be integrated into the risk assessment process. To sum up, the advances made in molecular biology, toxicology, biochemistry, and 
nutrition hold the promise of providing sets of genes and methodologies that serve as biomarkers for a cell's responses to toxins, allergens, or other compounds, which hold potential to the development of new tools to assess the GM crops. Further, the next step for a safe use of the GM crops is to adopt strictly the recommendations made available by the regulatory agencies to ensure that the consumption of GM foods does not pose a serious health hazard to humans, animals, and biota (Magaña-Gómez and de la Barca 2008).

It is encouraging to note that a number of countries have put in place the laws and biosafety regulations governing the tolerance levels for the GM materials in nonGM food and in the labeling and traceability of the GM products. However, in many developing countries, there appears to be no tolerance limit to distinguish the GM and nonGM food and feed. Furthermore, there is no uniformity in the approach to adopting the labeling and traceability of the GM food among the countries, that is, while some adopted voluntary leveling of the GM products, others adopted mandatory labeling of GM products, still others have no requirements for labeling at all, which is a serious problem when such GM crops/products are traded for food and feed uses, therefore an urgent need exists to harmonize the regulations on a global level (Ramessar et al. 2008).

The environmental risks associated with the GM crops such as gene flow, adverse effects on biodiversity and on the beneficial insects, and the potential emergence of superweeds are not referred to here as these issues have been adequately dealt elsewhere (Wolfenbarger and Phifer 2000; Andow and Zwahlen 2006; Kwaku and Asante 2008; Dunfield and Germida 2010; Hokanson et al. 2010; Jiang et al. 2010; Liu 2010; Raybould et al. 2010).

In summary, the ferritin gene either from soybean or common bean or NAS gene from Arabidopsis driven by endosperm-specific promoters have been used to enhance seed-Fe concentration. The transformed rice containing AtNAS and Pvferritin has increased iron several fold compared to WTs with no yield penalty. Phytate limits the Fe bioavailability. The transgenic rice containing RINO1 shows substantial reduction in phytic acid, but with corresponding increase in available inorganic phosphorus, and the produce from such plants when sown had no adverse effects on seed germination, plant growth, and development, unlike lp mutants. The transgenic maize containing phyA2 from $A$ spergillus shows high phytase activity and reduces phytic acid compared to WT. A new version of Golden Rice named as 'Golden Rice 2' has been developed, which has exceptionally high amount of $\beta$-carotene ( $31 \mu \mathrm{g} \mathrm{g}^{-1}$ seed), which is available to the public domain for large-scale evaluation 
and introgression into locally adapted rice cultivars. Multigene transfer is an approach to generate plants with more ambitious phenotypes. Transgenic maize containing five carotenoid genes show higher accumulation of $\beta$-carotene with no adverse effects on plant morphology and development. It has been clearly demonstrated that it is possible to alter the seed composition, using transgene(s), to make food crops more nutritious to human health; however, the use of transgene still has some practical limitations, highlighted in this section; which must be addressed to minimize failures and disappointments.

Biofortification has the potential to alter the flavor, taste, or appearance, which may limit consumer acceptance. The enhanced $\beta$-carotene results in golden-yellow colored seeds in rice, the orange-yellow seeds in maize, and orange in cassava and sweet potato. The limited survey of $\beta$-carotene-enriched maize or orange-fleshed sweet potato and cassava in some countries in Africa and northeast of Brazil reveals that such products are acceptable to many consumers. More such studies are needed to gauge consumer's preference to biofortified crops. The deployment and cultivation of nutritionally enhanced GM crops will succeed only if the political differences over the development and use of GM crops are set aside and a rigorous assessment is in place based on robust, science-based criteria to assess biosafety issues associated with the use of GM crops/products.

\section{OUTLOOK}

Limited studies involving germplasm have shown potential variability that merits further exploration to mine genetic variation for grain Fe, Zn, phytate, and $\beta$-carotene in.germplasm collections. The core or mini-core collections available in most of the cereal and legume crops should form the basis to identify seed mineral-dense germplasm. Priority may be given to screen germplasm from regions with soils deficient in micronutrients as the germplasm from such regions generally develops inherent adaptation mechanisms, favoring enhanced nutrient uptake, transport, distribution, and relocation in plants/seeds. Wild relatives are another valuable resource to explore variation for micronutrients, as evidenced in common bean and wheat. Although few major QTL associated with increased seed$\mathrm{Fe} / \mathrm{Zn}$ concentrations and/or $\mathrm{Fe}$ bioabailability have been reported in common bean, maize, rice, and wheat; more such studies are needed to understand the genetics of seed micronutrients concentration and bioavailability. The response of cultivars to the production 
environments need to be characterized to further improve the probability of predicting and identifying seed mineral-dense germplasm/cultivars. A useful strategy would be to characterize the production environment prior to evaluating germplasm and breeding lines in the target environments for identifying seed mineral-dense lines adapted to such production environments. The bioavailability of micronutrients is an issue that should be factored in when selecting for seed mineral-dense cultivars; however, such studies are costly and time consuming. The in vitro Caco- 2 cell assay should be used as a first step to assess the bioavailability, while the in vivo assay may be limited only to potential cultivars/hybrids to complement the in vitro results, prior to their release for commercial cultivation.

Nutrient acquisition in plants is largely influenced by root architecture, root exudates, and mycorrhizal associations. How nutrients from the soils are absorbed, transported, and partitioned into various plant parts and localized in seeds merit further investigation to develop selection criteria for the development of seed mineral-dense cultivars. High nutrient-use efficiency, as measured by high harvest index for micronutrients $\left(\mathrm{HI}_{\mathrm{Fe}}\right.$ or $\left.\mathrm{HI}_{\mathrm{Zn}}\right)$ should be integrated in breeding programs together with high $\mathrm{HI}_{\text {seed yield. }}$

High-throughput assays are needed to facilitate large-scale screening of seed samples for micronutrient concentration. XRF has emerged as a rapid and cost-effective assay for initial screening to discard lines in the lower range of $\mathrm{Fe}$ and $\mathrm{Zn}$. Subsequently, promising lines must be analyzed for variation by ICP. or AAS methods. The accumulation and spatial distribution of macro- and micronutrients provide elemental maps in whole seed, and the information from such distribution pattern should be integrated into the selection strategies for the biofortification of staple crops. Such information is also warranted to minimize micronutrient losses during the milling/polishing processes.

The proof of concept has already been demonstrated that it is possible to develop seed micronutrient-dense biofortified crops by exploiting natural genetic variation with no yield penalty, as evidenced in maize, cassava, and sweet potato ( $\beta$-carotene), common bean, pearl millet, and rice (iron). Seed iron-dense common bean and rice in several countries in Latin America; seed iron-dense common bean in some countries in Africa; and seed iron-dense rice in the Philippines in Asia have been released for cultivation. The $\beta$-carotene biofortified máize in Kenya, Mozambique, Nigeria, and South Africa, and $\beta$-carotene biofortified sweet potato in Mozambique and Uganda and cassava in Nigeria and DR Congo, all derived by using naturally occurring allelic variation, are being assessed for adaptation, acceptability, and efficacy prior to release 
in these countries. A paradigm shift is needed to include biofortification in core breeding programs to assure that no crop cultivars that do not meet the minimum seed-micronutrient density are released for cultivation.

The use of transgenic approaches to enhance the nutritional quality of food crops have been demonstrated in rice ( $\beta$-carotene and iron) and maize ( $\beta$-carotene). Genetic variants of 'Golden Rice 2' are being introgressed to transfer high $\beta$-carotene trait into several Asian rice cultivars, which will soon be available in public domain (Barry 2011). Reducing phytic acid enhances the bioavailability of Fe/Zn, which could be achieved either by blocking the phytate biosynthetic pathway or degrading phytate in the developing seeds. Transgenic maize containing phyA2 or transgenic rice containing RINO1 showed substantial reduction in phytic acid, with no adverse effects on seed germination, plant growth, and development, unlike in lp mutants. Further, MGT through transgenic approach has shown several fold improvement in the nutritional profiles of maize kernels, suggesting that MGT is a viable option to import entire metabolic pathways with limitless potential to alter the seed composition and make food crops more nutritious for human health. However, it is important to address the environmental and biosafety issues associated with the use of transgenic crops.

The critical issue to investigate is (1) whether heterosis can be exploited to enhance seed $\mathrm{Fe}$ and $\mathrm{Zn}$; (2) whether simultaneous selection can be practiced to enhance per se the micronutrients ( $\mathrm{Fe}$ and $\mathrm{Zn}$ ) and their bioavailability; (3). how much low phytate per se can be reduced that is not detrimental to plants and the produce is beneficial to human health; (4) whether low-phytate trait can be combined with increased grain $\mathrm{Fe}$ and $\mathrm{Zn}$ concentrations; and (5) whether $\mathrm{Fe} / \mathrm{Zn}$ (invisible trait) can be combined with marker (such as grain color) to differentiate between biofortified and nonbiofortified produce in the market. The equally important issues with respect to provitamin A research include (1): whether heterosis can be exploited to enhance $\beta$-carotene, (2) whether allelic variation for additional genes in carotenoids biosynthetic pathway be identified to reduce postharvest/storage losses, (3) whether enhancers of $\beta$-carotene bioavailability be identified and selected in breeding, (4) whether a rapid and costeffective high-throughput assay be developed to enhance breeding efficiency, and (5) whether carotenoids have mycotoxin-reducing effects in grain (Pixley et al, 2011b). More studies are needed to assess the efficacy of biofortified products in raising micronutrient status in human subjects. Biofortification often alters the flavor, taste; appearance, and other features of the foods, which may limit the consumer acceptance-an issue that merits further investigation. To address these 
and other issues, a network of global interdisciplinary partnership is suggested with a range of stakeholders, including those involved in breeding, molecular biology, food technology, human nutrition, economics, seed systems, farm extension, food product development, marketing, and public awareness to strengthen crop biofortification programs both in the developed and developing countries (Bouis and Islam 2011). The biofortified crops must be adopted by farmers and consumed by those suffering from micronutrient malnutrition. To make this happen, it is suggested that the biofortified produce should be made available to public through the public food distribution system (PFDS), which can create an institutional demand for biofortification and will surely work if high volumes of biofortified crops are procured through the PFDS, targeting the poor. The PFDS has the potential to induce farmers to cultivate biofortified crops by providing price support for production. Moreover, for enhancing the integration of biofortification into the PFDS, it is important that policymakers are made aware of the benefits of biofortification (Ahmed 2011).

\section{ACKNOWLEDGMENTS}

Appreciation is expressed to the staff of ICRISAT library for their efforts in conducting literature searches and arranging for reprints; to Kevin Pixley, the University of Wisconsin, Madison, USA and CIMMYT, Mexico for critically going through the provitamin A section of the manuscript; and to Jules Janick and the anonymous reviewers for making useful suggestions on improving the manuscript. Funding support from HarvestPlus is acknowledged as is the support and encouragement from William Dar (Director General, ICRISAT).

\section{LITERATURE CITED}

Abbo, S., C. Molina, R. Jungmann, M.A. Grusak, Z. Berkovitch, R. Reifen, G. Kahl, P. Winter, and R. Reifen. 2005. Quantitative trait loci governing carotenoid concentration and weight in seeds of chickpea (Cicer arietinum L.). Theor. Appl. Genet. 111:185-195.

Abdalla, A.A., A.H. El Tiany, B.E. Mohamed, and A.H. Abdalla. 1998. Proximate composition, starch, phytate and mineral contents of 10 pearl millet genotypes. Food Chem. 63:243-246.

Abilgos-Ramos, R.G., R.V. Manaois, S.S.P. Escubio, G.D.G. Garcia, E.C. Arocena, and L.S. Sebastian. 2004. Grain quality and iron density of Philippine rice cultivars. 4th Int. Crop Sci. Congress, Brisbane, Australia (http://www.cropscience.org.au).

Acosta-Gallegos, J.A., J.D. Kelly, and P. Gepts. 2007. Prebreeding in common bean and use of genetic diversity from wild germplasm. Crop Sci. 47 (S3): S44-S59. 
Ahmed, A.U. 2011. Getting biofortification into the public food distribution system. Symposia brief: Delivering iron/zinc crops: An invisible nutrient. First Global Conference on Biofortification. November 9-11, Washington, DC.

Akalu, G., S. Taffesse, N.S. Gunratna, and H. de Groote. 2010. The effectiveness of quality protein maize in improving the nutritional status of young children in the Ethiopian highlands. Food Nutr. Bull. 31:418-430.

Akond, A.S.M.G.M., H. Crawford, J. Berthold, Z.I. Talukder; and K. Hossain. 2011. Minerals ( $\mathrm{Zn}, \mathrm{Fe}, \mathrm{Ca}$ and $\mathrm{Mg}$ ) and antinutrient (Phytic acid) constituents in common bean. Am. J. Food Technol. 6:235-243.

Ali, M.Y., L. Krishnamurthy, N.P. Saxena, O.P. Rupela, J. Kumar, and C. Johansen. 2002. Scope for genetic manipulation of mineral acquisition in chickpea. Plant Soil 245:123-134.

Ali, M. and S.C.S. Tsou, 1997. Combating micronutrient deficiencies through vegetables: A neglected food frontier in Asia. Food Policy 22:17-38.

Alloway, B.J. 2009. Soil factors associated with zinc deficiency in crops and humans. Environ. Geochem. Health 31:537-548.

Allwood, J.W. and R. Goodacre. 2010. An introduction to liquid chromatography-mass spectrometry instrumentation applied in plant metabolomic analyses, Phytochem. Anal. 21:33-47.

Aluru, M., Y. Xu, R. Guo, Z. Wang, S. Li, W. White, K. Wang, and S. Rodermel. 2008. Generation of transgenic maize with enhanced provitamin A content. J. Exp. Bot. 59:3551-3562.

Alvarez-Hernandez, X., G.M. Nichols, and J. Glass. 1991. Caco-2 cell line: A system for studying intestinal iron transport across epithelial cell monolayers. Biochim. Biophys. Acta 1070:205-208.

Anandan, A., G. Rajiv, R. Eswaran, and M. Prakash. 2011. Genotypic variation and relationships between quality traits and trace elements in traditional and improved rice (Oryza sativa L.) genotypes. J. Food Sci. 76:H122-H130.

Andaya, C.B. and T.H. Tai. 2005. Fine mapping of the rice low phytic acid (Lpa1) locus. Theor. Appl. Genet. 111:489-495.

Andow, D.A. and C. Zwahlen. 2006. Assessing environmental risks of transgenic plants. Ecol. Lett. 9:196-214.

Andree, K.B., J. Kim, C.P. Kirschke, J.P. Gregg, H.Y. Paik, H. Joung et al. 2004. Investigation of lymphocyte gene expression for use as biomarkers for zinc status in humans. J. Nutr. 134:1716-1723.

Ariza-Nieto, M., M.W. Blair, R.M. Welch, and R.P. Glahn. 2007. Screening of iron. bioavailability patterns in eight bean (Phaseolus vulgaris Li) genotypes using the Caco-2 cell in vitro model. J. Agric. Food Chem. 55:7950-7956.

Arnason, J.T., J.D.H. Lambet, J. Gale, J. Mihm, M. Bajarnason, D. Jewell, J.A. Serratos, J. Fregeau-Reid, and L. Pietrzak. 1993. Is quality protein maize more susceptible than normal cultivars to attack by the maize weevil Sitophilus zeamais? Postharvest Biol. Technol. 2:349-358.

Arsenault, J.E., E.A. Yakes, M.B. Hossain, M.M. Islam, T. Ahmed, C. Hotz, B. Lewis, A.S. Rahman, K.M. Jamil, and K.H. Brown. 2010. The current high prevalence of dietary zinc inadequacy among children and women in rural Bangladesh could be substantially ameliorated by zinc biofortification of rice. J. Nutr. 140:1683-1690.

Astudillo, C. and M.W. Blair. 2008. Evaluación del contenido de hierro y zinc en semilla y su respuesta al nivel de fósforo en variedades de fríjol colombianas. Agronomia Colombiana 26:471-476. 
Atlin, G.N., N. Palacious, R. Babu, B. Das, S. Twumasi-Afriyie, D.K. Friesen, H. De Groote, B. Vivek, and K.V. Pixley. 2011. Quality protein maize: Progress and prospects. Plant Breed. Rev. 34:83-130.

$\mathrm{Au}$, A.P. and M.B. Reddy. 2000. Caco-2 cells can be used to assess human iron bioavailability from a semipurified meal. J. Nutr. 130:1329-1334.

Ayoya, M.A., G.M. Spiekermann-Brouwer, R.J. Stoltzfus, E. Nemeth, J.-P. Habicht, T. Ganz, R. Rawat, A.K. Traoré, and C. Garza. 2010. $\alpha_{1}$-Acid glycoprotein, hepcidin, C-reactive protein, and serum ferritin are correlated in anemic school children with Schistosoma haematobium. Am. J. Clin. Nutr. 91:1784-1790.

Badone, F.C., E. Cassani, M. Landoni, E. Doria, D. Panzeri, C. Lago, F. Mesiti, E. Nielsen, and R. Pilu. 2010. The low phytic acid-241 (Ipa1-241) maize mutation alters the accumulation of anthocyanin pigment in the kernel. Planta 231:1189-1199.

Baisakh, N., S. Rehana, M. Rai, N. Oliva, J. Tan, D.J. Mackill, G.S. Khush, K. Datta, and S.K. Datta. 2006. Marker-free transgenic (MFT) near-isogenic introgression lines (NILs) of golden indica rice (cv. IR64) with accumulation of provitamin A in the endosperm tissue. Plant Biotechnol. J. 4:467-475.

Baker, D.H. 2008. Animal models in nutrition research. J. Nutr. 138:391-396.

Baligar, V.C., N.K. Fageria, and Z.L. He. 2001. Nutrient use efficiency in plants. Commun. Soil Sci. Plant Anal. 32:921-950.

Ballot, D., R.D. Baynes, T.H. Bothwell, M. Gillooly, B.J. Macfarlane, A.P. Macphail, G. Lyons, D.P. Derman, W.R. Bezwoda, J.D. Torrance, and J.E. Bothwell. 1987. The effects of fruit juices and fruits on the absorption of iron from a rice meal. Br. J. Nutr. $57: 331-343$.

Bänziger, M. and J. Long. 2000. The potential for increasing the iron and zinc density of maize through plant-breeding. Food Nutr. Bull. 21:397-400.

Barrett, C.B. 2010. Measuring food insecurity. Science 327:825-828.

Barry, G. 2006. High-iron and zinc rice. In: Accounting Principles Fifth Canadian Edition Vol 1 Rice fact sheet. The International Rice Research Institute, Manila, The Philippines. Barry, G, 2011. Navigating the regulatory system: Lessions learned from golden rice. Symposia Brief: Delivering Vitamin A crops: A visible nutrient. First Global Conference on Biofortification. November 9-11, 2010, Washington, DC.

Baruah, A.M. and S. Borah. 2009. An investigation on sources of potential minerals found in traditional vegetables of Northeast India. Int. J. Food Sci. Nutr. 60 (Suppl. 4): 111-115.

Beard, J., R.-A. deRegnier, M.D. Shaw, R. Rao, and M. Georgieff. 2007. Diagnosis of iron deficiency in infants. Labmedicine 38:103-108.

Beard, J.L., L.E. Murray-Kolb, F.J. Rosales, N.W. Solomons, and M.L. Angelilli. 2006. Interpretation of serum ferritin concentrations as indicators of total-body iron stores in survey populations: The role of biomarkers for the acute phase response. Am. J. Clin. Nutr. 84:1498-1505.

Bechoff, A., D. Dufour, C. Dhuique-Mayer, C. Maruźe, M. Reynes, and A. Westby, 2009. Effect of hot air, solar and sun drying treatments on provitamin A retention on orangefleshed sweet potato. J. Food Eng. 92:164-171.

Bechoff, A., A. Westby, C. Owori, G. Menya, C. Dhuique-Mayer; D: Dufour, and K. Tomlins. 2010. Effect of drying and storage on the degradation of total carotenoids in orangefleshed sweet. potato cultivars. J. Sci. Food Agric. 90:622-629.

Bengtsson, A., A. Namutebi, M.L. Alminger, and U. Svanberg. 2008. Effect of various traditional processing methods on the all-trans- $\beta$-carotene content of orange-fleshed sweet potato. J. Food Comp. Anal. 21:134-143. 
Berger, J.D. 2007. Ecogeography and evolutionary approaches to improving adaptation of autumn-sown chickpea (Gicer arietinum L.) to terminal drought: The search for reproductive chilling tolerance. Field Grops Res. 104:112-122.

Berger, J.D., S.P. Milroy, N.C. Turner, K.H.M. Siddique, M. Imtiaz, and R. Malhotra. 2011. Chickpea evolution has selected for contrasting phenological mechanisms among different habitats. Euphytica 1801-15.

Bhaskarachary, K., D.S. Sankara Rao, Y.G. Deosthale, and V. Reddy. 1995. Carotene content of some common and less familiar foods of plant origin. Food Chem. 54:189193.

Bhullar, N.K., K. Street, M. Mackay, N. Yahiaoui, and B. Keller. 2009. Unlocking wheat genetic resources for the molecular identification of previously undescribed functional alleles at the Pm3 resistance locus. Proc. Natl. Acad. Sci. USA 106:9519-9524.

Bilyeu, K.D., P. Zeng, P. Coello, Z.J. Zhang, H.B. Krishnan, A. Bailey, P.R. Beuselinck, and J. C. Polacco; 2008, Quantitative conversion of phytate to inorganic phosphorus in soybean seeds expressing a bacterial phytase. Plant Physiol. 146:468-477.

Black, R.E., L.A. Allen, Z.A. Bhutta, L.E. Caulfield, M. de Onis, M. Ezzati, C. Mathers, and J. Rivera. 2008. Maternal and child undernutrition: Global and regional exposures and health consequences. Lancet 371:243-260.

Blair, M.W., C.A. Astudillo, S.E. Beebe, I. Roa, P. Kimani, and R. Chirwa. 2009a. Biofortification of common bean (Phaseolus vulgaris L.) via traditional and novel breeding approaches. J. Danish Biochem. Soc. (Biozoom) 1:25-28.

Blair, M.W., C. Astudillo, M.A. Grusak, R. Graham, and S.E. Beebe. 2009b. Inheritance of seed iron and zinc concentrations in common bean (Phaseolus vulgaris L.). Mol. Breed. 23:197-207.

Blair, M.W., C. Astudillo, J. Rengifo, S.E. Beebe, and R. Graham. 2011. QTL for seed iron and zinc concentrations in a recombinant inbred line population of Andean common beans (Phaseolus vulgaris L.). Theor. Appl. Genet. 122:511-523.

Blair, M.W., L.F. Gonzales, P. Kimani, and L. Butare. 2010a. Inter-genepool introgression, genetic diversity and nutritional quality of common bean (Phaseolus vulgaris L.) landraces from Central Africa. Theor. Appl. Genet. 121:237-248.

Blair, M.W., S.J.B. Knewtson, C. Astudillo, C.-M. Li, A.C. Fernandez, and M.A. Grusak. $2010 \mathrm{~b}$. Variation and inheritance of iron reductase activity in the roots of common bean (Phaseolus vulgaris L.) and association with seed iron accumulation QTL. BMC Plant Biol. 10:215.

Blair, M.W., J.I, Medina, C. Astudillo, J. Rengifo, S.E. Beebe, G. Machado, and R. Graham. 2010c. QTL for seed iron and zinc concentrations in a recombinant inbred line population of Mesoamerican common beans (Phaseolus valgaris L.). Theor. Appl. Genet. 121:1059-1071.

Blair, M.W., F. Monserrate, S.E. Beebe, J. Restrepo, and J.O. Flores. 2010d. Registration of high mineral common bean germplasm lines NUA35 and NUA56 from the red-mottled seed class. J. Plant Regist. 4:55-59.

Blair, M.W., T. Porch, K. Cichy, C.H. Galeano, P. Lariguet, C. Pankurst, and W. Broughton. 2008. Induced mutants in common bean (Phaseolus vulgaris), and their potential use in nutrition quality breeding and gene discovery. Israel J. Plant Sci. 55:191-200.

Blair, M.W., T.A. Sandoval, G.V. Caldas, S.E. Beebe, and M.I. Páez, 2009c. Quantitative trait locus analysis of seed phosphorus and seed phytate content in a recombinant inbred line population of common bean. Crop Sci. 49:237-246.

Blandino, A., M.E. Al-Aseeri, S.S. Pandiella, D. Cantero, and C. Webb. 2003. Cereal-based fermented foods and beverages. Food Res. Int. 36:527-543. 
Blanquet-Diot, S., M. Soufi, M. Rambeau, E. Rock, and M. Alric. 2009. Digestive stability of xanthophylls exceeds that of carotenes as studied in in vitro gastrointestinal system. J. Nutr. 139:876-883.

Borel, P., P. Grolier, M. Armand, A. Partier, H. Lafont, D. Lairon, and V. Azais-Braesco, 1996. Carotenoids in biological emulsions: Solubility, surface-to-core distribution, and release from lipid droplets. J. Lipid Res. 37:250-261.

Borg, S., H. Brinch-Pedersen, B. Tauris, and P.B. Holm. 2009. Iron transport, deposition and bioavailability in the wheat and barley grain. Plant Soil 325:15-24.

Bouis, H.E. 2003. Micronutrient fortification of plants through plant breeding: Can it improve nutrition in man at low cost? Proc. Nutr. Soc. 62:403-411.

Bouis, H.E. 2007. The potential of genetically modified food crops to improve human nutrition in developing countries. J. Develop. Studies 43:79-96.

Bouis, H, and Y. Islam. 2011. Biofortification: Leveraging agriculture to reduce hidden hunger. IFPRI 2020 Int. Conference. Brief 19. February 10-12, 2011. New Delhi, India:

Bouis, H.E. and R.M. Welch. 2010. Biofortification: A sustainable agricultural strategy for reducing micronutrient malnutrition in the global south. Crop Sci. 50:S20-S32.

Bowen, D.E., M.J. Guttieri, K. Peterson, V. Raboy, and E.J. Souza, 2006. Phosphorus fractions in developing seeds of four low phytate barley (Hordeum vulgare L.) genotypes. Crop Sci. 46:2468-2473.

Bregitzer, P., and V: Raboy. 2006. Effects of four independent low-phytate mutations on barley agronomic performance. Crop Sci. 46:1318-1322.

Briat J.-F, and S. Lobreaux. 1997. Iron transport and storage in plants. Trends Plant Sci. 2:187-193.

Brinch-Pedersen, H., S. Borg, B. Tauris, and P.B. Holm. 2007. Molecular.genetic approaches to increasing mineral availability and vitamin content of cereals. J. Cereal Sci. 46:308-326.

Brown; A.H.D. 1989. Core collections: A practical approach to genetic resources management. Genome 31:818-824.

Brown, K.H., K.R. Wessells, and S.Y. Hess. 2007. Zinc bioavailability from zinc-fortified foods, Int. J. Vitam. Nutr. Res. 77:174-181.

Brunson; A.M., and F.W. Quackenbush. 1962. Breeding corn with high provitamin A in the grain. Crop Sci. 2:344-347.

Burt, A.J, C.M. Grainger, J.C. Young, B.J. Shelp, and E.A. Lee. 2010. Impact of postharvest handling on carotenoids concentration and composition in high-carotenoid maize (Zea mays L.) kemels. J. Agric. Food Chem. 58:8286-8292.

Cabuslay, G.S., C.B. Sison, E. Laureles, R. Buresh, W. Lazaro, and G.B. Gregorio. 2003. Grain mineral density: Nitrogen response and seasonal variation. Workshop on Breeding for Better Nutrition, 7-11 April 2003.

Cakmak, I. 2008. Enrichment of cereal grains with zinc: Agronomic or genetic biofortification? Plant Soil 302:1-17.

Cakmak, I., R. Derici, B. Torun, I. Tolay, H.J. Braun, and R. Schlegel. 1997a. Role of rye chromosomes in improvement of zinc efficiency in wheat and triticale. Plant Soil 196:249-253.

Cakmak, I., H. Ekiz, A. Yilmaz, B. Torun, N. Köleli, I. Gültekin, A. Alkan, and S. Eker. 1997b. Differential response of rye, triticale, bread and durum wheats to Zn deficiency in calcareous soils. Plant Soil 188:1-10.

Cakmak, I., H. Ozkan, H.J. Braun, R.M. Welch, and V. Romheld. 2000. Zinc and iron concentrations in seeds of wild, primitive, and modern wheats. Food Nutr. Bull. 21:401-403. 
Cakmak, I., W.H. Pfeiffer, and B. McClafferty. 2010. Review: Biofortification of durum wheat with zinc and iron. Cereal Chem. 87:10-20.

Cakmak, I., A. Torun, E. Millet, M. Feldman, T. Fahima, A. Korol, E. Nevo, H.J. Braun, and H. Özkan. 2004. Triticum dicoccoides: An important genetic resource for increasing zinc and iron concentrations in modern cultivated wheat. Soil Sci. Plant Nutr. 50:1047-1054.

Cakmak, I., A. Yilmaz, M. Kalayci, H. Ekiz, B. Torun, B. Erenoglu, and H.J. Braun. 1996. Zinc deficiency as a critical problem in wheat production in Central Anatolia. Plant Soil 180:165-172.

Calderini, D.F., and I. Ortiz-Monasterio. 2003. Are synthetic hexaploids a means of increasing grain element concentrations in wheat? Euphytica 134:169-178.

Campion, B., F. Sparvoli, E. Doria, G. Tagliabue, I. Galasso, M. Fileppi, R. Bollini, and E. Nielsen. 2009. Isolation and characterization of an Ipa (low phytic acid) mutant in common bean (Phaseolus vulgaris L.). Theor. Appl. Genet. 118:1211-1221.

Campos-Bowers, M.H., and B.F. Wittenmyer. 2007. Biofortification in Ghina: Policy and practice. Health Res. Policy Syst. 5:10.

Cao, J. and R.J. Cousins. 2000. Metallothionein mRNA in monocytes and peripheral blood mononuclear cells and in cells from dried blood spots increases after zinc supplementation of men. J. Nutr. 130:2180-2187.

Casa, A.M., G. Pressoir, P.J. Brown, S.E. Mitchell, W.L. Rooney, M.R. Tuinstra, C.D. Franks, and S. Kresovich. 2008. Community resources and strategies for association mapping in sorghum. Crop Sci. 48:30-40.

Casey, G.J., T.Q. Phuc, L. MacGregor, A. Montresor, S. Mihrshahi, T.D. Thach, N.T. Tien, and B.-A. Biggs. 2010. A free weekly iron-folic acid supplementation and regular deworming program is associated with improved hemoglobin and iron status indicators in Vietnamese women. BMC Public Health 9:261.

Castenmiller, J.J.M., and C.E. West. 1998. Bioavailability and bioconversion of carotenoids. Annu. Rev. Nutr. 18:19-38.

Cavagnaro, T.R. 2008. The role of arbuscular mycorrhizas in improving plant zinc nutrition under low soil zinc concentrations: A review. Plant Soil 304:315-325.

Chandel, G., S. Banerjee, and S.B. Verulkar. 2010. Expression profiling of metal homeostasis related candidate genes in rice (Oryza spp.) using semi quantitative RT-PCR analysis. Rice Genet. Newsl, 25:44-47.

Chander, S., Y.Q. Guo, X.H. Yang, J. Zhang, X.Q. Lu, J.B. Yan, T.M. Song, T.R. Rocheford, and J.S. Li. 2008. Using molecular markers to identify two major loci controlling carotenoid contents in maize grain. Theor. Appl. Genet. 116:223-233.

Chatzav, M., Z. Peleg, L. Ozturk, A. Yazici, T. Fahima, I. Cakmak, and Y. Saranga. 2010. Genetic diversity for grain nutrients in wild emmer wheat: Potential for wheat improvement. Ann. Bot. 125:529-531.

Chen, R., G. Xue, P. Chen, B. Yao, W. Yang, Q. Ma, Y. Fan, Z. Zhao, M.C. Tarczynski, and J. Shi. 2008. Transgenic maize plants expressing a fungal phytase gene. Transgenic Res. 17:633-643.

Cheng, L., F. Wang, H. Shou, F. Huang, L. Zheng, F. He, J. Li, F.-J. Zhao, D. Ueno, J.F. $\mathrm{Ma}$, and P. Wu. 2007. Mutation in nicotianamine aminotransferase stimulated the $\mathrm{Fe}$ (II) acquisition system and led to iron accumulation in rice. Plant Physiol. 145:16471657.

Chiera, J.M., J.J. Finer, and E.A. Grabau. 2004. Ectopic expression of a soybean phytase in developing seeds of Glycine max to improve phosphorus availability. Plant Mol. Biol. 56:895-904. 
Chhuneja, P., H.S. Dhaliwal, N.S. Bains, and K. Singh. 2006. Aegilops kotschyi and A. tauschii as sources for higher levels of grain iron and zinc. Plant Breed, 125:529-531.

Chowdhury, S., J.V. Meenakshi, K.I. Tomlins, and C. Owori. 2011. Are consumers in developing countries willing to pay more for micronutrient-dense biofortified foods? Evidence from a field experiment in Uganda. Am. J. Agric. Econ. 93:83-97.

Cichy, K.A., G.V. Caldas, S.S. Snap, and M.W. Blair. 2009. QTL analysis of seed iron, zinc, and phosphorus levels in an Andean bean population. Crop Sci. 49:1742-1750.

Collard, B.C.Y., and D.J. Mackill. 2008. Marker-assisted selection: An approach for precision plant breeding in the twenty-first century. Philos. Trans. R. Soc. Lond. Biol. Sci. 363:557-572.

Conner, A.J., and J.M. Jacobs. 2000. Food risks from transgenic crops in perspective. Nutrition 16:709-711.

Cvitanich, C., W.J. Przybyłowicz, J.M. Przybyłowicz, M.W. Blair, E. Jensen, and J. Stougaard. 2011. Micro-PIXE investigation of bean seeds to assist micronutrient biofortification. Methods Phys. Res. 269:2297-2302.

Cvitanich, C., W.J. Przybylowicz, D.F. Urbanski, A.M. Jurkiewicz, J. Mesjasz-Przybylowicz, M.W. Blair, C. Astudillo, E.O. Jensen, and J. Stougaard. 2010. Iron and ferritin accumulate in separate cellular locations in Phaseolus seeds. BMC Plant Biol. 10:26.

DaMatta, F.M., A. Grandis, B.C. Arenquae, and M.S. Buckeridge. 2010. Impact of climate changes on crop physiology and food quality. Food Res. Int. 43:1814-1823.

Darnton-Hill, I., P. Webb, P.W. Harvey, J.M. Hunt, N. Dalmiya, M. Chopra, M.J. Ball, M.W. Bloem, and B. de Benoist. 2005. Micronutrient deficiencies and gender: Social and economic costs. Am. J. Clin. Nutr. 81:1198S-1205S.

Datta, K., N. Baisakh, N. Oliva, L. Torrizo, E. Abrigo, J. Tan, M. Rai, S. Rehana, S. Al-Babili, P. Beyer, I. Potrykus, and S.K. Datta. 2003. Bioengineered "golden" indica rice cultivars with $\beta$-carotene metabolism in the endosperm with hygromycin and mannose selection systems. Plant Biotechnol. J. 1:81-90.

Datta, S.K., K. Datta, V. Parkhi, M. Rai, N. Baisakh, G. Sahoo, S. Rehana, A. Bandyopadhyay, Md. Alamgir, Md. S. Ali, E. Abrigo, N. Oliva, and L. Torrizo. 2007. Golden rice: Introgression, breeding, and field evaluation. Euphytica 154:271-278.

Datta, K., M. Rai, V. Parkhi, N. Oliva, J. Tan, and S.K. Datta. 2006. Improved "golden" indica rice and post-transgeneration enhancement of metabolic target products of carotenoids ( $\beta$-carotene) in transgenic elite cultivars (IR64 and BR29). Curr. Sci. 91:935-939.

Davis, D.R. 2005. Trade-offs in agriculture and nutrition. Food Technol. 59:120.

Davis, D.R. 2009. Declining fruit and vegetable nutrient composition: What is the evidence?. HortScience 44:15-19.

Davis, C.R., J.A. Howe, T.R. Rocheford, and S.A. Tanumihardjo. 2008. The xanthophylls composition of biofortified maize (Zea mays sp.) does not influence the bioefficacy of provitamin A carotenoids in Mongolian gerbils (Meriones unguiculatus). J. Agric. Food Chem. 56:6745-6750.

Déat, E., S. Blanquet-Diot, J.F. Jarrige, S. Denis, E. Beyssac, and M. Alric. 2009. Combining the dynamic TNO-gastrointestinal tract system with a Caco-2 cell culture model: Application to the assessment of lycopene and $\alpha$-tocopherol bioayailability from a whole food. J. Agric. Food Chem. 57:11314-11320.

Demirkiran, A.R. 2009. Determination of $\mathrm{Fe}, \mathrm{Cu}$ and $\mathrm{Zn}$ contents of wheat and corn grains from different growing site. J. Anim. Vet. Adv, 8:1563-1567.

de Rosa, S.S., N.D. Ribeiro, E. Jost, L.R.S. Reiniger, D.P. Rosa, T. Cerutti, and M.T.D.L.F, Póssobom. 2010. Potential for increasing the zinc content in common bean using genetic improvement. Euphytica 175:207-213. 
de Vendômois, J.S., F. Roullier, D. Cellier, and G.-E. Séralini. 2009. A comparison of the effects of three GM corn varieties on mammalian health. Int. J. Biol. Sci. 5:706-726.

Dietz-Pfeilstetter, A. 2010. Stability of transgene expression as a challenge for genetic engineering. Plant Sci. 179:164-167.

Distelfeld, A., I. Cakmak, Z. Peleg, L. Ozturk, A.M. Yazici, H. Budak, Y. Saranga, and T. Fahima. 2007. Multiple QTL effects of wheat $G p c-B 1$ locus on grain protein and micronutrient concentrations. Physiol. Plant. 129:635-643.

Douchkov, D., C. Gryczka, U.W. Stephan, R. Hell, and H. Baumlein. 2005. Ectopic expression of nicotianamine synthase genes results in improved iron accumulation and increased nickel tolerance in transgenic tobacco. Plant Cell Environ. 28:365-374.

Drakakaki, G., S. Marcel, R.P. Glahn, E.K. Lund, S. Pariagh, R. Fischer, P. Christou, and E. Stoger. 2005. Endosperm-specific co-expression of recombinant soybean ferritin and Aspergillus phytase in maize results in significant increases in the levels of bioavailable iron. Plant Mol. Biol. 59:869-880.

Dudal, R. 1976. Inventory of the major soils of the world with special reference to mineral stress hazards. p. 3-13. In: M.J. Wright (ed.), Accounting Principles Fifth Canadian Edition Vol 1 Plant adaptation to mineral stress in problem soils. Cornell University Press, Ithaca, New York, USA.

Duffus, C.M., and R. Rosie. 1976. Changes in trace element composition of developing barley grain. J. Agric. Sci. 87:75-79.

Dunfield, K.E., and J.J. Germida. 2010. Impact of genetically modified crops on soil- and plant-associated microbial communities. J. Environ. Qual. 33:806-815.

Dwivedi, S.L., M.W. Blair, H.D. Upadhyaya, R. Serraj, J. Balaji, H.K. Buhariwalla, R. Ortiz, and J.H. Grouch, 2005. Using genomics to exploit grain legume biodiversity in crop improvement. Plant Breed. Rev. 26:171-357.

Dwivedi, S.L., J.H. Grouch, D.J. Mackill, Y. Xu, M.W. Blair, M. Ragot, H.D. Upadhyaya, and R. Ortiz. 2007. The molecularization of public sector crop breeding: Progress, problems, and prospects. Adv. Agron. 95:163-318.

Egesel, C.O., J.C. Wong, R.J. Lambert, and T.R. Rocheford, 2003. Combining ability of maize inbreds for carotenoids and tocopherols. Crop Sci. 43:818-823.

El Bouhssini, M., K. Street, A. Joubi, Z. Ibrahim, and F. Rihawi. 2009. Sources of wheat resistance to Sunnzpest, Eurygaster integriceps Puton, in Syria. Genet. Resour. Crop Evol. 56:1065-1069.

Eneroth, H., S. el Arifeen, L.-A. Persson, B. Lönnerdal, M.B. Hossain, C.B. Stephensen, and E.-C. Ekström. 2010. Maternal multiple micronutrient supplementation has limited impact on micronutrient status of Bangladeshi infants compared with standard iron and folic acid supplementation. J. Nutr. 140:618-624.

Erbs, M., R. Manderscheid, G. Jansen, S. Sddig, A. Pacholski, and W. Hans-Joachim. 2010. Effects of free-air $\mathrm{CO}_{2}$ enrichment and nitrogen supply on grain quality parameters and elemental composition of wheat and barley grown in a crop rotation. Agric. Ecosyst. Environ. 136:59-68.

Erdman, J.W., and A.G. Pneros-Schneier. 1994. Factors affecting nutritive value in processed foods. p. 1569-1578. In: M.E. Shils, J.A. Olson, and M. Shiles (eds.), Accounting Principles Fifth Canadian Edition Vol 1 Modern nutrition in health and disease. Lea and Febiger, Philadelphia.

Erenoglu, B., I. Cakmak, V. Römheld, R. Derici, and Z. Rengel. 1999. Uptake of zinc by rye, bread wheat and durum wheat cultivars differing in zinc efficiency. Plant Soil 209:245-252. 
Eyzaguirre, R.Z., K. Nienaltowska, L.E.Q. de Jong, B.B.E. Hasenack, and M.J.R. Nout. 2006. Effect of food processing of pearl millet (Pennisetum glaucum) IKMP-5 on the levels of phenolics, phytate, iron and zinc. J. Sci. Food. Agric. Chem. 86:1391-1398.

Ezzati, M., A.D. Lopez, A. Rodgers, H.S. Vander, and C.J. Murray. 2002. Selected major risk factors and global and regional burden of disease. Lancet 360:1347-1360.

Fageria, N.K., and V.C. Baligar. 2005. Growth components and zinc recovery efficiency of upland rice genotypes. Pesq. Agropec. Bras. Brasilia 40:1211-1215.

Fageria, N.K., V.C. Baligar, and R.B. Clark. 2002. Micronutrients in crop production. Adv. Agron. 77:185-268.

Fageria, N.K., V.C. Baligar, and Y.C. Li. 2008. The role of nutrient efficient plants in improving crop yields in the twenty first century. J. Plant Nutr. 31:1121-1157.

Failla, M.L., S.K. Thakkar, and J.Y. Kim. 2009. In vitro bioaccessibility of $\beta$-carotene in orange fleshed sweet potato (Ipomoea batatas Lam.). J. Agric. Food Chem. 57:10922-10927.

Fairweather-Tait, S., S. Lynch, C. Hotz, R. Hurrell, L. Abrahamse, S. Beeve, S. Bering, K. Bukhave, R. Glahn, M. Hambidge, J. Hunt, B. Lonnerdal, D. Miller, N. Mokhtar, P. Nestel, M. Reddy, A.-S. Sandberg, P. Sharp, B. Teucher, and T:P. Trinidad. 2005. The usefulness of in vitro models to predict the bioavailability of iron and zinc: A consensus statement from the HarvestPlus expert consultation. Int. J. Vitam. Nutr. Res. 75:371-374.

Fairweather-Tait, S., I. Phillips, G. Wortley, L. Harvey, and R. Glahn. 2007. The use of solubility, dialyzability, and Caco-2 cell methods to predict iron bioavailability. Int. J. Vitam. Nutr. Res. 77:158-165.

Fan, M.-S., F.-J. Zhao, S.J. Fairweather-Tait, P.R. Poulton, S.J. Dunham, and S.V. McGrath. 2008. Evidence of decreasing mineral density in wheat grain over the last 160 years. J. Trace Elem. Med. Biol. 22:315-324.

Farnham, M.W., M.A. Grusak, and M. Wang. 2000. Calcium and magnesium concentration of inbred and hybrid broccoli heads. J. Am. Soc. Hort. Sci. 125:344-349.

Fedoroff, N.V., D.S. Battisti, R.N. Beachy, P.J.M. Cooper, D.A. Fischhoff, C.N. Hodges, V.C. Knauf, D. Lobell, B.J. Mazur, D. Molden, M.P. Reynolds, P.C. Ronalds, M.W. Rosegrant, P. A. Sanchez, A. Vonshak, and J.-K. Zhu. 2010. Radically rethinking agriculture for the 21st century. Science 327:833-834.

Fernández-García, E., I. Carvajal-Lérida, and A. Pérez-Gálvez. 2009. In vitro bioaccessibility as a prediction tool of nutritional efficiency. Nutr. Res. 29:751-760.

Feuillet, C., J.E. Leach, J. Rogers, P.S. Schnable, and K. Eversole. 2010. Crop genome sequencing: Lesions and rationals. Trends Plant Sci. 16:77--88.

Ficco, D.B.M., C. Riefolo, G. Nicastro, V. De Simone, A.M. Di Gesù, R. Beleggia, and C. Platani. 2009. Phytate and mineral elements concentration in a collection of Italian durum wheat cultivars. Field Crops Res. 111:235-242.

Fileppi, M., I. Galasso, G. Tagliabue, M.G. Daminati, B. Campion, E. Doria, and F. Sparvoli. 2010. Characterization of structural genes involved in phytic acid biosynthesis in common bean (Phaseolus vulgaris L.). Mol. Breed. 25:453-470.

Flachowsky, G., K. Aulrich, H. Böhme, and I. Halle. 2007. Studies on feeds from genetically modified plants (GMP) - Contributions to nutritional and safety assessment. Anim. Feed Sci. Technol. 133:2-30.

Flyman, M.V. and A.J. Afolayan. 2006. The suitability of wild vegetables for alleviating human dietary deficiencies. S. Afr. J. Bot. 72:492-497.

Forbes, R.M., J.W. Erdman Jr., H.M. Parker; H. Kondo, and S.M. Ketelsen. 1983. Bioavailability of zinc in coagulated soybean protein (tofu) to rats and effects of dietary calcium at a constant phytate:zinc ratio. J. Nutr. 113:205-210. 
Forbes, R.M., H.M. Parker, and J.W. Erdman Jr., 1984. Effect of dietary phytate, calcium, and magnesium levels on zinc bioavailability to rats. J. Nutr. 114:1421-1425.

Fordyce, E.J., R.M. Forbes, K.R. Robbins, and J.W. Erdman Jr., 1987. Phytate $\times$ calcium/zinc molar ratio: Are they predictive of zinc bioavailability? J. Food Sci. 52:440-444.

Frossard, E., M. Bucher, F. Mächler, A. Mozafar, and R. Hurrell. 2000. Potential for increasing the content and bioavailability of $\mathrm{Fe}, \mathrm{Zn}$ and $\mathrm{Ca}$ in plants for human nutrition. J. Sci. Food Agric. 80:861-879.

Fukuda, W.M.G., L. Souza, C.H. Fukuda,. V. Santos, C. Borromeu, M.N. Silva, J.W. Coreolano, J.L. Pinho, and A.R. Santos. 2008. Adopção de variedades de mandioca de polpa amarela para mesa no nordeste brasileiro. Embrapa Mandioca e Fruticultura Tropical, Cruz das Almas, Brasil. Bolentim de Pesquisa e Desenvolvimento no. 41.

Gao, Y., R.M. Biyashev, M.S. Saghai-Maroof, N.M. Glover, D.M. Tucker, and G.R. Buss. 2008. Validation of low phytate QTLs and evaluation of seedling emergence of low phytate soybeans. Crop Sci. 48:1355-1364.

Gao, X.P., C.Q. Zou, X.Y. Fan, F.S. Zhang, and E. Hoffland. 2006. From flooded to aerobic conditions in rice cultivation: Consequences for zinc uptake. Plant Soil 280:41-47.

García-Casal, M.N., M. Layrisse, L. Solano, M.A. Barón, F. Arguello, D. Llovera, J. Ramírez, I. Leets, and E. Tropper. 1998. Vitamin $A$ and $\beta$-carotene can improve nonheme iron absorption from rice, wheat and corn by humans. J. Nutr. 128:646-650.

García del Moral, L.F., Y. Rharrabti, D. Villegas, and C. Royo. 2003. Evaluation of grain yield and its components in durum wheat under Mediterranean conditions: An ontogenic approach. Agron. J. 95:266-274.

Garcia-Oliveira, A.L., L. Tan, Y. Fu, and C. Sun. 2009. Genetic identification of quantitative trait loci for contents of mineral nutrients in rice grain. J. Integr. Plant Biol. 51:84-92.

Garrett, D.A., M.L. Falla, and R.J. Sarama. 1999. Development of an in vitro digestion method to assess carotenoid bioavailability from meals. J. Agric. Food Chem. 47:43014309 .

Garrido, S.M., C.K. Morikawa, H. Nakanishi, and M. Saigusa. 2006. Strategies on iron mobilization and uptake in plant roots. Tohoku J. Agric. Res. 56:21-35.

Garvin, D.F., R.M. Welch, and J.W. Finley. 2006. Historical shifts in seed mineral micronutrient concentration of US hard red winter wheat germplasm. J. Sci. Food Agric. $86: 2213-2220$.

Gautam, S., K. Platel, and K. Srinivasan. 2010a. Higher bioaccessibility of iron and zinc from food grains in the presence of garlic and onion. J. Agric. Food Chem. $58: 8426-8429$.

Gautam, S., K. Platel, and K. Srinivasan. 2010b. Influence of $\beta$-carotene-rich vegetables on the bioaccessibility of zine and iron from food grains. Food Chem. 122:668-672.

Gelin, J.R., S. Forster, K.F. Grafton, P.E. McGlean, and G.A. Rojas-Cifuentes. 2007. Analysis of seed zinc and other minerals in a recombinant inbred population of Navy bean (Phaseolus vulgaris L.). Crop Sci. 47:1361-1366.

Genc, Y., and G.K. McDonald. 2008. Domesticated emmer wheat [T. turgidum L. subs. dicoccon (Schrank) Thell.] as a source for improvement of zinc efficiency in durum wheat. Plant Soil 310:67-75.

Genc, Y., A.P. Verbyla, A.A. Torun, I. Cakmak, K. Willsmore, H. Wallwork, and G.K. McDonald. 2009. Quantitative trait loci analysis of zinc efficiency and grain zinc concentration in wheat using whole.genome average interval mapping. Plant Soil $314: 49-66$.

Ghandilyan, A., D. Vreugdenhil, and M.G.M. Aarts. 2006. Progress in the genetic understanding of plant iron and zinc nutrition. Physiol, Plant 126:407-417. 
Glahn, R.P., Z. Cheng, R.M. Welch, and G.B.A. Gregorio, 2002. Comparison of iron bioavailability from 15 rice genotypes: Studies using an in vitro digestion/Caco-2 cell culture model. J. Agric. Food Chem. 50:3586-3591.

Glahn, R., and O. Hoekenga. 2011. Identification of iron bioavailability QTL in maize. Symposia Brief: Breeding for Bioavailability. First Global Conference on Biofortification. November 9-11, Washington, DC.

Glahn, R.P., C. Lai, J. Hsu, J.F. Thompson, and D.R. Van Campen, 1998a. Decrease citrate improves iron availability from infant formula: Application of an in vitro digestion/Caco-2 cell culture model. J. Nutr. 128:257-264.

Glahn, R.P., O.A. Lee, A. Yeung, M.I. Goldman, and D.D. Miller. 1998b. Caco-2 cell ferritin formation predicts nonradiolabeled food iron availability in an in vitro digestion/Caco-2 culture model. J. Nutr. 128:1555-1561.

Glahn, R.P., E.M. Wein, D.R. Van Campen, and D.D. Miller. 1996. Caco-2 cell iron uptake from meat and casein digests parallels in vivo studies: Use of a novel in vitro method for rapid estimation of iron bioavailability. J. Nutr, 126:332-339.

Gómez-Becerra, H.F., A. Yazici, L. Ozturk, H. Budak, Z. Peleg, A. Morgounov, T. Fahima, Y. Saranga, and I. Cakmak; 2010. Genetic variation and environmental stability of grain mineral nutrient concentrations in Triticum dicoccoides under five environments. Euphytica 171:39-52.

Gómez-Galera, S., E. Rojas, D. Sudhakar, C. Zhu, A.M. Pelacho, T. Capell, and P. Christou. 2010. Critical evaluation of strategies for mineral fortification of staple food crops. Transgenic Res. 19:165-180.

González, B., J.I. Baños-Sanz, M. Villate, C.A. Brearley, and J. Sanz-Aparicio. 2010. Inositol 1,3,4,5;6- pentakisphosphate 2-kinase is a distant IPK member with a singular inositide binding site for axial 2-OH recognition. Proc. Natl. Acad. Sci. USA 107:96089613.

González, C., N. Johnson, and M. Qaim. 2009. Consumer acceptance of second-generation GM foods: The case of biofortified cassava in the north-east of Brazil. J. Agric. Econ. 60:604-624.

González, C., S. Perez, C.E. Cardoso, R. Andrade, and N. Johnson. 2011. Analysis of diffusion strategies in northeast Brazil for new cassava varieties with improved nutritional quality. Exp. Agric. 47:539-552.

Goto, F., T. Yoshihara, N. Shigemoto, S. Toki, and F. Takaiwa. 1999. Iron fortification of rice seed by the soybean ferritin gene. Nat. Biotechnol. 17:282-286.

Gourley, C.J.P., D.L. Allan, and M.P. Russelle. 1994. Plant nutrient efficiency: A comparison of definitions and suggested improvement. Plant Soil 158:29-37.

Graham, R.D., D. Senadhira, S. Beebe, C. Iglesias, and I. Monasterio. 1999. Breeding for micronutrient density in edible portions of staple food crops: Conventional approaches. Field Crops Res. 60:57-80.

Graham, R.D. and R.M. Welch. 1996. Breeding for staple food crops with high micronutrient density. Working papers on agricultural strategies for micronutrients, no. 3. Washington, DC: International Food Policy Research Institute.

Graham, R.D., R.M. Welch, D.A. Saunders; I. Ortiz-Monasterio, H.E. Bouis, M. Bonierbale, S. de Haan, G. Burgos, G. Thiele, R. Liria, C.A. Meisner, S.E, Beebe, M.J. Potts, M. Kadian, P.R. Hobbs, R.K. Gupta, and S. Twomlow. 2007. Nutritious subsistence food systems. Adv. Agron. 92:1-74.

Granado-Lorencio, F., B. Olmedilla-Alonso, C. Herrero-Barbudo, B. Pérez-Sacristán, I. Blanco-Navarro, and S. Blázquez-Garcia. 2007. Comparative in vitro bioaccessibility of carotenoids from relevant contributors to carotenoid intake. J. Agric. Food Chem. $55 ; 6387-6394$. 
Grata, E., D. Guillarme, G. Glauser, J. Boccard, P.A. Carrupt, J.L. Veuthey, S. Rudaz, and J.L. Wolfender. 2009. Metabolite profiling of plant extracts by ultra-high pressure liquid chromatography at elevated temperature coupled to time-of-flight mass spectrometry. J. Chromatogr. 1216:5660-5668.

Gregor, J.L. 1992. Using animals to assess bioavailability of minerals: Implications for human nutrition. J. Nutr، 122:2047-2052.

Gregorio, G.B., D. Senadhira, H. Htut, and R.D. Graham. 2000. Breeding for trace mineral density in rice. Food Nutr. Bull. 21:382-386.

Groote, H.D., and S.C. Kimenju. 2008: Comparing consumer preferences for color and nutritional quality in maize: Application of a semi-double-bound logistic model on urban consumers in Kenya. Food Policy 33:362-370.

Grotz, N., and M.L. Guerinot. 2006. Molecular aspects of Cu, Fe and Zn homeostasis in plants. Biochim. Biophys. Acta 1763:595-608.

Grusak, M.A. 1994. Iron transport to developing ovules of Pisum sativum. I. Seed import characteristics and phloem iron-loading capacity of source regions. Plant Physiol. 104:649-655.

Grusak, M.A. 1995. Whole-root iron(III)-reductase activity throughout the life cycle of iron-grown Pisum sativum L. (Fabaceae): Relevance to the iron nutrition of developing seeds. Planta 97:111-117.

Grusak, M.A. 2000. Strategies for improving the iron nutritional quality of seed crops: Lesions learned from the study of unique iron-hyperaccumulating pea mutants. Pisum Genet. 32:1-5.

Grusak, M.A. 2002. Enhancing mineral content in plant food products. J. Am. Coll. Nutr. 21:178S-183S.

Guerinot, M.L. 2001. Improving rice yields-Ironing out the details. Nat. Biotechnol. 19:417-418.

Gunratna, N.S., H. de Groote, P. Nestel, K.V. Pixley, and G.P. McCabe. 2010. A meta-analysis of community-based studies on quality protein maize. Food Policy 35:202-210.

Gupta, S.K., G. Velu, K.N. Rai, and K. Sumalini. 2009. Association of grain iron and zinc content with grain yield and other traits in pearl millet (Pennisetum galucum (L.) R. BR.). Crop Improv, 36:4-7.

Guttieri, M., D. Bowen, J.A. Dorsch, V. Raboy, and E. Souza. 2004. Identification and characterization of a low phytic acid wheat. Crop Sci. 44:418-424.

Guzmán-Maldonado, S.H., J. Acosta-Gallegos, and O. Paredes-López. 2000. Protein and mineral content of a novel collection of wild and weedy common bean (Phaseolus vulgaris L.). J. Sci. Food Agric. 80:1874-1881.

Guzmán-Maldonado, S.H., O. Martínez, J.A. Acosta-Gallegos, F. Guevara-Lara; and O. Paredes-López. 2003. Putative quantitative trait loci for physical and chemical components of common bean. Crop Sci. 43:1029-1035.

Haas, J.D., J.L. Bear, L.E. Murray-Kolb, A.M. Del Mundo, A. Felix, and G.B. Gregorio. 2005. Iron biofortified rice improves the iron stores of non-anemic Filippino women. J. Nutr. 135:2823-2830.

Hacisalihoglu, G., L.V. Kochian, and C.E. Vallejos. 2005. Distribution of seed mineral nutrients and their correlation in Phaseolus vulgaris. Proc. Fla. State Hort. Sci. 118:102105.

Haider, J., A.M. Omwega, N.M. Muroki, and G. Ayana. 2003. Daily versus weekly iron supplementation and prevention of iron deficiency anemia in lactating women. East Afr. Med. J. 80:11-16. 
Hao, H., Y. Wei, X. Yang, Y. Feng, and C. Wu. 2007. Effects of different nitrogen fertilizer levels on $\mathrm{Fe}, \mathrm{Mn}, \mathrm{Cu}$ and $\mathrm{Zn}$ concentrations in shoot and grain quality in rice (Oryza sativa). Rice Sci. 14:289-294.

Harjes, C.E., T.R. Rocheford, L. Bai, T.P. Brutnell, C.B. Kandianis, S.G. Sowinski, A.E. Stapleton, R. Vallabhaneni, M. Williams, E.T. Wurtzel, J. Yan, and E.S. Buckler. 2008. Natural genetic variation in Lycopene epsilon cyclase tapped for maize biofortification. Science 319:330-333.

Haryu, Y., Y. Taguchi, E. Itakura, O. Mikami, K. Miura, T. Saeki, and Y. Nakajima. 2009. Longterm biosafety assessment of a genetically modified (GM) plant: The genetically modified (GM) insect-resistant Bt11 corn does not affect the performance of multigenerations or life span of mice. Open Plant Sci. J. 3:49-53.

Hash, C.T., A.G. Bhaskar Raj, S.A. Appa Rao, and U. Singh. 1997. New sources of yellow endosperm and $\beta$-carotene in pearl millet. Proc. Int. Conf. Genet. Improv. Sorghum and Pearl Millet, September 22-27, 1996. Holiday Inn Plaza, Lubboack, Texas, Lincoln, NE, USA:INTSORMIL.

Haslett, B.S., R.J. Reid, and Z. Rengel, 2001. Zinc mobility in wheat: Uptake and distribution of zinc applied to leaves or roots. Ann. Bot. 87:379-386.

He, X., and K. Nara. 2007. Element biofortification: Can mycorrhizas potentially offer a more effective and sustainable pathway to curb human malnutrition? Trends Plant Sci. 12:331-333.

Hell, R. and U.W. Stephan. 2003. Iron uptake, trafficking and homeostasis in plants. Planta 216:541-551.

Hemalatha, S., K. Platel, and K. Srinivasan 2007a. Influence of germination and fermentation on the bioaccessibility of zinc and iron from food grains. Eur. J. Clin. Nutr. 16:342-348.

Hemalatha, S., K. Platel, and K. Srinivasan. 2007b. Influence of heat processing on the bioaccessibility of zinc and iron from cereals and pulses consumed in India. J. Trace. Elem. Med. Biol. 21:1-7.

Herren, T, , and U. Feller. 1994. Transfer of zinc from xylem to phloem in the peduncle of wheat. J. Plant Nutr. 17:1587-1598.

Hirschi, K.D. 2009. Nutritional biofortification of food crops. Ann. Rev. Nutr. 29:401-421.

Högy, P. and A. Fangmeier, 2008. Effects of elevated atmospheric $\mathrm{CO}_{2}$ on grain quality of wheat. J. Gereal Sci. 48:580-591.

Högy, P., J. Franzaring, K. Schwadorf, J. Breuer, W. Schultze, and A. Fangmeier. 2010. Effect of free-air $\mathrm{CO}_{2}$ enrichment on energy traits as seed quality of oilseed rape. Agric. Ecosyst. Environ. 139:239-244,

Högy, P., H. Wieser, P. Köhler, K. Schwadorf, J. Breuer, J. Franzaring, R. Muntifering, and A. Fangmeier. 2009. Effect of elevated $\mathrm{CO}_{2}$ on grain yield and quality of wheat: Results from a 3-year free-air $\mathrm{CO}_{2}$ enrichment experiment. Plant Biol. 11 (Suppl. 1). 60-69.

Hokanson, K.E., N.C. Ellstrand, J.T. Ouedraogo, P.A. Olwney, B.A. Schaal, and A.F. Raybould. 2010. Biofortified sorghum in Africa; Using problem formulation to inform risk assessment. Nat. Biotechnol. 28:900-903.

Hotz, C., and R.S. Gibson. 2007. Traditional food processing and preparation practices to enhance the bioavailability of micronutrients in plant-based diets. J. Nutr. 137:1097-1100.

Howe, J.A., and S.A. Tanumihardjo. 2006. Carotenoid-biofortified maize maintains adequate vitamin A status in Mongolian gerbils. J. Nutr. 136:2562-2567.

Hu, Y., Z. Cheng, L. Heller, S. Krasnoff, R. Glahn, and R. Welch. 2006. Kaempferol and quercetrin effect on iron bioavailability in white and colored bean seeds (Phaseolus vulgaris L.) using an in vitro digestion/human Caco-2 cell model. FASEB J. 20:A197. 
Hulshof, P.J.M., T. Kosmeijer-Schuil, C.E. West, and P.C.H. Hollman. 2007. Quick screening of maize kernels for provitamin A content. J. Food Comp. Anal. 20:655-661.

Hurrell, R., and I. Egli. 2010. Iron bioavailability and dietary reference values. Am. J. Clin. Nutr. 91:1461S-1467S.

Husted, S., D.P. Persson, K.H. Laursen, T.S. Hansen, P. Pedas, M. Schiller, J.N. Hegelind, and J.K. Schjoerring. 2011. Review: The role of atomic spectrometry in plant science. J. Anal. At. Spectrom. 26:52-79.

ICRISAT. 2010. ICRISAT Archival report 2009: MTP Project 5-Producing more and better food at lower cost of staple cereal and legume hybrids in the Asian SAT (sorghum, pearl millet and pigeonpea) through genetic improvement. ICRISAT, Patancheru, India.

Imhoff-Kunsch, B., R. Flores, O. Dary, and R. Martorell. 2007. Wheat flour fortification is unlikely to benefit the neediest in Guatemala. J. Nutr. 137:1017-1022.

Imtiaz, M., J.B. Alloway, A.K. Shah, S.H. Siddiqui, M.Y. Memon, M. Aslam, and P. Khan. 2003. Zinc contents in the seed of some domestic and exotic wheat genotypes. Asian J. Plant Sci. 2:1118-1120.

IRGSP. 2005. The map-based sequence of rice genome. Nature 436:793-800.

Islam, F.M.A., K.E. Basford, C. Jara, R.J. Redden, and S.E. Beebe. 2002. Seed compositional and disease resistance differences among gene pools in cultivated common bean. Genet. Resour. Crop Evol. 49:285-293.

Islam, F.M.A., S.E. Beebe, M. Muñoz, J. Tohme, R.J. Redden, and K.E. Basford. 2004. Using molecular markers to assess the effect of introgression on quantitative attributes of common bean in the Andean gene pool. Theor. Appl. Genet. 108:243-252.

Israel, D.W., P. Kwanyuen, and J.W. Burton. 2006. Genetic variation for phytic acid phosphorus and inorganic phosphorus in seeds of soybean maturity groups V, VI, and VII. Crop Sci. 46:67-71.

James, C. 2008. Global status of commercialized biotech/GM crops 2008. ISAAA Briefs No. 39. ISSAAA, Ithaca, NewYork.

Jansen van Rensburg, W.S., S.L. Venter, T.R. Netshiluvhi, E. Van den Heever, H.J. Vorster, and J.A. de Ronde. 2004. Role of indigenous leafy vegetables in combating hunger and malnutrition. South Afr. J. Bot. 70:52-59.

Jarrell, W.M., and R.B. Beverly. 1981. The dilution effect in plant nutrition studies. Adv. Agron. 34:197-224.

Jiang, S., C. Shi, and J. Wu. 2009. Studies on mineral nutrition and safety of wild rice (Oryza L.). Int. J. Food Sci. Nutr, 60:139-147.

Jiang, W., P.C. Struik, H. van Keulen, M. Zhao, and T.J. Stomph. 2008. Does increased zinc uptake enhance grain zinc mass concentration in rice? Ann. Appl. Biol. 153:135-147.

Jiang, X., X. Wu, and G. Xiao. 2010. Effect of genetically modified herbicide-tolerant (GMHT) rice on biodiversity of weed in paddy fields. Afr. J. Biotechnol. 9:3122-3130.

Joshi, A.K., J. Grossa, B. Arun, R. Chand, R. Trethowan, M. Vargas, and I. Ortiz-Monasterio. 2010. Genotype $\times$ environment interaction for zinc and iron concentration of wheat grain in eastern Gangetic plain of India. Field Crops Res. 116:268-277.

Kalgaonkar, S., and B. Lönnerdal. 2008. Effects of dietary factors on iron uptake from ferritin in Caco-2 cells. J. Nutr. Biochem, 19:33-39.

Kandlakunta, B., A. Rajendran, and L. Thingnganing. 2008. Carotene content of some common (cereals, pulses, vegetables, spices and condiments) and unconventional sources of plant origin. Food Chem. 106:85-89.

Kaur, N., K. Street, M. Mackay, N. Yahiaoui, and B. Keller, 2008. Molecular appróaches for characterization and use of natural disease resistance in wheat. Eur. J. Plant Pathol. 121:387-397. 
Kayodé, A.P.P., A.R. Limemann, J.D. Hounhouigan, M.J. Nout, and M.A.J.S. van Boekel. 2006. Genetic and environmental impact on iron, zinc and phytate in food sorghum grown in Benin. J. Agric. Food Chem. 54:256-262.

Kean, E.G., B.R. Hamaker, and M.G. Ferruzzi. 2008. Carotenoid bioaccessibility from whole grain and degermed maize meal products. J. Agric. Food Chem, 56:9918-9926.

Khalekuzzaman, M., K. Datta, N. Oliva, M.F. Alam, O.I. Joarder, and S.K. Datta. 2006. Stable integration, expression and inheritance of the ferritin gene in transgenic elite indica rice cultivar BR29 with enhanced iron level in the endosperm. Indian J. Biotechnol. 5:26-31.

Khangura, B.S., K.S. Gill, and P.S. Phul. 1980. Combining ability analysis of beta-carotene, total carotenoids and other grain characteristics in pearl millet. Theor. Appl. Genet. 56:91-96.

Khoshgoftarmanesh, A.H., R. Schulin, R.L. Chaney, B. Daneshbakhsh, and M. Afyuni. 2010. Micronutrient-efficient genotypes for crop yield and mutritional quality in sustainable agriculture: A review. Agron. Sustain. Dev. 30:83-107.

Kim, S.I., G.B. Andaya, J.W. Newman, S.S. Goyal, and T.H. Tai. 2008. Isolation and characterization of a low phytic acid rice mutant reveals a mutation in the rice orthologue of maize MIK. Theor. Appl. Genet. 117:1291-1301.

Kimenju, S.C. and H.D. Groote. 2008. Consumers' willingness to pay for genetically modified foods in Kenya. Agric. Econ, 38:35-46.

Kobayashi, T., H. Nakanishi, S. Mori, and N. Nishizawa. 2008. Generation and field trials of transgenic rice tolerant to iron deficiency. Rice 1:144-153.

Kobayashi, T., and N.K. Nishizawa. 2008. Regulation of iron and zinc uptake and translocation in rice. p.321-335. In: H.Y. Hirano, A. Hirai, Y. Sano, and T. Sasaki (eds.), Accounting Principles Fifth Canadian Edition Vol 1 Biotechnology in agriculture and forestry 62 rice biology in the genomics era. Springer, Berlin.

Koegel-Knabner, I., W. Amelung, Z. Cao, S. Fieldler, P. Frenzel, R. Jahn, K. Kalbitz, A. Koelbl, and M. Schloter. 2010. Biogeochemistry of paddy soils. Geoderma 157:1-14.

Krishnan, S., and P. Dayanandan: 2003. Structural and histochemical studies on grain filling in the caryopsis of rice (Oryza sativa L.). J. Biosci. 28:455-469.

Kristensen, C., M. Morant, C. Eoleson, C.T. Ekstrøm, D.W. Galbraith, B.L. Moller, and S. Bak. 2005. Metabolic engineering of dhurrin in transgenic Arabidopsis plants with marginal inadvertent effects on metablome and transcriptome. Proc. Natl. Acad. Sci. USA 102:1779-1784.

Krivanek, A.F., H. de Groote, N.S. Gunratna, A.O. Diallo, and D. Friesen. 2007. Breeding and disseminating quality protein maize (QPM) for Africa. Afr. J. Biotechnol. 6:312324 ,

Kumar, A.A., B.V.S. Reddy, B. Ramaiah, P.S. Reddy, K.L. Sahrawat, and H.D. Upadhyaya. 2009. Genetic variability and plant character association of grain $\mathrm{Fe}$ and $\mathrm{Zn}$ in sèlected core collection accessions of sorghum germplasm and breeding lines. SAT J. 7.

Kumar, A.A., B.V.S. Reddy, K.L. Sahrawat, and B. Ramaiah. 2010a. Combating micronutrient malnutrition: Identification of commercial sorghum cultivars with high grain iron and zinc. SAT J. 8.

Kumar, S., T. Nepolean, K.N. Rai, V. Rajaram, G. Velu, K.L. Sahrawat, P.V. Rao, A.S. Rao, S. Senthilvel, A. Ganapathi, G. Singh, and C.T. Hash. 2010b. Mapping pearl millet [Pennisetum glaucum (L.) R. Br.] QTLs for $\mathrm{Fe}$ and $\mathrm{Zn}$ grain density. Poster 250. 2nd Int. Symp. Genom. Plant Genet. Resour., April 24-27, 2010, Bologna, Italy.

Kutman, U.B., B. Yildiz, L. Ozturk, and I. Cakmak, 2010. Biofortification of durum wheat with zinc through soil and foliar applications of nitrogen. Cereal Chem. 87:1-9. 
Kuwano, M., T. Mimura, F. Takaiwa, and K.T. Yoshida. 2009. Generation of stable low phytic acid transgenic rice through antisense repression of the 1D-myo-inositol 3-phosphate synthase gene (RINO1) using the 18-kDa oleosin promoter. Plant Biotechnol. 7:96-105.

Kuwano, M., A. Ohyama, Y. Tanaka, T. Mimura, F. Takaiwa, and K.T. Yoshida. 2006. Molecular breeding for transgenic rice with low-phytic acid phenotype through manipulating myo-inositol 3-phosphate synthase gene. Mol. Breed, 18:263-272.

Kwaku, D., and A. Asante. 2008. Genetically modified food: The dilemma of Africa. Afr. ). Biotechnol. 7:1204-1211.

Landau-Ellis, D., and V.R. Pantalone. 2009. Marker-assisted backcrossing to incorporate two low phytate alleles into Tennessee soybean cultivar 5601T. p. 316-318. In Q.Y. Shu (ed.), Accounting Principles Fifth Canadian Edition Vol 1 Induced plant mutations in the genomics era. Food and Agricultural Organization of the United Nations, Rome, Italy.

Lappé, M.A., E.B. Bailey, C. Chandra, and K.D. Setchell. 1999. Alterations in clinically important phytoesterogens in genetically modified, herbicide tolerant soybeans. J. Med. Food 1:241-245.

Larson, S.R. J.N. Rutger, K.A. Young, and V. Raboy. 2000. Isolation and genetic mapping of a non-lethal rice (Oryza sativa L.) low phytic acid 1 mutation. Crop Sci. 40:1397-1405.

Laszlo, J.A. 1990. Mineral contents of soybean seed coats and embryas during development. J. Plant Nutr. 13:231-248.

Layrisse, M., M.N. García-Casal, L. Solano, F. Arguella, D. Llovera, J. Romírez, I. Leets, and E. Tropper. 2000. New property of vitamin A and $\beta$-carotene on human iron absorption: Effect on phytate and polyphenols as inhibitors of iron absorption. Arch. Latinoam. Nutr. 50:243-248.

Lee, C.M. , A.C. Boileau, T.W.M. Boileau, A.W. Williams, K.S. Swanson, K. A Heintz, and J.W. Erdman Jr., 1999. Review of animal models in carotenoids research. J. Nutr. 129:2271-2277.

Lee, S., U.S. Jeon, S.J. Lee, Y.-K. Kim, D.P. Persson, S. Husted, J.K. Schjørring, Y. Kakei, H. Masuda, N.K. Nishizawa, and G. An. 2009. Iron fortification of rice seeds through activation of the nicotianamine synthase gene. Proc. Natl. Acad. Sci. USA 106:2201422019.

Lee, C.M., J.D. Lederman, N.E. Hoffmann, and J.W. ErdmanJr., 1998. The Mongolian gerbil (Meriones unguiculatus) is an appropriate animal model for evaluation of the conversion of $\beta$-carotene to vitamin A. J. Nutr. 128:280-286.

Li, Q., G. Farre, S. Naqvi, J. Breitenbach, G. Sanahuja, C. Bai, G. Sandmann, T. Capell, P. Christou, and C. Zhu. 2010a. Cloning and functional characterization of the maize carotenoids isomerase and $\beta$-carotene hydroxylase genes and their regulation during endosperm maturation. Transgenic Res. 19:1053-1068.

Li, S., A. Nugroho, R. Rochefrdm, and W.S. White. 2010b. Vitamin A equivalence of the $\beta$-carotene in $\beta$-carotene-biofortified maize porridge consumed by women. Am. J. Clin. Nutr. 92:1105-1112.

Li, S., F.A.K. Tayie, M.F. Young, T. Rocheford, and W.S. White. 2007. Retention of provitamin $A$ carotenoids in high $\beta$-carotene maize (Zea. mays) during traditional African household processing. J. Agric. Food Chem. 55:10744-10750.

Liu, W. 2010. Do genetically modified plants impact arbuscular mycorrhizal fungi? Ecotoxicology 19:229-238.

Liu, C.-S., R.P. Glahn, and R.H. Liu. 2004. Assessment of carotenoids biodvailability of whole foods using a Caco-2 cell culture model coupled with an in vitro digestion. J. Agric. Food Chem. 52:4330-4337. 
Liu, Q.-L., X.-H. Xu, X.-L. Ren, H.-W. Fu, D.-X. Wu, and Q.-Y. Shu. 2007. Generation and characterization of low phytic acid germplasm in rice (Oryza sativa L.). Theor. Appl. Genet. 114:803-814.

Lolas, G.M. and P. Markakis. 1975. Phytic acid and other phosphorus compounds of beans (Phaseolus vulgaris L.). J. Agric. Food Chem, 23:13-15.

Lombi, E., K.G. Scheckel, and I.M. Kempson. 2010. In situ analysis of metal(loid)s in plants: State of the art and artifacts. Environ. Expt. Bot. 72:3-17.

Lombi, E., K.G. Scheckel, J. Pallon, A.M. Carey, Y.G. Zhu, and A.A. Meharg. 2009. Speciation and distribution of arsenic and localization of nutrients in rice grains. New Phytol. 184:193-201.

Lombi, E., E. Smith, T.H. Hansen, D. Paterson, M.D. de Jonge, D.L. Howard, D.P. Persson, S. Husted, C. Ryan, and J.K. Schjoerring. 2011. Megapixel imaging of (micro)nutrients in mature barley grains. J. Expt. Bot. 62:273-282.

Lonergan, P.F, M.A. Pallotta, M. Lorimer, J.G. Paull, S.J. Barker, and R.D. Graham. 2009. Multigenic loci for zinc uptake and distribution in barley (Hordeum valgare). New Phytol. 184:168-179.

Lönnerdal, B. 2003. Genetically modified plants for improved trace element nutrition. J. Nutr. 133:1490S-1493S.

Lorenz, A.J., M.P. Scott, and K.R. Lamkey. 2007. Quantitative determination of phytate and inorganic phosphorous for maize breeding. Crop Sci. 47:600-606.

Lott, J.N.A., I. Ockenden, V. Raboy, and G.D. Batten. 2000. Phytic acid and phosphorus in crop seeds and fruits: A global estimate. Seed Sci. Res. 10:11-33.

Low, J., M. Arimond, N. Osman, B. Cunguara, F. Zano, and D. Tschirley. 2007. A food-based approach introducing orange-fleshed sweetpotato increased vitamin $A$ intake and serum retinol concentrations in young children in rural Mozambique. J. Nutr. 137:1320-1327.

Lowe, N.M., K. Fekete, and T. Decsi. 2009. Methods of assessment of zinc status in humans; A systematic review. Am. J. Clin. Nutr. 89:2040S-2051S.

Lu, S., and L. Li. 2008. Carotenoid metabolism: Biosynthesis, regulation, and beyond. J. Integr. Plant Biol. 50:778-785.

Lucca, P., R. Hurrell, and I. Potrykus. 2001. Genetic engineering approaches to improve the bioavailability and the level of iron in rice grains. Theor. Appl. Genet, 102:392-397.

Lucca, P., R. Hurrell, and I. Potrykus. 2002. Fighting iron deficiency anemia with iron-rich rice. J. Am. Coll. Nutr. 21:184S-190S.

Lung'aho,. M.G., and R.P. Glahn. 2010. Use of white beans instead of red beans may improve iron bioavailability from a Tanzanian complementary food mixture. Int. J. Vitam. Nutr. Res. 80:24-31.

Lynch, J.P. 2007. Roots of the second green revolution. Aust. J. Bot. 55:493-512.

Lyons, G.H., Y. Genc, J.C.R. Stangoulis, L.T. Palmer, and R.D. Graham. 2005. Selenium distribution in wheat grain, and the effect of postharvest processing on wheat selenium content. Biol. Trace Elem. Res. 103:155-168.

Ma, G., Y. Jin, Y. Li, F. Zhai, F.J. Kok, E. Jacobsen, and X. Yang, 2007, Iron and zinc deficiencies in China: What is feasible and cost-effective strategy? Public Health Nutr. 11:632-638.

Magaña-Gómez, J.A., G.L. Cervantes, G. Yepiz-Plascencia, and A.M.C. de la Barca. 2008. Pancreatic response of rats fed genetically modified soybean. J. Appl. Toxicol. 28:217-226.

Magaña-Gómez, J.A., and A.M.C. de la Barca. 2008. Risk assessment of genetically modified crops for nutrition and health. Nutr. Rev, 67:1-16.

Maman, N., S.C. Mason, D.J. Lyon, and P. Dhungana. 2004. Yield components of pearl millet and grain sorghum across environments in the central Great Plains. Crop Sci. $44 \vdots 2138-2145$. 
Mamatha, B.S., R. Arunkumar, and V. Baskaran. 2010. Effect of processing on major carotenoids levels in corn (Zea mays) and selected vegetables: Bioavailability of lutein and zeaxanthin from processed corn in mice. Food Bioproc. Technol. 5(4):1355-1363.

Marschner, H. 1995. Accounting Principles Fifth Canadian Edition Vol 1 Mineral nutrition of higher plants. Academic Press, London, UK.

Marschner, H., V. Romheld, and M. Kissel. 1986. Different strategies in higher plants in mobilization and uptake of iron. J. Plant Nutr. 9:695-713.

Masuda, H., M. Suzuki, K.C. Morikawa, T. Kobayashi, H. Nakanishị, M. Takahashi; M. Saigusa, S. Mori, and N.K. Nishizawa, 2008. Increase in iron and zinc concentrations in rice grains via the introduction of barley genes involved in phytosiderophore synthesis. Rice 1:100-108.

Masuda, H., K. Usuda, T. Kobayashi, Y. Ishimaru, Y. Kakei, M. Takahashi, K. Higuchi, H. Nakanishi, S. Mori, and N.K. Nishizawa. 2009. Overexpression of the barley nicotianamine synthase gene HVNAS1 increases iron and zinc concentrations in rice grains. Rice 2:155-166.

Mazariegos, M., K.M. Hambidge, N.F. Krebs, J.E. Westcott, S. Lei, G.K. Grunwald, R. Campos, B. Barahona, V. Raboy, and N.W. Solomons. 2006. Zinc absorption in Guatemalan schoolchildren fed normal or low-phytate maize. Am. J. Clin. Nutr. 83:59-64.

Mazzolini, A.P., C.K. Pallaghy, and G.J.F. Legge. 1985. Quantitative microanalysis of Mn, $\mathrm{Zn}$ and other elements in mature wheat seed. New Phytol. 100:483-509.

McRae, R., P. Bagchi, S. Sumalekshmy, and C.J. Fahrni. 2009. In situ imaging of metals in cell and tissues. Chem. Rev. 109:4780-4827.

Meenakshi, J.V., N.L. Johnson, V.M. Manyong, H. Degroote, J. Javelosa, D.R. Yanggen, F. Naher, C. Gonzalez, J. García, and E. Meng. 2010. How cost-effective is biofortification in combating micronutrient malnutrition? An ex ante assessment. World Develop. 38:6475.

Mei, Z., M.E. Cogswell, I. Parvanta, S. Lynch, J.L. Beard, R.J. Stoltzfus, and L.M. GrummerStrawn. 2005. Hempglobin and ferritin are currently the most efficient indicators of population response to iron interventions: An analysis of nine randomized controlled trials. J. Nutr. 135:1974-1980.

Meis, S.J., W.R. Fehr, and S.R. Schnebly. 2003. Seed source effect on field emergence of soybean lines with low phytate. Crop Sci. 43:1336-1339.

Menkir, A., W. Liu, W.S. White, B. Maziya-Dixon, and T. Rocheford. 2008. Carotenoid diversity in tropical-adapted yellow maize inbred lines, Food Chem. 109:521-529.

Menkir, A., and B. Maziya-Dixon. 2004. Influence of genotype and environment on $\beta-$ carotene content of tropical yellow-endosperm maize genotypes. Maydica 49:313-318.

Mihashi, S., and S. Mori. 1989. Characterization of mugineic acid-Fe transporter in Fedeficient barley roots using the multicompartment transport box method. Biometals 2:146-154.

Miller, L.V., N.F. Krebs, and K.M. Hambidge. 2007. A mathematical model of zinc absorption in humans as a function of dietary zinc and phytate. J. Nutr. 137:135-141.

Mills, H.A., and J.B. Jones Jr. 1996. Accounting Principles Fifth Canadian Edition Vol 1 Plant analysis handbook II. A practical sampling, preparation, analysis, and interpretation guide. Micro-Macro Publishing, Athens, GA, USA.

Minekus, M., P. Marteau, R. Havenaar, and J.H.J. Huis in't Veld. 1995. A multicompartmental dynamic computer-controlled model simulating the stomach and small intestine. ATLA 23:197-209.

Misawa, N., M. Nakagawa, K. Kobayashi, S. Yamano, Y. Izawa, K. Nakamura, and K. Harashima. 1990. Elucidation of Erwinia uredovora carotenoids biosynthetic pathway 
by functional analysis of gene products expressed in Escherichia coli. J. Bacteriol. 172:6704-6712.

Monsen, E.R., L. Hallberg, M. Layrisse, D.M. Hegsted, J.D. Cook, W. Mertz, and C.A. Finch. 1978. Estimation of available dietary iron. Am. J. Clin. Nutr. 31:134-141.

Moore, K.L., M. Schröder, E. Lombi, F.J. Zhao, S.P. McGrath, M.J. Hawkesford, P. Shewry, and C.R.M. Grovenor. 2010. NanoSIMS analysis of arsenic and selenium in cereal grains. New Phytol. 185:434-445.

Moraes, M.F., C.H. Abreu Jr, J.J. Hart, R.M. Welch, and L.V. Kochian. 2010. Genotypic variation in micronutrient concentrations in grain of 35 upland rice cultivars. 19th World Congress of Soil Science, Soil Solutions for a Changing World, 1-6 August 2010, Brisbane, Australia.

Moraghan, J.T. 2004. Accumulation and within-seed distribution of iron in common bean and soybean. Plant Soil 264:287-297.

Moraghan, J.T., and K. Grafton, 2002. Distribution of selected elements between the seed coat and embryo of two black bean cultivars. J. Plant Nutr. 25:169-176.

Moraghan, J.T., J. Padilla, J.D. Etchevers, K. Grafton, and J.A. Acosta-Gallegos. 2002. Iron accumulation in seed of common bean. Plant Soil 246:175-183.

Morgounov, A., H.F. Gomez-Becerra, A. Abugalieva, M. Dzhunusova, M. Yessimbekova, H. Muminjanov, Y. Zelenskiy, L. Ozturk, and I. Cakmak. 2007. Iron and zinc density in common wheat grown in Central Asia. Euphytica 155:193-203.

Mori, S. 1999. Iron acquisition by plants. Curr. Opin. Plant Biol. 2:250-253.

Morris, C.E., and D.C. Sands. 2006. The breeder's dilemma -Yield or nutrition?. Nat. Biotechnol. 24:1078-1080.

Murphy, K.M., L.A. Hoagland, P.G. Reeves, B.-K. Baik, and S.S. Jones. 2009. Nutritional and quality characteristics expressed in 31 perennial wheat breeding lines. Renewable Agric. Food Systems 24:285-292.

Murphy, K.M., P.G. Reeves, and S.S. Jones. 2008. Relationship between yield and mineral nutrient concentrations in historical and modern spring wheat cultivars. Euphytica 163:381-390.

Murray, C.J.L., and A.D. Lopez (eds.). 1996. Accounting Principles Fifth Canadian Edition Vol 1 The global burden of disease. Vols. I and II. Harvard University Press, Cambridge, MA.

Nair, K.M. and V. Iyengar. 2009. Iron content, bioavailability and factors affecting iron status of Indians. Indian J. Med. Res. 130:634-645.

Naqvi, S., G. Farré, G. Sanahuja, T. Capell, C. Zhu, and P. Christou. 2009a. When more is better: Multigene engineering in plants. Trends Plant Sci. 15:48-56.

Naqvi, S., C. Zhu, G. Farre, K. Ramessar, L. Bassie, J. Breitenbach, G.P. Conesa, G. Ros, G. Sandmann, T. Capell, and P. Christou. 2009b. Transgenic multivitamin corn through biofortification of endosperm with three vitamins representing three distinct metabolic pathways. Proc. Natl. Acad. Sci. USA 106:7762-7767.

Neelam, K., N. Rawat, V.K. Tiwari, S. Kumar, P. Chhuneja, K. Singh, G.S. Randhawa, and H.S. Dhaliwal. 2010a. Introgression of group 4 and 7 chromosomes of Ae. peregrina in wheat enhances grain iron and zinc density. Mol. Breed. 28:623-634.

Neelam, K., V.K. Tiwari, N. Rawat, S.K. Tripathi, G.S. Randhawa, and H.S. Dhaliwal. 2010b. Identification of Aegilops species with higher production of phytosiderophore and iron and zinc uptake under micronutrient-sufficient and -deficient conditions. Plant Genet. Resour. 8:132-141.

Nestel, P., H.E. Bouis, J.V. Meenakshi, and W. Pfeiffer. 2006. Biofortification of staple food crops. J. Nutr. 136:1064-1067. 
Neumann, G., and V. Römheld. 2002. Root-induced changes in the availability of nutrients in the rhizosphere. p. 617-649. In: Y. Waisel, A. Eshel, and U. Kafkafi (eds.), Accounting Principles Fifth Canadian Edition Vol 1 Plant roots: The hidden half. Marcel Dekker, New York.

Nowack, B., I. Schwyzer, and R. Schulin. 2008. Uptake of Zn and Fe by wheat (Triticum aestivum var. Greina) and transfer to the grains in the presence of chelating agents (ethylenediaminedisccinic acid and ethylenediaminetetraacetic acid). J. Agric. Food Chem. 56:4643-4649.

Nozoye, T., S. Nagasaka, T. Kobayashi, M. Takahashi, Y. Sato, N. Uozumi, H. Nakanishi, and N.K. Nishizawa. 2011. Phytosierophore efflux transporters are crucial for iron acquision in graminaceous plants. J. Biol. Chem. 286:5446-5454.

Oatway, L., T. Vasanthan, and J. Helm. 2001. Phytic acid. Food Rev. Int. 17:417-431.

Ockenden, I., J.A. Dorsch, M.M. Reid, L. Lin, L.K. Grant, V. Raboy, and J.N.A. Lott. 2004. Characterization of the storage of phosphorus, inositol phosphate and cations in grain tissues of four barley (Hordeum vulgare L.) low phytic acid genotypes. Plant Sci. 167:1131-1142.

Oikeh, S.O., A. Menkir, B. Maziya-Dixon, R.M. Welch, and R.P. Glahn. 2003a. Genotypic differences in concentration and bioavailability of kernel-iron in tropical maize varieties grown under field conditions. J. Plant Nutr. 26:2307-2319.

Oikeh, S.O., A. Menkir, B. Maziya-Dixon, R. Welch, and R.P. Glahn. 2003b. Assessment of concentrations of iron and zinc and bioavailable iron in grains of early-maturing tropical maize varieties. J. Agric. Food Chem. 51:3688-3694.

Oikeh, S.O., A. Menkir, B. Maziya-Dixon, R. Welch, and R.P. Glahn. 2004a. Assessment of iron bioavailability from twenty elite late-maturing tropical maize varieties using an in vitro digestion/Caco-2 cell model. J. Sci. Food Agric. 84:1202-1206.

Oikeh, S.O., A. Menkir, B. Maziya-Dixon, R. Welch, R.P. Glahn, and G. Gauch Jr., 2004b. Environmental stability of iron and zinc concentrations in grain of elite early-maturing tropical maize genotypes grown under field conditions. J. Agric. Sci. 142:543-551.

Oliver, R.E., C. Yang, G. Hu, V. Raboy, and M. Zhang. 2009. Identification of PCR-based DNA markers flanking three low phytic acid mutant loci in barley. J, Plant Breed. Crop Sci. 14:87-93.

Oltmans, S.E., W.R. Fehr, G.A. Welke, and S.R. Cianzio. 2004. Inheritance of low-phytate phosphorus in soybean. Crop Sci. 44:433-435.

Oltmans, S.E., W.R. Fehr, G.E. Welke, V. Raboy, and K.L. Petersen. 2005. Agronomic and seed traits of soybean lines with low phytate. Crop Sci. 45:593-598.

Oomah, B.D., C. Blanchard, and P. Balasubramanian. 2008. Phytic acid, phytase, minerals, and antioxidant activity in Canadian dry bean (Phaseolus vulgaris L.) cultivars. J. Agric. Food Chem. 56:11312-11319.

Ortiz-Monasterio, J.I., N. Palacios-Rojas, E. Meng, K. Pixley, R. Trethowan, and R.J. Peña. 2007. Enhancing the mineral and vitamin content of wheat and maize through plant breeding. J. Cereal Sci. 46:293-307.

O'Sullivan, L., M.A. Jiwan, T. Daly, N.M. O’Brien, and S.A. Aherne. 2010. Bioaccessibility, uptake, and transport of carotenoids from peppers (Capsicum spp.) using the coupled in vitro digestion and human intestinal Caco-2 cell model. J. Agric. Food Chem, 58:53745379.

Ozturk, L., M.A. Yazici, C. Yucel, A. Torun, C. Cekic, A. Bagci, H. Ozkan, H.-J! Braun, Z. Sayers, and I. Cakmak. 2006. Concentration and localization of zinc during seed development and germination in wheat. Physiol. Plant 128:144-152. 
Pachón, H., D.A. Ortiz, C. Araujo, M.W. Blair, and J. Restrepo. 2009. Iron, zinc and protein bioavailability proxy measures of meals prepared with nutritionally enhanced beans. J. Food Sci. 74:H147-H154.

Paine, J.A., C.A. Shipton, S. Chaggar, R.M. Howells, M.J. Kennedy, G. Vernon, S.Y. Wright, E. Hinchliffe, J.L. Adams, A.L. Silverstone, and R. Drake. 2005. Improving the nutritional value of golden rice through increased pro-vitamin A content. Nat. Biotechnol. 23:482-487.

Paredes, M., V. Becerra, and J.U. Tay. 2009. Inorganic nutritional composition of common bean (Phaseolus vulgaris L.) genotypes race Chile. Chilean J. Agric. Res. 69:486-495.

Paterson, A.H., J.E. Bowers, R. Bruggmann, I. Dubchak, J. Grimwood, H. Gundlach, G. Haberer; U. Hellsten et al. 2009. The Sorghum bicolor genome and the diversification of grasses. Nature 457:551-556.

Patterson; J.K., X.G. Lei, and D.D. Miller: 2008. The pig as an experimental model for elucidating the mechanisms governing dietary influence on mineral absorption. Expt. Bioli Med. 233:651-664.

Pearson, J.N., and Z. Rengel. 1994. Distribution and remobilization of Zn and Mn duxing grain development in wheat. J. Expt, Bot, 45:1829-1835.

Pearson, J.N., and Z. Rengel. 1995. Uptake and distribution of ${ }^{65} \mathrm{Zn}$ and ${ }^{54} \mathrm{Mn}$ in wheat grown at sufficient and deficient levels of $\mathrm{Zn}$ and $\mathrm{Mn}$. II. During grain development. J. Expt. Bot. 46:841-845.

Peleg, Z., I. Cakmak, L. Ozturk, A. Yazici, Y. Jun, H. Budak, A.B. Korol, T. Fahima, and Y. Saranga. 2009. Quantitative trait loci conferring grain mineral nutrient concentrations in durum wheat $\times$ wild emmer wheat RIL population. Theor. Appl. Genet. 119:353-369.

Peremarti, A., R.M. Twyman, S. Gómez-Galeria, S. Naqvi, G. Farré, M. Sabalza, B. Miralpeix, S. Dashevskaya, D. Yuan, K. Ramessar, P. Christou, C. Zhu, L. Bassie, and T. Capell, 2010. Promoter diversity in multigene transformation. Plant Mol. Biol. 73:363-378.

Persson, D.P., T.H. Hansen, K.H. Laursen, J.K. Schjoerring, and S. Husted 2009. Simultaneous iron, zinc, sulfur and phosphorus speciation analysis of barley grain tissues using SEC-ICP-MS and IP-ICP-MS. Metallomics 1:418-426.

Petry, N., I. Egli, C. Zeder, T. Walczyk, and R. Hurrell. 2010. Polyphenols and phytic acid contribute to the low iron bioavailability from common beans in young women. J. Nutr. 140:1977-1982.

Pfeiffer, W.H., and B. McClafferty. 2007. HarvestPlus: Breeding crops for better nutrition. Crop Sci. 47(S3):S88-S105.

Phan-Thien, K.-Y., G.C. Wright, and N. A. Lee. 2010. Genotype-by-environment interaction affects the essential mineral composition of peanut (Arachis hypogaea L.) kernels. J. Agric. Food Chem. 58:9204-9213.

Pilu, R., D. Panzeri, G. Gavazzi, S.K. Rasmussen, G. Consonni, and E. Nielsen. 2003. Phenotypic, genetic and molecular characterization of a maize low phytic acid mutant (Ipa 241). Theor. Appl. Genet. 107:980-987.

Pinheiro, C., J.P. Baeta, A.M. Pereira, H. Domingues, and C.P. Ricardo. 2010. Diversity of seed mineral composition of Phaseolus vulgaris L. germplasm. J. Food Comp. Anal. 23:319-325.

Pirzadah, M., M. Afyuni, A. Khoshgoftarmanesh, and R. Schulin. 2010. Micronutrient status of calcareous soils and rice products: Implication for human health. Biol. Fertil. Soils 46:317-322.

Pixley, K.V., N. Palacios-Rojas, and R.P. Glahn, 2011a. The usefulness of iron bio-availability as a target trait for breeding maize (Zea mays L.) with enhanced nutritional value. Field Crops Res. 123:153-160. 
Pixley, K., R. Babu, J. Yan, and N. Palacios, 2011b. Challenges, progress, and state of breeding for provitamin A. Symposia Brief: Progress, Challenges, \& the Way Forward in Breeding \& Gene Discovery: Vitamin A. First Global Conference on Biofortification. November 9-11, 2010. Washington, DC.

Platel, K., S.W. Eipeson, and K. Srinivasan. 2010. Bioaccessible mineral content of malted finger millet (Eleusine coracana), wheat (Triticum aestivum), and barley (Hordeum valgare). J. Agric. Food Chem. 58:8100-8103.

Pleijel, H., and H. Danielsson. 2009. Yield dilution of grain $\mathrm{Zn}$ in wheat in open-top chamber experiments with elevated $\mathrm{CO}_{2}$ and $\mathrm{O}_{3}$ exposure. J. Cereal Crops 50:278282.

Ponnamperuma, F.N. 1972. The chemistry of submerged soils. Adv. Agron. 24:29-96.

Prasad, M.N.V., and N. Nirupa, 2007. Phytoferritins-Implications for human health and nutrition. Asian Aust. J. Plant Sci. Biotechnol. (http://www.globalsciencebooks.inf/ JournalsSup/Images/SF/AAJPSB_1.(1)1-9.pdf).

Pray, C., R. Paarlberg, and L. Unnevehr, 2007. Patterns of political response to biofortified varieties of crops produced with different breeding techniques and agronomic traits. AgBioForum 10:135-143.

Qaderi, M.M., and D.M. Reid. 2009. Crop responses to elevated carbon dioxide and temperature. p. 1-19. In: S.N. Singh (ed.), Accounting Principles Fifth Canadian Edition Vol 1 Climate change and crops. Springer-Verlag, Berlin.

Qaim, M., A.J. Stein, and J.V. Meenakshi. 2007. Economics of biofortification. HarvestPlus, International Food Policy Research, Washington, DC (http://ideas.repec.org/a/bla/ agecon/v37y2007is/1p119-133.html).

Qu, L.Q., T. Yoshihara, A. Ooyama, F, Goto, and F. Takaiwa. 2005. Iron accumulation does not parallel the high expression level of ferritin in transgenic rice seeds. Planta 222:225-233.

Quackenbush, F.W., J.G. Firch, A.M. Brunson, and L.R. House. 1963. Carotenoid, oil, and tocopherol content of corn inbreds. Cereal Chem. 40:250-259.

Raboy, V. 1997. Accumulation and storage of phosphates and minerals. p.441-447. In: B.A. Larkins and I.K. Vasil (eds.), Accounting Principles Fifth Canadian Edition Vol 1 Advances in cellular and molecular biology of plants. Vol. 4: Cellular and molecular biology of plant seed development. Kluwer Academic Publ,, Dordrecht, The Netherlands.

Raboy, V. 2000. Low-phytic acid grains. Food Nutr. Bull. 21:423-427.

Raboy, V. 2001. Seeds for a better future: Low phytate grains help to overcome malnutrition and reduce pollution. Trends Plant Sci. 6:458-462.

Raboy, V. 2002. Progress in breeding for low phytate crops. J. Nutr. 132:503S-505S.

Raboy, V., M.M. Noaman, G.A. Taylor, and S.G. Pickett. 1991. Grain phytic acid and protein are highly correlated in winter wheat. Crop Sci. 31:631-635.

Raboy, V., K.A. Young, J.A. Dorsch, and A. Cook. 2001. Genetics and breeding of seed phosphorus and phytic acid. J. Plant Physiol, 158:489-497.

Rai, K.N., K.A. Kumar, D.J. Andrews, A.S. Rao, A.G.B. Raj, and J.R. Witcombe. 1990. Registration of ICTP 8203 pearl millet. Crop Sci. 30:959.

Ramaswami, B. 2007. Biofortified crops and biotechnology: A political economy landscape for India. AgBioForum 10:170-177.

Ramachandran, A., C.J. Pozniak, J.M. Clarke, and A.K. Singh. 2010. Carotenoid accumulation during grain development in durum wheat. J. Cereal Sci. 52:30-38.

Ramessar, K., T. Capell, R.M. Twyman, H. Quemada, and P. Christou. 2008. Trace and traceability-A call for regulatory harmony. Nat. Biotechnol. 26:975-978. 
Ramessar, K., T. Capell, R.M. Twyman, H. Quemada, and P. Christou, 2009. Calling the tunes on transgenic crops: The case for regulatory harmony. Mol. Breed. 23:99-112.

Rao, D.E.C.S., K.V. Rao, T.P. Reddy, and V.D. Reddy. 2009. Molecular characterization, physicochemical properties, known and potential applications of phytases: An overview. Crit. Rev. Biotechmol. 29:182-198.

Ravet, K., B. Touraine, S.A. Kim, F. Cellier, S. Thomine, M.L. Guerinot, J.-F, Briat, and F. Gaymard, 2009. Post-transregulation of AtFER2 ferritin in response to intracellular iron trafficking during fruit development in Arabidopsis. Mol. Plant 2:1095-1106.

Rawat, N., V.K. Tiwari, K. Neelam, G.S. Randhawa, P. Chhuneja, K. Singh, and H.S. Dhaliwal. 2009a. Development and characterization of Triticum aestivum-Aegilops kotschyi amphiploids with high grain iron and zinc contents. Plant Genet. Resour. 7:271-280.

Rawat, N., V.K. Tiwari, N. Singh, G.S. Randhawa, K. Singh, P. Chhuneja, and H.S. Dhaliwal. 2009b. Evaluation and utilization of Aegilops and wild Triticum species for enhancing iron and zinc content in wheat. Genet. Resour. Crop Evol. 56:53-64.

Raybould, A., A. Tuttle, S. Shore, and T. Stone. 2010. Environmental risk assessments for transgenic crops producing output trait enzymes. Transgenic Res. 19:595-609.

Reboul, E., M. Richelle, E. Perrot, C. Desmoulins-Malezet, V. Pirisi, and P. Borel. 2006. Bioaccessibility of carotenoids and vitamin E from their main dietary sources. J. Food Agric. Chem. 54:8749-8755.

Reddy, M.B., and J.D. Cook. 1991. Assessment of dietary determinants of nonheme-iron absorption in humans and rats. Am. J. Clin. Nutr, 54:723-728.

Reddy, B.V.S., S. Ramesh, and T. Longvah. 2005. Prospects of breeding for micronutrients and $\dot{\beta}$-carotene-dense sorghums. Int. Sorghum Millet Newslett. 46:10-14.

Rego, T.J., K.L. Sahrawat, S.P. Wani, and G. Pardhasaradhi. 2007. Widespread deficiencies of sulfur, boron, and zinc in Indian semi-arid tropical soils: On-farm crop responses. J. Plant Nutr. 30:1569-1583.

Rengel, R. 2001, Genotypic differences in micronutrient use efficiency in crops. Commun. Soil Sci. Plant Anal. 32:1163-1186.

Rengel, Z. 2002. Genetic control of root exudation. Plant Soil 245:59-70.

Rengel, Z., and R.D. Graham. 1995. Importance of seed zinc content for wheat growth on zinc deficient soils. I. Vegetative growth. Plant Soil 173:259-266.

Rengel, Z., and V. Römheld. 2000. Root exudation and Fe uptake and transport in wheat genotypes differing in tolerance to Zn deficiency. Plant Soil 222:25-34.

Riedel, W.E. 2010. Mineral-nutrient synergism and dilution responses to nitrogen fertilizer in field-grown maize. J. Plant Nutr. Soil Soc. 173:869-874.

Rodriguez-Amaya, D.B., and M. Kimura. 2004. Accounting Principles Fifth Canadian Edition Vol 1 HarvestPIus handbook for carotenoid analysis. HarvestPlus Technical Monograph 2. International Food Policy Research Institute (IFPRI); International Center for Tropical Agriculture (CIAT), Washington, DC; Cali.

Römheld, V., and H. Marschner. 1986. Evidence for a specific uptake system for iron phytosiderophore in roots of grasses. Plant Physiol. 80:175-180.

Ronald, P. 2011. Plant genetics, sustainable agriculture and global food security. Genetics 188:11-20.

Rosado, J.L., K.M. Hambidge, L.V. Miller, O.P. Garcia, J. Westcott, K. Gonzalez, J. Conde, C. Hotz, W. Pfeiffer, I. Ortiz-Monasterio, and N.F. Krebs. 2009. The quantity of zinc absorbed from wheat in adult women is enhanced by biofortification. J. Nutr. 139:1920-1925.

Rubey, L., R.W. Ward, and D. Tschirley. 1997. Maize research priorities. The role of consumer preferences. p. 145-155. In: D. Byerlee, and C.K. Eicher (eds.), Accounting 
Principles Fifth Canadian Edition Vol 1 Africa's emerging maize revolution. Lynne Rienner Publishers, Boulder, Colorado, USA.

Rutger, J.N., V. Raboy, K.A.K. Moldenhauer, R.J. Bryant, F.N. Lee, and J.W. Gibbons. 2004. Registration of KBNT lpa1-1 low phytic acid germplasm of rice. Crop Sci. 44:363.

Ryan, M.H., and J.H. Graham. 2002. Is there a role for arbuscular mycorrhizal fungi in production agriculture. Plant Soil 244:263-271.

Ryan, C.G., D.P. Siddons, R. Kirkham, P.A. Dunn, A. Kuczewski, G. Moorhead, G. de Geronimo, D.J. Paterson, M.D. de Jonge, R.M. Hough, M.J. Lintern, D.L. Howard, P. Kappen, and J. Cleverley. 2010. The new Maja detector system: Methods for high definition trace element imaging of natural material. AIP Conf. Proc. 1221:9-17.

Sachs, J.D., and J.W. McArthur. 2005. The millennium project: A plan for meeting the millennium development goals. Lancet 365:347-353.

Sadeghzadeh, B., Z. Rengel, C. Li, and H. Yang. 2010. Molecular marker linked to a chromosome region regulating seed $\mathrm{Zn}$ accumulation in barley. Mol. Breed. 25:167-177.

Saghai-Maroof, M.A., N.M. Glover, R.M. Biyashev, G.R. Buss, and E.A. Grabau. 2009. Genetic basis of the low-phytate trait in the soybean line CX1834. Crop Sci. 49:69-76.

Sahrawat, K.L. 2000. Macro- and micro-nutrients removed by upland and lowland rice cultivars in West Africa. Commun. Soil Sci. Plant Anal. 31:717-723.

Sahrawat, K.L. 2007. Soil fertility advantages of submerged rice cropping systems: A review. J. Sustain. Agric. 31:5-23.

Sahrawat, K.L. 2009. The role of tolerant genotypes and plant mutrients in reducing acidsoil infertility in upland rice: An appraisal. Arch. Agron. Soil Sci. 55:597-607.

Sahrawat, K.J., G.R. Kumar, and K.V.S. Murthy. 2002. Evaluation of triacid and dry ashing procedures for determining potassium, calcium, magnesium, iron, zinc, manganese, and copper in plant materials. Commun. Soil Sci. Plant Anal. 33:95-102.

Sahrawat, K.L., T.J. Rego, S.P. Wani, and G. Pardhasaradhi. 2008. Sulfur, boron, and zino fertilization effects on grain and straw quality of maize and sorghum grown on farmers' fields in the semi-arid tropical region of India. J. Plant Nutr. 31:1578-1584.

Salas Fernandez, M.G., M.T. Hamblin, L. Li, W.L. Rooney, M.L. Tuinstra, and S. Kresovich. 2008. Quantitative trait loci analysis of endosperm color and carotenoids content in sorghum grain. Crop Sci. 48:1732-1743.

Salas Fernandez, M.G., I. Kapran, S. Souley, M. Abdou, I.H. Maiga, C.B. Acharya, M.T. Hamblin, and S. Kresovich. 2009. Collection and characterization of yellow endosperm sorghums from West Africa for biofortification. Genet. Resour. Crop Evol. 56:991-1000.

Salunke, R., K. Neelam, N. Rawat, V.K. Tiwari, H.S. Dhaliwal, and P. Roy. 2011. Bioavailability of iron from wheat Aegilops derivatives selected for high grain iron and protein contents. J. Agric. Food Chem. 59:7465-7473.

Santra, M., D.K. Santra, V.S. Rao, S.P. Taware, and S.A. Tamhankar, 2005. Inheritance of B-carotene concentration in durum wheat (Triticum turgidum L. ssp. durum). Euphytica 144:215-221.

Sawada, Y., K. Akiyama, A. Sakata, A. Kuwahara, H. Otsuki, T. Sakurai, K. Saito, and M.Y. Hirai. 2009. Widely targeted metabolomics based on large-scale MS/MS data for elucidating metabolite accumulation patterns in plants. Plant Cell Physiol. 50:37-47.

Saxena, D., and G. Stotzky. 2001. Bt corn has a higher lignin content than non-Bt corn. Am. J. Bot. 88:1704-1706.

Schanable, P.S., D. Ware, R.S. Fulton, J.C. Stein, F. Wei, S. Pasternak, P. Minx, A.D. Reily, L. Courtney, S.S. Kruchowski et al. 2009. The B73 maize genome: Complexity, diversity, and dynamics. Science 326:1112-1115. 
Scheckel, K.G., R. Hamon, L. Jassogne, M. Rivers, and E. Lombi. 2007. Synchrotron X-ray absorption-edge computed microtomography imaging of thallium compartmentalization in Iberis intermedia. Plant Soil 290:51-60.

Schlenker, W., and M.J. Roberts. 2009. Nonlinear temperature effects indicate severe damages to US crop yields under climate change. Proc. Natl. Acad. Sci. USA 106:1559415598.

Schmutz, J., S.B. Cannon, J. Schluter, J. Ma, T. Mitros, W. Nelson, D.L. Hyten et al. 2010. Genome sequence of the palaeopolyploid soybean. Nature 463:178-183.

Schurr, U. 1999. Dynamics of nutrient transport from the root to the shoot. Prog. Bot. 60:234-253.

Scott, M.P., J.W. Edwards, C.P. Well, J.R. Schussler, and J.S. Smith. 2006. Grain composition and amino acid content in maize cultivars representing 80 years of commercial maize varieties. Maydica 51:417-423.

Sellappan, K., K. Datta, V. Parkhi, and S.K. Datta. 2009. Rice caryopsis structure in relation to distribution of micronutrients (iron, zinc, $\beta$-carotene) of rice cultivars including transgenic indica rice. Plant Sci. 177:557-562.

Selvi, M.G.B., and S. Rajarathinam. 2009. Correlation studies for grain yield components and nutritional quality in pearl millet (Pennisetum glaucum (L.) R. BR.) germplasm. World J. Agric. Sci. 5:686-689.

Sharma, K.D., B.M. Singh, and R.S. Chauhan. 1995. An improved medium for in vitro grain development from detached wheat spikelets. Plant Cell Tissue Organ Cult. 42:227-231.

Shi, R., H. Li, Y. Tong, R. Jing, F. Zhang, and C. Zou. 2008. Identification of quantitative trait locus of zinc and phosphorus density in wheat (Triticum aestivum L.) grain. Plant Soil 306:95-104.

Shi, J.R., H.Y. Wang, K. Schelin, B. Li, M. Faller, J.M. Stoop, R.B. Meeley, D.S. Ertl, J.P. Ranch, and K. Glassman. 2007. Embryo-specific silencing of a transporter reduces phytic acid content of maize and soybean seeds. Nat. Biotechnol. 25:930-937.

Šimic, D., R. Sudar, T. Ledenčan, A. Jambrovic, Z. Zdunic, I. Brkić, and V. Kovačević. 2009. Genetic variation of bioavailable iron and zinc in grain of a maize population. J. Cereal Sci. 50:392-397.

Singh, M.V. 2008. Micronutrient deficiencies in crops and soils in India. p. 93-125. In: B.J. Alloway (ed.), Accounting Principles Fifth Canadian Edition Vol 1 Micronutrient deficiencies in global crop production. Springer, Berlin.

Singh, K., P. Chhuneja, V.K. Tiwari, N. Rawat, K. Neelam, R. Aggarwal, S. Malik, B. Keller, and H.S. Dhaliwal. 2010a. Mapping of QTL for grain iron and zinc content in diploid A genome wheat and validation of these loci in $U$ and $S$ genomes. p. 304, Plant and Animal Genomes XVIII Conference. San Diego, CA, USA.

Singh, S., S. Mishra, and K. Kalpana. 2010b. Phenotypic diversity in grain yield and nutritional traits of rice (Oryza sativa L.). Int. Rice Res. Notes 35:1-4

Singh, B.K., and C.F. Jenner. 1983. Culture of detached ears of wheat in liquid culture: Modification and extension of the method. Aust. J. Plant Physiol. 10:227-236.

Sinha, P.K., R. Kapoor, D.C. Uprety, and A.K. Bhatnagar. 2009. Impact of elevated $\mathrm{CO}_{2}$ concentration on ultrastructure of pericarp and composition of grain in three Triticum species of different ploidy levels. Environ. Expt. Bot. 66:451-456.

Sison, M.E.G.Q., G.B. Gregorio, and M. Mendiore. 2006. The effect of different milling times on grain iron content and grain physical parameters associated with milling of eight genotypes of rice (Oryza sativa L.). Philippine J. Sci. 135:9-17.

Spear; J.D., and W.R. Fehr. 2007. Genetic improvement of seedling emergence of soybean lines with low phytate. Crop Sci. 47:1354-1360. 
Sperotto, R.A., T. Boff, G.L. Duarte, L.S. Santos, M.A. Grusak, and J.P. Fett. 2010. Identification of putative target genes to mamipulate $\mathrm{Fe}$ and $\mathrm{Zn}$ concentrations in rice grains. J. Plant Physiol. 167:1500-1506.

Ssemakula, G., and W. Pfeiffer. 2011. Considerations for implementation of biofortification in developing countries: The case of Sub-Saharan Africa. Symposia Brief: Progress, Challenges, and the Way Forward in Breeding and Gene Discovery: Vitamin A. First Global Conference on Biofortification. November 9-11, Washington, DC.

Stangoulis, J.C.R., B.-L. Huynh, R.W. Welch, E.-Y. Choi, and R.D. Graham. 2007. Quantitative trait loci for phytate in rice grain and their relationship with grain micronutrient content. Euphytica 154:289-294.

Stangoulis J., and C. Sison (eds.). 2008. Accounting Principles Fifth Canadian Edition Vol 1 Crop sampling protocols for micronutrient analysis. HarvestPlus Technical Monograph 7. International Food Policy Research Institute (IFPRI); International Center for Tropical Agriculture (CIAT), Washington, DC; Cali.

St. Clair, S.B., and J.P. Lynch. 2010. The opening of pandora's box: Climate change impacts on soil fertility and crop nutrition in developing countries. Plant Soil 335:101-115.

Stein, A.J. 2006. Accounting Principles Fifth Canadian Edition Vol 1 Micronutrient malnutrition and the impact of modern plant breeding on public health in India: How cost-effective is biofortification? Cuvillier Verlag, Göttingen.

Stein, A.J. 2010. Global impacts of human mineral nutrition. Plant Soil 335:133-154.

Stein, A.J., J.V. Meenakshi, M. Qaim, P. Nestel, H.P.S. Sachdev, and Z.A. Bhutta. 2008.

Potential impact of iron biofortification in India. Social Sci. Med. 66:1797-1808.

Stein, A.J., P. Nestel, J.V. Meenakshi, M. Qaim, H.P.S. Sachdev, and Z.A. Bhutta. 2007.

Plant breeding to control zinc deficiency in India: How cost-effective is biofortification?

Public Health Nutr. 10:492-501.

Steinke, K., P. Guertler, V. Paul, S. Wiedemann, T. Ettle, C. Albrecht, H.H.D. Meyer, and H.

Spiekers. 2010. Effects of long-term feeding of genetically modified corn (event MON810) on the performance of lactating dairy cows. J. Anim. Physiol. Anim. Nutr. 94:e185-e193.

Stevens, R., and A. Winter-Nelson. 2008. Consumer acceptance of provitamin A-biofortified maize in Maputo, Mozambique. Food Policy 33:341-351.

Stewart, W.M., D.W. Dibb, A.E. Johnston, and T.J. Smyth. 2005. The contribution of commercial fertilizer nutrients to food production. Agron. J. 97:1-6.

Stomph, T.J., W. Jiang, and P.C. Struik. 2009. Zinc biofortification of cereals: Rice differs from wheat and barley. Trends Plant Sci. 14:123-124.

Street, K., M. Mackay, E. Zuev, N. Kaul, M. El Bouhssini, J. Konopka, and O. Mitrofanova. 2008. Swimming in the genepool: A rational approach to exploiting large genetic resource collections. p. 1-4. In: R. Appels, R. Eastwood, E. Laguddah, P. Langridge, M. Mackay, L. McIntyre, and P. Sharp (eds.), 11th International Wheat Genetics Symposium. Sydney University Press, Sydney, Australia (http://hd1.handle.net/2123/3390).

Sturkie, P. 2000. Accounting Principles Fifth Canadian Edition Vol 1 Avian physiology. Academic Press, San Diego, CA.

Suharno. D., C.E. West, Muhilal, D. Karyadi, and J.G. Hautvast. 1993. Supplementation with vitamin $A$ and iron for nutritional anaemia in pregnant women in West Java, Indonesia. Lancet 27:1325-1328.

Sunde, R.A. 2010. mRNA transcripts as molecular biomarkers in medicine and nutrition. J. Nutr. Biochem. 21:665-670.

Takagi, S. 1976. Naturally occurring iron-chelating compounds in oat- and rice-root washings. Soil Sci. Plant Nutr. 22:423-433. 
Takahashi, M., H. Nakanishi, S. Kawasaki, N.K. Nishizawa, and S. Mori. 2001. Enhanced tolerance of rice to low iron availability in alkaline soils using barley nicotianamine aminotransferase genes, Nat. Biotechnol. 19:466-469.

Takahashi, M., T. Nozoye, N. Kitajima, N. Fukuda, A. Hokura, Y. Terada, I. Nakai, Y. Ishimaru, T. Kobayashi, H. Nakanishi, and NiK. Nishizawa. 2009. In vivo analysis of metal distribution and expression of metal transporters in rice seed during germination process by microarray and X-ray fluorescence imaging of $\mathrm{Fe}, \mathrm{Zn}, \mathrm{Mn}$, and $\mathrm{Cu}$. Plant Soil 325:39-51.

Tako, E., R.P. Glahn, J.M. Laparra, R.M. Welch, X. Lei, J.D. Kelly, M.A. Rutzke, and D.D. Miller. 2009a. Iron and zinc bioavailabilities to pigs from red and white beans (Phaseolus vulgaris L.) are similar. J. Agric. Food Chem. 57:3134-3140.

Tako, E., M.A. Rutzke, and R.P. Glahn. 2009b. Using the domestic chicken (Gallus gallus) as an in vivo model for iron bioavailability. Poult. Sci. 89:514-521.

Talukder, Z.I., E. Anderson, P.N. Miklas, M.W. Blair, J. Osorno, M. Dilwari, and K.G. Hossain. 2010. Genetic diversity and selection of genotypes to enhance $\mathrm{Zn}$ and $\mathrm{Fe}$ content in common bean. Can. J. Plant Sci. 90:49-60.

Tan, J., N. Baisakh, N. Oliva, V. Parkhi, M. Rai, L. Torrizo, K. Datta, and S.K. Datta. 2005. The screening of rice germplasm, including those of transgenic rice lines which accumulate $\beta$-carotene in their polished seeds, for their carotenoids profile. Int. J. Food Sci. Technol. 40:563-569.

Tan, S.Y., C.K. Yeung, E. Tako, R.P. Glahn, R.M. Welch, X. Lei, and D.D. Miller. 2008. Iron bioavailability to piglets from red and white common beans (Phaseolus vulgaris). J. Agric. Food Chem. 56:5008-5014.

Tang, G: 2010. Bioconversion of dietary provitamin A carotenoids to vitamin A in humans. Am. J. Clin. Nutr. 91(Suppl.):1468S-1473S.

Tang, G., J. Qin, G.G. Dolnikowski, R.M. Russell, and M.A. Grusak, 2009. Golden rice is an effective source of vitamin A. Am. J. Clin. Nutr, 89:1776-1783.

Tanumihardjo, S.A. 2002. Factors influencing the conyersion of carotenoids to retinol: Bioavailability to bioconversion to bioefficacy. Int. J. Vitam. Nutr. Res. 72:40-45.

Taub, D.R., B. Miller, and H. Allen, 2008. Effects of elevated $\mathrm{CO}_{2}$ on the protein concentration of food crops: A meta analysis. Global Change Biol, 14:565-575.

Taub, D.R., and X. Wang. 2008. Why are nitrogen concentrations in plant tissues lower under elevated $\mathrm{CO}_{2}$ ? A critical examination of the hypotheses. J. Integr. Plant Biol. 50:1365-1374.

Tester, M, and P. Langridge. 2010. Breeding technologies to increase crop production in a changing world. Science 327:818-822.

Thakkar, S.K., and M.L. Failla. 2008. Bioaccessibility of pro-vitamin A carotenoids is minimally affected by non pro-vitamin A xanthophylls in maize (Zea mays sp.). J. Agric. Food Chem. 56:11441-11446.

Thakkar; S.K., T. Huo, B. Maziya-Dixon, and M.L. Failla. 2009. Impact of style of processing on retention and bioaccessibility of $\beta$-carotene in cassava (Manihot esculenta, Crantz). J. Agric. Food Chem. 57:1344-1348.

Thavarajah, P., D. Thavarajah, and A. Vandenberg. 2010. Low phytic; acid lentils (Lens culinaris L.): A potential solution for. increased micronutrient bioavailability. J. Agric. Food Chem. 57:9044-9049.

Theil, E.C. 1987. Ferritin: Structure, gene regulation, and cellular function in animals, plants, and microorganisms. Annu. Rev, Biochem. 56:289-315,

Thome, J.H. 1985. Phloem unloading of $\mathrm{C}$ and $\mathrm{N}$ assimilates in developing seeds. Ann. Rev. Plant Physiol, 36:317-343. 
Thurnham, D.I., L.D. McCabe, S. Haldar, F.T. Wieringa, C.A. Northrop-Clewes, and G.P. McCabe. 2010. Adjusting plasma ferritin concentrations to remove the effects of subclinical inflammation in the assessment of iron deficiency: A meta-analysis. Am. J. Clin. Nutr. 92:546-555.

Tisdale, S.L., and W.L. Nelson. 1975. Accounting Principles Fifth Canadian Edition Vol 1 Soil fertility and fertilizer, 3rd edn. Macmillian Publishing Co., Inc., USA.

Tiwari, V.K.; N. Rawat, P. Chhuneja, K. Neelam, R. Aggarawal, G.S. Randhawa, H.S. Dhaliwal, B. Keller, and K. Singh. 2009. Mapping of quantitative trait loci for grain iron and zinc concentration in diploid A genome wheat. J. Hered. 100:771-776.

Tiwari, V.K., N. Rawat, K. Neelam, S. Kumar, G.S. Randhawa, and H.S. Dhaliwal. 2010. Substitutions of $2 \mathrm{~S}$ and $7 \mathrm{U}$ chromosomes of Aegilops kotschy in wheat enhance grain iron and zinc concentrations. Theor. Appl. Genet. 121:259-269.

Tryphone, G.M., and S. Nchimbi-Msolla. 2010. Diversity of common bean (Phaseolus vulgaris L.) genotypes in iron and zinc contents under screenhouse conditions. Afr. J. Agric. Res. 5:738-747.

Tyssandier, V., B. Lyan, and P. Borel. 2001. Main factors goverming the transfer of carotenoids from emulsion lipid droplets to micelles. Biochim. Biophys. Acta 1533:285-292.

Uauy, C., A. Distelfeld, T. Fahima, A. Blechl, and J. Dubcovsky. 2006; A NAC gene regulating senescence improves grain protein, zinc, and iron content in wheat. Science 314:1298-1301.

Underwood, B.A. 1999. Accounting Principles Fifth Canadian Edition Vol 1 Micronutrient deficiencies as a public health problem in developing countries and effectiveness of supplementation, fortification, and nutrition education: Is there a role for agriculture? In: Improving human nutrition through agriculture: The role of international agricultural Research. International Food and Policy Research Institute (IFPRI), Washington, DC.

Upadhyaya, H.D. 2003. Phenotypic diversity in groundnut (Arachis hypogaea L.) core collection assessed by morphological and agronomical evaluations. Genet. Resour. Crop Evol. 50:539-550.

Upadhyaya, H.D. and R. Ortiz. 2001. A mini core collection for capturing diversity and promoting utilization of chickpea genetic resources in crop improvement. Theor. Appl. Genet. 102:1292-1298.

Upadhyaya, H.D., R. Ortiz, P.J. Bramel, and S. Singh. 2002. Phenotypic diversity for morphological and agronomic characteristics in chickpea core collection. Euphytica 123:333-342.

Upadhyaya, H.D., D. Yadav, K.N. Reddy, C.L.L. Gowda, and S. Singh. 2010. Development of pearl millet mini core collection for enhanced utilization of germplasm. Crop Sci. 51:217-223.

Vallabhaneni, R., C.E. Gallagher, N. Licciardello, A.J. Cuttriss, R.F. Quinlan, and E.T. Wurtzel, 2009. Metabolite sorting of a germplasm collection reveals the Hydroxylase3 locus as a new target for maize provitamin A biofortification, Plant Physiol. 151:16351645.

van den Berg, H. 1999. Carotenoid interactions. Nutr. Rev. 57:1-10.

van den Berg, H., and T. van Vilet. 1998. Effect of simultaneous single dose of $\beta$-carotene with lutein or lycopene on the $\beta$-carotene and retinyl ester responses in the triacylglycerol-rich lipo-protein fraction of men. Am. J. Clin. Nutr. 68:82-89.

van het Hof, K.H., C.E. West, J.A. Weststrate, and J.G.A.J. Hautvast. 1999. Dietary factors that affect the bioavailability of carotenoids. J. Nutr. 130:503-506. 
van Jaarsveld, P.J., M. Faber, S.A. Tanumihardjo, P. Nestel, C.J. Lombard, and A.J.S. Benadé. 2005. $\beta$-Carotene-rich orange-fleshed sweet potato improves the vitamin A status of primary school children assessed with the modified-relative-dose-response test. Am. J. Clin. Nutr. 81:1080-1087.

Varriano-Marston, E., and R.C. Hoseney. 1980. Note on mineral content and location in pearl millet. Cereal Chem. 57:150-152.

Varshney, R.K., S.N. Nayak, G.D. May, and S.A. Jackson. 2009. Next-generation sequencing technologies and their implications for crop genetics and breeding. Trends Biotechnol. 27:522-530.

Vasconcelos, M., K. Datta, N. Oliva, M. Khalekuzzaman, L. Torrizo, S. Krishnan, M. Oliveira, F. Goto, and S.K. Datta. 2003. Enhanced iron and zinc accumulation in transgenic rice with the ferritin gene. Plant Sci. 164:371-378.

Veda, S., K. Platel, and K. Srinivasan. 2008. Influence of food acidulants and antioxidant species on the bioaccessibility of $\beta$-carotene from selected vegetables. J. Agric. Food Chem. 56:8714-8719.

Velu, G., V.N. Kulkarni, V. Muralidharan, K.N. Rai, T. Longvah, K.L. Sahrawat, and T.S. Raveendran. 2006. A rapid screening method for grain iron content in pearl millet. SAT J. 2:4.

Velu, G., I. Ortiz-Monasterio, R.P. Singh, and T. Payne. 2011. Variation for grain micronutrient concentration in wheat core collection accessions of diverse origin. Asian $J$. Grop Sci. 3:43-48.

Velu, G, K.N. Rai, V. Muralidharàn, V.N. Kulkarni, T. Longvah, and T.S. Raveendran. 2007. Prospects of breeding biofortified pearl millet with high grain iron and zinc content. Plant Breed. 126:182-185.

Walker, D.R., A.M. Scaboo; V.R. Pantalone, J.R. Wilcox, and H.R. Boerma. 2006. Genetic mapping of loci associated with seed phytic acid content in CX1834-1-2 soybean. Crop Sci. 46:390-397.

Wang, Y.X., A. Specht, and W.J. Horst. 2011. Stable isotope labelling and zinc distribution in grains studied by laser ablation ICP-MS in an ear culture system reveals zinc transport barriers during grain filling. in wheat. New Phytol. 189:428-437.

Waters, B.M., U. Cristobal, J. Dubcovsky, and M.A. Grusak. 2009. Wheat (Triticum aestivum) NAM proteins regulate the translocation of iron, zinc, and nitrogen compounds from vegetative tissues to grain. J. Expt. Bot. 60:4263-4274.

Welch, R.M. 1986. Effects of nutrient densities on seed production and quality. Adv. Plant Nutr. 2:205-247.

Welch, R.M. 1995, Micronutrient nutrition of plants. Crit. Rev. Plant Sci. 14:49-82.

Welch, R.M., and R.D. Graham. 2002, Breeding crops for enhanced micronutrient content. Plant Soil 245:205-214.

Welch, R.M., and R.D. Graham. 2004. Breeding for micronutrients in staple food crops from a human perspective. J. Expt. Bot. 55:353-364.

Welch, R.W., and R.D. Graham. 2005. Agriculture: The real nexus for enhancing bioavailable micronutrients in food crops. J. Trace Elem. Med. Biol. 18:299-307.

Welch, R.M., W.A. House, S. Beebe, D. Senadhira, G.B. Grégorio, and Z. Cheng. 2000a. Testing iron and zinc bioavailability in genetically enríched beans ( $P$ haseolus vulgaris L.) and rice (Oryza sativa L.) in a rat model. Food Nutr, Bull. 21:428-433.

Welch, R.M., W.A. House, I. Ortiz-Monasterio, and Z. Cheng. 2000b. Potential for improving bioavailable zinc in wheat grain (Triticum species) through plant breeding. J. Agric. Food Chem. 53:2176-2180. 
Wessells, K.R., J.M. Jorgensen, S.Y. Hess, L.R. Woodhouse, J.M. Peerson, and K.H. Brown. 2010. Plasma zinc concentration responds to the initiation and discontinuation of shortterm zinc supplementation in healthy men. J. Nutr. 140:2128-2133.

Widodo, B., M.R. Broadley, T. Rose, M. Frei, J. Pariasca-Tanaka, T. Yoshihashi, M. Thomson, J.P. Hammond, A. Aprile, T.J. Close, A.M. Ismail, and M. Wissuwa. 2010. Response to zinc deficiency of two rice lines with contrasting tolerance is determined by root growth maintenance and organic acid exudation rates, and not by zinc-transporter activity. New Phytol. 186:400-414.

Wienecke, F., and J. Gruenwald. 2007. Nutritional supplementation: Is it necessary for everybody?. Adv. Ther. 24:1126-1135.

Wilcox, J.R., G.S. Premchandra, K.A. Young, and V. Raboy. 2000. Isolation of high seed inorganic P, low-phytate soybean mutants. Crop Sci. 40:1601-1605.

Wirth, J., S. Poletti, B. Aeschlimann, N. Yakandawala, B. Drosse, S. Osario, T. Tohge, A.S, Fernie, D. Günther, W. Gruissem, and C. Sautter. 2009. Rice endosperm iron biofortification by targeted and synergistic action of nicotianamine synthase andiferritin. Plant Biotechnol. J. 7:631-644.

Wolbang, C.M., D.P. Singh, S.R. Sykes, J.K. McInerney, A.R. Bird, and M.T. Treeby. 2010. Influence of pre- and postharvest factors on $\beta$-carotene content, its in vitro bioaccessibility, and antioxidant capacity in melons. J. Agric. Food Chem. 58:1732-1740.

Wolfenbarger, L.L., and P.R. Phifer. 2000. The ecological risks and benefits of genetically engineered plants. Science 290:2088-2093.

Wolson, R.A. 2007. Assessing the prospects for the adoption of biofortified crops in South Africa. AgBioForum 10:184-191.

Wong, J.C., R.J. Lambert, E.T. Wurtzel, and T.R. Rocheford. 2004. QTL and candidate genes phytoene synthase and $\zeta$-carotene desaturase associated with the accumulation of carotenoids in maize. Theor. Appl. Genet. 108:349-359.

Worzella, W.W., R. Khalidy, Y. Badawi, and S. Doghir. 1965. Inheritance of $\beta$-carotene in grain sorghum hybrids. Crop Sci, 5:591-592.

WSFS (World Summit on Food, Security), 2009. Declaration of the world summit on food security. http://www.fao.org/fileadmin/templates/wsfs/summit.

Wu, C.-Y, L.-L. Lu, X.-E. Yang, Y. Feng, Y.-Y. Wei, H.-L. Hao, P.J. Stoffella, and Z.-L. He. 2010. Uptake, translocation, and remobilization of zinc absorbed at different growth stages by rice genotypes of different Zn densities. J. Agric. Food Chem. 58:6767-6773.

Xie, W., and E. Nevo. 2008. Wild emmer: Genetic resources, gene mapping and potential for wheat improvement. Euphytica 164:603-614.

Xu, X.-H., H.-J. Zhao, Q.-L. Liu, T. Frank, K.-H. Engel, G. An, and Q.-Y. Shụ. 2009. Mutations of the multi-drug resistance-associated protein ABC transporter gene 5 result in reduction of phytic acid in rice seeds. Theor. Appl. Genet. 119:75-83.

Yan, J., C.B. Kandianis, C.E. Harjes, L. Bai, E.-H. Kim, X. Yang, D.J. Skinner; Z. Fu, S. Mitchell, Q. Li, M.G.S. Fernandez, M. Zaharieva, R. Babu, Y. Fu, N. Palacios, J. Li, D. DellaPenna, T. Brutnell, E.S. Buckler, M.L. Warburton, and T. Rocheford. 2010. Rare genetic variation at Zea mays crtRBI increases $\beta$-carotene in maize grain. Nat. Genet. 42:322-327.

Yang, Z., K.G. Dewey, B. Lönnerdal, O. Hernell, C. Chaparro, S. Adu-Afarwuah, E.D. McLean, R.J. Cohen, M. Domellöf, L.H. Allen, and K.H. Brown. 2008. Comparison of plasma ferritin concentration with the ratio of plasma transferring receptor to ferritin in estimating body iron stores: Results of 4 intervention trials. Am. J. Clin. Nutr. 87:1892-1898.

Yang, X., J. Yan, T. Shah, M.L. Warburton, Q. Li, L. Li, Y. Gao, Y. Chai, Z. Fu, Y. Zhou, S. Xu, G. Bai, Y. Meng, Y. Zheng, and J. Li. 2010. Genetic analysis and characterization of a new 
maize association mapping panel for quantitative trait loci dissection. Theor. Appl. Genet. 121:417-431.

Ye, X., S. Al-Babili, A. Klöti, J. Zhang, P. Lucca, P. Beyer, and I. Potrykus. 2000. Engineering. the provitamin A ( $\beta$-carotene) biosynthetic pathway into (carotenoid-freed) rice endosperm. Science 287:303-305.

Yeung, C.K. R.P. Glahn, X. Wu, R.H. Liu, and D.D. Miller. 2003. In vitro iron bioavailability and antioxidant activity of raisins. J. Food Sci. 68:701-705.

Yuan, F.J., H.J. Zhao, X.L. Ren, S.L. Zhu, X.J. Fu, and Q.Y. Shu. 2007. Generation and characterization of two novel low phytate mutations in soybean (Glycine max L. Merr.). Theor. Appl. Genet. 115:945-957.

Yun, S., J.-P. Habicht, D.D. Miller, and R.P. Glahn, 2004. An in vitro digestion/Caco-2 cell culture system accurately predicts the effects of ascorbic acid and polyphenolic compounds on iron bioavailability in humans. J. Nutr. 134:2717-2721.

Zapata-Caldas, E., G. Hyman, H. Pachón, F.A. Monserrate, and L.V. Varela. 2009. Identifying candidate sites for crop biofortification in Latin America: Case studies in Colombia, Nicaragua and Bolivia. Int. J. Health Geogr. 8:29.

Zee, S.Y. 1972. Vascular tissue and transfer cell distribution in the rice spikelet. Aust. J. Biol. Sci. 25:411-414.

Zee, S.Y., and T.P. O'Brian. 1970. A special type of tracheary element associated with 'xylem discontinuity' in the floral axis of wheat. Aust. J. Biol. Sci. 23:783-791.

Zhang, Y., Q. Song, J. Yan, J. Tang, R. Zhao, Y. Zhang, Z. He, C. Zou, and I. OrtizMonasterio. 2010. Mineral element concentrations in grains of Chinese wheat cultivars. Euphytica 174:303-313.

Zhao, H:-J., Q.-L. Liu, X.-L. Ren, D.-X. Wu, and Q.-Y. Shu. 2008. Gene identification and allele-specific marker development for two allelic low phytic acid mutations in rice (Oryza sativa L.). Mol. Breed. 22:603-612.

Zhao, F.J., Y.H. Su, S.J. Dunham, M. Rakszegi, Z. Bedo, S.P. McGrath, and P.R. Shewry. 2009. Variation in mineral micronutrient concentrations in grain of wheat lines of diverse origin. J. Cereal Sci. 49:290-295.

Zheng, L., Z. Cheng, C. Ai, X. Jiang, X. Bei, Y. Zheng, R.P. Glehn, R.M. Welch, D.D. Miller, X.G. Lei, and H. Shou. 2010. Nicotianamine, a novel enhancer of rice iron bioavailability to humans. PLoS One 5:e.10190.

Zhu, C., S. Naqvi, J. Breitenbach, G. Sandmann, P. Christou, and T. Capell. 2008. Combinatorial genetic transformation generates a library of metabolic phenotypes for the carotenoids pathway in maize. Proc. Natl. Acad. Sci. USA 105:18232-18237.

Zhu, C., S. Naqvi, S. Gómez-Galera, A.M. Pelacho, T. Capell, and P. Christou. 2007. Transgenic strategies for the nutritional enhancement of plants. Trends Plant Sci. 12:548-555.

Ziang, W., P.C. Struik, J. Lingna, H. van Keulen, Z. Ming, and T.J. Stomph. 2007. Uptake and distribution of root-applied or foliar-applied ${ }^{65} \mathrm{Zn}$ after flowering in aerobic rice. Ann. Appl. Biol. 150:383-391.

Ziang, W., P.C. Struik, H. van Keulen, M. Zhao, L.N. Jin, and T.J. Stomph, 2008. Does increased zinc uptake enhance grain zinc in rice? Ann. Appl, Biol, 153:135-147.

Zimmerman, M., and R. Hurrel. 2002. Improving iron, zinc and vitamin A nutrition through plant biotechnology. Curr. Opin. Biotechnol. 13:142-145. 